Florida International University FIU Digital Commons

FIU Electronic Theses and Dissertations

University Graduate School

7-13-2010

\title{
Spectroscopy of Electroproduced Light to Medium Mass Lambda Hypernuclei
}

Pavlo Baturin

Florida International University, pbatu001@fiu.edu

DOI: $10.25148 /$ etd.FI10081218

Follow this and additional works at: https://digitalcommons.fiu.edu/etd

\section{Recommended Citation}

Baturin, Pavlo, "Spectroscopy of Electroproduced Light to Medium Mass Lambda Hypernuclei" (2010). FIU Electronic Theses and Dissertations. 249.

https://digitalcommons.fiu.edu/etd/249

This work is brought to you for free and open access by the University Graduate School at FIU Digital Commons. It has been accepted for inclusion in FIU Electronic Theses and Dissertations by an authorized administrator of FIU Digital Commons. For more information, please contact dcc@fiu.edu. 


\section{FLORIDA INTERNATIONAL UNIVERSITY}

Miami, Florida

\section{SPECTROSCOPY OF ELECTROPRODUCED LIGHT TO MEDIUM MASS LAMBDA HYPERNUCLEI}

A dissertation submitted in partial fulfillment of the

requirements for the degree of

DOCTOR OF PHILOSOPHY

in

PHYSICS

by

Pavlo Baturin

2010 


\section{To: Dean Kenneth Furton}

College of Arts and Sciences

This dissertation, written by Pavlo Baturin, and entitled Spectroscopy of Electroproduced Light to Medium Mass Lambda Hypernuclei, having been approved in respect to style and intellectual content, is referred to you for judgment.

We have read this dissertation and recommend that it be approved.

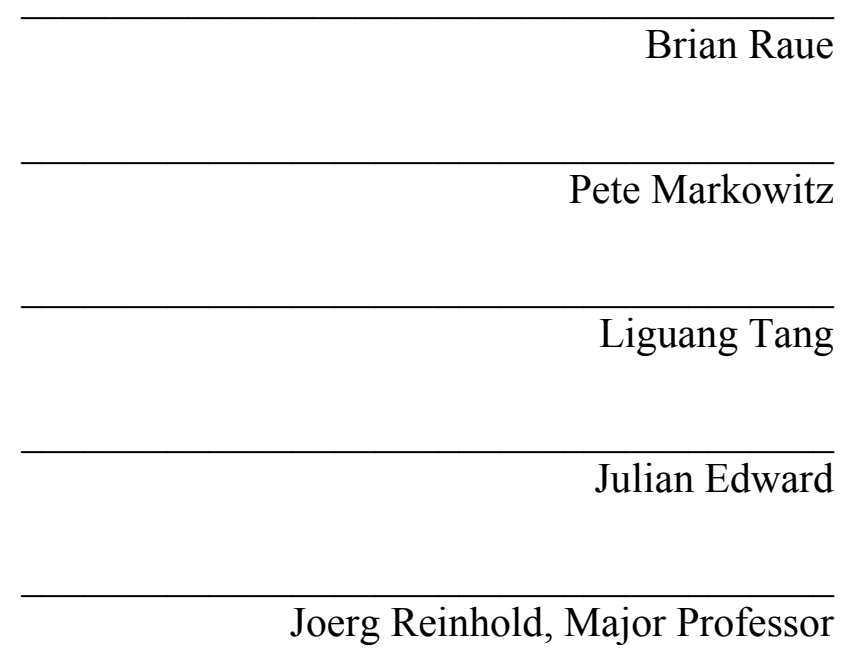

Date of Defense: July 13, 2010

The dissertation of Pavlo Baturin is approved.

Dean Kenneth Furton
College of Arts and Sciences

Interim Dean Kevin O'Shea University Graduate School

Florida International University, 2010 


\title{
ABSTRACT OF THE DISSERTATION \\ SPECTROSCOPY OF ELECTROPRODUCED LIGHT TO MEDIUM MASS \\ LAMBDA HYPERNUCLEI
}

\author{
by
}

Pavlo Baturin

Florida International University, 2010

Miami, Florida

\section{Professor Joerg Reinhold, Major Professor}

The E01-011 experiment at Jefferson Laboratory (JLab) studied light-to-medium mass $\Lambda$ hypernuclei via the ${ }^{A} Z+e \rightarrow{ }_{\Lambda}^{A}(Z-1)+e^{\prime}+K^{+}$electroproduction reaction. Precise measurement of hypernuclear ground state masses and excitation energies provides information about the nature of hyperon-nucleon interactions.

Until recently, hypernuclei were studied at accelerator facilities with intense $\pi^{+}$ and $K^{-}$meson beams. The poor quality of these beams limited the resolution of the hypernuclear excitation energy spectra to about $1.5 \mathrm{MeV}$ (FWHM). This resolution is not sufficient for resolving the rich structure observed in the excitation spectra. By using a high quality electron beam and employing a new high resolution spectrometer system, this study aims to improve the resolution to a few hundred keV with an absolute precision of about $100 \mathrm{keV}$ for excitation energies.

In this work the high-resolution excitation spectra of ${ }_{\Lambda}^{12} \mathrm{~B},{ }_{\Lambda}^{7} \mathrm{He}$, and ${ }_{\Lambda}^{28} \mathrm{Al}$ hypernuclei are presented. In an attempt to emphasize the presence of the core-excited states we introduced a novel likelihood approach to particle identification (PID) to serve 
as an alternative to the commonly used standard hard-cut PID. The new method resulted in almost identical missing mass spectra as obtained by the standard approach. An energy resolution of approximately 400-500 keV (FWHM) has been achieved, an unprecedented value in hypernuclear reaction spectroscopy. For ${ }_{\Lambda}^{12} \mathrm{~B}$ the core-excited configuration has been clearly observed with significant statistics. The embedded $\Lambda$ hyperon increases the excitation energies of the ${ }^{11} \mathrm{~B}$ nuclear core by $0.5-1 \mathrm{MeV}$. The ${ }_{\Lambda}^{7} \mathrm{He}$ spectrum has been observed with significant statistics for the first time. The ground state is bound deeper by roughly $400 \mathrm{keV}$ than currently predicted by theory. Indication for the core-excited doublet, which is unbound in the core itself, is observed. The measurement of ${ }_{\Lambda}^{28} \mathrm{Al}$ provides the first study of a $d$-shell hypernucleus with sub-MeV resolution. Discrepancies of up to $2 \mathrm{MeV}$ between measured and theoretically predicted binding energies are found. Similar disagreement exists when comparing to the ${ }_{\Lambda}^{28} \mathrm{Si}$ mirror hypernucleus. Also the core-excited structure observed between the major $s$-, $p$ - and $d$-shell $\Lambda$ orbits is not consistent with the available theoretical calculations.

In conclusion, the discrepancies found in this study will provide valuable input for the further development of theoretical models. 


\section{TABLE OF CONTENTS}

CHAPTER

PAGE

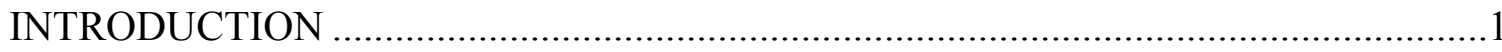

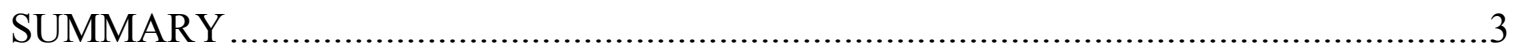

CHAPTER 1 THEORY OF HYPERNUCLEAR PHYSICS ……………………...........

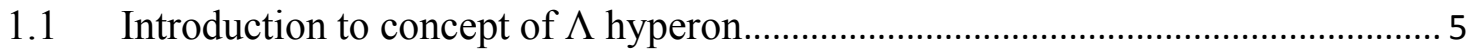

$1.2 \Lambda$ decay modes and lifetime...............................................................................

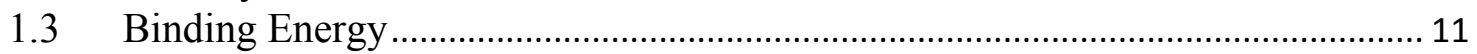

$1.4 \quad$ Nuclear Shell Model ......................................................................................... 16

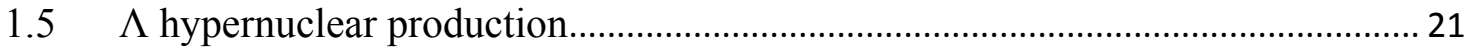

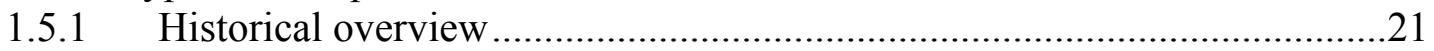

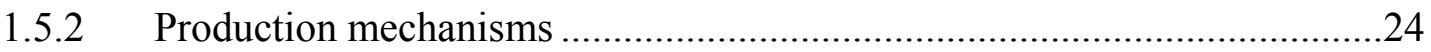

1.5.3 Kinematics of elementary electroproduction reaction ..................................32

1.5.4 Hypernuclear electroproduction process.....................................................35

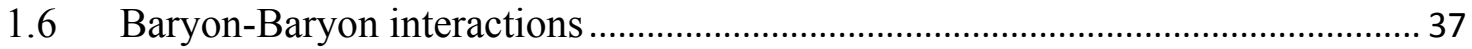

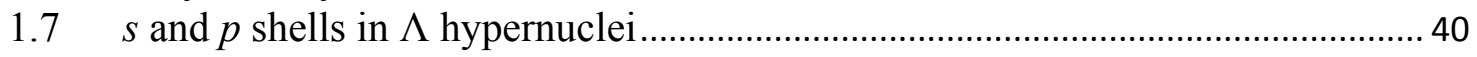

1.8 Significance of research and recent experimental data ....................................... 42

1.9 Dissertation Objectives .............................................................................. 45

CHAPTER 2 EXPERIMENTAL SETUP AND TECHNIQUES....................................47

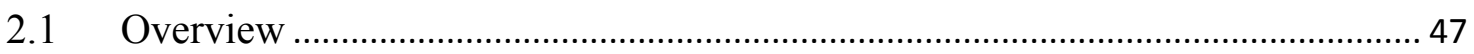

2.2 Experimental facility (Jefferson Laboratory) ……………………………….... 47

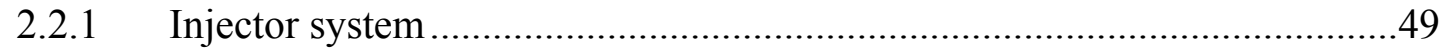

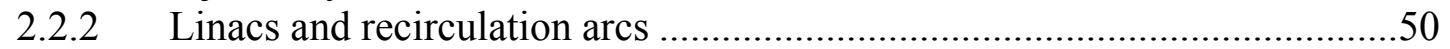

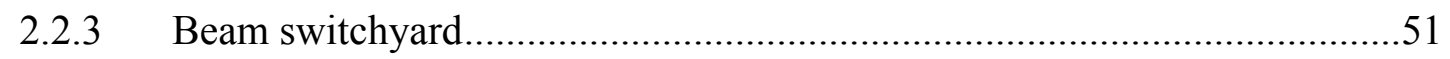

2.2.4 CEBAF beam properties ......................................................................

2.2.5 Hall $\mathrm{C}$ arc and beamline monitoring equipment .........................................52

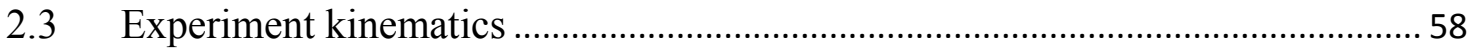

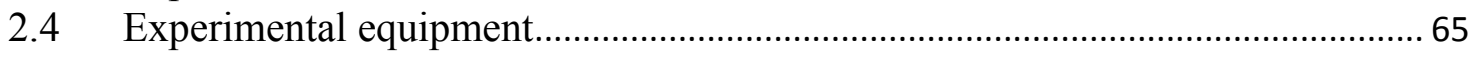

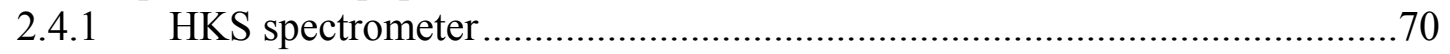

2.4.2 ENGE spectrometer ……………………….................................73

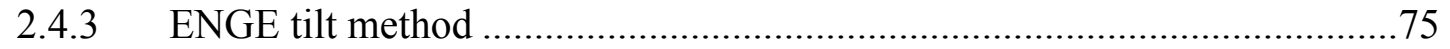

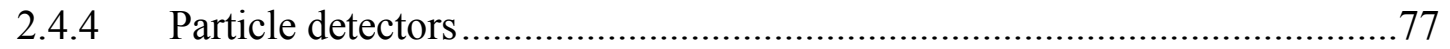

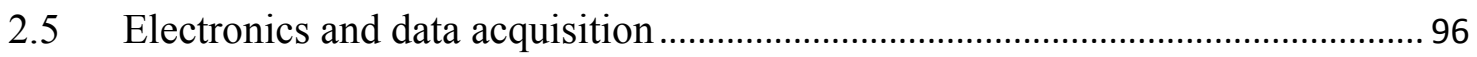

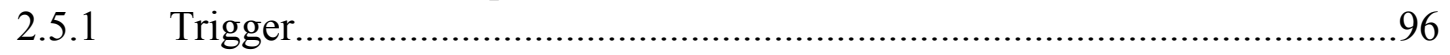

2.5.2 Read-out electronics...........................................................................102

2.5.3 Data acquisition system .........................................................................104

CHAPTER 3 AEROGEL CHERENKOV DETECTORS ………………....................106 
3.1 Cherenkov radiation principles...................................................................... 106

3.2 Experimental requirements and expectations .................................................... 109

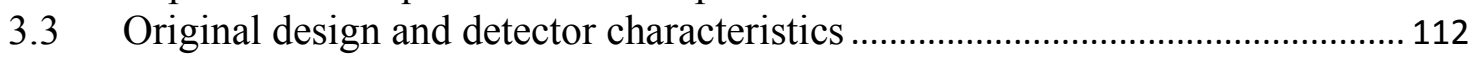

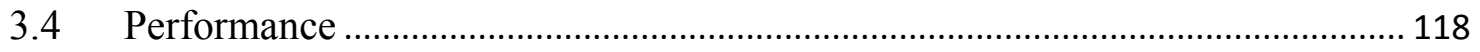

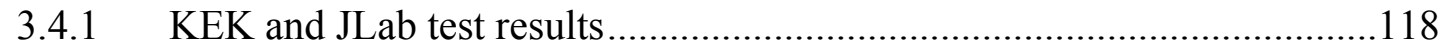

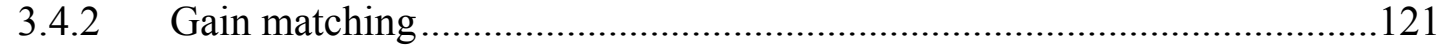

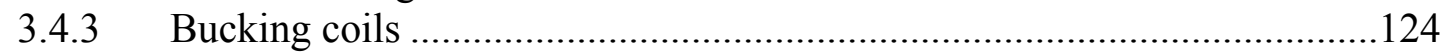

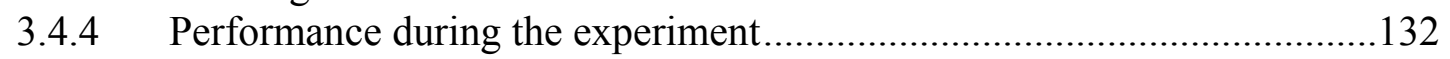

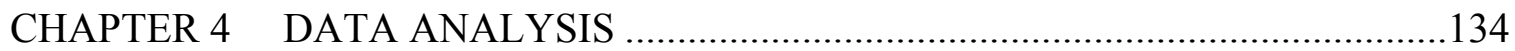

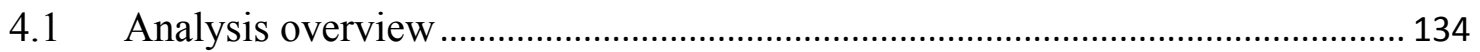

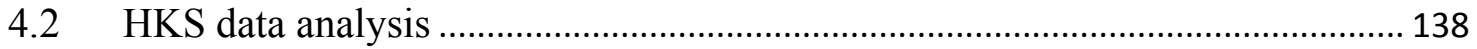

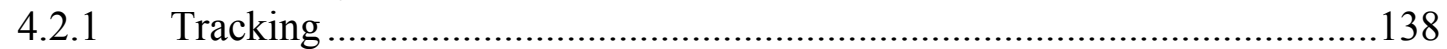

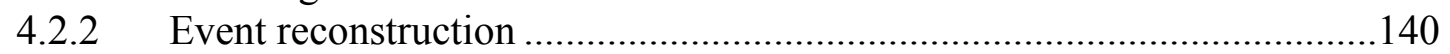

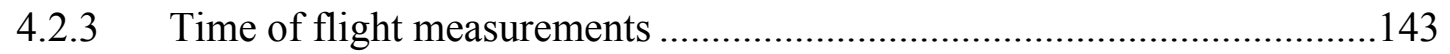

4.2.4 Particle Identification.........................................................................145

4.3 ENGE data analysis ……………………………..................................... 173

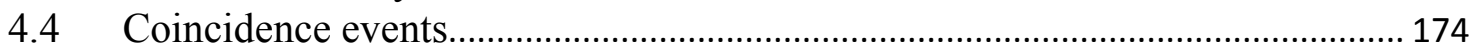

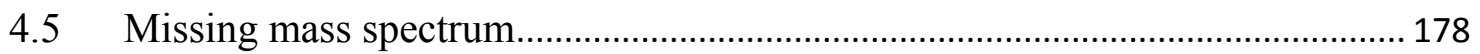

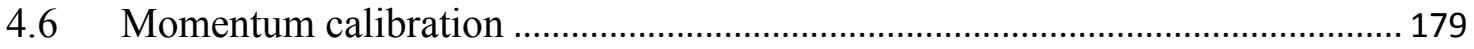

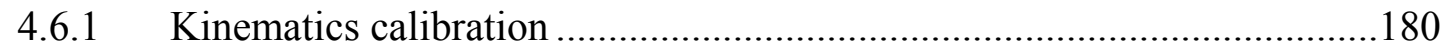

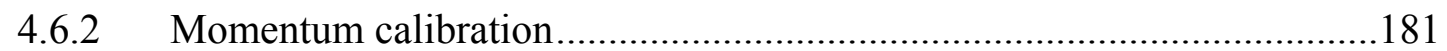

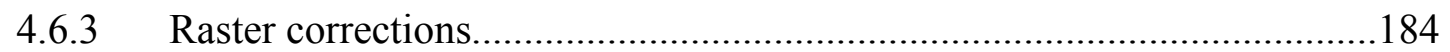

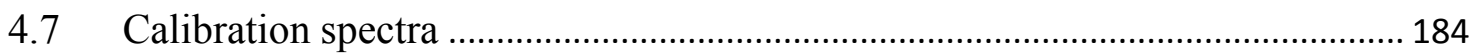

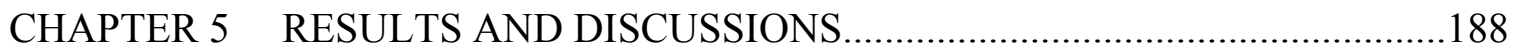

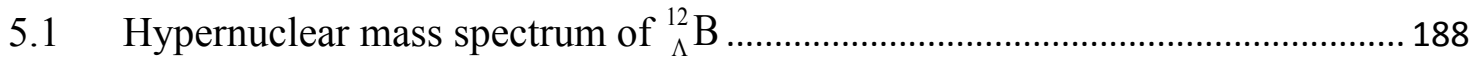

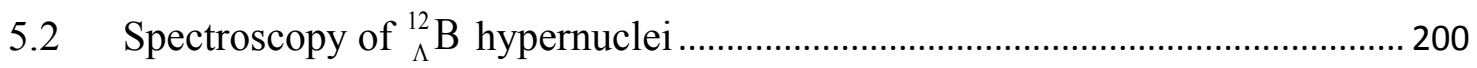

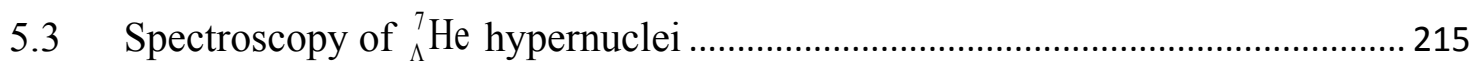

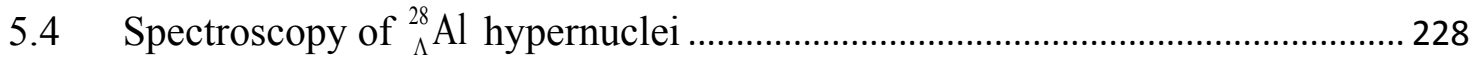

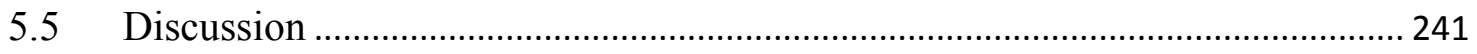

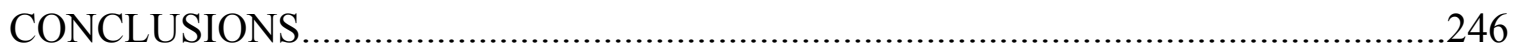

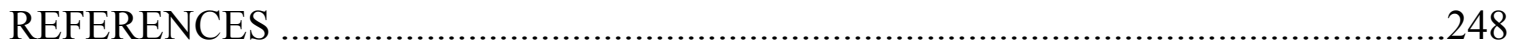

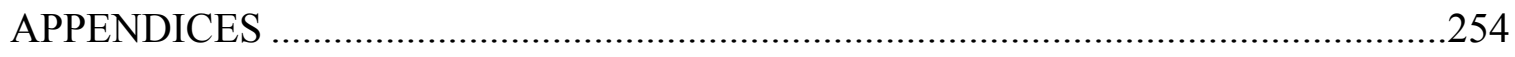

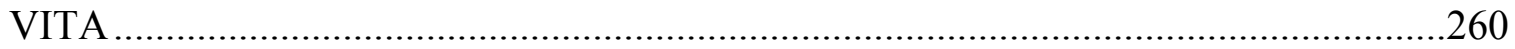




\section{LIST OF TABLES}

TABLE

PAGE

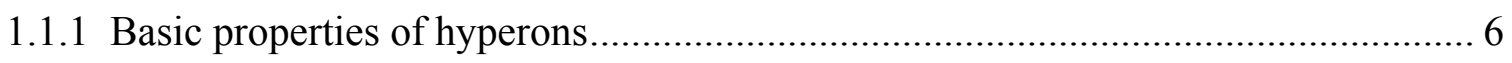

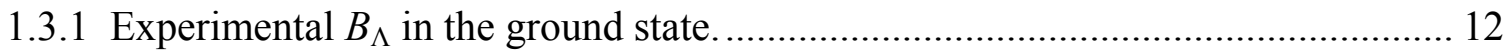

1.3.2 Experimental $B_{\Lambda}$ for heavy hypernuclei $(A \geq 12)$ in ground and excited states .... 14

1.4.1 Level distribution in the shells of the nucleus (Woods-Saxon potential with

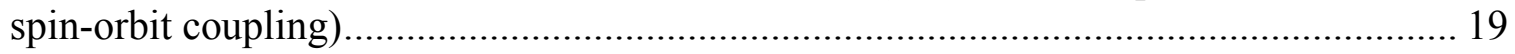

1.5.1 Properties of hypernuclear production reactions ................................................... 31

2.2.1 Principal CEBAF electron beam parameters .................................................. 52

2.2.2 Principal parameters of HMS and SOS ............................................................. 54

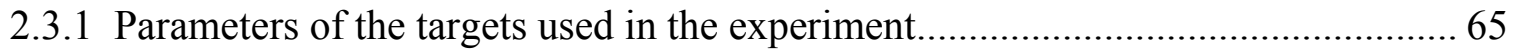

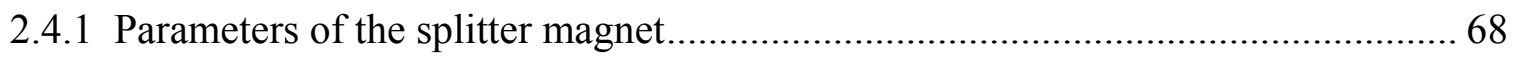

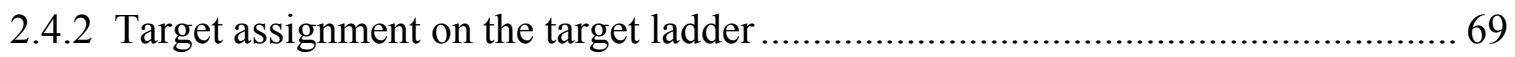

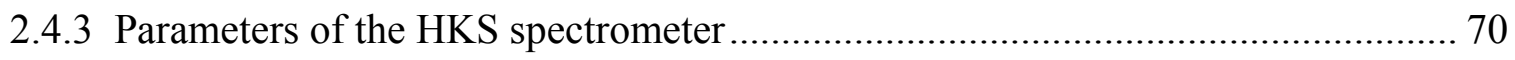

2.4.4 Specifications of the HKS magnets: Q1, Q2, and D ............................................ 71

2.4.5 Operational conditions of Q1, Q2, and D magnets during the run period .............. 72

2.4.6 Enge split-pole spectrometer parameters ......................................................... 74

2.4.7 ENGE honeycomb drift chamber (EDC) parameters ............................................ 80

2.4.8 Parameters of ENGE hodoscope system........................................................ 83

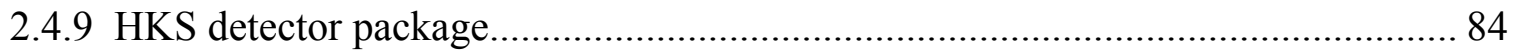

2.4.10 Geometrical and operational parameters of HKS drift chambers ......................... 89

2.4.11 Parameters of the HKS hodoscope system ....................................................... 91

2.4.12 Water Cherenkov detector parameters .......................................................... 96 
2.5.1 LeCroy 8LM input and output signals 102

2.5.2 Properties of F1 multihit TDC module (in normal and high resolution modes)... 102

2.5.3 Detector read-out electronics 103

3.2.1 Threshold momentum $p_{\text {th }}$ of particles of interest for select radiator refractive indexes......

3.3.1 Silica aerogel basic characteristics. 115

3.3.2 Geometrical properties of aerogel Cherenkov (AC) detectors 115

3.3.3 General characteristics of PMTs used in AC detectors 115

3.4.1 Results of AC counters performance tested in EEL clean room at JLab. 120

3.4.2 Gain matching for $\mathrm{AC} 1$ segment 1 (1T and $1 \mathrm{~B}$ tubes) 122

4.2.1 Geometrical parameters of the ENGE and HKS sieve slits 142

4.2.2 List of pdf variables per particle used in the likelihood PID analysis..... 151

4.2.3 WC parameterization for n.p.e. versus $\sin ^{2}(\theta)$ dependence 164

5.1.1 Comparison of ${ }_{\Lambda}^{12} \mathrm{~B}$ spectra created by likelihood and hard-cut PID techniques.. 198

5.2.1 Some of the possible ${ }_{\Lambda}^{12} \mathrm{~B}$ states from coupling by Equation (5.2.1). 201

5.2.2 Theoretical predictions of excitation energy $E_{x}$ of ${ }_{\Lambda}^{12} \mathrm{~B}$ hypernuclear states......... 203

5.2.3 Comparison of theoretical and experimental excitation energies for ${ }_{\Lambda}^{12} \mathrm{~B}$ spectrum. 207

5.2.4 Experimental energy resolution of ${ }_{\Lambda}^{12} \mathrm{~B}$ states 208

5.2.5 Comparison of the ${ }_{\Lambda}^{12} \mathrm{~B}$ spectroscopy obtained by E89-099 in Hall C, E94-107 in Hall A and E01-011 (current study) experiments. 210

5.2.6 Fitting results for ${ }_{\Lambda}^{12} \mathrm{C}$ spectra obtained in FINUDA at DAФNE and E369 at KEK 
5.2.7 Excitation energies of the first two excited states of carbon and boron

5.2.8 The change in excitation energy of $1 / 2^{-}$and $3 / 2^{-}$excited states......................... 215

5.3.1 Hypernuclear states of ${ }_{\Lambda}^{7} \mathrm{He}$ and their theoretically predicted binging energies and cross sections

5.3.2 Comparison of experimental results to theoretical predictions for ${ }_{\Lambda}^{7} \mathrm{He}$ hypernuclei 220

5.3.3 Fitting results for ${ }_{\Lambda}^{7} \mathrm{Li}$ spectrum measured at KEK by E336 experiment 225

5.3.4 Excitation energies of the $I=1$ ground and first excited states of lithium and helium ...... 226

5.3.5 The change in excitation energy of the first excited and ground states of lithium and helium. 227

5.4.1 Theoretical calculations of ${ }_{\Lambda}^{28} \mathrm{Al}$ energy spectrum with cross sections 229

5.4.2 Comparison of experimental results for ${ }_{\Lambda}^{28} \mathrm{Al}$ with theoretical predictions. 232

5.4.3 Fitting results for ${ }_{\Lambda}^{28} \mathrm{Al}$ spectrum 236

5.4.4 Fitting results for ${ }_{\Lambda}^{28} \mathrm{Si}$ spectrum obtained in AGS at BNL 240

5.4.5 Fitting results for ${ }_{\Lambda}^{28} \mathrm{Si}$ spectrum obtained in E369 at KEK 240

1.A Data summary for ${ }^{12} \mathrm{C}$ target $\left({ }_{\Lambda}^{12} \mathrm{~B}\right.$ hypernucleus $)$. 254

2.A Data summary for ${ }^{7} \mathrm{Li}$ target $\left({ }_{\Lambda}^{7} \mathrm{He}\right.$ hypernucleus $)$ 255

3.A Data summary for ${ }^{28} \mathrm{Si}$ target $\left({ }_{\Lambda}^{28} \mathrm{Al}\right.$ hypernucleus $)$ 255

4.A List of parameter files used in likelihood PID routine. 256

5.A List of the flags used in likelihood PID routine. 256

6.A Summary of flags used for creating ntuple for likelihood parameterization 257 
1.B Summary of the hard cuts used in standard approach to PID

2.B Cuts used in likelihood PID analysis.. 


\section{LIST OF FIGURES}

FIGURE

PAGE

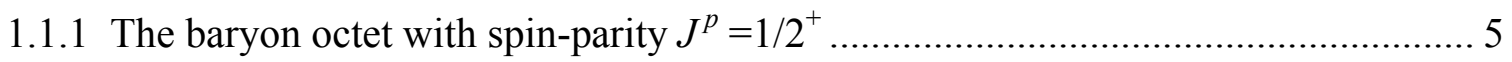

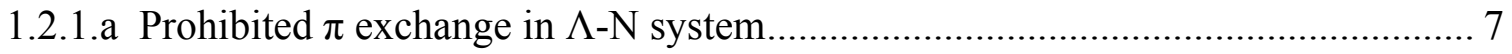

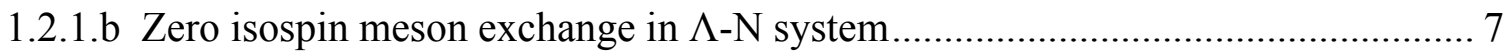

1.2.1.c Strange meson exchange in $\Lambda-\mathrm{N}$ system................................................... 7

1.2.2.a Blocked mesonic decay of $\Lambda$ with low energy of released nucleon .................... 8

1.2.2.b Non-mesonic decay of $\Lambda$ with high energetic recoiled nucleons ....................... 8

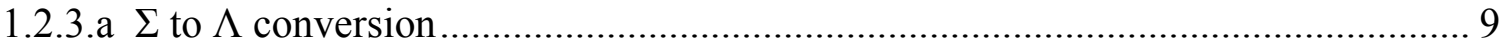

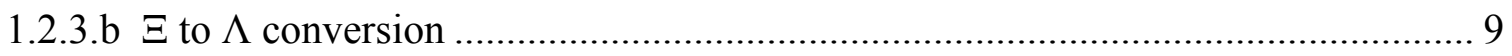

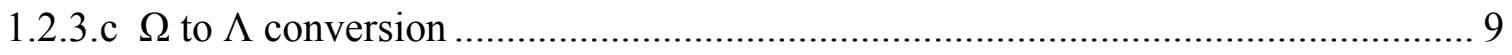

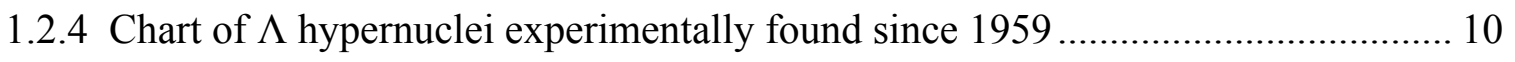

1.3.1 $\Lambda$ binding energy as a function of atomic number (data from Table 1.3.1) .......... 13

1.3.2 $\Lambda$ binding energy as a function of atomic number..................................... 15

1.4.1 Single-particle energy levels in the nuclear central potential (Woods-Saxon)...... 20

1.5.1 Emulsion photo of the first hypernuclear event ........................................... 21

1.5.2 Quark flow schematic diagrams of $\Lambda$ hypernuclear production .......................... 25

1.5.3 Hyperon recoil momentum in various elementary reactions at $\theta=0^{\circ} \ldots \ldots \ldots \ldots \ldots \ldots . . .28$

1.5.4 Kinematics of the hypernuclear electroproduction .......................................... 32

1.5.5 Schematic representation of ${ }^{A} X\left(e, e^{\prime} K^{+}\right){ }_{\Lambda}^{A} Z$ hypernuclear electroproduction...... 35

1.8.1 Hypernuclear binding energy spectrum of ${ }_{\Lambda}^{12} \mathrm{C}$ from BNL (left) and KEK (right) produced by $\left(\pi^{+}, K^{+}\right)$reaction............................................................... 43 
1.8.2 The HNSS hypernuclear spectrum of ${ }_{\Lambda}^{12} \mathrm{~B}$

2.2.1 Schematic top view of CEBAF accelerator ......................................................... 48

2.2.2 Aerial view of the CEBAF accelerator complex ................................................... 49

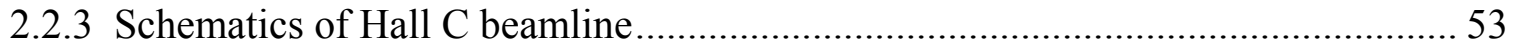

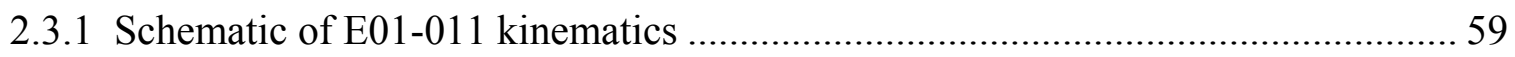

2.3.2 Total cross section of $\Lambda$ photoproduction reaction $p\left(\gamma, K^{+}\right) \Lambda \ldots \ldots \ldots \ldots \ldots \ldots \ldots \ldots \ldots . . . .61$

2.3.3 Kaon angular distribution of ${ }_{\Lambda}^{12} \mathrm{~B}$ ground state doublet cross section for

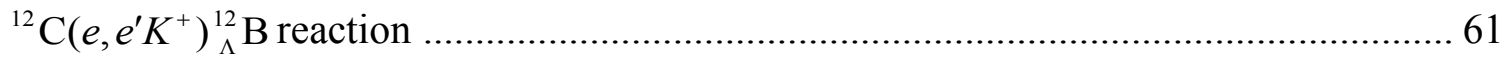

2.3.4 Angular distribution of the virtual photon flux and bremsstrahlung scattering ..... 63

2.4.1 The schematics of the E01-011 experimental setup ..............................................6 65

2.4.2 Splitter magnet. Schematic design on the left and the actual photo on the right.... 67

2.4.3 Schematic, "TOP view" of the splitter magnet and target chamber. ...................... 67

2.4.4 Target ladder inside of the chamber with empty target holes on the left and with

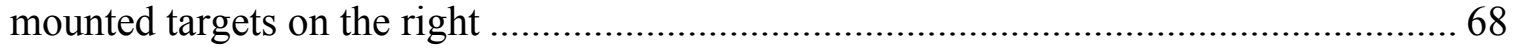

2.4.5 Photo of the HKS spectrometer inside of the Hall C .............................................. 71

2.4.6 Schematic representation of the components of QQD HKS spectrometer ............. 72

2.4.7 Sketch of Enge split-pole spectrometer (top view) ............................................... 74

2.4.8 Magnetic field versus current $(B-I)$ curve obtained at the JLab test facility........... 75

2.4.9 Schematic view of the ENGE tilt method.......................................................... 75

2.4.10 Tilted ENGE spectrometer............................................................................. 76

2.4.11 Left: Electron rate dependence on the ENGE tilt angle...................................... 76

2.4.12 Enge detector package ……………............................................................... 78

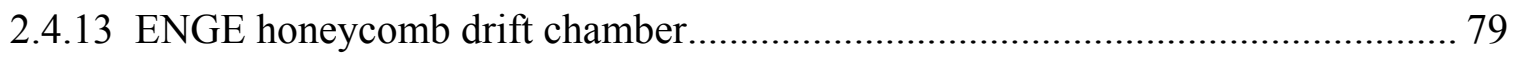


2.4.14 ENGE honeycomb drift chamber in EEL building at Jefferson Lab . 81

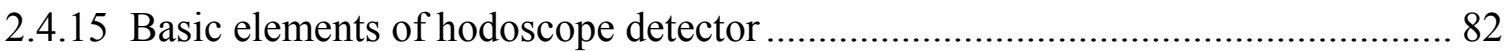

2.4.16 ENGE hodoscope system (beam's eye) ...................................................... 83

2.4.17 Schematic layout of HKS detector package................................................. 85

2.4.18 Trajectories of the particles with different momenta....................................... 85

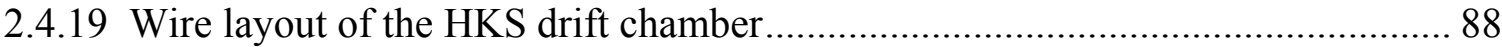

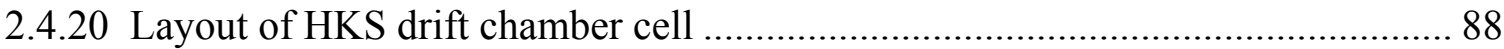

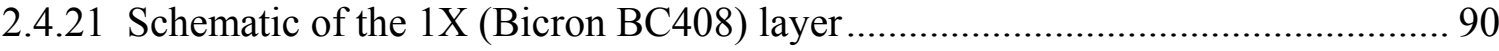

2.4.22 Schematic of the 1Y (Bicron BC408) layer.................................................... 91

2.4.23 Results from KEK test of water Cherenkov detectors with pure water and chemical water radiator

2.4.24 Schematic drawing of the water Cherenkov counters.................................... 95

2.4.25 Photograph of the water Cherenkov counters mounted on the HKS detector

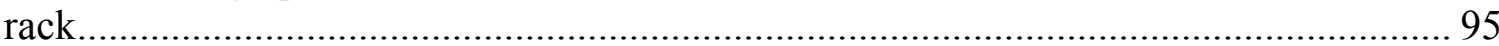

2.5.1 Particles with different momentum focus in different points ............................. 98

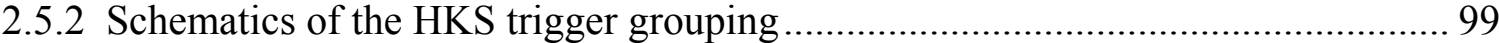

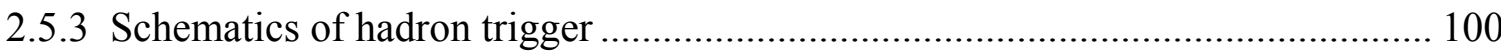

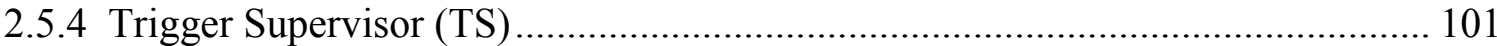

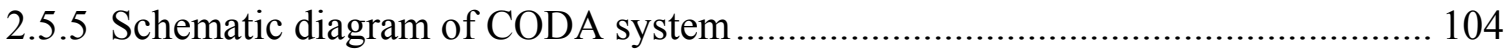

3.1.1.a Cherenkov wave propagation with coherent wavefront with a satisfied threshold contrition. 106

3.1.1.b Distribution of non-coherent waves in case of an unfulfilled threshold condition

3.2.1 Cherenkov detector n.p.e. as a function of particle momentum ( $p$ ) for three values of refractive index of radiator $(n)$

3.3.1 Schematics of the three aerogel Cherenkov counters (AC1, AC2, AC3) 114 
3.3.2 Dependence of SP-50 aerogel transmittance on the wavelength 115

3.3.3 Interior of the diffusion box (view from the PMT hole).

3.4.1 AC counter under test in EEL (JLab)

3.4.2 Example of the single photoelectron (p.e.) peak and cosmic ADC peak during testing at JLab clean room

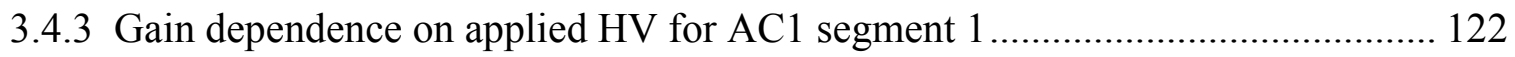

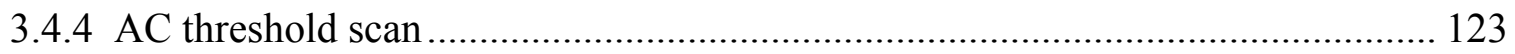

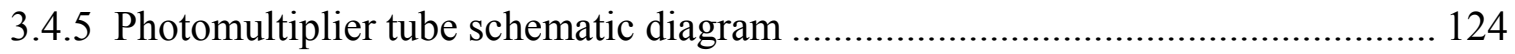

3.4.6 Schematics of the HKS setup and relative direction of fringe field lines ............ 125

3.4.7 Electron trajectories within PMT under influence of fringe field...................... 126

3.4.8 Effect of the fringe magnetic field on aerogel Cherenkov ADC signal............... 127

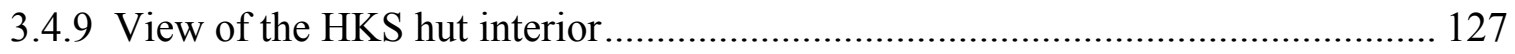

3.4.10 Reduction of the n.p.e. with increase of the current in the HKS dipole ............. 128

3.4.11 Improvement of n.p.e. in the presence of the iron bunker .............................. 128

3.4.12 Bucking coils with compensating local magnetic field ................................ 129

3.4.13 Photo of AC PMTs with bucking coils installed ......................................... 130

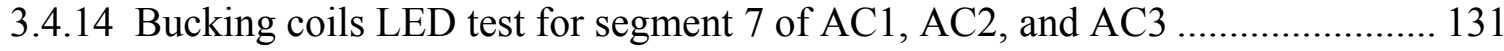

3.4.15 Number of AC photoelectrons as a function of run number............................ 133

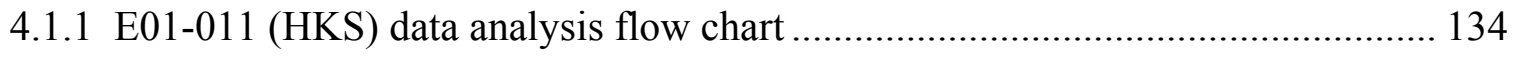

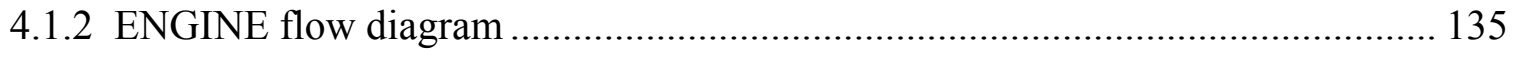

4.2.1 ENGE and HKS sieve slit collimator plates ............................................... 142

4.2.2 Time-walk effect due to a size variation of the signal ................................... 144

4.2.3 Schematics of the particles location in typical AC n.p.e. spectrum.................... 146 
4.2.4 Schematics of the particles location in typical single layer WC n.p.e.

spectrum.

4.2.5 Schematics of the particles location in the beta spectrum 148

4.2.6 Schematic diagram of likelihood PID analysis

4.2.7 Example of the $\mathrm{AC} 1$ segment 3 clean proton, kaon, and pion n.p.e.

distributions after applying hard PID cuts ....

4.2.8 The normalized AC1_seg3 probability density function (pdf) used in the likelihood PID analysis.....

4.2.9 Possible particle's trajectory through two neighboring segments in AC detector. 156

4.2.10 Projection of the particle track from focal plane on AC layer. 157

4.2.11 Geometrical overlap of the AC segments specified in the analysis software.... 157

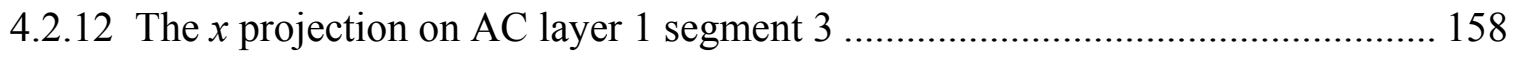

4.2.13 Example of the two neighbor segment fired at the same time. 160

4.2.14 Schematics of the $x_{A C_{L}}$ projections on segments 1,2,3, and etc. 160

4.2.15 $\mathrm{WC}_{1}$ and $\mathrm{WC}_{2}$ proton, kaon and pion spectra for $\pm 3 \%$ momentum cut around $1.25 \mathrm{GeV} / \mathrm{c}$ 161

4.2.16 The $\mathrm{WC}_{1}$ and $\mathrm{WC}_{2}$ normalized pdf distributions for protons, kaons and pions. 162

4.2.17 $\mathrm{WC}_{1}$ and $\mathrm{WC}_{2}$ n.p.e. dependence on squared sine of Cherenkov radiation angle. 164

4.2.18 Relative beta clean distributions 165

4.2.19 Normalized clean relative beta distributions on beta scale 166

4.2.20 TripleVoigt fit of the beta spectrum, with protons described by LeftVoigt, kaons - by CenralVoigt and pions - by RightVoigt functions.. 167

4.2.21 Example of AC, WC, and beta spectra obtained by likelihood PID. 170

4.2.22 The spectrum of relative kaon beta, $\beta$ - $\beta_{K}$, for standard hard-cut PID on the left and likelihood on the right...... 
4.2.23 Comparison of the kaons recovered by: a) Likelihood.AND.NOT.(Standard) kaon PID; b) Standard.AND.NOT.(Likelihood) kaon PID ........................................... 172

4.4.1 Coincidence time after all corrections applied.................................................. 176

4.4.2 Two dimensional profile of $\beta_{T O F}-\beta_{K}$ versus coincidence time …....................... 177

4.4.3 Coincidence time spectrum with true and accidental peaks separation

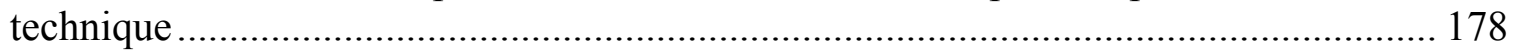

4.6.1 Flowchart of iteration process of optics calibration procedure............................ 179

4.6.2 Momentum correlation between recoil electron and kaon................................... 182

4.6.3 Missing mass spectra before (left) and after (right) matrix optimization ............. 183

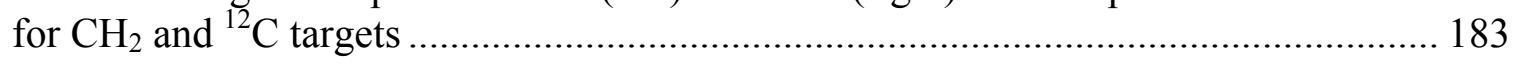

4.7.1 $\Lambda$ and $\Sigma^{0}$ peaks on missing mass spectrum of $\mathrm{CH}_{2}$ target................................ 185

4.7.2 Binding energy $\left(-B_{\Lambda}\right)$ spectrum of ${ }_{\Lambda}^{12} \mathrm{~B}$ with $150 \mathrm{keV} / \mathrm{bin}$ scale ........................ 186

4.7.3 Missing mass spectrum on $\mathrm{CH} 2$ target in HNSS experiment ............................. 187

5.1.1 Binding energy spectrum $\left(-B_{\Lambda}\right)$ of ${ }_{\Lambda}^{12} \mathrm{~B}$ hypernucleus obtained by likelihood

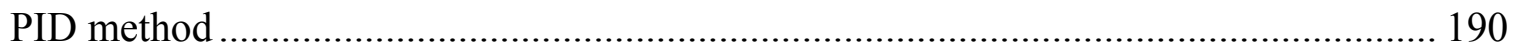

5.1.2 Binding energy spectrum $\left(-B_{\Lambda}\right)$ of ${ }_{\Lambda}^{12} \mathrm{~B}$ hypernucleus obtained by standard

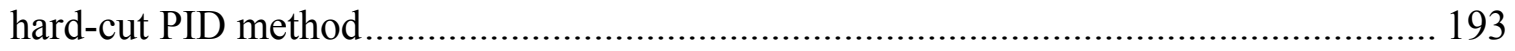

5.1.3 The comparison of signal to noise ratio for hypernuclear states obtained by hard-cut (blue diamonds) and likelihood (red circles) PID techniques

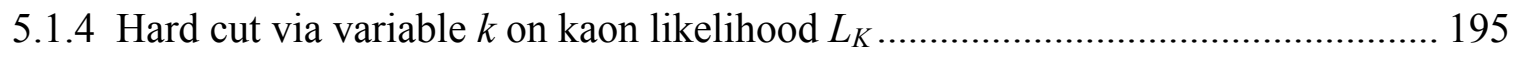

5.1.5 Likelihood correlations ................................................................................... 196

5.1.6 Scan of the ${ }_{\Lambda}^{12} \mathrm{~B}$ spectra for different coefficient $k$ in likelihood PID condition... 197

5.1.7 True coincident events versus coefficient $\mathrm{k}$ for peaks $1,2,3$, and 4 ..... 198

5.1.8 Side-by-side comparison of ${ }_{\Lambda}^{12} \mathrm{~B}$ spectra found by likelihood and hard-cut PID methods 
5.2.1 Schematics of the energy levels of ${ }^{11} \mathrm{~B}$ nuclear host and $\Lambda$ particle

5.2.2 Fit results for ${ }_{\Lambda}^{12} \mathrm{~B}$ binding energy $\left(-B_{\Lambda}\right)$ spectrum obtained by the likelihood PID method

5.2.3 The ${ }_{\Lambda}^{12} \mathrm{~B}$ excitation energy spectrum obtained in E94-107 experiment in Hall A at JLab

5.2.4 Visual comparison of the experimental excitation energy from its theoretically predicted value

5.2.5 The ${ }_{\Lambda}^{12} \mathrm{C}$ spectroscopy obtained in E369 experiment at KEK via $\left(\pi^{+}, K^{+}\right)$reaction (left) and in FINUDA experiment at DAФNE via $\left(K^{-}, \pi^{-}\right)$reaction (right).

5.2.6 The change in excitation energy of the $1 / 2^{-}$and $3 / 2^{-}$excited states when embedding a $\Lambda$ into the two mirror boron and carbon core nuclei

5.3.1 Binding energy spectrum $\left(-B_{\Lambda}\right)$ of ${ }_{\Lambda}^{7} \mathrm{He}$ hypernucleus (without core-excited peak)

5.3.2 Binding energy spectrum $\left(-B_{\Lambda}\right)$ of ${ }_{\Lambda}^{7} \mathrm{He}$ hypernucleus (with core-excited peak).

5.3.3 Level diagrams for ${ }^{6} \mathrm{He}$ and ${ }_{\Lambda}^{7} \mathrm{He}$ calculated according to four-body cluster model (left). 222

5.3.4 Results for $A=7$ iso-triplet from emulsion $\pi^{-}$decay experiments

5.3.5 Hypernuclear spectrum of ${ }_{\Lambda}^{7} \mathrm{Li}$ measured by SKS at KEK in E336 experiment.

5.3.6 The change in excitation energy of the first excited and ground states of lithium and helium based on experiments E336 (KEK) and E01-011 (HKS)

5.4.1 Binding energy $\left(-B_{\Lambda}\right)$ prediction for ${ }_{\Lambda}^{28} \mathrm{Al}$ hypernuclei 230

5.4.2 Divided contributions to the particle hole $J$-multiplet state for ${ }^{28} \operatorname{Si}\left(e, e^{\prime} K^{+}\right){ }_{\Lambda}^{28} \mathrm{Al}$ reaction at $E_{\gamma}=1.3 \mathrm{GeV}$ and $\theta_{K^{+}}^{L A B}=3^{\circ}$ with each pillar corresponding to differential cross section (SLA). 
5.4.3 Simulated binding energy $\left(-B_{\Lambda}\right)$ spectrum of ${ }_{\Lambda}^{28} \mathrm{Al}$ hypernuclei to be observed

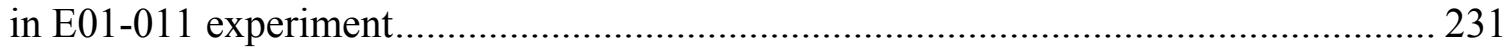

5.4.4 Experimental binding energy spectrum $\left(-B_{\Lambda}\right)$ of ${ }_{\Lambda}^{28} \mathrm{Al}$ hypernucleus .................... 233

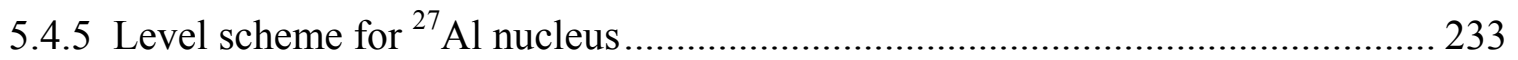

5.4.6 Predicted hypernuclear spectrum of ${ }_{\Lambda}^{28} \mathrm{Al}$ with marked core-excited states

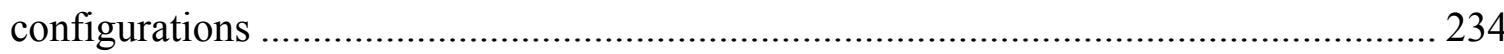

5.4.7 Binding energy spectrum $\left(-B_{\Lambda}\right)$ of ${ }_{\Lambda}^{28} \mathrm{Al}$ hypernucleus with core-excited states .... 235

5.4.8 Experimental (top) and theoretical (bottom) excitation energy spectra of ${ }_{\Lambda}^{28} \mathrm{Si}$

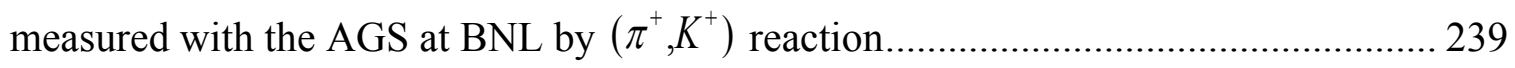

5.4.9 Excitation energy spectrum of ${ }_{\Lambda}^{28} \mathrm{Si}$ measured with the SKS spectrometer at

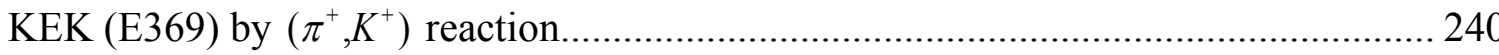




\section{INTRODUCTION}

One of the main tasks of nuclear physics is the study of subatomic particles and their interactions. Nowadays, the fundamental theory of strong interactions is a particularly interesting subject in the field. At the current moment, such a theory is not complete yet. It describes very well the nucleon-nucleon $(\mathrm{NN})$ interactions, which were intensively studied over the last several decades. In our modern, technically advanced world the research gravitates towards the higher energies, reaching deeper inside of the nuclear structure. About sixty years ago the strong interaction was associated with the interaction between nucleons responsible for holding those nucleons together within the nuclear volume. However, with discovery of mesons and strange particles, the picture has changed. The proof of bound states of strange baryons ( $\Lambda$ particles) with nucleons revealed a broad class of particles participating in the strong interaction, called hadrons. The rich variety of hadron interactions raises an important topic in modern nuclear physics which strives for providing a deep insight into nuclear matter structure. The analysis of the interaction of a strange baryon, called a hyperon, with a nucleon delivers new knowledge of nuclear properties, which were not understood with widely studied nucleon-nucleon interactions.

The direct approach for creating an interaction of free hyperons with nucleons in the target is not an easy task in experimental nuclear physics. The relatively short lifetime of free hyperons, which can only be produced as a secondary beam, leads to extremely low statistics. Nowadays, the best known method of hyperon-nucleon interaction study is the formation of hyperons inside of the nucleus. The bound hyperon serves as a probe of 
nuclear properties of such complex nuclear systems called hypernuclei. Hypernuclear physics itself is a sub-area of nuclear physics, which studies such bound systems. It employs the rich knowledge of the nucleon-nucleon interaction and at the same time performs a generalization of the above mentioned interaction for systems with a third quark flavor - strangeness [1]. Production reactions of $\Lambda$ particles and hypernuclei, as well as spectroscopy and decay modes, provide valuable information on the hyperon interaction. For example, analysis of $\Lambda$ and hypernuclear decay modes gives knowledge of the properties of weak interactions. The study of the energy of ground and excited states exposes the laws of baryon distribution inside of the nucleus. Investigation of $\Lambda \mathrm{N}$ and $\Lambda \Lambda$ potentials is important for baryon-baryon theories that include strange quarks, e.g. $\mathrm{SU}(3)$. These potentials are more short-ranged than the ones for $\mathrm{NN}$ and therefore the additional degrees of freedom play an essential role. 


\section{SUMMARY}

The dissertation is composed of the five chapters that include: 1) theoretical background of hypernuclear physics and reaction spectroscopy; 2) description of the experimental equipment; 3 ) technical details of the aerogel Cherenkov detectors and their performance during the experiment; 4) data analysis techniques; 5) discussion of the hypernuclear spectroscopy of ${ }_{\Lambda}^{12} \mathrm{~B},{ }_{\Lambda}^{7} \mathrm{He}$, and ${ }_{\Lambda}^{28} \mathrm{Al}$ with an emphasis on core-excited state configurations.

In Chapter 1 I provide a basic theoretical framework of hypernuclear physics and discuss the current situation in hypernuclear spectroscopy. I explain the importance of the electroproduction reaction that uniquely allows obtaining high precision spectra of exotic, neutron rich hypernuclei and medium to heavy mass number hypernuclei, which have never been studied before.

Chapter 2 explains the equipment and kinematics setup that have been used in the experiment. The details on the newly introduced tilt method are discussed. The chapter presents a description of both electron and kaon spectrometers and provides a thorough explanation of the working principles and technical specifications of particle detector packages for each arm.

In Chapter 3 I concentrate my attention on the aerogel Cherenkov detectors that have been constructed by me at the Jefferson Laboratory. The working principles of the detector are discussed and the technical parameters are presented in the tables. The results of the preliminary tests conducted in Jefferson Lab before the start of experiment are shown as well. The detectors' later performance during the experiment is reported. The 
technical difficulties that arose because of the presence of a fringe magnetic field from the high resolution kaon spectrometer (HKS) and the solution with the bucking coils wrapped around the photomultiplier tubes (PMT) are described in detail.

Chapter 4 goes step by step through each part of the data analysis. It describes the techniques used for detectors' calibration and parameterization. It explains in detail the methods applied in the current analysis and also gives a description of the methods commonly used in hypernuclear physics. The theoretical framework of the newly developed method of a likelihood approach to particle identification (PID) is presented. The results of its application in this analysis and the comparison with the standard hard cut PID are given.

By Chapter 5 I conclude the dissertation. In the final chapter I present the high resolution spectra for ${ }_{\Lambda}^{12} \mathrm{~B},{ }_{\Lambda}^{7} \mathrm{He}$, and ${ }_{\Lambda}^{28} \mathrm{Al}$. The spectroscopy of ${ }_{\Lambda}^{12} \mathrm{~B}$ is obtained by the likelihood PID method and quantitative analysis is performed. It also presents the comparison with spectroscopy results obtained by standard hard-cut PID approach. The likelihood PID method yields spectra of similar quality but not better than the standard approach. Because of that, the spectra for ${ }_{\Lambda}^{7} \mathrm{He}$ and ${ }_{\Lambda}^{28} \mathrm{Al}$, are found with the application of the standard hard-cut PID technique. The analysis includes the description of the coreexcited state configurations for each of the mentioned hypernuclei. The explanation of the results and detailed discussions are made. 


\section{CHAPTER 1}

\section{THEORY OF HYPERNUCLEAR PHYSICS}

\subsection{Introduction to concept of $\Lambda$ hyperon}

A hypernucleus is a complex nuclear system containing at least one bound hyperon strongly interacting with the nucleons inside the nucleus. It is formed by replacing at least one of the nucleons by a hyperon, for example $\Lambda$ (lambda), $\Sigma$ (sigma), $\Xi$ (cascade), $\Omega$ (omega). The $\Lambda$ is the lightest hyperon with strangeness quantum number $S=-1$. The mass of the $\Lambda\left(m_{\Lambda}=1115.683 \mathrm{GeV} / \mathrm{c}^{2}\right)$ is $20 \%$ higher compared to the nucleon. It has isospin $I=0$ and zero electrical charge. Together with the $\Sigma^{0}$, it is at the center of the baryon octet with spin-parity $J^{p}=1 / 2^{+}$(see Fig. 1.1.1).

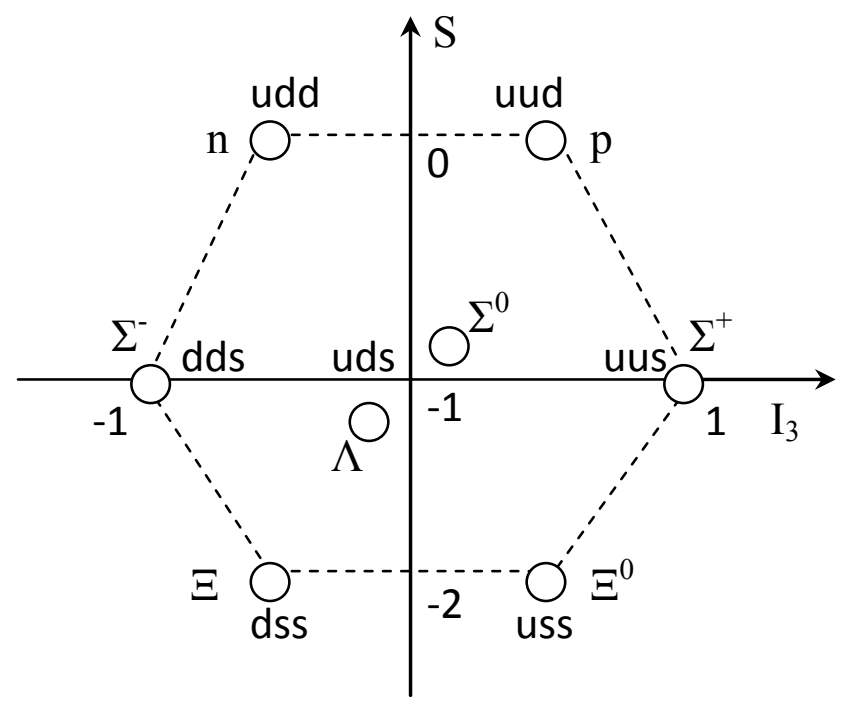

Figure 1.1.1 The baryon octet with spin-parity $J^{p}=1 / 2^{+}$. 
The $\Lambda$ is composed of one up $(\mathrm{u})$, one down $(\mathrm{d})$, and one strange (s) quarks. It shares the same quark assignment and electric charge with the $\Sigma^{0}$ hyperon. Only the value of isospin, the lifetime, and the mass difference sets them apart. Some of the basic properties of the $\Lambda$ and other strange particles are presented in Table 1.1.1.

The hypernucleus is designated by the symbol ${ }_{\mathrm{Y}}^{\mathrm{A}} Z$, where $Z$ is the symbol of the chemical element, $A$ is the total number of baryons (i.e., nucleons and hyperons inside of the nucleus), and $Y$ is the symbol of the corresponding hyperon. For example ${ }_{\Lambda}^{4} \mathrm{H}$ represents a hydrogen hypernucleus with three nucleons (one proton, two neutrons) and one bound $\Lambda$ hyperon.

Table 1.1.1 Basic properties of hyperons.

\begin{tabular}{cccccccc}
\hline Hyperon & Charge & Strangeness & Spin-parity & Isospin & Mass, $(\mathrm{MeV})$ & Lifetime, (s) & Decay \\
\hline$\Lambda$ & 0 & -1 & $1 / 2^{+}$ & 0 & 1115.7 & $2.6 \cdot 10^{-10}$ & $\mathrm{~N} \pi$ \\
$\Sigma^{+}$ & 1 & -1 & $1 / 2^{+}$ & 1 & 1189.4 & $0.8 \cdot 10^{-10}$ & $\mathrm{~N} \pi$ \\
$\Sigma^{0}$ & 0 & -1 & $1 / 2^{+}$ & 1 & 1192.6 & $7.4 \cdot 10^{-20}$ & $\Lambda \gamma$ \\
$\Sigma^{-}$ & -1 & -1 & $1 / 2^{+}$ & 1 & 1197.4 & $1.5 \cdot 10^{-10}$ & $\mathrm{~N} \pi$ \\
$\Xi^{0}$ & 0 & -2 & $1 / 2^{+}$ & $1 / 2$ & 1314.8 & $2.9 \cdot 10^{-10}$ & $\Lambda \pi$ \\
$\Xi^{-}$ & -1 & -2 & $1 / 2^{+}$ & $1 / 2$ & 1321.3 & $1.6 \cdot 10^{-10}$ & $\Lambda \pi$ \\
$\Omega^{-}$ & -1 & -3 & $3 / 2^{+}$ & 0 & 1672.5 & $0.8 \cdot 10^{-10}$ & $\Lambda K$ \\
\hline
\end{tabular}

Inside of the nucleus the nucleons occupy discrete energy levels with distinct angular momenta. The interaction between the nucleons can cause them to swap their places in the energy level spectrum. Because the total energy of the nucleus does not change, such energy level swaps are not observable [1]. With insertion of the $\Lambda$ hyperon inside of the nucleus as a probe, one can investigate the energy levels of an individual baryon. According to the Pauli Exclusion Principle, two identical nucleons (protons or neutrons) cannot occupy the same quantum state. However, if we introduce a $\Lambda$ hyperon inside of the nucleus, it can access any of the states, even the ones that are completely 
filled up with nucleons. In other words, the strangeness degree of freedom allows the $\Lambda$ to coexist with the nucleons on the same energy level. As a matter of fact, the energy levels of $\Lambda$ and $\mathrm{N}$, which have the same quantum number, are shifted with respect to each other. The free $\Lambda \mathrm{N}$ interaction is comparably weaker than $\mathrm{NN}$ interaction. Consequently, in a mean field theory, the resulting $\Lambda$ potential well is shallower in comparison with the depth of the $\mathrm{N}$ potential well. Further, in the nuclear medium the $\Lambda \mathrm{N}$ interaction is affected by the presence of the other nucleons, for example three body forces [2]. The hyperon implanted into the nucleus behaves like a "marked" nucleon. The studies of the continuum states, whose configurations are closely related to that of the target nucleus, are of particular interest. Near recoilless $\Lambda$ production is necessary for the investigation of such states.

\section{$1.2 \Lambda$ decay modes and lifetime}

Because the $\Lambda$ has isospin $I=0$, meson exchange with $\Delta I=1$ in the $\Lambda \mathrm{N}$ system is impossible [1]. In such cases the isospin is not conserved at the $\Lambda \Lambda \pi$ vertex (Fig. 1.2.1.a). The exchange is viable for zero isospin mesons and strange mesons, Fig. 1.2.1.b and 1.2.1.c.

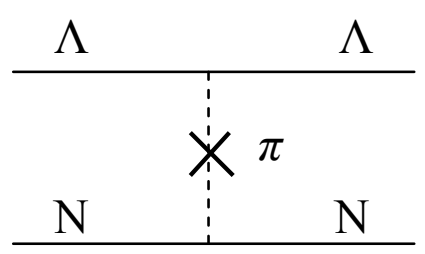

Figure 1.2.1.a Prohibited $\pi$ exchange in $\Lambda-\mathrm{N}$ system.

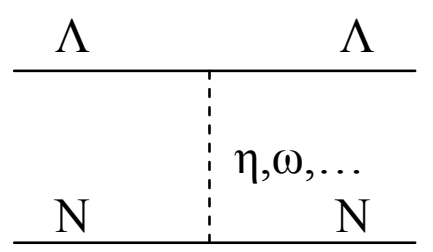

Figure 1.2.1.b Zero isospin meson exchange in $\Lambda-\mathrm{N}$ system.

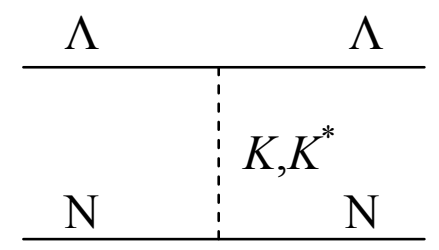

Figure 1.2.1.c Strange meson exchange in $\Lambda-\mathrm{N}$ system. 
The range of an interaction is inversely proportional to the mass of the exchanged boson. With the $\pi$ being the lightest in the meson group, the missing one pion exchange explains the smaller range of the $\Lambda \mathrm{N}$ interaction in comparison to the $\mathrm{NN}$ interaction. Because strangeness is conserved in the strong interaction, the $\Lambda$ particle cannot decay strongly. Free $\Lambda$ hyperons decay weakly via meson production, $\Lambda \rightarrow \mathrm{N}+\pi+40 \mathrm{MeV}$, where strangeness is not conserved. The reaction can proceed in two possible ways: $\Lambda^{0} \rightarrow p^{+}+$ $\pi^{-}$or $\Lambda^{0} \rightarrow n^{0}+\pi^{0}$. The energy released in such a decay is approximately $Q=40 \mathrm{MeV}$. If the $\Lambda$ hyperon was at rest, the energy of the released nucleon would be around $5 \mathrm{MeV}$. If the lower energy levels inside a nucleus are completely filled, such a decay mode would be impossible. The nucleon released in meson decay would not have enough energy to reach the higher empty levels (Fig. 1.2.2.a) [1]. However, there is some probability of such meson decay because of nucleon Fermi motion and high-momentum components of the $\Lambda$ wave function. In spite of the fact that the Pauli principle suppresses meson decay, an additional weak decay mode is possible: $\Lambda+\mathrm{N} \rightarrow 2 \mathrm{~N}+175 \mathrm{MeV}$. It is suitable for nuclei with $\mathrm{Z} \geq 2$.
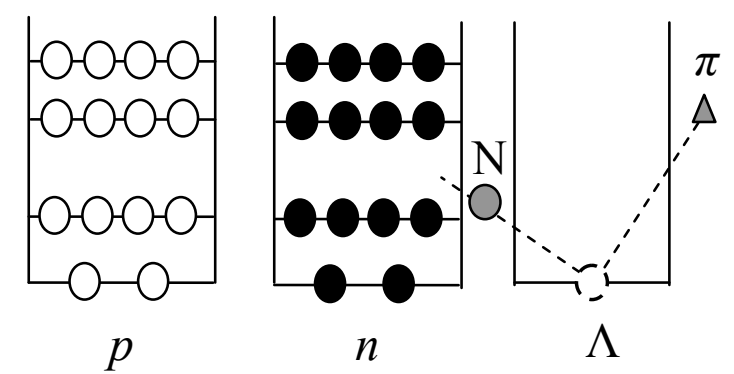

Figure 1.2.2.a Blocked mesonic decay of $\Lambda$ with low energy of released nucleon.

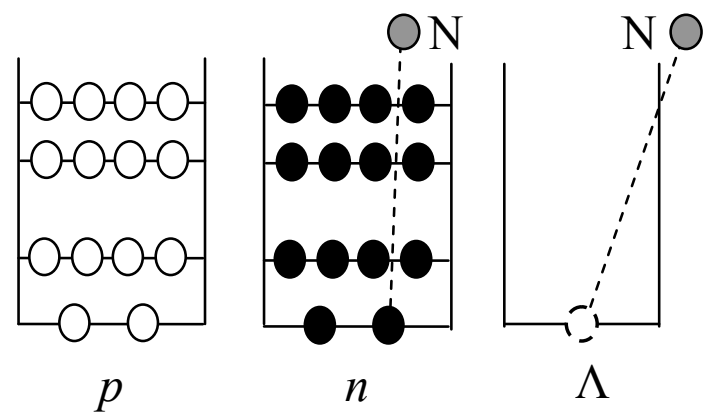

Figure 1.2.2.b Non-mesonic decay of $\Lambda$ with high energetic recoiled nucleons. 
The $175 \mathrm{MeV}$ of energy released in this $2 \mathrm{~N}$ decay reaction gets equally split between the two nucleons. That amount of energy is sufficient for nucleons to overcome Pauli blocking by accessing the higher unoccupied states (Fig. 1.2.2.b). Thus, pion decay dominates mostly in the lightest hypernuclei, for example ${ }_{\Lambda}^{3} \mathrm{H},{ }_{\Lambda}^{4} \mathrm{H}$, and ${ }_{\Lambda}^{4} \mathrm{He}$. The nonmesonic decay mode is apparently more common for heavier hypernuclei and for that reason it determines the $\Lambda$ lifetime. The $\Lambda$ lifetime inside of a nucleus is very close to that of a free one. Currently the tabulated value is about $2.63 \cdot 10^{-10} \mathrm{~s}$ [3]. Time in the nuclear scale is defined by the period of time that it takes for a nucleon to pass through the total diameter of the nucleus $\left(\approx 10^{-23} \mathrm{~s}\right)$. In this sense, the $\Lambda$ particle has a long lifetime and therefore the hypernuclei are considered to be stable. That gives enough time to perform a spectroscopic analysis and investigate electromagnetic properties of hypernuclei.

The heavier hyperons, apart from decaying weakly in a free state, (see Table 1.1.1), are not stable inside of the nucleus. They experience a strong decay via the following conversion reactions:

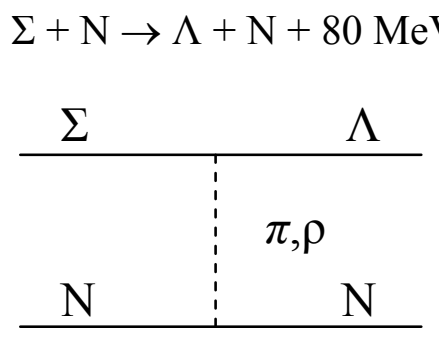

Figure 1.2.3.a $\Sigma$ to $\Lambda$ conversion.

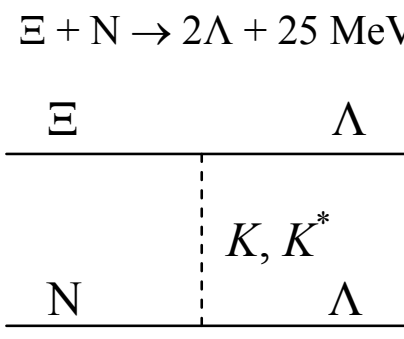

Figure 1.2.3.b $\Xi$ to $\Lambda$ conversion.
$\Omega+\mathrm{N} \rightarrow \Xi+\Lambda+180 \mathrm{MeV}$

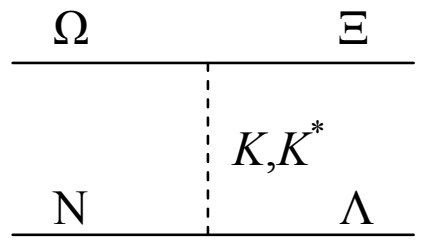

Figure 1.2.3.c $\Omega$ to $\Lambda$ conversion.

Because of the high energy released in these processes, these decays are very rapid - in the range of $10^{-23}$ to $10^{-24}$ seconds. For that reason, in comparison with weak decay 
modes (Table 1.1.1), the lifetimes of $\Sigma, \Xi$, and $\Omega$ are shorter in the presence of nuclear matter. In the third reaction (Fig. 1.2.3.c) the $\Omega$ hyperon converts into a $\Xi$, which then further interacts with a nucleon producing two additional $\Lambda$ particles. That is equivalent to a $\Omega+2 \mathrm{~N} \rightarrow 3 \Lambda$ reaction. As we can see all three types of heavier hyperons quickly decay to $\Lambda$. That makes $\Lambda$ hypernuclei the most easy to study experimentally. However, in spite of that, in special cases one can have $\Sigma$ and $\Xi$ hyperons bound in the nucleus for a long enough time to enable the identification.

Since the $\Lambda$ hypernucleus turns out to be the most stable among the possible hypernuclei, research has been mainly focused on $\Lambda$ hypernuclei. Up to the current moment about 37 types of hypernuclei have been found (Fig. 1.2.4) [4]. The methods used to obtain this chart will be discussed in Section 1.5.

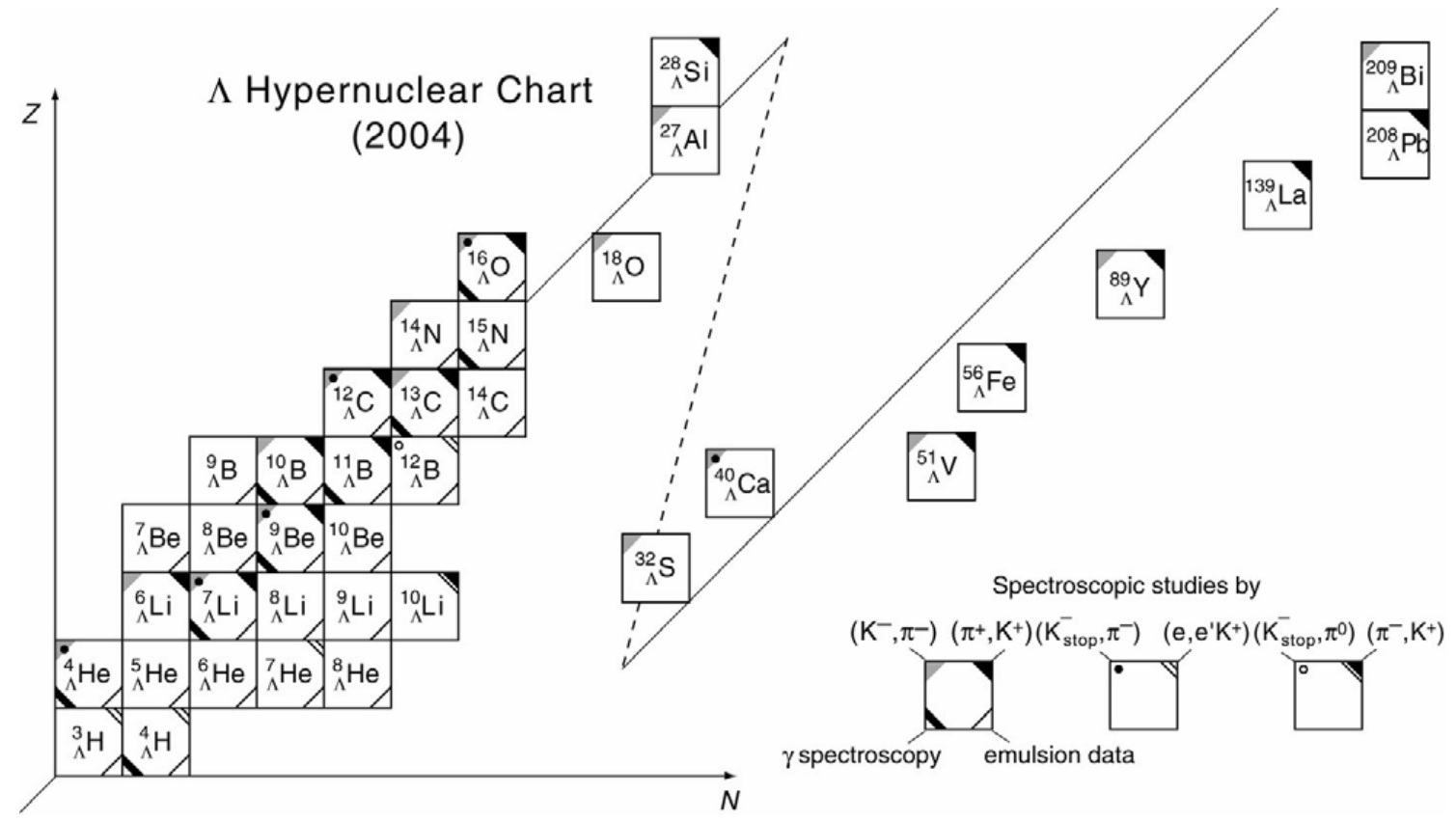

Figure 1.2.4 Chart of $\Lambda$ hypernuclei experimentally found since 1959 [4]. 


\subsection{Binding Energy}

The amount of energy required to break the nucleus of an atom into its isolated nucleons is called the nuclear binding energy. As was mentioned earlier, the study of the $\Lambda \mathrm{N}$ interaction plays an important role in hypernuclear physics. Because of hypernuclear physics' emphasis on the $\Lambda$ particle, it is more convenient to use the separation energy of the $\Lambda$ particle from its nuclear carrier than the binding energy in its classic definition. In the literature this is referred to as $\Lambda$-binding energy, which for the ground state is defined by

$$
B_{\Lambda}(g s)=M_{A}+M_{\Lambda}-M_{Y}
$$

where $M_{Y}$ is the mass of the hypernucleus and $M_{A}$ is merely the mass of the nucleus in the ground state left over after removing the $\Lambda$ particle of mass $M_{\Lambda}$.

The binding energy of the $\Lambda$ particle in its ground state delivers important information on the $\Lambda \mathrm{N}$ interaction. It can set the reference with respect to which the energy of excited states has to be measured. There is a large number of observed hypernuclear decays that take place from the ground state which reflects the hypernuclei in Fig. 1.2.4. The analysis of kinematics of decay fragments in nuclear emulsions is one of the best methods of determining the binding energy. However, only decays with charged mesons and all other fragments producing visible emulsion tracks can be considered for a $B_{\Lambda}$ measurement in emulsions. Reliable resolution of such decay fragments can only be achieved for light hypernuclei. The decay of heavier $(A>16)$ hypernuclei cannot be identified uniquely. In such cases, the counter detector 
experiments, which employ hypernuclear production reactions, are more suitable. The different mechanisms of hypernuclear production will be described in Section 1.5.2. The experimentally found $\Lambda$ binding energies of hypernuclei are briefly summarized in Table 1.3.1 [4-7].

Table 1.3.1 Experimental $B_{\Lambda}$ in the ground state [4-7].

\begin{tabular}{cccc}
\hline Hypernucleus & $B_{\Lambda} \pm \Delta B_{\Lambda}, \mathrm{MeV}$ & Hypernucleus & $B_{\Lambda} \pm \Delta B_{\Lambda}, \mathrm{MeV}$ \\
\hline${ }_{\Lambda}^{3} \mathrm{H}$ & $0.13 \pm 0.05$ & ${ }_{\Lambda}^{11} \mathrm{~B}$ & $10.24 \pm 0.05$ \\
${ }_{\Lambda}^{4} \mathrm{H}$ & $2.04 \pm 0.04$ & ${ }_{\Lambda}^{12} \mathrm{~B}$ & $11.37 \pm 0.06$ \\
${ }_{\Lambda}^{4} \mathrm{He}$ & $2.39 \pm 0.03$ & ${ }_{\Lambda}^{12} \mathrm{C}$ & $10.76 \pm 0.19$ \\
${ }_{\Lambda}^{5} \mathrm{He}$ & $3.12 \pm 0.02$ & ${ }_{\Lambda}^{13} \mathrm{C}$ & $11.69 \pm 0.12$ \\
${ }_{\Lambda}^{6} \mathrm{He}$ & $4.18 \pm 0.10$ & ${ }_{\Lambda}^{14} \mathrm{C}$ & $12.17 \pm 0.33$ \\
${ }_{\Lambda}^{8} \mathrm{He}$ & $7.16 \pm 0.70$ & ${ }_{\Lambda}^{14} \mathrm{~N}$ & $12.17 *$ \\
${ }_{\Lambda}^{6} \mathrm{Li}$ & $4.50 *$ & ${ }_{\Lambda}^{15} \mathrm{~N}$ & $13.59 \pm 0.15$ \\
${ }_{\Lambda}^{7} \mathrm{Li}$ & $5.58 \pm 0.03$ & ${ }_{\Lambda}^{16} \mathrm{O}$ & $12.42 \pm 0.05$ \\
${ }_{\Lambda}^{8} \mathrm{Li}$ & $6.80 \pm 0.03$ & ${ }_{\Lambda}^{28} \mathrm{Si}$ & $16.60 \pm 0.20$ \\
${ }_{\Lambda}^{9} \mathrm{Li}$ & $8.50 \pm 0.12$ & ${ }_{\Lambda}^{32} \mathrm{~S}$ & $17.50 \pm 0.50$ \\
${ }_{\Lambda}^{7} \mathrm{Be}$ & $5.16 \pm 0.08$ & ${ }_{\Lambda}^{40} \mathrm{Ca}$ & $20.00 \pm 0.50$ \\
${ }_{\Lambda}^{8} \mathrm{Be}$ & $6.80 \pm 0.05$ & ${ }_{\Lambda}^{51} \mathrm{~V}$ & $19.50 *$ \\
${ }_{\Lambda}^{9} \mathrm{Be}$ & $6.71 \pm 0.04$ & ${ }_{\Lambda}^{56} \mathrm{Fe}$ & $21.00 *$ \\
${ }_{\Lambda}^{10} \mathrm{Be}$ & $9.11 \pm 0.22$ & ${ }_{\Lambda}^{89} \mathrm{Y}$ & $23.10 \pm 0.50$ \\
${ }_{\Lambda}^{9} \mathrm{~B}$ & $8.29 \pm 0.18$ & ${ }_{\Lambda}^{19} \mathrm{Y}$ & $24.50 \pm 1.20$ \\
${ }_{\Lambda}^{10} \mathrm{~B}$ & $8.89 \pm 0.12$ & ${ }^{20} \mathrm{La}$ & $26.30 \pm 0.80$ \\
\hline
\end{tabular}

* Uncertainties are not reported

The binding energy $B_{\Lambda}$ grows with increasing number of nucleons. For light hypernuclei the growth rate is about $1 \mathrm{MeV}$ per added nucleon, which slowly decreases with increasing mass until it reaches the asymptotical value $B_{\Lambda}(A \rightarrow \infty) \approx 30 \mathrm{MeV}$ (Fig. 1.3.1). This saturation value is set by the depth of the potential well of the $\Lambda$ particle 
inside the nucleus. Since the nucleon potential well inside of the nucleus is approximately a factor of two deeper than observed for $\Lambda$ 's, it becomes clear that the $\Lambda \mathrm{N}$ attraction is comparably weaker than that of NN. The behavior of the binding energy with respect to atomic number is depicted in the figure below.

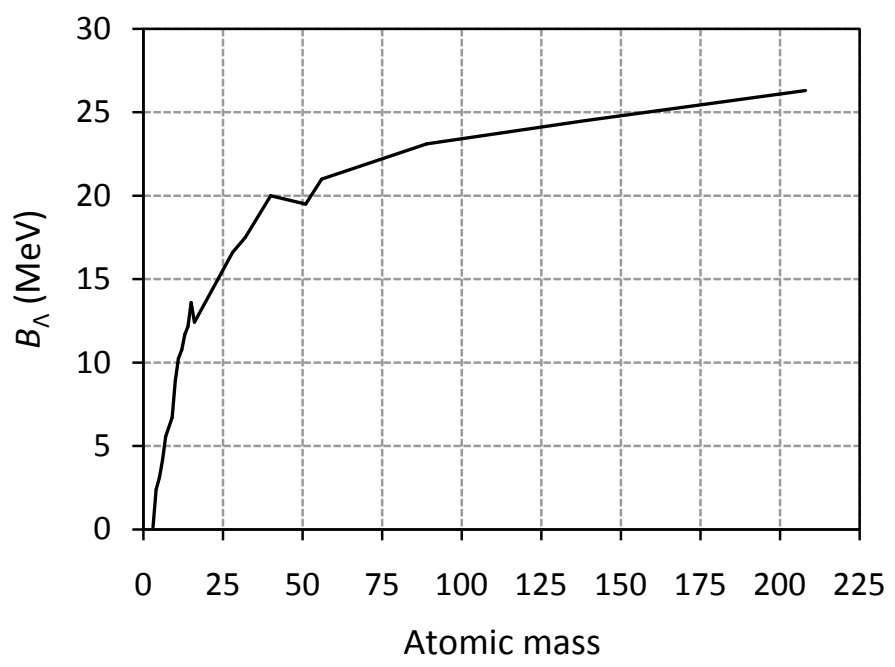

Figure 1.3.1 $\Lambda$ binding energy as a function of atomic number (data from Table 1.3.1).

Free of Pauli blocking, the $\Lambda$ particle inside of the nucleus can occupy any available state, such as the ground state or any excited one. The $\Lambda$ in the ground state will be denoted by $s_{\Lambda}$ state, and the excited states will be denoted by $p_{\Lambda}, d_{\Lambda}, f_{\Lambda}$, and $g_{\Lambda}$ subsequently. A more detailed description of the shell model theory related to these states will be provided in Section 1.4. In the case of the light hypernuclei, the carrier nuclei have a relatively shallow potential well that can only accommodate a few states, for example $s$ and $p$. The $\Lambda$ embedded in such a nuclear system therefore cannot access more states than the nucleus can offer. With an increase of the mass number $A$, the potential well becomes deeper, which allows the hypernucleus to have a higher number of excited 
states. Table 1.3.2 summarizes the experimentally found $\Lambda$ binding energies for different states up to the $g$-shell.

Table 1.3.2 Experimental $B_{\Lambda}$ for heavy hypernuclei $(A \geq 12)$ in ground and excited states [8].

\begin{tabular}{cccccc}
\hline Hypernucleus & $B_{\Lambda}(s), \mathrm{MeV}$ & $B_{\Lambda}(p), \mathrm{MeV}$ & $B_{\Lambda}(d), \mathrm{MeV}$ & $B_{\Lambda}(f), \mathrm{MeV}$ & $B_{\Lambda}(g), \mathrm{MeV}$ \\
\hline${ }_{\Lambda}^{12} \mathrm{C}$ & $10.80 \pm 0.01$ & $0.10 \pm 0.50$ & & & \\
${ }_{\Lambda}^{13} \mathrm{C}$ & $11.70 \pm 0.01$ & $0.80 \pm 0.50$ & & & \\
${ }_{\Lambda}^{16} \mathrm{O}$ & $12.50 \pm 0.35$ & $2.50 \pm 0.50$ & & & \\
${ }_{\Lambda}^{28} \mathrm{Si}$ & $16.60 \pm 0.20$ & $7.00 \pm 0.20$ & & & \\
${ }_{\Lambda}^{32} \mathrm{~S}$ & $17.50 \pm 0.50$ & $8.10 \pm 0.60$ & & & \\
${ }_{\Lambda}^{40} \mathrm{Ca}$ & $18.70 \pm 1.10$ & $11.0 \pm 0.60$ & $1.00 \pm 0.50$ & & \\
${ }_{\Lambda}^{51} \mathrm{~V}$ & $19.90 \pm 1.00$ & & $4.00 \pm 0.50$ & & \\
${ }_{\Lambda}^{89} \mathrm{Y}$ & $23.10 \pm 0.50$ & $16.50 \pm 4.10$ & $9.10 \pm 1.30$ & $2.30 \pm 1.20$ & \\
${ }^{139} \mathrm{La}$ & $24.50 \pm 1.20$ & $20.40 \pm 0.60$ & $14.30 \pm 0.60$ & $8.00 \pm 0.60$ & $1.50 \pm 0.60$ \\
${ }_{\Lambda}^{208} \mathrm{~Pb}$ & $26.30 \pm 0.80$ & $21.90 \pm 0.60$ & $16.80 \pm 0.70$ & $11.70 \pm 0.60$ & $6.60 \pm 0.60$ \\
\hline
\end{tabular}

One can find a slight variation between Tables 1.3.1 and 1.3.2 with respect to binding energies in the ground state $(s)$. That is because the values for $B_{\Lambda}$ were found via different hypernuclear production reactions. Most of the light hypernuclei with mass number $4 \leq A \leq 16$ were obtained by $\left(K^{-}, \pi^{-}\right)$reaction. This reaction also allowed producing hypernuclei with the $\Lambda$ occupying states beyond the $p$-shell. However, the precise determination of the ground state energy for such heavy hypernuclei was not possible. This complication was resolved by introduction of $\left(\pi^{+}, K^{+}\right)$reaction, which gave us most of the current binding energy data, presented in Table 1.3.2.

The dependence of the binding energy $B_{\Lambda}$ for different $\Lambda$ shells on mass number $A$ is shown in Fig. 1.3.2 $[9,10]$. The solid curves correspond to different states and result from a calculation using an effective, density-dependent potential for the $\Lambda \mathrm{N}$ interaction. 
As one can see from the figure, the separation of the $\Lambda$ shells is in the range of $5-10$ $\mathrm{MeV}$ across the periodic table. In order to observe the core exited states, where the core nucleus is in an excited state and the $\Lambda$ in a particular shell, an energy resolution of less than $1 \mathrm{MeV}$ is required.

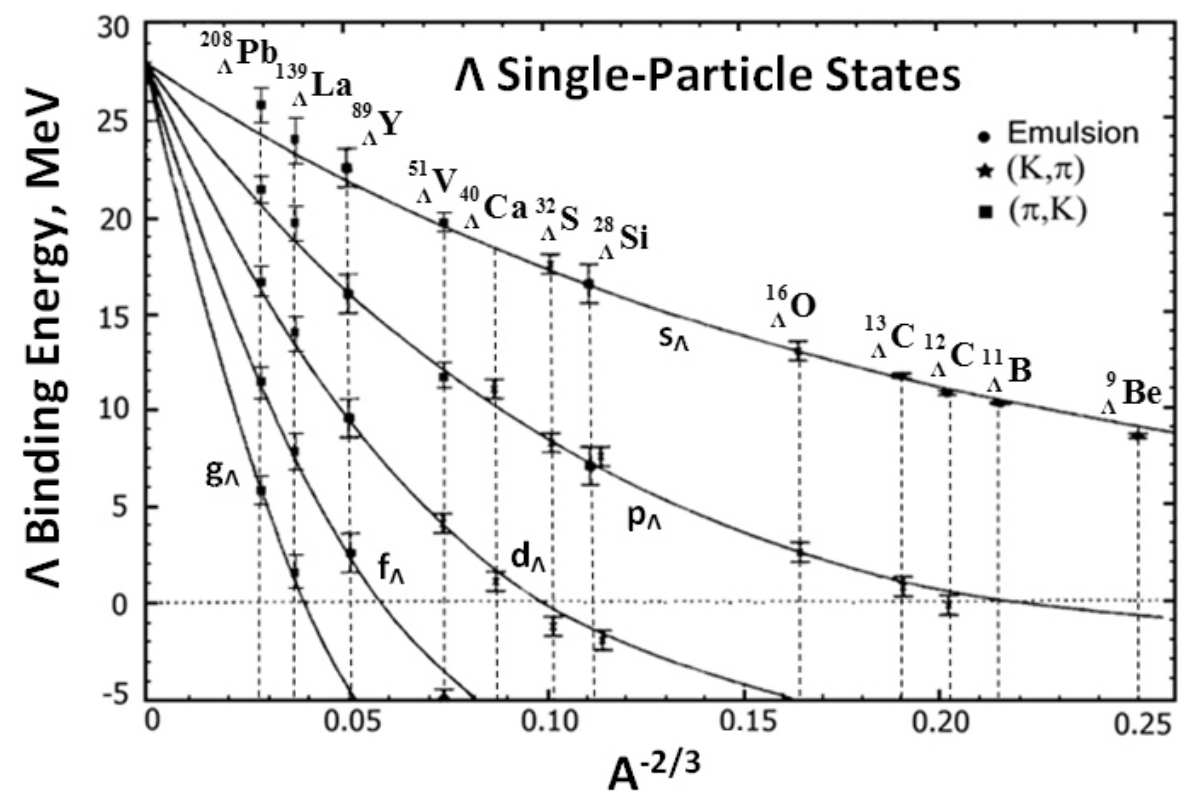

Figure 1.3.2 $\Lambda$ binding energy as a function of atomic number $[9,10]$. The figure was taken from reference [9] and modified to include the data for recently found hypernuclei. 


\subsection{Nuclear Shell Model}

Atomic nuclei are complex and diverse physical objects with many interesting properties. To understand those properties and learn to predict the behavior of the nucleus in different situations, a theory of the nucleus is needed. At the current moment there is no unique theory to explain all the behavioral and structural aspects of the nucleus. One can define two groups of the questions needed to be solved: 1) nuclear structure; 2) mechanism of nuclear reactions and interactions. To answer those, different theories were built, integrated by the statement of quasi-independence of degrees of freedom of the nucleus. The degrees of freedom of the nucleus could be single-particle (independent) and multi-particle (collective). In the independent particle model we assume that no or negligibly little interaction occurs between the individual particles inside of the nucleus. The nucleons in such a model move independently from each other and only experience the influence of the common (mean) field. The shell nuclear model, taking advantage of Bohr's atomic model of electron energy levels, was successfully developed on the idea of this single-particle degree of freedom. It was independently proposed in the late 1940s by physicists M. Goeppert-Mayer and J.H. Jensen, who received the Nobel Prize in 1963 for their work.

It was observed that the nuclides with a certain number of protons or neutrons have an outstandingly high binding energy, in other words are exceptionally stable. These numbers are: $2,8,20,50,82$, and 126 . They are called magic numbers. The nuclei with a magic number of protons and at the same time a magic number of neutrons are called doubly magic nuclei. The latter have the strongest binding energies and therefore are the 
most stable. The existence of magic numbers in nuclei was explained by the shell model theory, where the nucleons - by analogy with the atomic shell model - are suggested to move in orbits. The orbits form shells, just as the orbits of electrons in atoms do. The nucleons are confined inside of a narrow (equivalent to the size of the nucleus $\approx 10^{-14} \mathrm{~cm}$ ) and deep $(\approx 40 \mathrm{MeV})$ potential well. In the first approximation the potential well could be described by an isotropic harmonic oscillator potential $V(r)=-V_{0}+m \omega^{2} r^{2} / 2$. However, the oscillator potential does not really reflect the real shape of the nuclear potential. The more realistic potential, called Woods-Saxon potential, correctly accounts for the smeared profile of the potential edge. It presumes that a given nucleon moves in an effective attractive field formed by all the other nucleons. The expression for the WoodsSaxon potential is therefore proportional to the density distribution and it has the following form: $V_{\text {ws }}=-V_{0} /\left(1+e^{(r-R) / a}\right)$, where $V_{0}$ is the depth of the well, $R$ is the radius of the nucleus, and $a$ is the edge diffusion parameter of the potential well.

According to quantum mechanics, nucleons under the influence of such a potential can occupy discrete energy states. For each nucleon one can assign the total angular momentum $\vec{J}$, which is composed of the sum of the orbital angular momentum $\vec{L}$ and the nucleon's spin $\vec{S},(\vec{J}=\vec{L}+\vec{S})$. All nucleons (protons and neutrons) are fermions with spin $S=1 / 2$. They obey the Pauli principle, which prohibits two identical particles from occupying the same quantum state. Because of the spherical symmetry of the potential and the isotropy of the field, the $2 l+1$ possible orientations of the vector $\vec{L}$ possess the same value of energy. Therefore on the same energy level one can place 
$2(2 l+1)$ of nucleons of the same type. The coefficient 2 in front of the bracket results from the two orientations of the spin.

It is worth mentioning that the Woods-Saxon potential still does not effectively explain the existence of higher magic numbers. The successful solution to this problem was found through implementing the spin-orbit coupling term ( $l s$ term) into the expression of the potential. It becomes:

$$
V(r)=V_{\mathrm{ws}}(r)+\frac{V_{\mathrm{ls}}\langle l \mid s\rangle}{\hbar^{2}} .
$$

The spin-orbit interaction is caused by an interaction of a nucleon's spin with its angular momentum. The $l$ and $s$ values of a nucleon combine to form total angular momentum $j$, and these $j$ values of different nucleons interact producing so called $j$ - $j$ coupling. The spin-orbit term $V_{1 \mathrm{~s}}$ is negative and it leads to an energy split, which is directly proportional to orbital angular momentum as $2 l+1$. Because of the energy split, the total angular momentum quantum number $j$ acquires two possible values: $j_{ \pm}=l \pm 1 / 2$. It was found experimentally that for the anti-parallel spin-orbit orientation $j_{-}=l-1 / 2$ the energy change is positive. In case of parallel spin-orbit configuration, the energy decreases making the $j_{+}=l+1 / 2$. The distances between the energy levels with $j_{-}$and $j_{+}$are in the $\mathrm{MeV}$ range. For higher $l$ the splitting increases, and for $l \geq 4$ the levels get separated so much that they reach into neighboring shells (Fig. 1.4.1). By a shell we understand a group of closely spaced energy levels. The number of nucleons occupying 
the individual level are calculated to be $m=2 j+1$. Table 1.4.1 shows the level distribution inside of the shells. From Fig. 1.4.1 and Table 1.4.1 we see that the accumulated nucleon occupancy at the end of each shell coincide with a magic number. At the same time the shells have a wide energy separation $\left(\approx \hbar \omega \approx 41 \mathrm{~A}^{-1 / 3} \mathrm{MeV}\right)$. That explains the high nuclear stability of the magic nucleus in its ground state, where all available states are occupied. Note that the relative position of the energy levels in Fig. 1.4.1 might change depending on the nucleon of interest (i.e. proton or neutron).

Table 1.4.1 Level distribution in the shells of the nucleus (Woods-Saxon potential with spin-orbit coupling).

\begin{tabular}{llllll}
\hline Shell $\#$ & Levels & Parity & States & \# of nucleons \\
& & & $m=2 j+1$ & $\sum m$ \\
\hline I $(l=0)$ & $1 \mathrm{~s}$ & + & $1 s_{1 / 2}$ & 2 & 2 \\
\hline II $(l=1)$ & $1 \mathrm{p}$ & - & $1 p_{3 / 2} 1 p_{1 / 2}$ & $4+2=6$ & 8 \\
\hline III $(l=0,2)$ & $2 \mathrm{~s} 1 \mathrm{~d}$ & + & $1 d_{5 / 2} 2 s_{1 / 2} 1 d_{3 / 2}$ & $6+2+4=12$ & 20 \\
\hline IV $(l=1,3,4)$ & $2 \mathrm{p} 1 \mathrm{f} 1 \mathrm{~g}^{*}$ & - & $1 f_{7 / 2} 2 p_{3 / 2} 1 f_{5 / 2} 2 p_{1 / 2} 1 g_{9 / 2}$ & $8+4+6+2+10=30$ & 50 \\
\hline V $(l=0,2,4,5)$ & $3 \mathrm{~s} 2{\mathrm{~d} 1 \mathrm{~g} 1 \mathrm{~h}^{*}}^{*}+$ & $1 g_{7 / 2} 2 d_{5 / 2} 2 d_{3 / 2} 3 s_{1 / 2} 1 h_{11 / 2}$ & $8+6+4+2+12=32$ & 82 \\
\hline VI $(l=1,3,5,6)$ & $3 \mathrm{p} 2 \mathrm{f} 1 \mathrm{~h} 1 \mathrm{i}^{*}$ & - & $1 h_{9 / 2} 2 f_{7 / 2} 2 f_{5 / 2} 3 p_{3 / 2} 3 p_{1 / 2} 1 i_{13 / 2}$ & $10+8+6+4+2+14=44$ & 126 \\
\hline
\end{tabular}

(The ${ }^{*}$ represent a level overlap by a neighbor shell) 


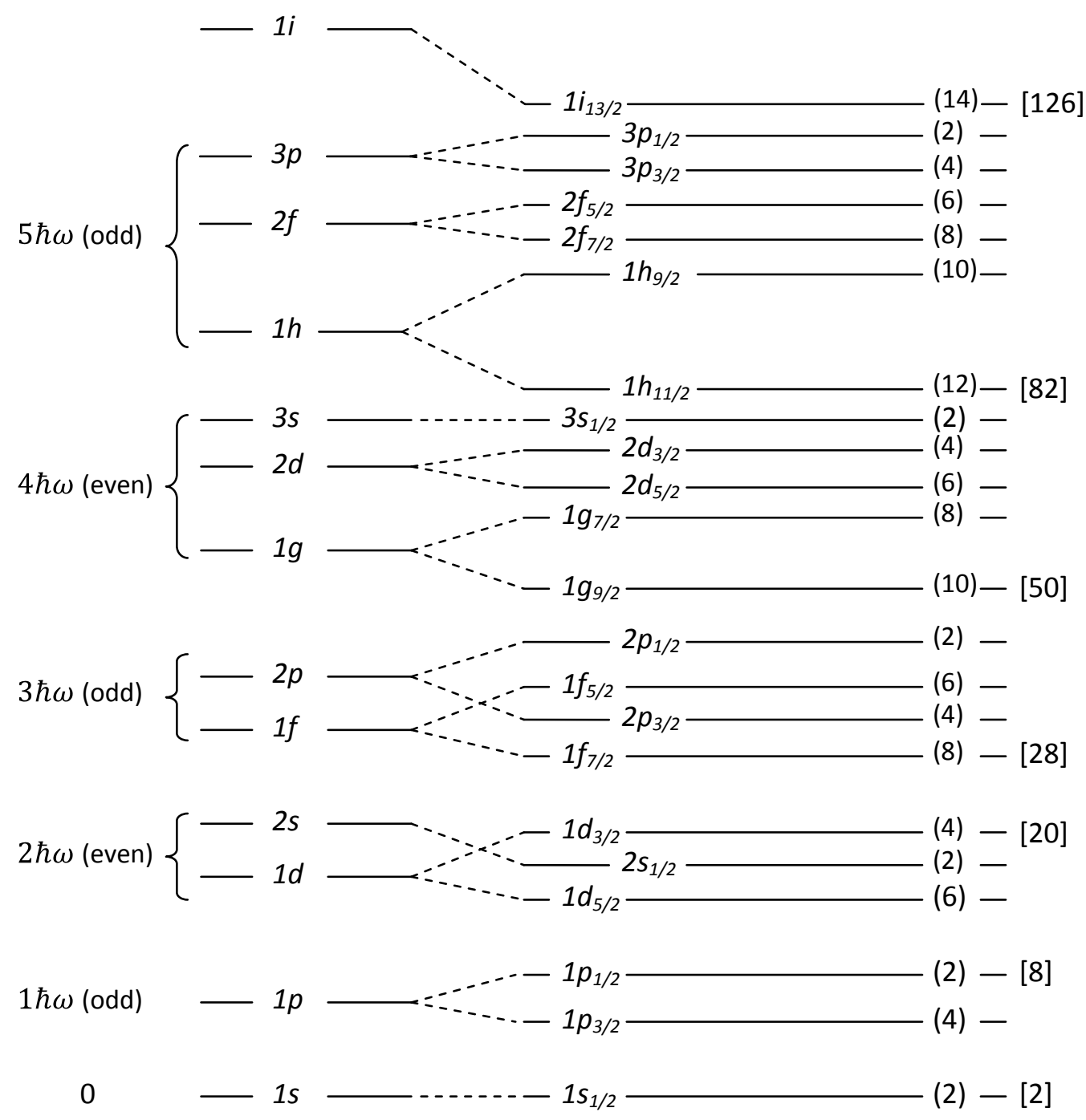

$\begin{array}{lllll}\text { Harmonic } & \text { Reasonable Nuclear } & \text { Reasonable Nuclear } & \text { Level } & \text { Magic } \\ \text { Oscillator } & \text { (Woods-Saxon) } & \text { with spin-orbit term } & \text { Occupancy } & \text { Numbers }\end{array}$

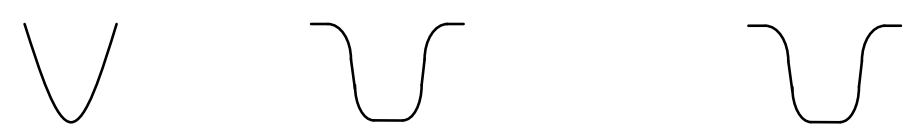

Figure 1.4.1 Single-particle energy levels in the nuclear central potential (Woods-Saxon). The spin-orbit coupling is taken into account on the right and ignored on the left. The brackets on the left unite the levels into one harmonic oscillator shell. 


\section{$1.5 \Lambda$ hypernuclear production}

\subsubsection{Historical overview}

In the late 1940 s and 1950 s of the previous century the concept of strangeness was not completely understood. The strange particles were repeatedly observed in emulsion experiments, but the concept of a new conserved quantum number was not acknowledged. The existence of nuclei containing such kind of strange particle was not rejected, however in spite of that the idea was not seriously explored. The discovery of the first nucleus with an embedded strange particle came very unexpectedly. In 1952, two Polish scientists, M. Danysz and J. Pniewski were studying cosmic rays with photoemulsion material [11]. They obtained an interesting photo (Fig.1.5.1).

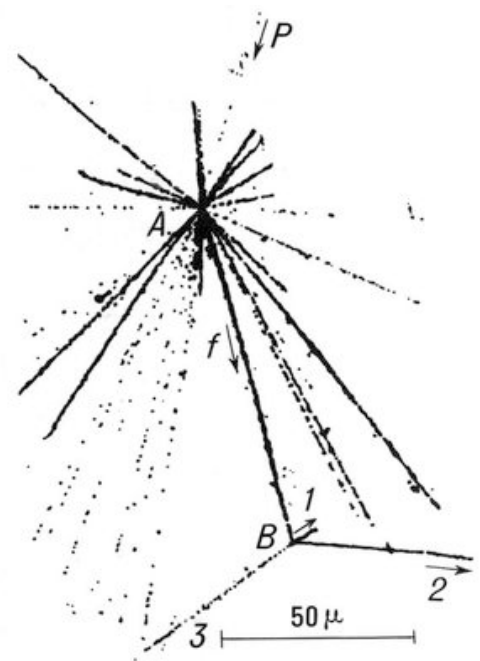

Figure 1.5.1 Emulsion photo of the first hypernuclear event [11].

The cosmic particle $P$ collided at point $A$ with a nucleus in the emulsion producing the hypernuclear $\operatorname{track} f$, which afterwards decayed into three tracks: 1,2 , and 3 . The analysis 
of the event has lead scientists to recognize the fragment $f$ as a strange nucleus, what we call now a $\Lambda$ hypernucleus.

After the first cosmic ray observations pion and proton beams were utilized for $\Lambda$ production:

$$
p+\mathrm{N} \rightarrow \mathrm{N}+K^{+}+\Lambda \text { and } \quad \pi^{+}+n \rightarrow K^{+}+\Lambda
$$

The subsequent mesonic decay products were detected in emulsions and ${ }^{4} \mathrm{He}$ bubble chambers. Unfortunately these reactions are characterized by small cross sections that cannot always be compensated for by increasing the intensity of the beam. At the same time the emulsion material has its own insufficiency. It contains multiple target nuclei, such as ${ }^{2} \mathrm{H}$, light nuclei of ${ }^{12} \mathrm{C},{ }^{14} \mathrm{~N},{ }^{16} \mathrm{O}$, heavy nuclei of ${ }^{108} \mathrm{Ag}$ and ${ }^{80} \mathrm{Br}$. The inevitable scattering on such secondary targets introduced limitations on emulsion techniques.

The first reliable information about properties of $\Lambda$ hypernuclei was obtained from stopped $K^{-}$absorption reactions:

$$
K^{-}+n \rightarrow \pi^{-}+\Lambda \quad \text { and } \quad K^{-}+p \rightarrow \pi^{0}+\Lambda
$$

Such reactions employed emulsion techniques as well and were carried out at The European Organization for Nuclear Research (CERN) and Brookhaven National Laboratory (BNL). Because the $K$ has the same strangeness, $S=-1$, as the $\Lambda$ hyperon, the probability of hyperon production is relatively high in this reaction. At the same time, unwanted background reactions are reduced. Sometimes the photo-emulsion allows tracking the whole process of hypernuclear production up to the level of formation 
of weak decay products. This technique allowed measuring the binding energies, lifetimes, and spins of most of the light hypernuclei, all essentially in the nuclear $1 s$ and $1 p$ shells with mass number ranging from $3\left({ }_{\Lambda}^{3} \mathrm{H}\right)$ to $15\left({ }_{\Lambda}^{15} \mathrm{~N}\right)$ inclusively.

In the late $1960 \mathrm{~s}$, with the advent of intense $K$ beams, hypernuclear physics entered into a new era of counter detector experiments. At that time spectroscopy was achieved primarily by employing the reaction $K^{-}+n \rightarrow \pi^{-}+\Lambda$ for both stopped and in-flight, low-momentum kaons $(p=400-800 \mathrm{MeV} / \mathrm{c})$. The high intensity of the kaon beam made it possible to study not only bound states, but also hypernuclear continuum states. The in-flight kaon reaction took advantage of nearly recoilless kinematics with very weak spin-flip transitions to achieve unusual hypernuclear excited states. There were a lot of hypernuclear studies by using $\left(K^{-}, \pi^{-}\right)$reaction, particularly for the excited $p$ shell. Since the momentum transfer in this reaction is zero or almost negligible, the incident kaon most likely interacts with an outer shell nucleon replacing the nucleon with a $\Lambda$ captured into the same shell $(\Delta L=0)$. The reaction with negative kaons also allowed achieving heavier hypernuclei as well, however, a definite determination of ground state binding energies was not feasible. It became possible with the application of the $\pi^{+}+n \rightarrow K^{+}+\Lambda$ reaction, which took place in the mid-1980's at BNL and then was adopted by The High Energy Accelerator Research Organization (KEK) and CERN. The reaction allowed measuring with high quality a broad range of hypernuclei in both light and heavy atomic mass regions.

Within the last decade, new exciting hypernuclear spectroscopy techniques have been developed. One of them is hypernuclear $\gamma$-ray spectroscopy. Gamma-ray 
spectroscopy utilized the hypernuclear production reactions $\left(K^{-}, \pi^{-}\right)$and $\left(\pi^{+}, K^{+}\right)$, available at BNL and KEK, and with a few $\mathrm{keV}$ resolution measured the $s-p \gamma$-transition energies. Using Germanium detectors, the Hyperball (1998) and Hyperball2 (2005), experiments uncovered level structures of several light p-shell hypernuclei. Measured level structures of hypernuclei provided new quantitative information on hyperonnucleon interactions. Another intriguing advancement was the realization of a new hypernuclear electroproduction mechanism based on the $\left(e, e^{\prime} K^{+}\right)$reaction. The first successful experiment E89-009 (HNSS) utilizing this reaction was carried out at Thomas Jefferson National Accelerator Facility (JLab) in 2000 [12]. The experiment featured the high-resolution $p$-shell hypernuclear spectra of ${ }_{\Lambda}^{12} \mathrm{~B}$. The measured energy resolution of $\Delta E \approx 700 \mathrm{keV}$ set the record for hypernuclear spectroscopy at that time.

\subsubsection{Production mechanisms}

In the historical overview of hypernuclear production we briefly mentioned the reactions used for formation of $\Lambda$ hypernuclei. In this section each individual reaction will be described in more detail. As I noted earlier, there are many ways to obtain a $\Lambda$ hyperon bound to a nucleus. However, we can define two major types of production mechanisms: strangeness exchange and strangeness production. The corresponding quark flow diagrams are schematically presented in Fig. 1.5.2. Strangeness exchange reactions employ strange mesonic beams, which in the reaction process transfer the strangeness into the nuclear medium. In such reactions both the incident particles and reaction products contain the strangeness degree of freedom. The associated strangeness 
production mechanism is based on $s \bar{s}$-pair production during the reaction inside the nuclear medium, i.e., the strange quark is not present in the incident particle or target. The strangeness is created by the absorption of the virtual photon or pion.

The well known strangeness exchange reactions are mesonic $\left(K^{-}, \pi^{0}\right)$ reactions. The reactions utilizing proton $\left(p, K^{+}\right)$, pion $\left(\pi^{+}, K^{+}\right)$, and electron $\left(e, e^{\prime} K^{+}\right)$beams belong to the strangeness production mechanism. Since some of the mentioned reactions might have different outcome scenarios (channels), we will only review the ones that actually lead to $\Lambda$ hypernuclear production.

Strangeness exchange reaction

$$
K^{-}+n \rightarrow \Lambda+\pi^{-} \quad K^{-}+p \rightarrow \Lambda+\pi^{0}
$$

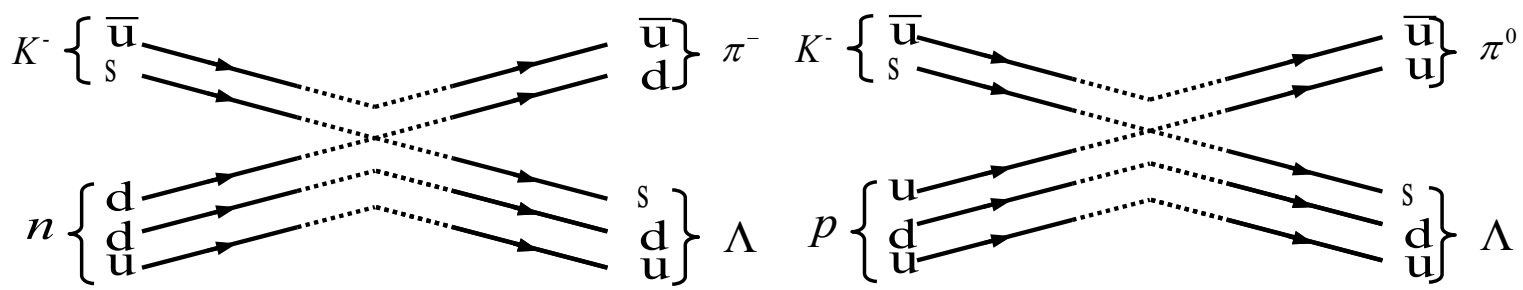

Associated strangeness production

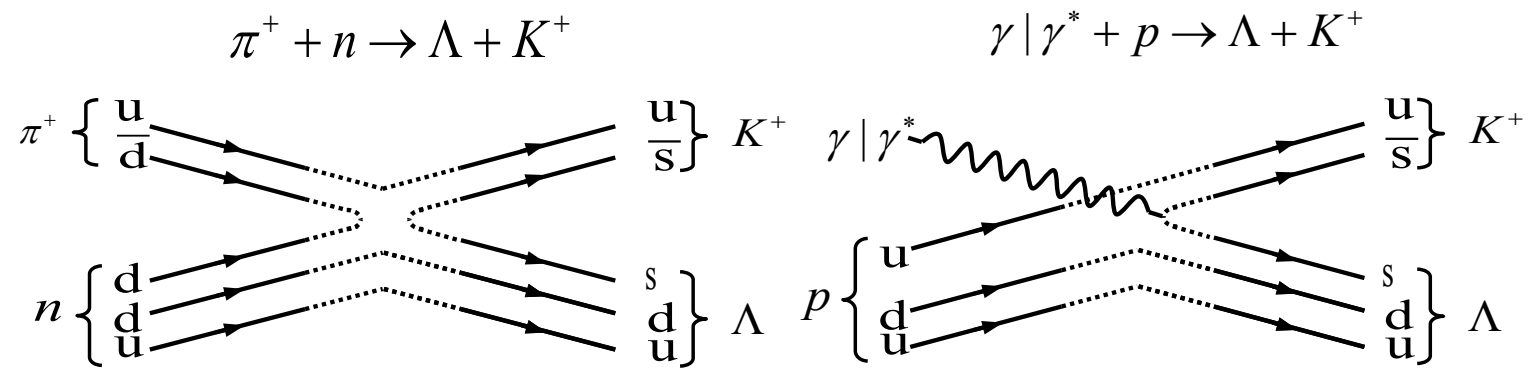

Figure 1.5.2 Quark flow schematic diagrams of $\Lambda$ hypernuclear production. 
The strange mesonic reactions taking advantage of the strangeness exchange mechanism are:

$$
\begin{aligned}
\left(K^{-}+n \rightarrow \Lambda+\pi^{-}\right): & K^{-}+{ }^{\mathrm{A}} Z \rightarrow\left\{\begin{array}{l}
\mathrm{A}-1 \\
(Z)+\Lambda+\pi^{-}, \\
{ }_{\Lambda}^{\mathrm{A}}(Z)+\pi^{-}
\end{array},\right. \\
\left(K^{-}+p \rightarrow \Lambda+\pi^{0}\right): & K^{-}+{ }^{\mathrm{A}} Z \rightarrow\left\{\begin{array}{l}
{ }^{\mathrm{A}-1}(Z-1)+\Lambda+\pi^{0} \\
{ }_{\Lambda}^{\mathrm{A}}(Z-1)+\pi^{0}
\end{array} .\right.
\end{aligned}
$$

The reactions utilizing the associated strangeness production mechanism are:

$$
\begin{aligned}
& \left(p+\mathrm{N} \rightarrow \mathrm{N}+\Lambda+K^{+}\right): \quad p+{ }^{\mathrm{A}} Z \rightarrow\left\{\begin{array}{l}
{ }^{\mathrm{A}}(Z)+\Lambda+K^{+} \\
{ }_{\mathrm{A}+1}(Z)+K^{+} \\
{ }_{\mathrm{A}}(Z-1)+K^{+}+p^{\prime} \\
{ }_{\Lambda}
\end{array}\right. \\
& \left(\pi^{+}+n \rightarrow \Lambda+K^{+}\right): \quad \pi^{+}+{ }^{\mathrm{A}} Z \rightarrow\left\{\begin{array}{l}
\mathrm{A}-1 \\
{ }^{\mathrm{A}}(Z)+\Lambda+K^{+} \\
{ }_{\Lambda}(Z)+K^{+}
\end{array},\right. \\
& \left(\pi^{-}+p \rightarrow \Lambda+K^{0}\right): \quad \pi^{-}+{ }^{\mathrm{A}} Z \rightarrow\left\{\begin{array}{l}
\mathrm{A}-1 \\
{ }_{\mathrm{A}}(Z-1)+\Lambda+K^{0} \\
{ }_{\Lambda}(Z-1)+K^{0}
\end{array}\right. \\
& \left(\gamma+n \rightarrow \Lambda+K^{0}\right) \\
& \gamma+{ }^{\mathrm{A}} Z \rightarrow\left\{\begin{array}{l}
\mathrm{A}-1 \\
{ }_{\Lambda}^{\mathrm{A}}(Z)+\Lambda+K^{0}
\end{array}\right. \\
& \left(\gamma+p \rightarrow \Lambda+K^{+}\right): \quad \gamma+{ }^{\mathrm{A}} Z \rightarrow\left\{\begin{array}{l}
{ }^{\mathrm{A}-1}(Z-1)+\Lambda+K^{+}, \\
{ }_{\Lambda}^{\mathrm{A}}(Z-1)+K^{+},
\end{array}\right. \\
& \left(e+n \rightarrow e^{\prime}+\Lambda+K^{0}\right): \quad e+{ }^{\mathrm{A}} Z \rightarrow\left\{\begin{array}{l}
\mathrm{A}-1 \\
(Z)+\Lambda+K^{0}+e^{\prime} \\
{ }_{\Lambda}^{\mathrm{A}}(Z)+K^{0}+e^{\prime}
\end{array},\right.
\end{aligned}
$$




$$
\left(e+p \rightarrow e^{\prime}+\Lambda+K^{+}\right): \quad e+{ }^{\mathrm{A}} Z \rightarrow\left\{\begin{array}{l}
\mathrm{A}-1(Z-1)+\Lambda+K^{+}+e^{\prime} \\
{ }_{\Lambda}^{\mathrm{A}}(Z-1)+K^{+}+e^{\prime}
\end{array}\right.
$$

The reactions with negative kaons are the most widely used for hypernuclear production and the best studied. Their application started during the photo-emulsion era and merged into the age of the counter detector experiments. It is important to note that reaction (1.5.2), is not suitable for hypernuclear spectroscopy. The neutral pion released in this reaction can only be detected by tracing its decay products, which are two $\gamma$ particles $\left(\pi^{0} \rightarrow 2 \gamma\right)$. The detection of gammas with high resolution is not an easy task. Thus, the commonly used reaction with negative kaons is (1.5.1). It was convenient to use this reaction in early emulsion experiments because of the low number of background reactions in the emulsion material. With utilization of high intensity $K^{-}$beams in counter detector experiments, the spectroscopy of the outgoing $\pi^{-}$gives complete information on the hypernuclear system. One of the main characteristics of the $n\left(K^{-}, \pi^{-}\right) \Lambda$ reaction is the possibility to run kinematics with a low momentum transfer (Fig. 1.5.3). For kaon momenta between 250 and $1250 \mathrm{MeV} / \mathrm{c}$, the recoil momentum of the $\Lambda$ particle is less than $100 \mathrm{MeV} / \mathrm{c}$.

Taking into account that the Fermi momentum of the nucleons in the nucleus is of the order of $250 \mathrm{MeV} / \mathrm{c}$, there is a high chance for the recoiling $\Lambda$ to form a hypernucleus. As a result of a low, or even zero momentum transfer, the incident kaons attenuate rapidly in the nuclear matter. The interaction will mostly occur with a neutron on the outer shell. As a result, the neutron is replaced by a $\Lambda$ hyperon, which obtains the same spin and orbit quantum numbers as the removed nucleon. In this way the $\Lambda$ 
populates so called substitutional states. At forward angles the elementary transition $n \rightarrow \Lambda$ excites low-spin states with natural parity $P=(-1)^{L}$. With the recoil momentum exceeding the nucleons' Fermi momentum, the newborn $\Lambda$ has a greater chance to leave the nucleus; this is called a quasi-free (QF) reaction. In comparison with other $\Lambda$ hypernuclear production processes the $\left(K^{-}, \pi^{-}\right)$process has the biggest cross section at forward angles, on the order of $10 \mathrm{mb} / \mathrm{sr}$.

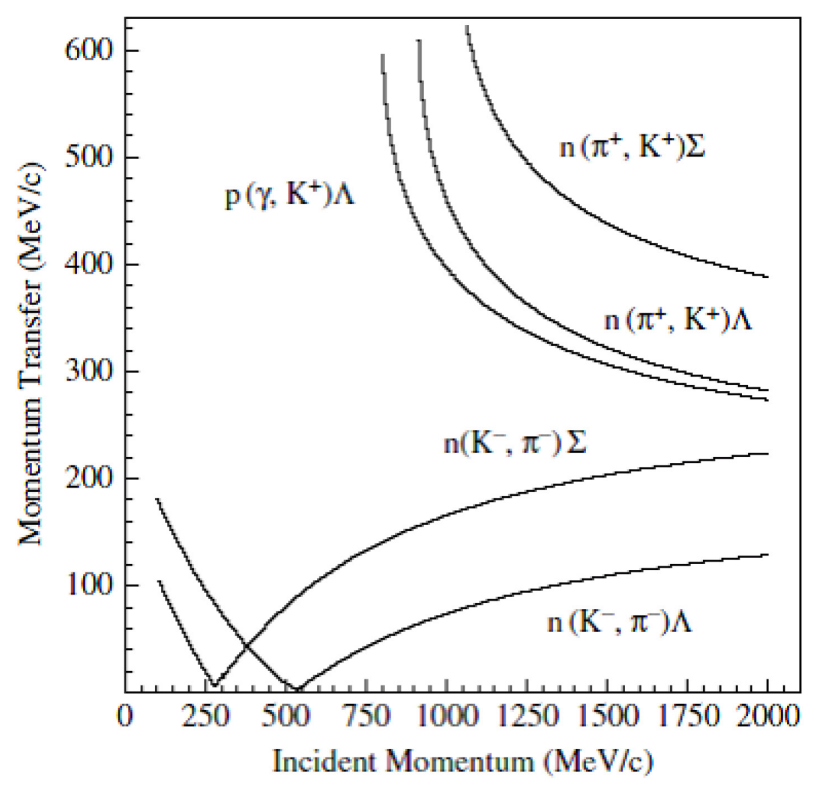

Figure 1.5.3 Hyperon recoil momentum in various elementary reactions at $\theta=0^{\circ}[6]$.

The reaction with incident protons, (1.5.3), is characterized by a much smaller cross section $(\approx 10 \mathrm{nb} / \mathrm{sr})$, which cannot always be compensated for by increased beam intensity. Historically, the emulsion technique was the primary method for studying the products of the mentioned reaction. Then the $\vec{p} p$ scattering process with $\Lambda$ hypernuclear production was intensively studied at The Cooler Synchrotron (COSY), where the $\Lambda p$ 
interaction was the major direction of research. [Note that $\vec{p}$ scattering indicates a polarized proton]. Experimentally, for reactions (1.5.3), it is easier to study the proton scattering on neutrons than protons on protons. In the elementary transition $n \rightarrow \Lambda$, the positive kaon is the only outgoing particle that has to be detected in order to determine the energy of the hypernucleus. The transition $p \rightarrow \Lambda$ complicates the experiment by requiring the two particles, $K^{+}$and $p^{\prime}$, to be measured simultaneously.

As I mentioned before, the reactions on negative kaons were characterized by low momentum transfer. Because of that, the reaction was mostly suitable for light nuclear targets, where only ground, $s$, and first excited state, $p$, are present. For medium and heavy targets a reaction with high momentum transfer, for example $\left(\pi^{+}, K^{+}\right)$, to populate low lying states, especially the ground state, is needed. Such a condition is satisfied in the process (1.5.4), Fig. 1.5.3. The $\left(\pi^{+}, K^{+}\right)$reaction strongly populates deeply-bound, highspin, natural parity states. The energetic $\pi^{+}$and $K^{+}$particles in comparison to low momentum $K^{-}$have longer mean paths in nuclear matter. That favors the high momentum transfer, which in turn compliments the quasi-free processes with the reduction of the cross sections to bound states. Both of the reactions, the $\left(K^{-}, \pi^{-}\right)$and $\left(\pi^{+}, K^{+}\right)$, do not have a significant spin-flip amplitude at forward angles. Apart from the early emulsion experiments, the mesonic hypernuclear production reactions in general achieved hypernuclear spectra with energy resolutions of the order of $2 \mathrm{MeV}$ and more. The best energy resolution obtained so far by the $\left(\pi^{+}, K^{+}\right)$reaction is about $1.5 \mathrm{MeV}$, where a thin ${ }^{12} \mathrm{C}$ target was used with an extended run-time to accumulate significant statistics. 
The reactions of type (1.5.6) - (1.5.9) exploit the associated strangeness production mechanism. Kinematics of the processes $\left(\gamma, K^{+}\right)$and $\left(e, e^{\prime} K^{+}\right)$is almost identical. In the case of incident electrons, hypernuclear production is done via exchange of a virtual photon, while in the other process a real photon is used. Out of two possible reactions, (1.5.8) and (1.5.9), involving an electron beam, the most favorable is the one utilizing the $p \rightarrow \Lambda$ transition. It is experimentally easier to detect positive kaons than tracking the decay products of neutral kaons. In counter detector experiments the indirect high resolution detection of the reaction products, namely the products of the subsequent decay, is almost not possible.

Similar to $\left(\pi^{+}, K^{+}\right)$reaction the $\left(e, e^{\prime} K^{+}\right)$reaction excites the high-spin, bound hypernuclear states. On top of that, because of absorption of a spin 1 virtual photon and high momentum transfer, both natural and unnatural parity (spin-flip) states are produced with comparable strength. By unnatural parity states we understand the states that have been populated by spin-flip $(\Delta J \neq \Delta L)$ transitions and acquired $P=(-1)^{L+1}$ parity. The $\left(e, e^{\prime} K^{+}\right)$reaction has a relatively small cross section of approximately $10 \mathrm{nb} / \mathrm{sr}$. Such a small number can be to some extent compensated by the intensity of the electron beam. It is certainly an easy task for modern continuous electron-beam accelerators. In mesonic hypernuclear production, the low intensity secondary meson beams apparently required the use of relatively thick targets, which degrades the energy of the detected meson. In case of the high intensity electron beam with excellent spatial and energy resolutions, the targets can be physically small and thin $\left(10-50 \mathrm{mg} / \mathrm{cm}^{2}\right)$. The energy resolution in this reaction has the potential to reach a few hundred $\mathrm{keV}$. As we stated before, energy 
resolutions of the order of $700 \mathrm{keV}$ have been reported. In this work we will present comparable numbers (see Chapter 5). The unique features that make the $\left(e, e^{\prime} K^{+}\right)$ reaction worth studying are:

- High probability of spin-flip transitions due to the angular momentum carried by the virtual photon. Such unnatural parity states are suppressed in reactions involving mesons.

- Production of hypernuclei that are not accessible by mesonic reactions. For example, the meson beam on ${ }^{12} \mathrm{C}$ target yields ${ }_{\Lambda}^{12} \mathrm{C}$, while the electron on the same target creates ${ }_{\Lambda}^{12} \mathrm{~B}$, the mirror hypernucleus to ${ }_{\Lambda}^{12} \mathrm{C}$. (In mirror hypernuclei a number of protons and a number of neutrons are mutually interchanged).

The properties of all mentioned $\Lambda$ hypernuclei production reactions are summarized in the Table 1.5.1.

Table 1.5.1 Properties of hypernuclear production reactions.

\begin{tabular}{|c|c|c|c|c|c|c|c|}
\hline Reaction & Transition & $\Delta \mathrm{Z}$ & $\begin{array}{c}\text { Cross } \\
\text { section }(\mathrm{b} / \mathrm{sr})\end{array}$ & $\begin{array}{c}\Lambda \text { momentum } \\
\text { transfer }\end{array}$ & $\begin{array}{c}\text { Populated } \\
\text { states }\end{array}$ & $\begin{array}{l}\text { Production } \\
\text { mechanism }\end{array}$ & $\begin{array}{c}\text { Accelerator } \\
\text { facility }\end{array}$ \\
\hline $\begin{array}{l}\left(K^{-}, \pi^{-}\right) \\
\left(K^{-}, \pi^{0}\right)\end{array}$ & $\begin{array}{l}n \rightarrow \Lambda \\
p \rightarrow \Lambda\end{array}$ & $\begin{array}{c}0 \\
-1\end{array}$ & $10^{-3}$ & low & $\begin{array}{l}\text { substitutional, } \\
\text { low-spin, } \\
\text { natural parity }\end{array}$ & $\begin{array}{c}\text { strangeness } \\
\text { exchange }\end{array}$ & $\begin{array}{l}\text { BNL, } \\
\text { CERN, } \\
\text { KEK }\end{array}$ \\
\hline $\begin{array}{l}\left(p, K^{+}\right) \\
\left(p, K^{+} p^{\prime}\right)\end{array}$ & $\begin{array}{l}n \rightarrow \Lambda \\
p \rightarrow \Lambda\end{array}$ & $\begin{array}{c}0 \\
-1\end{array}$ & $10^{-9}$ & high & & associated & COSY \\
\hline $\begin{array}{l}\left(\pi^{+}, K^{+}\right) \\
\left(\pi^{-}, K^{0}\right)\end{array}$ & $\begin{array}{l}n \rightarrow \Lambda \\
p \rightarrow \Lambda\end{array}$ & $\begin{array}{c}0 \\
-1\end{array}$ & $10^{-6}$ & high & $\begin{array}{l}\text { high-spin, } \\
\text { natural parity }\end{array}$ & associated & BNL, KEK \\
\hline $\begin{array}{l}\left(\gamma, K^{0}\right) \\
\left(\gamma, K^{+}\right)\end{array}$ & $\begin{array}{l}n \rightarrow \Lambda \\
p \rightarrow \Lambda\end{array}$ & $\begin{array}{c}0 \\
-1\end{array}$ & $10^{-9}$ & high & $\begin{array}{l}\text { high-spin, } \\
\text { unnatural parity }\end{array}$ & associated & KEK \\
\hline $\begin{array}{l}\left(e, e^{\prime} K^{0}\right) \\
\left(e, e^{\prime} K^{+}\right)\end{array}$ & $\begin{array}{l}n \rightarrow \Lambda \\
p \rightarrow \Lambda\end{array}$ & $\begin{array}{c}0 \\
-1\end{array}$ & $10^{-9}$ & high & $\begin{array}{l}\text { stretched, } \\
\text { high-spin, } \\
\text { unnatural parity }\end{array}$ & associated & JLab \\
\hline
\end{tabular}




\subsubsection{Kinematics of elementary electroproduction reaction}

The kinematics of the elementary process $e+p \rightarrow e^{\prime}+\Lambda+K^{+}$of hypernuclear electroproduction is shown in Fig. 1.5.4. The initial $e$ and final $e$ e electrons with corresponding momenta $\vec{p}_{e}$ and $\vec{p}_{e^{\prime}}$ define the scattering plane $x z$. The transferred momentum $\vec{q}=\vec{p}_{e}-\vec{p}_{e^{\prime}}$ propagates as a virtual photon along the $z$-axis. The reaction plane is defined by the momentum of the resulting kaon, $\vec{p}_{K}$, and $\Lambda$ hyperon, $\vec{p}_{\Lambda}$. The electron scattering angle is denoted by $\theta_{e}$. In the figure below $\theta_{\mathrm{K}}$ and $\theta_{\Lambda}$ are the reaction angles for kaon and $\Lambda$, respectively. Each is measured with respect to $\vec{q}$, the direction of momentum transfer.

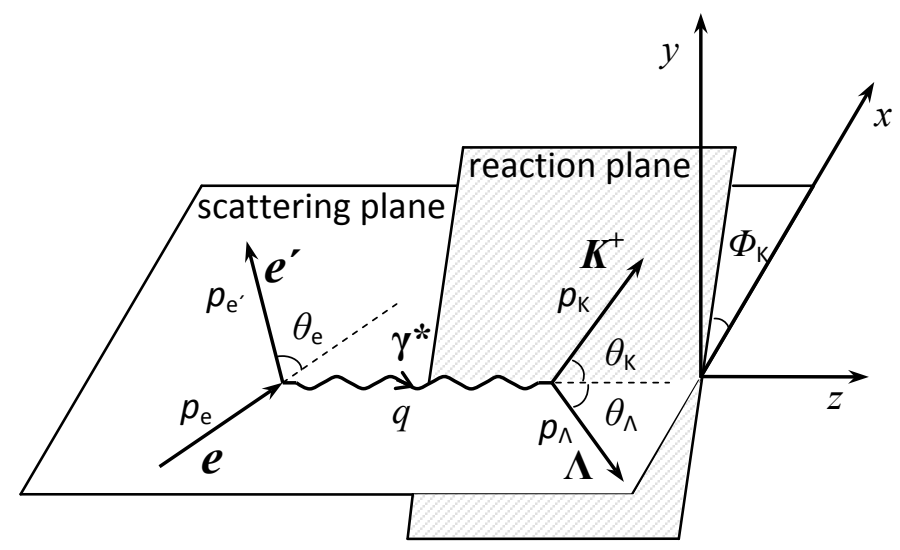

Figure 1.5.4 Kinematics of the hypernuclear electroproduction.

The electron projected on the target has a fairly high energy, which allows it to penetrate deep into the nucleus and interact with the proton. The interaction occurs via the exchange of a virtual photon turning the positive proton into neutral $\Lambda$. To preserve 
the electric charge and strangeness of the system a positive kaon $\left(K^{+}\right)$must be released in the reaction as well.

The four-momentum for each particle is written as

$$
\begin{array}{lr}
\text { incident electron }(e): & p_{e}=\left(E_{e}, \vec{p}_{e}\right), \\
\text { recoil electron }\left(e^{\prime}\right): & p_{e^{\prime}}=\left(E_{e^{\prime}}, \vec{p}_{e^{\prime}}\right), \\
\text { proton in target }(p): & p_{p}=\left(m_{p}, 0\right), \\
\text { virtual photon }(\gamma): & q=(\omega, \vec{q}), \\
\text { kaon }\left(K^{+}\right): & p_{K}=\left(E_{K}, \vec{p}_{K}\right), \\
\text { lambda }(\Lambda): & p_{\Lambda}=\left(E_{\Lambda}, \vec{p}_{\Lambda}\right) .
\end{array}
$$

Here the energy transfer from an incident electron to the virtual photon is $\omega=E_{e}-E_{e^{\prime}}$.

Cross sections can be calculated with the same formalism used for pion electroproduction on the nucleon [13; 14]. Thus, the expression for the triple-differential cross section can be written as [15]

$$
\begin{aligned}
\frac{d^{3} \sigma}{d_{e^{\prime}} d \Omega_{e^{\prime}} B \Omega_{K}} & =\Gamma\left[\frac{d \sigma_{T}}{d \Omega_{K}}+\varepsilon \frac{d \sigma_{L}}{d \Omega_{K}}+\varepsilon \frac{d \sigma_{P}}{d \Omega_{K}} \cos \left(2 \Phi_{K}\right)\right. \\
& \left.+\sqrt{2 \varepsilon(1+\varepsilon)} \frac{d \sigma_{I}}{d \Omega_{K}} \cos \left(\Phi_{K}\right)\right]
\end{aligned},
$$

where $\sigma_{T}, \sigma_{L}, \sigma_{P}$, and $\sigma_{I}$ are, respectively, called transverse, longitudinal, polarization and interference cross sections. Formula (1.5.10) is calculated in the onephoton exchange approximation in the CMS (center-of-mass) frame. The higher order diagrams have a negligible contribution since they should be smaller by a factor $\alpha=e^{2} / \hbar c=1 / 137$. 
The virtual photon flux factor $\Gamma$ in the laboratory frame takes the following form

$$
\Gamma=\frac{\alpha}{2 \pi^{2} Q^{2}} \frac{E_{\gamma}}{1-\varepsilon} \frac{E_{e^{\prime}}}{E_{e}}
$$

with the effective photon energy

$$
E_{\gamma}=\omega-q^{2} /\left(2 m_{p}\right)
$$

The invariant momentum transfer squared is defined as $Q^{2}=\vec{q}^{2}-\omega^{2}=4 E_{e} E_{e^{\prime}} \sin ^{2}\left(\theta_{e} / 2\right)$ when neglecting the mass of the electron. Here, by effective photon energy we understand the energy of the real photon which in the laboratory frame will yield the same invariant $s=\left(p_{\gamma}+p_{p}\right)$ as the virtual photon in the center of mass of the elementary electroproduction reaction.

In the equations above the polarization factor is

$$
\varepsilon=\left[1+\frac{2|q|^{2}}{Q^{2}} \tan ^{2}\left(\theta_{e} / 2\right)\right]^{-1}
$$

The explicit expressions for $\sigma_{T}, \sigma_{L}, \sigma_{P}$, and $\sigma_{I}$ in terms of the hadron tensor $W^{\mu \eta}$, for example, can be found in [15]. 


\subsubsection{Hypernuclear electroproduction process}

Because in the experiment there is an electron beam bombarding not a free proton, but a target of nuclear mass $m_{A}$, we may obtain the hyperon either free from the target nucleus or bound to it, depending on the momentum transfer to the hyperon. To study the properties of $\Lambda \mathrm{N}$ interaction we would be primarily interested in $\Lambda$ bound states, i.e., in the hypernucleus itself.

Now the theoretical framework used for the elementary process in section 1.5.3 has to be applied to the electroproduction of hypernuclei $(\mathrm{H})$ in the nuclear medium (with mass number $A$ ):

$$
e+\mathrm{A} \rightarrow e^{\prime}+K^{+}+\mathrm{H}
$$

Schematically the process is presented in Fig. 1.5.5.

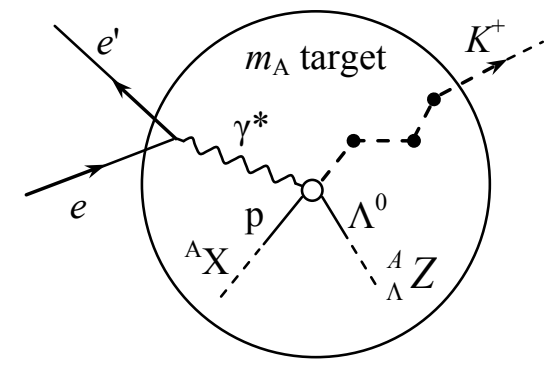

Figure 1.5.5 Schematic representation of ${ }^{A} X\left(e, e^{\prime} K^{+}\right){ }_{\Lambda}^{A} Z$ hypernuclear electroproduction.

The elementary process $\left(e, e^{\prime} K^{+}\right)$initiates the hadronic transition $p \rightarrow \Lambda^{0}$. The final products are the $K^{+}$and the bound $\Lambda$, which together with the affected nucleus forms the 
hypernucleus $\mathrm{H}\left({ }_{\Lambda}^{A} \mathrm{Z}\right)$. In comparison to the elementary process, the presence of the nuclear matter produces additional features [16]: the presence of the other nucleons might modify the elementary process; the outgoing kaon might scatter on its way out of the hypernucleus; the initial and final states now involve the many-body nuclear and hypernuclear wave functions.

The cross section of the process still can be described by the expression (1.5.10) with 4-momenta and the masses of the proton and the hyperon changed to those of the nucleus $\mathrm{A}$ and hypernucleus $\mathrm{H}$, respectively. The detailed explanations of the crosssection calculations for hypernuclear electroproduction are presented in works $[15,17]$.

Following the notations made in section 1.5.3, energy and momentum conservation laws lead to:

$$
\begin{gathered}
E_{e}-E_{e^{\prime}}+m_{A}=\omega+m_{A}=E_{K}+E_{H}, \\
\vec{p}_{e}-\vec{p}_{e^{\prime}}=\vec{q}=\vec{p}_{K}+\vec{p}_{H} .
\end{gathered}
$$

Solving for $\vec{p}_{H}$ in expression (1.5.14) and squaring the result we obtain

$$
p_{H}^{2}=p_{e}^{2}+p_{e^{\prime}}^{2}+p_{K}^{2}-2 \vec{p}_{e} \cdot \vec{p}_{e^{\prime}}-2 \vec{p}_{e} \cdot \vec{p}_{K}+2 \vec{p}_{e^{\prime}} \cdot \vec{p}_{K},
$$

or in terms of the angles $\theta_{e e^{\prime}}, \theta_{e K}$, and $\theta_{e^{\prime} K}$,

$$
p_{H}^{2}=p_{e}^{2}+p_{e^{\prime}}^{2}+p_{K}^{2}-2 p_{e} p_{e^{\prime}} \cos \left(\theta_{e e^{\prime}}\right)-2 p_{e} p_{K} \cos \left(\theta_{e K}\right)+2 p_{e^{\prime}} p_{K} \cos \left(\theta_{e^{\prime} K}\right) .
$$


The angles in the cross terms are calculated from the geometry of experiment. From (1.5.13) the square of the hypernucleus energy is

$$
\left.E_{H}^{2}=\left(E_{e}-E_{e^{\prime}}+m_{A}-E_{K}\right)^{2}=\left(\omega+m_{A}\right)^{2}+E_{K}^{2}-2 \omega+m_{A}\right) E_{K} .
$$

With results from Equations (1.5.16) and (1.5.17) the missing mass (hypernuclear mass) calculations are straightforward:

$$
m_{H}=\sqrt{E_{H}^{2}-p_{H}^{2}} .
$$

\subsection{Baryon-Baryon interactions}

As I mentioned earlier, the baryon-baryon interactions are still not well understood. We have a confident knowledge and understanding of nucleon-nucleon (NN) interactions, which over the last several decades have been supported with plenty of experimental data. With the advent of hypernuclei, new interaction classes evolved. Now, the baryonbaryon models had to include the hyperon-nucleon (YN) and hyperon-hyperon (YY) interactions, since the hyperon as well as a nucleon (proton or neutron) belong to a baryon group. A new nuclear structure that cannot be seen in an ordinary nucleus may be revealed in the presence of the embedded hyperon. Such information can provide valuable input in the flavor SU(3) symmetry and finally help to build a unified baryonbaryon interaction theory. However, short lifetimes of hyperons and the fact that the free hyperons were mostly produced from a secondary beam, created great limitations on experimental studies of YN interactions. Therefore, the YN data is very limited in 
quantity and precision. The hypernuclear electroproduction experiments bring a fresh breath into the theory of YN interactions. Namely, hypernuclear spectroscopy plays a key role in the investigation of $\mathrm{YN}$ interactions since it allows probing the ground and excited states of the nucleus core with the help of an implanted hyperon.

The hyperon interaction with all the nucleons inside of the hypernucleus has to be presented as a nuclear many-body problem. Since the forces between the baryons are hadronic with the time scales comparable to the lifetime of the $\Lambda$, the hypernuclear system may utilize the well developed nuclear theory of strong interactions. The hypernucleus can be viewed as a conglomerate of baryons, where each baryon interacts with an effective potential formed by the rest. The hypernuclear Hamiltonian can be expressed as [18]

$$
H=H_{\text {core }}+T_{\mathrm{Y}}+\sum v_{\mathrm{YN}}^{\text {effective }}
$$

The Hamiltonian $H_{\text {core }}$ describes the core nucleus, $T_{\mathrm{Y}}$ represents the kinetic energy of the hyperon and $v_{\Lambda \mathrm{N}}^{\text {effective }}$ is responsible for the effective $\mathrm{YN}$ interaction.

The Hamiltonian of the core nucleus is well reproduced by the Cohen-Kurath shell model [19]. The effective YN potential is usually constructed via a G-matrix that, to first approximation, is calculated in a free-space two-body approach. These two-body interactions are commonly described by One-Boson-Exchange (OBE) models such as Nijmegen $[20 ; 21 ; 22]$ and Julich $[23 ; 24]$, which are based on the extension of NN interaction models (meson-exchange models) on the broken flavor SU(3) symmetry. Frequently the effective potential is written in the form of a three-range Gaussian [25]: 
$V_{\Lambda \mathrm{N}}=\sum_{i=1}^{3}\left(a_{i}+b_{i} k_{f}+c_{i} k_{f}^{2}\right) \exp \left(-r^{2} / \beta_{i}^{2}\right)$. This representation allows calculating the level structure and reaction cross sections, which agree reasonably well with experimental data for light hypernuclear systems, $A \leq 5$.

The other phenomenological approach to the effective $\Lambda \mathrm{N}$ interaction that includes the heavier, $p$-shell hypernuclei is written in the form

$$
V_{\Lambda \mathrm{N}}(r)=V_{0}(r)+V_{S}(r) \cdot S_{\Lambda} \cdot S_{N}+V_{L S}\left(L \times S^{+}\right)+V_{A L S}\left(L \times S^{-}\right)+V_{T} \cdot S_{12}
$$

In the above equation, $V_{0}(r)$ is the average central interaction potential, $V_{S}(r)$ is the spin-spin interaction potential, $V_{L S}$ and $V_{A L S}$ are, respectively, the symmetrical and asymmetrical spin-orbit interaction potentials, and finally $V_{T}$ is the tensor interaction term. The $S_{12}=3\left(\sigma_{1} \cdot \hat{r}\right)\left(\sigma_{2} \cdot \hat{r}\right)-\sigma_{1} \cdot \sigma_{2}$ is the spin-tensor operator and $S^{ \pm}=1 / 2\left(S_{\mathrm{N}} \pm S_{\mathrm{Y}}\right)$ are the symmetric and anti-symmetric combinations of nucleon and hyperon spin operators. In the shell model representation, the $p$-shell hypernuclei with a $\Lambda$ in the 0 s orbit can be described by five radial integrals for $s_{\Lambda} p_{\mathrm{N}}$ wave function. The spin-dependent terms are denoted as $\Delta, S_{\Lambda}, S_{\mathrm{N}}$, and $T$, respectively, for the spin-spin term, the $\Lambda$ spin dependent spin-orbit term, the $\mathrm{N}$ spin dependent spin-orbit term, and the tensor term. The central potential term in the literature is denoted as $\bar{V}[9,26,27]$. These parameters are mostly derived from the spacing of the doublets and excitation energies of $p$-shell $\Lambda$ hypernuclear data and then compared with theoretical predictions based on Gmatrix calculations of the free $\Lambda \mathrm{N}$ interactions. 


\section{$1.7 \quad s$ and $p$ shells in $\Lambda$ hypernuclei}

The following hypernuclei, ${ }_{\Lambda}^{3} \mathrm{H},{ }_{\Lambda}^{4} \mathrm{H},{ }_{\Lambda}^{4} \mathrm{He}$, and ${ }_{\Lambda}^{5} \mathrm{He}$ belong to the $s$-shell hypernuclei. They are composed by adding a $\Lambda$ hyperon to the corresponding core nuclei, ${ }^{2} \mathrm{H},{ }^{3} \mathrm{H},{ }^{3} \mathrm{He}$, and ${ }^{4} \mathrm{He}$. All of them contain a relatively small number of baryons, ranging from three to five, which makes these hypernuclei to be few-body systems. Since the number of baryons is not high, these nuclear systems are convenient for calculations.

The $A=4$ hypernuclear system, such as ${ }_{\Lambda}^{4} \mathrm{H}$ and ${ }_{\Lambda}^{4} \mathrm{He}$, provide the most accurate information on the spin-spin part of the $\Lambda \mathrm{N}$ interaction [28]. In the ground state ( $s$-shell) all four particles are coupled to $J^{p}=0^{+}$state, resembling in such configuration an alpha particle. However, the $\Lambda$ is distinguishable from the nucleons, which allows the system to also couple to an excited $J^{p}=1^{+}$state. This is achieved by flipping the spin of the $\Lambda$. The analysis of singlet and triplet scattering lengths $a_{s}$ and $a_{t}$, and comparison of their ratio to $\Lambda p$ scattering data ( $a_{s} / a_{t} \approx 1$ ), allows adjusting the models for the spin-spin potential term. The ratio $a_{s} / a_{t} \approx 4$ was determined from the analysis of double pion exchange in $\Lambda \Sigma$ coupling and suggested a strong spin dependence in the $\Lambda \mathrm{N}$ interactions [29]. A detailed theoretical analysis of $s$-shell hypernuclei can be found, for example, in works [30] and [31].

All the rest of the so-far identified hypernuclei correspond to nuclei with nucleons in the $p$-shell and beyond. Currently, there are more experimental data for $p$-shell hypernuclei then for $s$-shell. However, the quality of the $p$-shell data is not as good as for the $s$-shell data. The hypernuclear electroproduction experiments that have been recently 
carried out at Jefferson Laboratory, HNSS and HKS, bring a new generation in high resolution spectroscopy of medium-to-heavy hypernuclear systems.

The calculation of the $p$-shell hypernuclear structure, as well as the standard nuclear system, is a challenging task because of the high number of interacting particles. To simplify the process, specific assumptions about the structure of the nucleus carrier are made. There are mainly two approaches dominating the theory of $p$-shell hypernuclei: 1) clustering of the core nucleus, 2) free $\Lambda$ hyperon in a self-consistent field of nucleons. In the first method, the core nucleus is considered as a combination of several particles and/or smaller nuclear clusters $[32 ; 33]$. For example, ${ }_{\Lambda}^{9} \mathrm{Be}$ is described as two alpha particles and a $\Lambda$ hyperon $(2 \alpha+\Lambda),{ }_{\Lambda}^{13} \mathrm{C}$ as $(3 \alpha+\Lambda),{ }_{\Lambda}^{6} \mathrm{He}$ as $(\alpha+n+\Lambda)$, and ${ }_{\Lambda}^{7} \mathrm{Li}$ as $(\alpha$ $+d+\Lambda$ ) [29]. In such a way the complexity of the multi-body calculations is greatly reduced. During the analysis, the interaction potentials of a $\Lambda$ with nuclear clusters are matched up with the known potentials of the light hypernuclei. In the second method, the $\Lambda$ is interacting with a self-consistent field of nucleons, which is composed of the $\Lambda \mathrm{N}$ potentials and the density of nucleons [34]: $V\left(\boldsymbol{r}_{\Lambda}\right)=\int \rho_{N}(\boldsymbol{r}) V_{\Lambda N}\left(\left|\boldsymbol{r}_{\Lambda}-\boldsymbol{r}\right|\right) d \boldsymbol{r}$. Here the $\rho_{N}(\boldsymbol{r})$ is the density of the nucleons, and $V_{\Lambda N}$ is the averaged, spin-isospin independent $\Lambda \mathrm{N}$ potential.

The relatively new trend in describing the $p$-shell hypernuclear system is based on the shell model calculations with Hamiltonians of the form of (1.6.1) and the effective $\Lambda \mathrm{N}$ potential in the form (1.6.2). As we already mentioned in the previous Section 1.6, the calculations for the $p$-shell are generally made with a phenomenological interaction fit 
to experimental $p$-shell data. The detailed formalism of shell-model calculations is described in the works $[18,35]$.

\subsection{Significance of research and recent experimental data}

As it was already stated in the previous paragraphs, hypernuclear spectroscopy studies the properties of the hypernuclear states that can improve our understanding of the baryon-baryon interaction, which for its completeness requires the information on the hyperon-nucleon and hyperon-hyperon interactions. The research in the field of hypernuclear physics might bring a better understanding of the charge symmetry breaking (CSB) mechanism, help to investigate the three body forces (3BF), allow precise lifetime measurements at different masses via non-mesonic weak decay, explain the presence of free strange particles in neutron stars, and, most importantly, build a unified theory of baryon-baryon interactions. Currently the research gravitates toward finding the appropriate $\Lambda \mathrm{N}$ interaction potentials that could explain the structural distribution of the states inside of hypernuclei. The internal excited states are important for shell model calculations. The knowledge of spin-orbit splitting is the key to understanding the spin-dependent part of the $\Lambda \mathrm{N}$ potential. It was found that the intrinsic width of the states is very narrow, $\approx 100 \mathrm{keV}$. To succeed in such a study, high resolution spectroscopy is highly desirable. The best energy resolution (until the year 2000) in reaction spectroscopy using mesonic beams $\left(K^{-}\right.$or $\left.\pi^{+}\right)$was reported to be $1.45-2.0 \mathrm{MeV}$ (FWHM). The experiments that reported such values were conducted at KEK using the 
Superconducting Kaon Spectrometer (SKS) and at the BNL Alternating-Gradient Synchrotron (AGS) (Fig. 1.8.1).
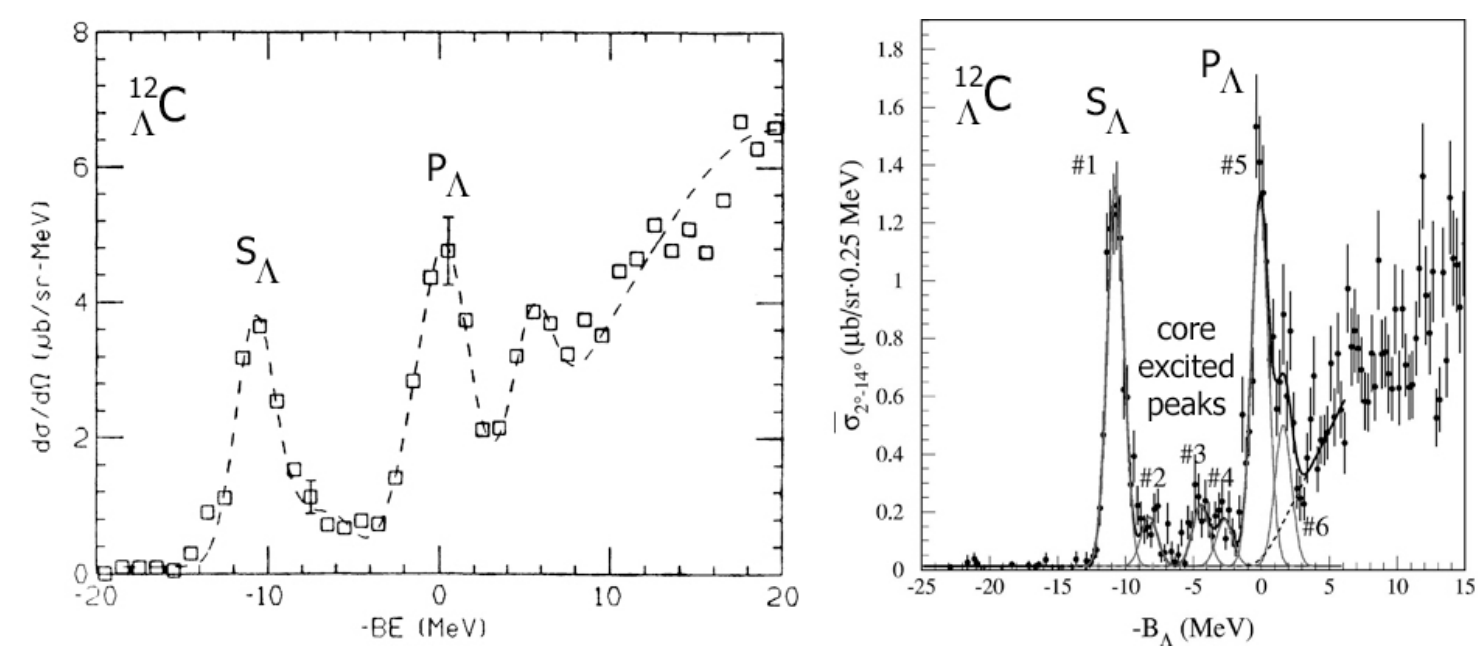

Figure 1.8.1 Hypernuclear binding energy spectrum of ${ }_{\Lambda}^{12} \mathrm{C}$ from BNL (left [36]) and KEK (right [38]) produced by $\left(\pi^{+}, K^{+}\right)$reaction.

In the figure above, the missing mass spectra of ${ }_{\Lambda}^{12} \mathrm{C}$ obtained via $\left(\pi^{+}, K^{+}\right)$reaction at BNL and KEK are shown. There is a clear indication of the better energy resolution of the ground state peaks with a $\Lambda$ in $s$ and $p$ shells in the KEK data. The poor resolution of the BNL data (on the left) does not allow one to see the core-excited states that are visible in the KEK spectrum, (peaks \#2, \#3 and \#4). The comparison of BNL and KEK data demonstrates the importance of good energy resolution in the spectra. Despite great progress in the peaks' resolution, there was a lot of controversy in the understanding of spin-orbit splitting. Some of the BNL data (on carbon and beryllium targets) suggested a very small spin-orbit splitting and therefore a small strength in the spin-orbit force, [36]. 
However, the KEK analysis of ${ }_{\Lambda}^{16} \mathrm{O}$ offered larger numbers for $p$-shell $(0.3-0.6 \mathrm{MeV}$ [37]) and $f$-shell (1.7 MeV [38]).

The high quality, high intensity CW electron beam available in the Jefferson Lab, permitted production of the first hypernuclear spectra via the $\left(e, e^{\prime} K^{+}\right)$reaction. The first experiment E89-009 (HNSS), completed in 2000 at Jefferson Lab (Hall C), proved the feasibility of using electromagnetic probes and the associated technique, and obtained high resolution spectroscopy of ${ }_{\Lambda}^{12} \mathrm{~B}$. Figure 1.8.2 shows the measured spectrum with the background (shaded area) and theoretical calculations overlaid on the data (curve). The achieved energy resolution of $\approx 700 \mathrm{keV}$ set the record in reaction spectroscopy (Fig. 1.8.2) [12].

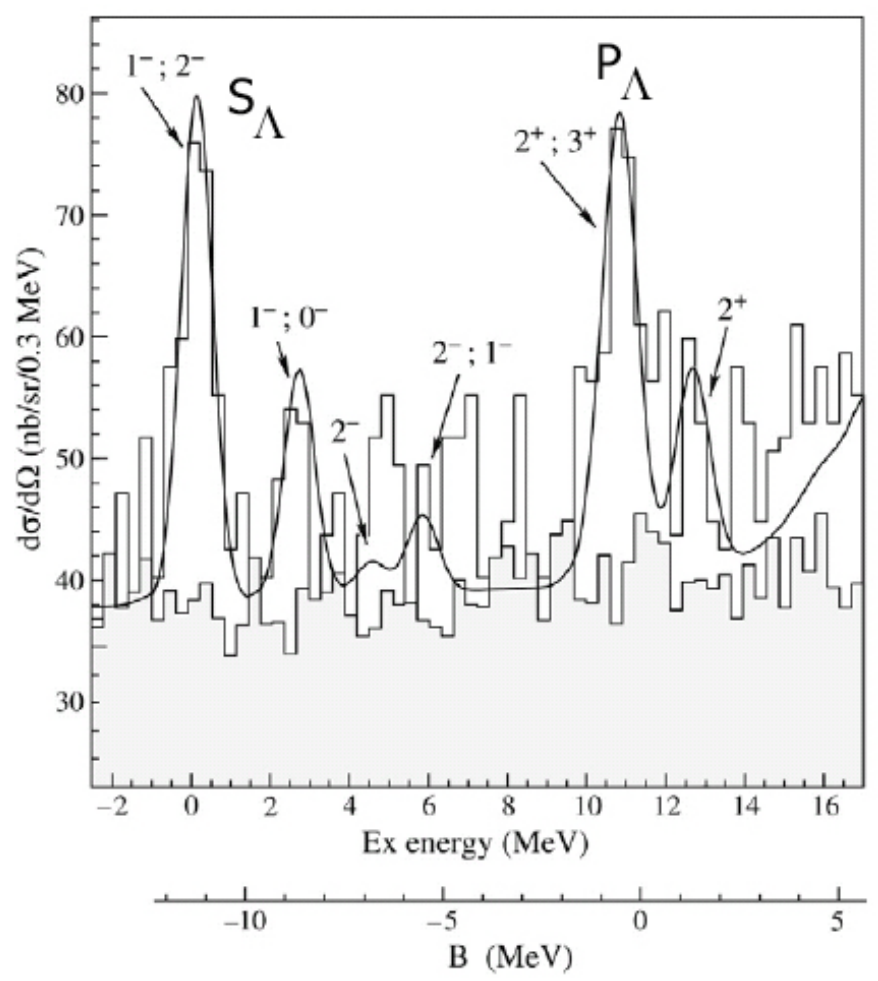

Figure 1.8.2 The HNSS hypernuclear spectrum of ${ }_{\Lambda}^{12} \mathrm{~B}$ [12]. The description of the plot is given in text. 
The experiment showed the feasibility of hypernuclear studies with electron beams. The E01-011 (HKS) experiment described in this dissertation is the next step in the hypernuclear program at Jefferson Laboratory. Application of the new high resolution spectrometers instead of the standard Hall C spectrometers, and introduction of new experimental techniques, such as the "tilt method", allowed achieving the energy spectra of light-medium hypernuclei with improved energy resolution.

\subsection{Dissertation Objectives}

In this dissertation the analysis of the experimental data collected during E01-011 (HKS) experiment in Fall 2005 at Thomas Jefferson National Accelerator Facility will be presented. The study of the experimentally obtained, high-resolution excitation spectra will help test the theoretical predictions for $\Lambda \mathrm{N}$ interactions. The main emphasis of the work will be given to the physics of the core-excited states. The appearance of such states in the excitation spectra with significant statistics is a challenging task that requires rigorous data analysis. The novel approach to particle identification based on statistical treatment of the particle detectors will be introduced. This method, called the likelihood method, will serve as a substitute to the commonly used approach that involves the hardcut technique. As a result of the application of hard cuts, the spectrum might lose events of interest, in our case kaons, because of the limiting nature of the cuts. In other words by placing the limiting cut on the kaon distribution that has a strong overlap with the distribution of other particles, I would not count the kaons present in the chopped tails. It is worth mentioning that particle identification usually requires the use of signals from several different detectors, where the strong overlaps of the particles are always present. 
If the likelihood approach proves to be efficient, we expect to significantly improve the statistics of the spectra. Efficient kaon particle identification (PID) is key to obtaining good signal-to-background ratios. The goal is to improve statistical significance, especially for low statistic core excited states observed in the excitation spectra. The precision of extracting the peaks location in the missing-mass spectra together with their statistical significance are important when comparing to theoretical predictions.

The dissertation aims to analyze the ${ }_{\Lambda}^{12} \mathrm{~B},{ }_{\Lambda}^{7} \mathrm{He}$, and ${ }_{\Lambda}^{28} \mathrm{Al}$ hypernuclei. The interpretation of the physics for each spectrum will be presented with a special emphasis on the core-excited states configurations. 


\section{CHAPTER 2}

\section{EXPERIMENTAL SETUP AND TECHNIQUES}

\subsection{Overview}

The experiment E01-011 [39], "Spectroscopic study of $\Lambda$ hypernuclei up to mediumheavy mass region through the $\left(e, e^{\prime} K^{+}\right)$reaction," was performed at the Thomas Jefferson National Accelerator Facility (JLab) [40] from June to October 2005. The main goal of the experiment was to obtain $\Lambda$ hypernuclei spectra with high energy resolution reaching a few hundreds of $\mathrm{keV} \Leftarrow 300-400 \mathrm{keV}$ ). As a result of the unique characteristics of the reaction, the spin states with both spin-flip and spin-non-flip amplitudes were populated. The spin-stretched states refer to particle-hole configurations where both the particle and the hole are in the lowest $j=l+1 / 2$ subshells, and are coupled to the maximum possible angular momentum $l_{\mathrm{p}}+l_{\mathrm{h}}$. Exotic neutron-rich and mirror (to previously studied) hypernuclei were obtained with high statistics. The use of medium and heavy targets allowed observing $\Lambda$ s bound in $s$-shell, $p$-shell, and beyond.

\subsection{Experimental facility (Jefferson Laboratory)}

The experiment took full advantage of the high quality electron beam at the JLab accelerator facility. The laboratory's superconducting radio frequency (SRF) Continuous Electron Beam Accelerator Facility (CEBAF) simultaneously delivers high energy (up to 
$6 \mathrm{GeV})$ continuous wave $(\mathrm{CW})$ beams to the three experimental halls: Hall A, Hall B, and Hall C [40].

CEBAF consists of the injector system, two straight linac branches (North and South), two recirculation arcs (East and West), the Beam Switchyard (BSY), and three experimental halls with the corresponding end stations (Fig. 2.2.1). The Jefferson Lab also includes the $10 \mathrm{~kW}$ Free Electron Laser (FEL) located in the center of CEBAF. An aerial view of the facility is shown in Fig. 2.2.2.

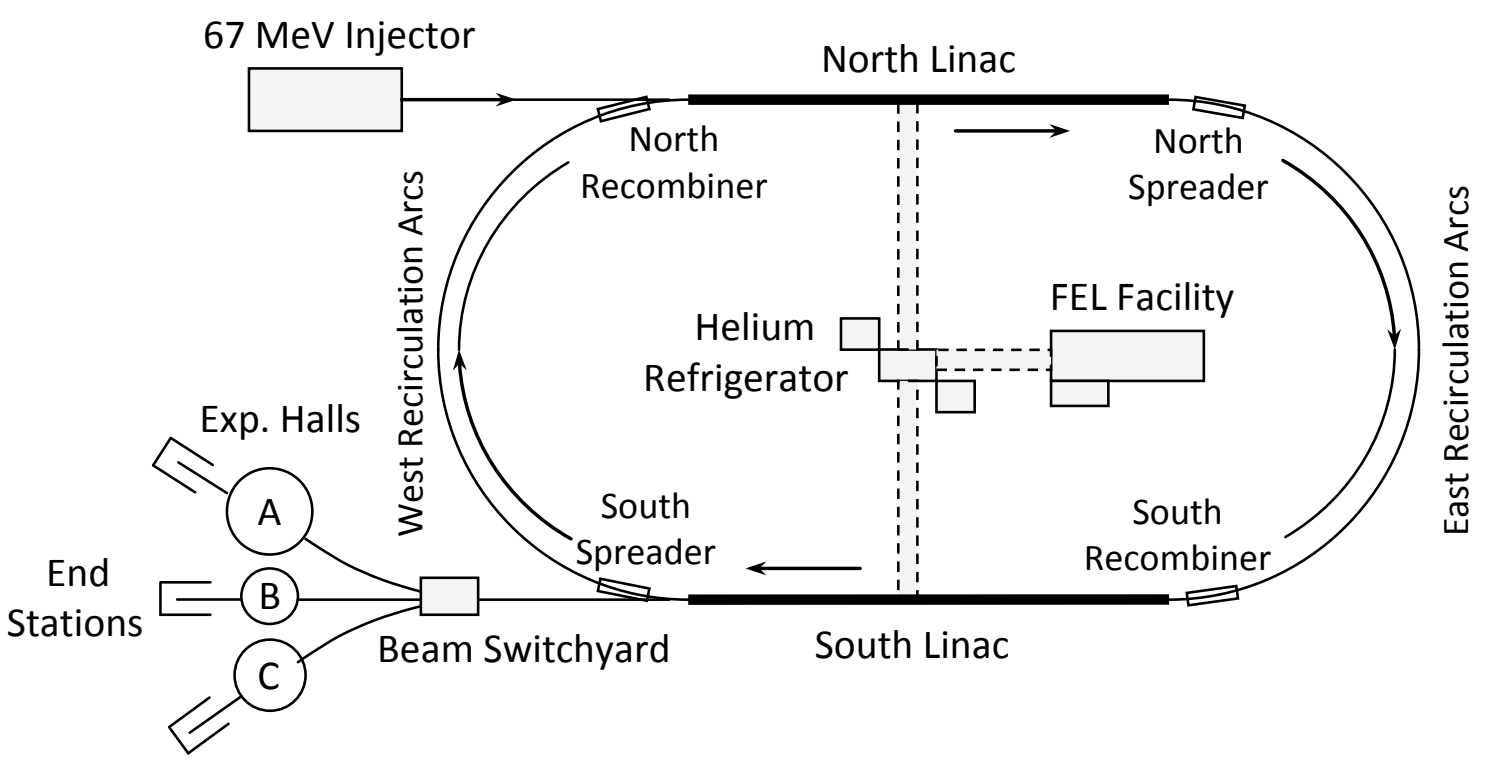

Figure 2.2.1 Schematic top view of CEBAF accelerator. The arrows indicate the direction of the electron beam. 


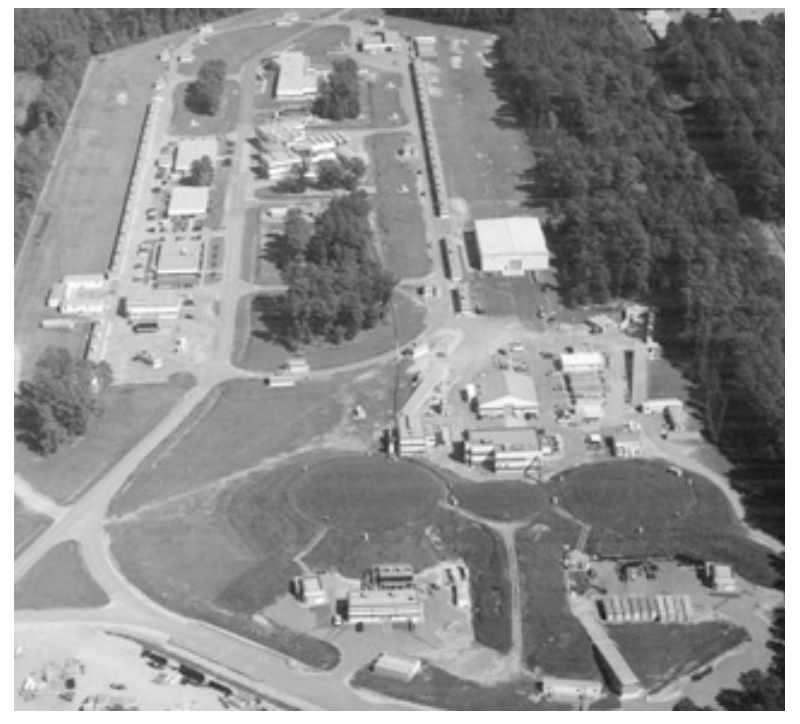

Figure 2.2.2 Aerial view of the CEBAF accelerator complex. The shape of the underground accelerator is suggested by the structure of the service buildings along the track [40].

\subsubsection{Injector system}

The $67 \mathrm{MeV}$ injector system acquires its electron beam from a gallium arsenide (GaAs) photocathode gun. Three independent radio frequency (RF) gain-switched, circularlypolarized lasers are directed at the single photocathode. They produce the three interlaced $499 \mathrm{MHz}$ electron beams which are accelerated in an electrostatic field to $100 \mathrm{keV}$. Spaced apart by $120^{\circ}$ of RF phase, they result in $1497 \mathrm{MHz}, 55$ ps bunched beam. Each of the separated bunches can be produced with unique properties, which are repeated every third bunch. Then the beam is longitudinally compressed to 2 ps and accelerated from $100 \mathrm{keV}$ to $67 \mathrm{MeV}$ by 18 superconducting cavities. 


\subsubsection{Linacs and recirculation ares}

The North and South Linacs are the two longest, antiparallel, straight sections of the CEBAF. The beam exiting the injector with $67 \mathrm{MeV}$ energy is forwarded to the North Linac, where $160 \mathrm{SRF}$ niobium cavities accelerate it to $600 \mathrm{MeV}$. In order to function properly, the cavities are bathed in $2 \mathrm{~K}$ liquid helium contained within cryomodules. The liquid helium is supplied by the Central Helium Liquefier located at the center of the accelerator facility. In total each linac is made up of 20 cryomodules, with 8 accelerating cavities inside of each module. The 180 degree bending of the beam is done in the East Arc. After passing through the 80-meter-radius arc, the beam is directed into the South Linac, where it gains another $600 \mathrm{MeV}$ energy boost. After passing the South Linac the electron beam can be extracted to the experimental halls or sent through the West Recirculation Arc into the North Linac for further acceleration. To achieve maximum energy, $6 \mathrm{GeV}$, the electrons have to pass the whole track five times gaining at each turn 1.2 GeV. Since the beam can be recirculated five times, there must be five different settings of the bending magnetic field. This is achieved by having five (on the East) and four (on the West) separate arcs of the same radius with different magnetic settings. In order to properly steer the beam into the recirculation arcs and back into the linac, vertical magnetic spreader and recombiner systems are installed at each end of both arcs as shown in Fig. 2.2.1. The experimental halls can simultaneously receive beam at different energies. It is also possible to run all three halls simultaneously at the maximum energy. 


\subsubsection{Beam switchyard}

The Beam Switchyard located at the end of the South Linac contains sophisticated machinery for delivering the electron beam into the three experimental Halls. The BSY employs the BSY extractor to distribute the beam among the three halls and BSY recombiner to combine the electrons into a single pile heading further down the passageway and directed to one of the end stations. The BSY Extractor employs 499 $\mathrm{MHz}$ RF extraction modules, similar to cavities, which kick out every third bunch of the $1497 \mathrm{MHz}$ beam to each experimental hall.

\subsubsection{CEBAF beam properties}

The CEBAF produces a beam structure of 2 ps long bunches in every 2 ns time interval. At such a rate the beam is considered to be a quasi-continuous wave $(\mathrm{CW})$. The continuity of the beam is an important requirement for coincidence experiments, where the rate of accidentals is inversely proportional to the beam duty factor. The CEBAF has almost $100 \%$ duty factor providing the experimental halls with currents up to $200 \mu \mathrm{A}$. It is capable of supplying Halls $\mathrm{A}$ and $\mathrm{C}$ with beams of high polarization and high current (10's of $\mathrm{nA}$ ), while maintaining a high polarization and low current beam delivery into Hall B. The electron beam produced in CEBAF can be more than $75 \%$ polarized or unpolarized, depending on the requirements of the experiments. The beam polarization is achieved in the injector system, by radiating the GaAs photocathode with circularly polarized photons. The lowest operating energy of CEBAF is $0.6 \mathrm{GeV}$, while at the present time the beam can reach a maximum energy of $6 \mathrm{GeV}$. The upgrade of the 
CEBAF facility to $12 \mathrm{GeV}$ is an exciting upcoming event. Here, we will skip the details of this upgrade, however they can be found in reference [40].

The principal parameters of the CEBAF electron beam are taken from reference [40] and summarized in Table 2.2.1.

Table 2.2.1 Principal CEBAF electron beam parameters [40].

\begin{tabular}{llll}
\hline Beam energy & $\approx 6 \mathrm{GeV}$ & Geometrical emmitance & $\approx 10^{-9} \mathrm{~m} \cdot \mathrm{rad}$ \\
Relative energy spread & $2.5 \cdot 10^{-5}$ & Relative momentum spread & few $10^{-5}$ \\
Beam polarization & $>75 \%$ & Average current (Halls A \& C) & $1-150 \mu \mathrm{A}$ \\
Beam transverse size & $\approx 80 \mu \mathrm{m}$ & Average current (Hall B) & $1-100 \mathrm{nA}$ \\
Bunch length & $300 \mathrm{fs}, 90 \mu \mathrm{m}$ & Bunch charge & $<0.3 \mathrm{pC}$ \\
Fundamental frequency & $1470 \mathrm{MHz}$ & Beam power & $<1 \mathrm{MW}$ \\
Beam frequency per Hall & $499 \mathrm{MHz}$ & Beam loss & $<1 \mu \mathrm{A}$ \\
\hline
\end{tabular}

\subsubsection{Hall $\mathrm{C}$ arc and beamline monitoring equipment}

After splitting in the Beam Switchyard, the electrons are directed into the experimental halls. Since our experiment was performed in Hall C, we will give a brief description of the Hall $\mathrm{C}$ beamline and supplementary equipment used for monitoring the beam. The beamline in general consists of several magnet systems ( 8 dipoles, 12 quadrupoles, 8 sextupoles) inside of the hall for steering the beam, and the beam diagnostic systems for monitoring and controlling the quality of the beam. The monitoring systems distributed through the beamline measure the beam positions, profile of the beam, its energy and current. The Hall $\mathrm{C}$ beamline layout is presented in Fig. 2.2.3. 


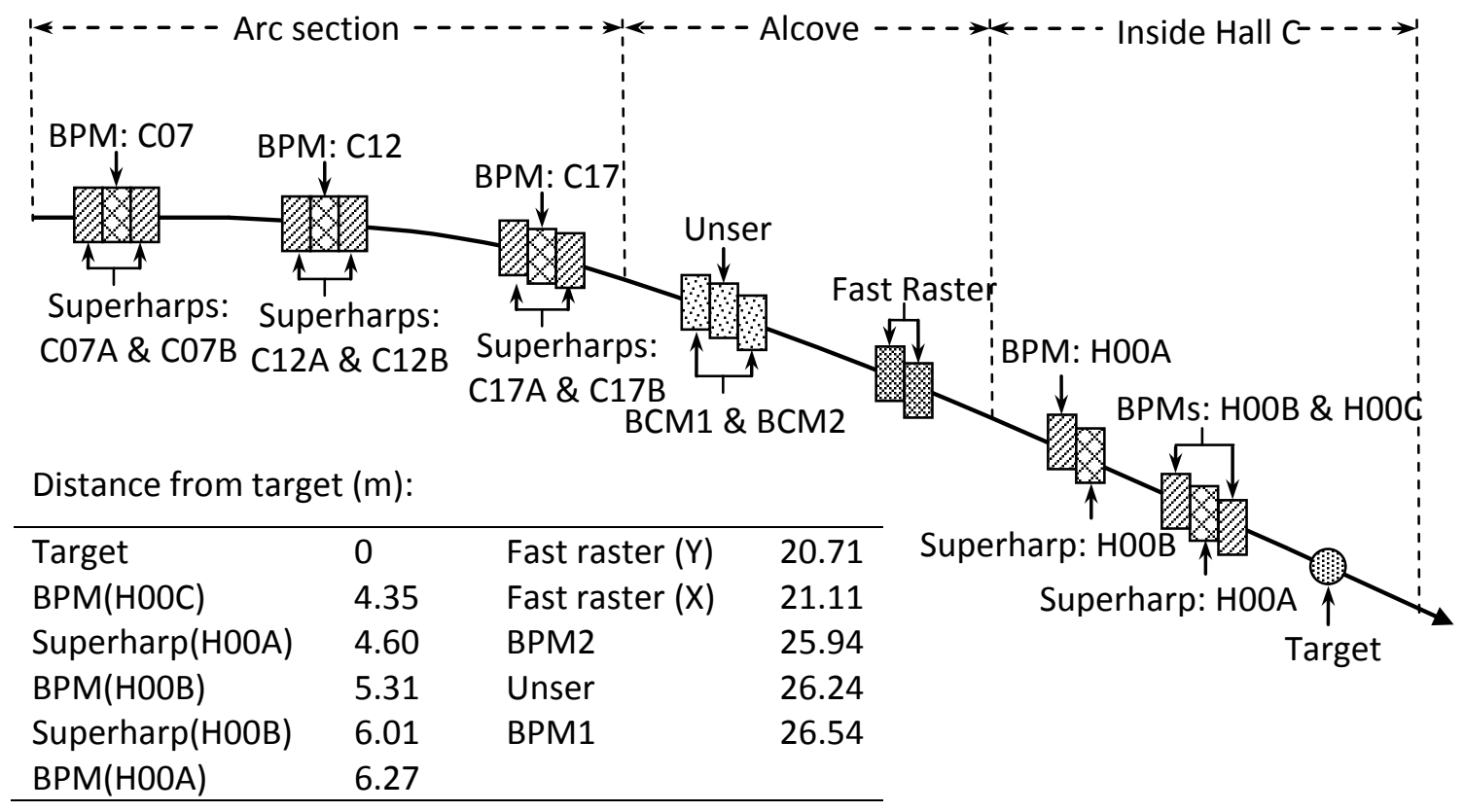

Figure 2.2.3 Schematics of Hall C beamline.

The beam exiting the BSY section enters the Hall C Arc area, then passes through the alcove of the hall and is directed towards the target. After the interaction with the target, the beam is transported out to the beam dump, while the reaction products are measured by the sets of the spectrometers and accompanying detectors. The geometrical shape of Hall $\mathrm{C}$ is circular with a diameter of $32 \mathrm{~m}$. The standard Hall $\mathrm{C}$ apparatus is comprised of two magnetic focusing spectrometers, the high momentum spectrometer (HMS) and short orbit spectrometer (SOS). The HMS serves to identify stable or longlifetime particles with momenta up to $7.5 \mathrm{GeV} / \mathrm{c}$, while the SOS allows for detection of short-lifetime particles, which tend to disintegrate within a short flight path. Some of the parameters of the spectrometers are shown in the Table 2.2.2. 
Table 2.2.2 Principal parameters of HMS and SOS.

\begin{tabular}{lll}
\hline Spectrometer & HMS & $\mathrm{SOS}$ \\
Maximum central momentum $\left(p_{0}\right)$ & $7.5 \mathrm{GeV} / \mathrm{c}$ & $1.8 \mathrm{GeV} / \mathrm{c}$ \\
Momentum range & $0.5-7.5 \mathrm{GeV} / \mathrm{c}$ & $0.1-1.8 \mathrm{GeV} / \mathrm{c}$ \\
Momentum resolution $\left(\Delta p / p_{0}\right)$ & $1 \times 10^{-3}$ & $1 \times 10^{-3}$ \\
Momentum bite $\left(p_{\max }-p_{\min }\right) / p_{0}$ & $18 \%$ & $40 \%$ \\
Bending mode & vertical & vertical \\
Angular range & $12.5^{\circ}-90^{\circ}$ & $14.5^{\circ}-168.4^{\circ}$ \\
Path length & $26 \mathrm{~m}$ & $10 \mathrm{~m}$ \\
Identified particles & long lifetime & short lifetime \\
\hline
\end{tabular}

Because of the specific kinematical requirements of the E01-011 experiment, such as a forward scattering angle and high momentum resolution, both spectrometers could not be used. Instead, two other spectrometers, HKS and ENGE, were successfully utilized. The parameters and description of the detector packages of these spectrometers will be presented later.

The beam diagnostics system consists of the Beam Position Monitors (BPM), the Beam Current Monitors (BCM), the Unser, the Beam Profile Monitors (superharps), and the Synchrotron Light Interferometer (SLI). These instruments allow instant monitoring of the beam quality throughout the experiment.

The BPM measures the beam position in the beamline. During the E01-011 experiment six BPMs were utilized (three in the Hall C Arc section and three in the area of the hall). The monitors consist of resonating cavities with a fundamental frequency matching both the accelerator, $1497 \mathrm{MHz}$, and Hall C, $499 \mathrm{MHz}$, frequencies. Each monitor is equipped with four antennas, positioned at $45^{\circ}$ with respect to horizontal and vertical axes. The strength of the signal acquired by each individual antenna allows determination of the relative position of the beam. The BPMs installed in the Hall C Arc 
area are used for beam transporting, while the BPMs located in Hall C are used for monitoring the position of the beam on the target.

The current of the electron beam is measured with the help of the two BCMs installed in the alcove of the hall. The BCM is a cylindrically-shaped, RF resonant cavity with the axis positioned along the beam. The cavity is tuned to excite the Transverse Electromagnetic $\left(\mathrm{TEM}_{010}\right)$ mode at the CEBAF beam frequency $1497 \mathrm{MHz}$. In the $\mathrm{TEM}_{010}$ mode the amplitude of the RF radiation is almost independent of the relative beam position. The output voltages of the wave guides are proportional to the beam current. Because the resonant cavities are sensitive to temperature change, the BCMs are thermally stabilized. To calibrate the gains and offsets of BCMs a parametric toroidal DC current transformer (Unser) is used. With good gain stability the Unser monitor allows measuring the absolute current. The BCM cavities and Unser monitor are enclosed in a box to improve magnetic shielding and temperature stabilization. Since the working principles of the $\mathrm{BCM}$ are based on electromagnetic interactions, the monitor is non destructive with respect to the quality of the beam. It is used all the time during the experiment without interrupting the beam.

The beam profile is measured with a device called superharp. The harp system consists of a wooden fork with three $22 \mu \mathrm{m}$ tungsten wires: two in the horizontal direction and one in the vertical direction. The fork can be inserted into the beamline at an angle of $45^{\circ}$ and thus moving both horizontal and vertical wires across the beam profile. The interaction of the electrons with the wires is recorded as a function of the harp position thus revealing the two dimensional beam profile. As a result of a variety of factors, for example uncertainty of the wire size, wire vibration, wire deformation, and 
mechanical and survey errors, the precision of the measurement is limited to about \pm 50 $\mu \mathrm{m}$ in each direction. The superharp is actually an update of the harp system with more sophisticated readout electronics and anti-vibration support mechanism [41]. During a harp scan, the interacting wires spread the beam profile, revealing the destructive nature of the scan. For this reason, the harp scans are only done occasionally to calibrate the beam between the run periods.

The energy of the beam in Hall $\mathrm{C}$ is measured by the magnet systems of the arc [42]. The method utilizes the principle of bending moving charged particles (electrons) in the magnetic field. The system of the magnets, mentioned earlier, bend the electrons in the Hall $\mathrm{C}$ Arc by a total of $\theta_{\text {arc }}=34.3^{\circ}$ along the $41.6 \mathrm{~m}$ transport line. Treating the steering magnets as spectrometers and accurately measuring the beam position with superharps, one can uniquely identify the momentum and therefore the energy of the electrons as

$$
E \approx p=\frac{e}{\theta_{\text {arc }}} \int B d l
$$

Here $e$ is the electron charge and the integration of the magnetic field $B$ is done over the path $l$ of the beam [43]. The magnetic field is meticulously mapped as a function of current. The actual mapping is done for the first dipole magnet, while the rest of the magnets are calibrated relative to the reference dipole. The reported precision of such a measurement is $\Delta E / E=0.5 \cdot 10^{-4}$ [42]. Since the method uses beam-destructive 
superharps and requires the focusing magnets to be switched off, the energy measurement cannot be done simultaneously with the data acquisition.

The continuous measurement of the beam energy spread is performed by the noninvasive Synchrotron Light Interferometer (SLI) [44]. This monitor utilizes the synchrotron light generated by the electron beam passing through a dipole with a 40meter bending radius. The synchrotron light is extracted through a quartz window by a mirror installed $12 \mathrm{~mm}$ away from the beam [45]. Then, with the help of $45^{\circ}$ mirrors, the light is guided toward a set of optical instruments. The setup is composed of a polarization filter, band pass filter, double slit assembly, focusing convex lens, and CCD camera [46]. The synchrotron light filtered into the polarized quasi-monochromatic wave passes through a double slit screen and creates an interference image focused on the CCD camera. The image from the CCD camera is recorded by computer and analyzed by specialized software. From the properties of the interference image, the RMS value of the beam size can be extracted and the energy spread calculated [46].

As we noted before, the transverse size of the electron beam is very small $(\approx q 0 \mathrm{~m}) \quad$, Table 2.2.1. Because of such a small size and the high power of the beam, there is a high probability that the beam will cause damage to the targets and the beam dump. In order to prevent that, two separate rastering systems, "fast" and "slow", are installed in the beamline. The "fast" rastering system is located 25 meters upstream of the target, while the "slow" one is positioned just before the scattering chamber in the beamline. The main purpose of the rastering systems is a reduction of the power-per-area delivered to the target and the dump. 
The "fast" rastering system is used for target protection. It rasters the beam over a $2 \times 2 \mathrm{~mm}^{2}$ area with a frequency of $24.2 \mathrm{kHz}$. The rastering is done with the help of the two air core magnets, one for the horizontal direction and one for the vertical direction, and the raster pattern generator [47]. The details of the Hall C raster generator are given in [48]. In Hall C the "fast" rastering system is used for solid and cryogenic targets. In our experiment, the "fast" rastering was required only for a few, relatively thin, targets $\left(\mathrm{CH}_{2}\right.$ and $\left.{ }^{28} \mathrm{Si}\right)$.

The "slow" rastering is used for protection of the beam dump. The structural components of this system are the same as the "fast" one. However it only produces the $100 \mathrm{~Hz}$ rastering pattern. The "slow" rastering is usually required in experiments with the high currents $(>100 \mu \mathrm{A})$. Since the maximum current used in E01-011 was $30 \mu \mathrm{A}$, the usage of the "slow" rastering was not necessary.

\subsection{Experiment kinematics}

Figure 2.3.1 shows the schematic of the elementary reaction $\left(e, e^{\prime} K^{+}\right)$with the kinematical characteristics. A high-intensity, quasi-continuous, unpolarized $1.85 \mathrm{GeV}$ electron beam, with an energy spread less than or equal to $3 \cdot 10^{-5} \mathrm{GeV}$, is incident on the target. Upon interaction with the nuclear matter, taking into account the spectrometer acceptances, the electron recoils with a momentum ranging from 0.245 to $0.455 \mathrm{GeV} / \mathrm{c}$ centered on $0.35 \mathrm{GeV} / \mathrm{c}$. At the same time a virtual photon is released in the energy range from 1.4 to $1.6 \mathrm{GeV}$ with the central value of $1.5 \mathrm{GeV}$. This high-energy virtual photon 
interacts with the proton in the nucleus and, via associated mechanism (Fig. 1.5.2), results in a $1.2 \mathrm{GeV} / \mathrm{c}$ positive kaon and a $\Lambda$ at low or zero momentum.

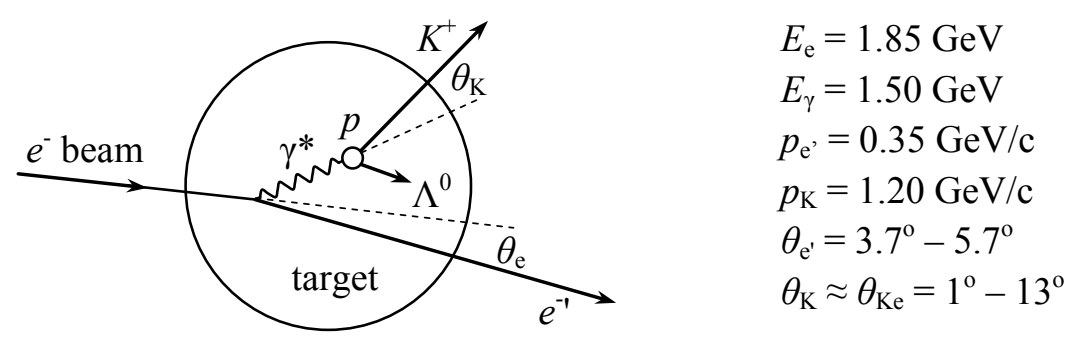

Figure 2.3.1 Schematics of E01-011 kinematics.

This kinematics was specifically chosen due to several factors intended to maximize the kaon yield and maximize $\Lambda$ hypernuclei production:

1. The elementary cross section of $\Lambda$ photoproduction $p\left(\gamma, K^{+}\right) \Lambda$ measured by the SAPHIR group [49] showed maximum cross-section values for photon energies $E_{\gamma}$ ranging from $1.1 \mathrm{GeV}$ to $1.6 \mathrm{GeV}$ (Fig. 2.3.2). The corresponding momentum of the kaon in such case ranges from $0.7 \mathrm{GeV} / \mathrm{c}$ to $1.6 \mathrm{GeV} / \mathrm{c}$. The hypernuclear cross section however becomes greater with increasing energy of the virtual photon, due to the decrease of the recoil momentum.

2. The momentum transfer to the $\Lambda$ hyperon, (Fig. 1.5.3), must be small to maximize the $\Lambda$ hypernuclei yield. A higher energy of the virtual gamma leads to lower momentum transfer. That means that for higher $E_{\gamma}$ the hypernuclear production yield increases.

3. To maximize the $\Lambda$ yield the kaon momentum also has to be carefully optimized. Since the E01-011 is a coincidence experiment, where the coincident in-time 
recoil electron and kaon must be measured, it is important to keep the kaon rate as high as possible. For a given flight path in the hadron spectrometer the kaon survival rate increases with higher kaon momentum.

4. To detect recoil electrons the experiment employed the Enge Split-Pole Spectrometer (ENGE), which was previously used in the HNSS experiment [12]. Originally the ENGE magnet was utilized at the Cyclotron Institute at Texas A\&M University, College Station, TX. The designed central momentum of the spectrometer was $276 \mathrm{MeV} / \mathrm{c}$. To fit in the momentum acceptance of the ENGE spectrometer, the kinematics was adjusted to yield $0.35 \mathrm{GeV}$ scattered electrons. With the energy of the incident electron beam at $1.85 \mathrm{GeV}$, the virtual gamma acquires an energy around $E_{\gamma}=1.5 \mathrm{GeV}$, which falls on the plateau of the cross section distribution (see Fig. 2.3.2). The corresponding kaon momentum is centered at approximately $1.2 \mathrm{GeV} / \mathrm{c}$. The beam energy had to be optimized to avoid the reaction channels that allow particles other than $\Lambda$ to be produced. Additionally, the bremsstrahlung cross section increases as the energy of the incident electron increases. The energy of the produced photons can reach values up to the energy of the beam. The existence of high-energy bremsstrahlung photons raises the likelihood of electron-positron pair production, which can obviously become a source of background. Apart of bremsstrahlung another dominant source of background in the electron arm is Møller scattering. The consequences caused by the individual contributions of each of these background sources are in detail discussed later in the text. 


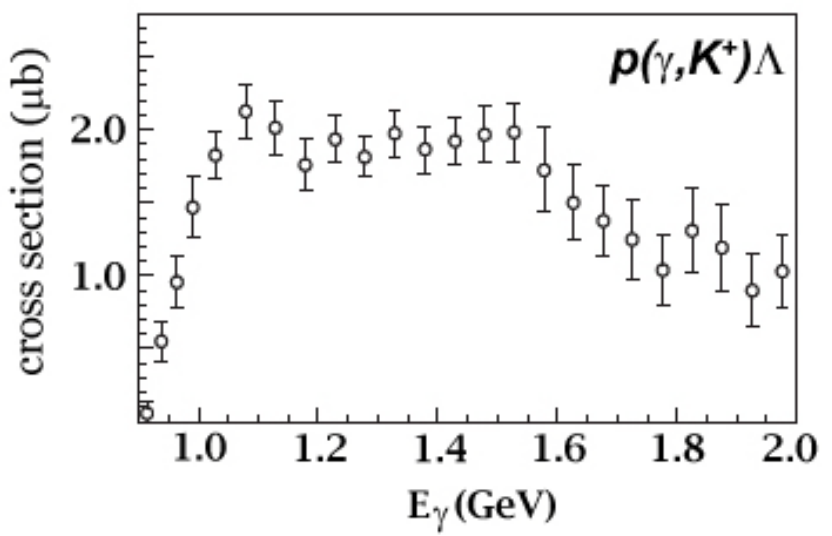

Figure 2.3.2 Total cross section of $\Lambda$ photoproduction reaction $p\left(\gamma, K^{+}\right) \Lambda$ [49].

According to distorted-wave impulse approximation (DWIA) calculations [50], the cross section of the ${ }_{\Lambda}^{12} \mathrm{~B}$ ground state doublet has the maximum at zero scattered kaon angle, $\theta_{K}$. The theoretical angular dependence on the $\theta_{K}$ of the cross section is shown in Fig. 2.3.3. The angle $\theta_{K}$ is counted with respect to the direction of the virtual photon.

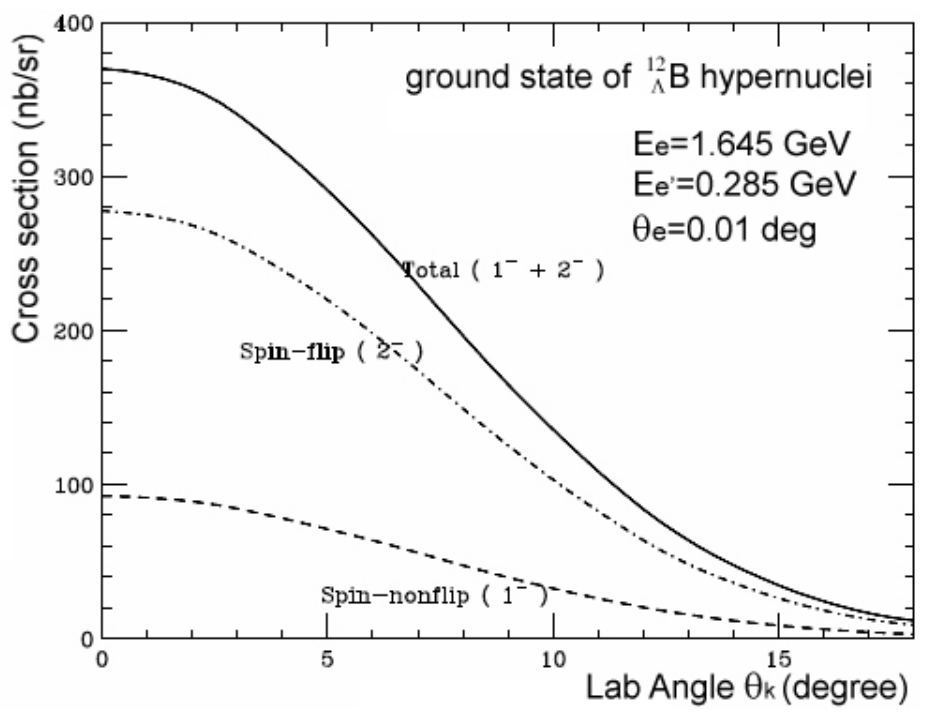

Figure 2.3.3 Kaon angular distribution of ${ }_{\Lambda}^{12} \mathrm{~B}$ ground state doublet cross section for ${ }^{12} \mathrm{C}\left(e, e^{\prime} K^{+}\right){ }_{\Lambda}^{12} \mathrm{~B}$ reaction [50]. 
Using $Q^{2}=4 E_{e} E_{e^{\prime}} \sin ^{2}\left(\theta_{e}\right)$, and employing Equations (2.2), (2.3), and (2.4) we can obtain an expression for the virtual photon flux as a function of electron scattering angle $\theta_{e}[51]:$

$$
\begin{aligned}
\Gamma\left(\theta_{e}\right)=\frac{\alpha}{4 \pi^{2} \omega} & {\left[\frac{E_{e}^{2}+E_{e^{\prime}}^{2}}{2 E_{e}^{2}}\left(\frac{m_{e}^{2} \omega^{2}}{4 E_{e}^{2} E_{e^{\prime}}^{2}}+\sin ^{2} \frac{\theta_{e}}{2}\right)^{-1}\right.} \\
& -\frac{E_{e^{\prime}}^{2}}{E_{e}^{2}} \frac{m_{e}^{2} \omega^{2}}{4 E_{e}^{2} E_{e^{\prime}}^{2}}\left(\frac{m_{e}^{2} \omega^{2}}{4 E_{e}^{2} E_{e^{\prime}}^{2}}+\sin ^{2} \frac{\theta_{e}}{2}\right)^{-2} \\
& \left.-\frac{\left(E_{e}+E_{e^{\prime}}\right)^{2}}{4 E_{e}^{2}}\left(\frac{\omega^{2}}{4 E_{e} E_{e^{\prime}}}+\sin ^{2} \frac{\theta_{e}}{2}\right)^{-1}\right] .
\end{aligned}
$$

As we mentioned earlier, one of the two processes contributing to background is pair production associated with bremsstrahlung in the target. The flux of pair production $\Phi_{\text {pair }}$ in terms of bremsstrahlung flux $\Phi_{\text {brem }}$, pair production cross section $\sigma_{\text {pair }}$ and target radiation length $t$ is [52]

$$
\Phi_{\text {pair }}=\Phi_{b r e m} \sigma_{p a i r} t X_{0}
$$

To calculate the contribution of the bremsstrahlung scattering the following expression can be used [53]:

$$
\begin{aligned}
\frac{d \sigma}{d \Omega_{k} d_{k}} & =\frac{2 \alpha^{3}}{\pi k} \frac{E^{2}}{m^{4}}\left[\left(\frac{2 x(1-x)}{(1+l)^{2}}-\frac{12 l x(1-x)}{(1+l)^{4}}\right) G_{2}(\infty)\right. \\
& \left.+\left(\frac{2 x^{2}-2 x+1}{(1+l)^{2}}-\frac{4 l x(1-x)}{(1+l)^{4}}\right)\left(X-2 Z^{2} f(\alpha Z)^{2}\right)\right]
\end{aligned}
$$


with parameters $y=k / E, l=\theta_{k}^{2} E / m^{2}$ and atomic form factors:

$$
\begin{gathered}
G_{2}(\infty)=G_{2}^{\text {elastic }}(\infty)+G_{2}^{\text {inelastic }}(\infty)=Z^{2}+Z, \\
X=\int_{t_{\min }^{\prime}}^{m^{2}(1+l)^{2}}\left[G_{2}^{\text {elastic }}(t)+G_{2}^{\text {inelastic }}(t)\right] \frac{t-t_{\min }^{\prime}}{l^{2}} d t .
\end{gathered}
$$

The angular dependence of the virtual photon flux (2.3.1) and bremsstrahlung scattering (2.3.3) is shown in the Fig. 2.3.4.

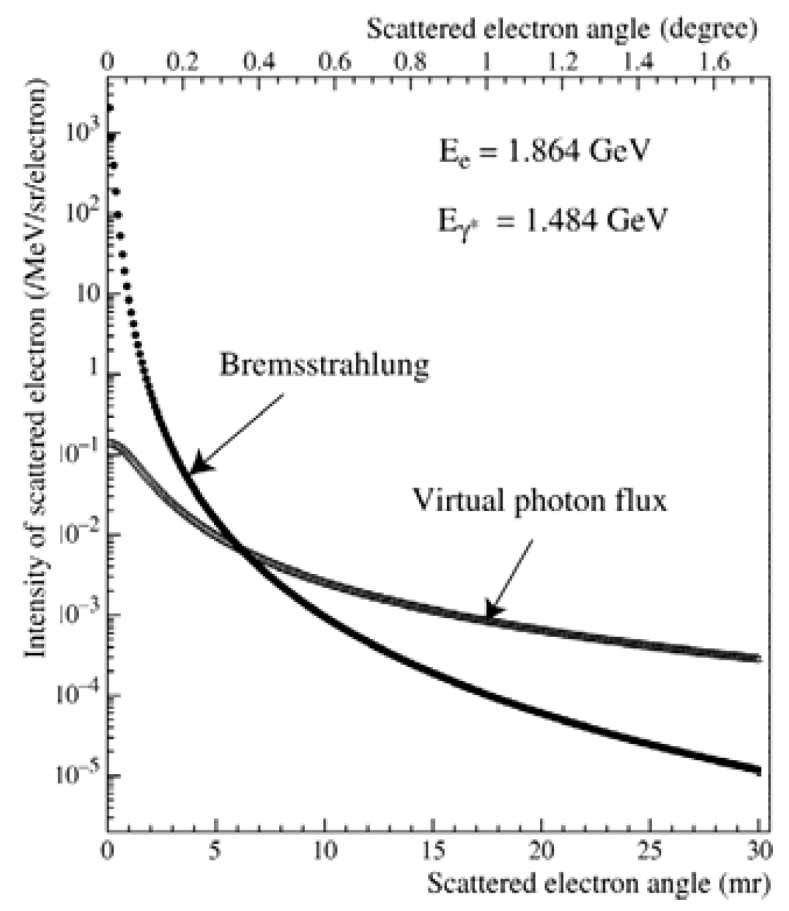

Figure 2.3.4 Angular distribution of the virtual photon flux and bremsstrahlung scattering [4].

As we can see from the figure, the flux of the virtual photons reaches its maximum at zero scattered electron angle. Since the cross section of hypernuclei electroproduction is directly proportional to the flux, we wish to detect both scattered electron and kaon at forward angle to maximize the cross section. However, in the forward angle 
configuration the bremsstrahlung intensity peaks at its maximum value. The previous hypernuclear experiment, HNSS, performed with recoil electron detection at zero degrees, reported electron rates in the order of several hundred MHz. The zero-degree electron tagging method used in that experiment was limited by the accidental rate from bremsstrahlung electrons. To reduce the accidental rate, the tilting of the electron spectrometer was introduced. The details of the "tilt" method will be described further.

The experiment used several targets for calibration and production purposes (Table 2.3.1). Most of the targets were enriched to have a higher purity. The $\mathrm{BeO}$ target was used for beam calibration, the $\mathrm{CH}_{2}-$ for kinematics and spectrometer optics calibrations, light targets $(6<Z<28)$ were utilized for spectroscopy study and heavier targets $(28<Z<208)$ were employed for rate studies. The thickness of the targets ranged from $46 \mathrm{mg} / \mathrm{cm}^{2}$ to $189 \mathrm{mg} / \mathrm{cm}^{2}$, while the current was between $0.2 \mu \mathrm{A}$ and $30 \mu \mathrm{A}$. For each of the targets, the current was tuned to provide a maximum kaon yield and at the same time to preserve the target material from overheating and melting. The numbers had to be carefully adjusted to keep the signal to noise ratio as high as possible. The maximum values of the beam current were limited by the data acquisition (DAQ) rate. For the high currents the DAQ rates were dominated by the accidental coincidence online trigger initiated due to the presence of other positive particles than kaons: positrons, pions, and protons. 
Table 2.3.1 Parameters of the targets used in the experiment.

\begin{tabular}{|c|c|c|c|c|c|c|}
\hline Target & $\begin{array}{c}\text { Purity } \\
(\%)\end{array}$ & $\begin{array}{l}\text { Thickness } \\
\left(\mathrm{mg} / \mathrm{cm}^{2}\right)\end{array}$ & $\begin{array}{c}\text { Current } \\
(\mu \mathrm{A})\end{array}$ & Raster & Data type & Purpose \\
\hline $\mathrm{BeO}$ & 99.5 & 143.6 & N/A & $\mathrm{N} / \mathrm{A}$ & test & beam calibration \\
\hline $\mathrm{CH}_{2}$ & $\mathrm{~N} / \mathrm{A}$ & 460 & 1.5 & $\mathrm{ON}$ & production & mass calibration \\
\hline${ }^{6} \mathrm{Li}$ & & 164 & 30 & $\mathrm{ON}$ & production & spectroscopy \\
\hline${ }^{7} \mathrm{Li}$ & & 189 & 27 & $\mathrm{ON}$ & production & spectroscopy \\
\hline${ }^{9} \mathrm{Be}$ & 99.0 & 189 & 19 & OFF & production & spectroscopy \\
\hline${ }^{10} \mathrm{~B}$ & 99.9 & 100 & 26 & ON & production & spectroscopy \\
\hline${ }^{12} \mathrm{C}$ & & 101.7 & 26 & OFF & production & spectroscopy \\
\hline${ }^{28} \mathrm{Si}$ & 99.9 & 50 & 18 & ON & production & spectroscopy \\
\hline${ }^{51} \mathrm{~V}$ & 99.7 & 59.6 & 18 & OFF & production & rate study \\
\hline${ }^{89} \mathrm{Y}$ & 99.9 & 56 & 13 & OFF & production & rate study \\
\hline${ }^{208} \mathrm{~Pb}$ & & 283 & 0.3 & OFF & production & rate study \\
\hline
\end{tabular}

\subsection{Experimental equipment}

The E01-011 hypernuclear experiment equipment consisted of a splitter system, a hadron spectrometer (HKS), and electron spectrometer (ENGE) (Fig. 2.4.1) [39].

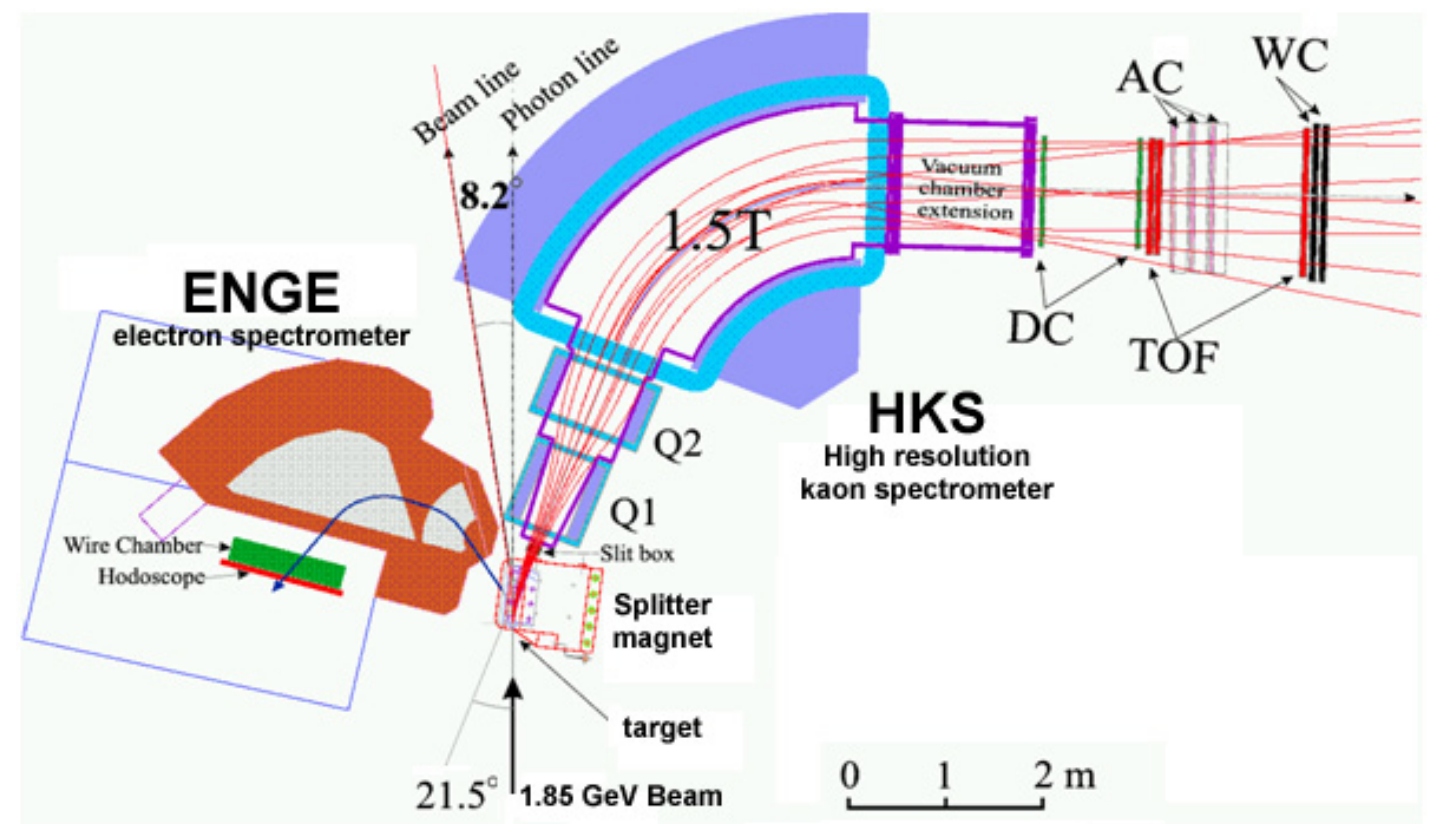

Figure 2.4.1 The schematics of the E01-011 experimental setup [39]. 
Each of these components consists of sets of steering magnets, supplementary instruments, and particle detectors. The recoil electrons and positively charged hadrons (pions, protons, and kaons) are the reaction products, caused by the $1.85 \mathrm{GeV}$ electron beam bombarding the target. The splitter system steers the negative particles towards the electron spectrometer system and deflects the positive particles into the hadron spectrometer system. Both spectrometers bend particle trajectories in the horizontal plane. The spectrometer system on the electron side has a slight vertical tilt to reduce accidental background. The components of spectrometers are built with the thought of searching for coincident, in-time, recoil electrons and kaons.

\section{Splitter system}

The splitter system utilized in the experiment consisted of a $\mathrm{C}$ type dipole magnet and a target chamber (Fig. 2.4.2). A CAD drawing and a photograph of the assembled magnet in front of HKS and ENGE spectrometers are shown in Fig. 2.4.2. The schematic drawing of the magnet with view from the top is presented in Fig. 2.4.3.

The purpose of the splitter magnet was to deflect the recoil electrons and positive kaons towards the corresponding spectrometers. After passing through the splitter, the electron beam was bent about 8.2 degrees to the left with respect to its initial direction, labeled as "photon line" in Fig. 2.4.1. With the help of a chicane consisting of additional dipole magnets, DZ and EZ, located behind the setup, the beam was deflected back to the photon line and directed to the beam dump. The use of the splitter magnet greatly benefited the big ENGE and HKS spectrometers positioned at large physical angles with 
respect to the beam trajectory. The splitter magnet was able to steer the forward scattered particles of opposite charges over a very limited space.
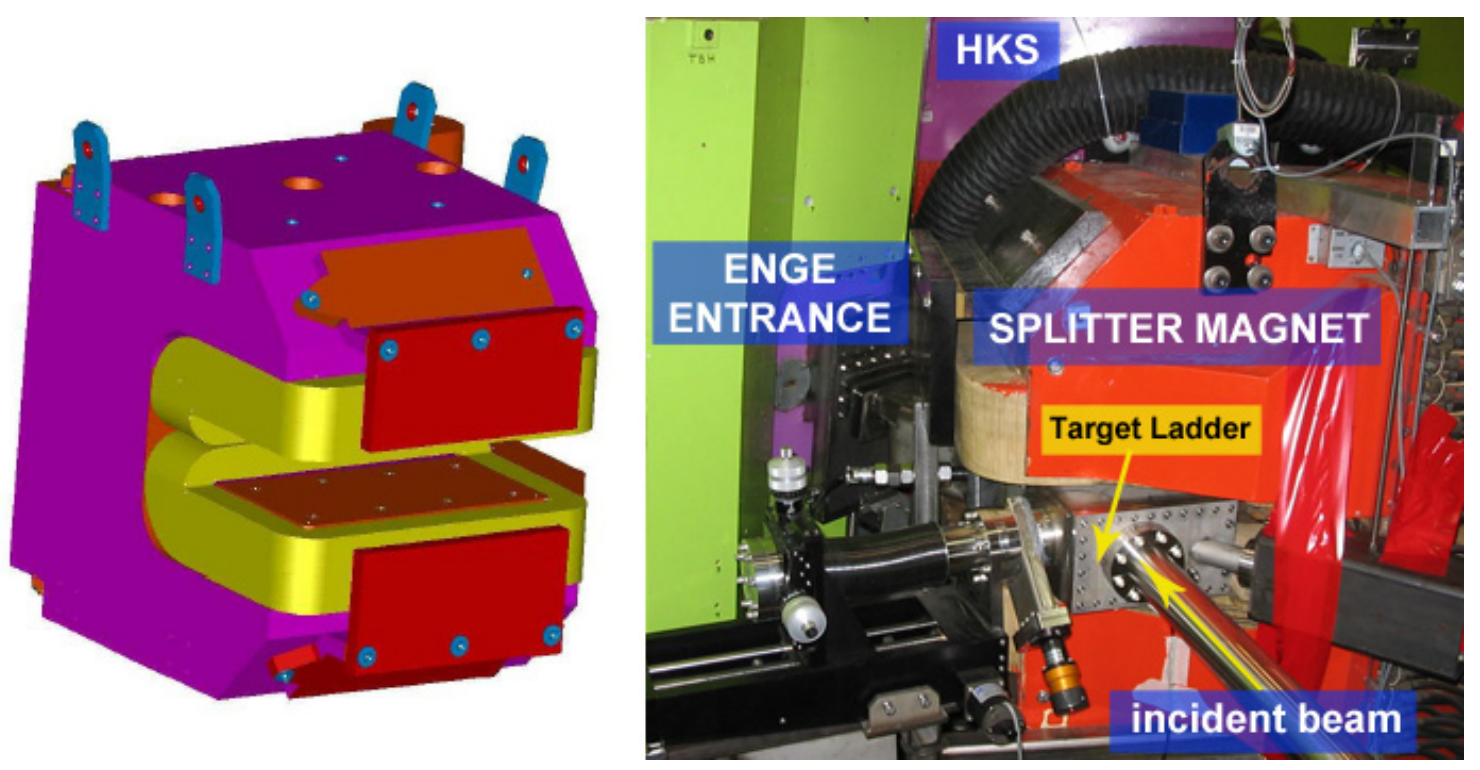

Figure 2.4.2 Splitter magnet. Schematic design on the left and the actual photo on the right [56].

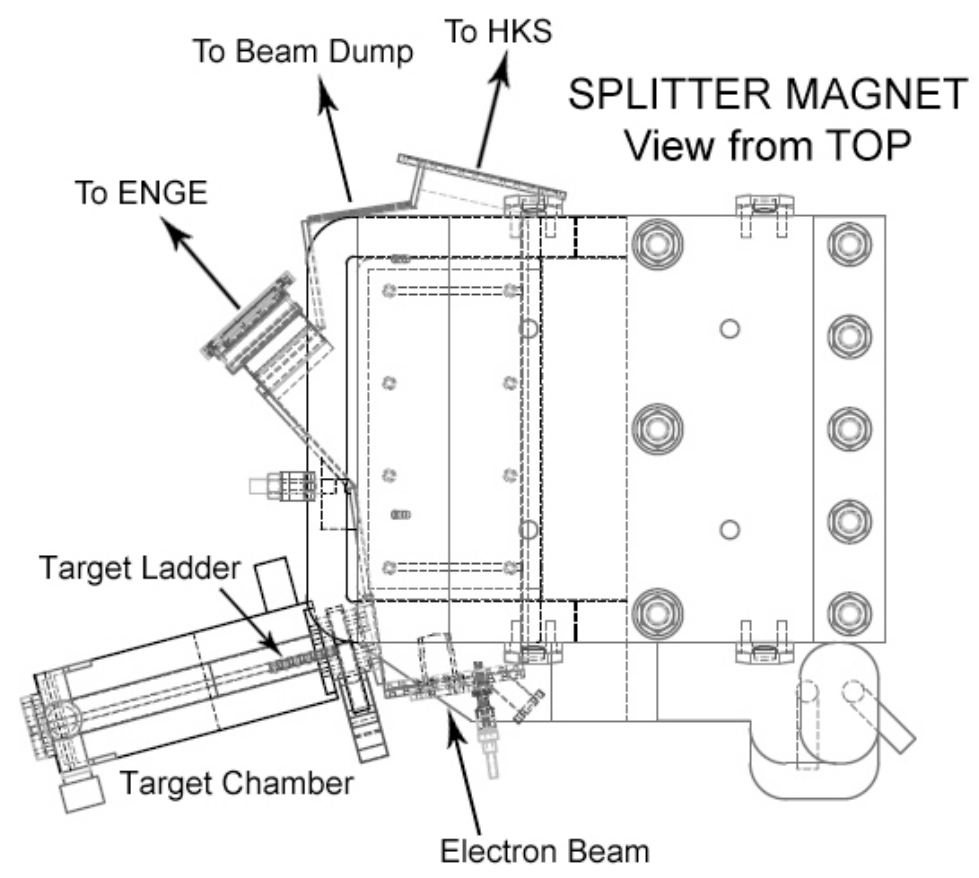

Figure 2.4.3 Schematic, "TOP view" of the splitter magnet and target chamber [56]. 
The magnet was previously used in the HNSS experiment. To accommodate the new geometry including the tilt of the ENGE spectrometer and the large acceptance of the HKS, the pole gap of the magnet was extended. It was almost doubled from $7.6 \mathrm{~cm}$ to $15.24 \mathrm{~cm}$. The magnetic field inside of the magnet was carefully mapped [54]. By initial design the magnet was supposed to operate at a central magnetic field of $1.546 \mathrm{~T}$. As a result of beam transportation problems through the chicane, the operational magnetic field had to be raised by $8.5 \%$ to a field of $1.672 \mathrm{~T}$. The new setting exceeded the maximum range of the mapped magnetic field values. Therefore the new value, $1.672 \mathrm{~T}$, was deduced from TOSCA calculations [54]. Basic parameters of the splitter magnet are shown in the Table 2.4.1.

Table 2.4.1 Parameters of the splitter magnet.

\begin{tabular}{cccccc}
\hline Pole gap & Maximum $B$ & Designed $B$ & Operational $B$ & $\begin{array}{c}\text { Operational } \\
\text { current }\end{array}$ & Weight \\
& & & & $942 \mathrm{~A}$ & $6.35 \mathrm{ton}$ \\
\hline
\end{tabular}

Throughout the entire experiment the magnetic field inside of the splitter magnet was monitored with a hall probe. The stability of the operational magnetic field in the magnet was very crucial. In case of a field drift higher than $\pm 10^{-4}$, or \pm 1.5 Gauss, the operational current had to be adjusted back to its nominal value, $942 \mathrm{~A}$.

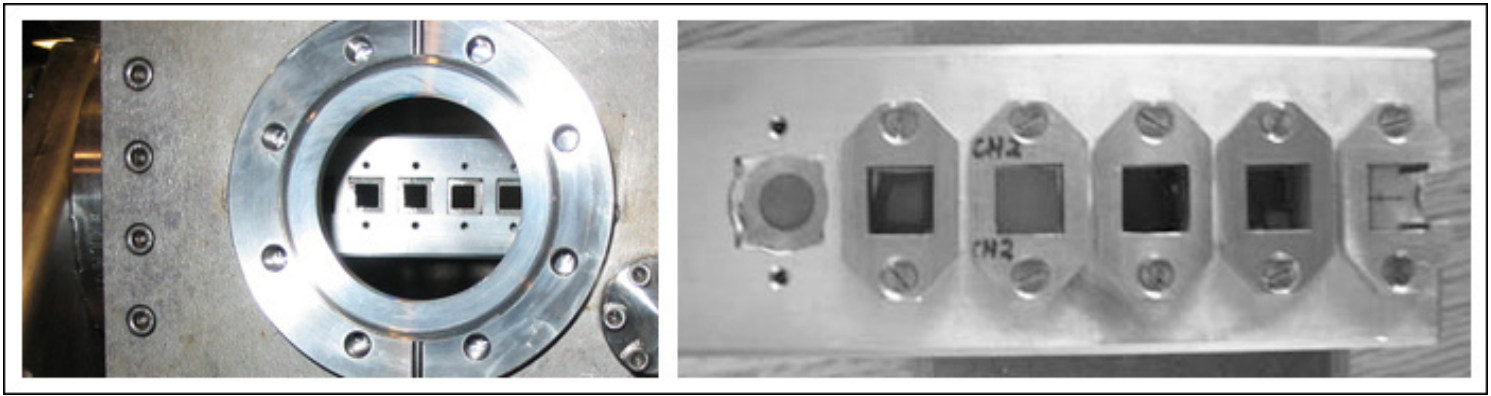

Figure 2.4.4 Target ladder inside of the chamber with empty target holes on the left and with mounted targets on the right [56]. 
The target chamber was installed in the gap, right at the field boundary near the beam entrance of the magnet (Fig. 2.4.4). By design, the electron beam had to hit the target perpendicularly. Because of the fringe field of the splitter, the beam was slightly steered to the side. To compensate the steering effect and align the electron beam back on its initial trajectory a small dipole magnet, DW, was used. It was installed right in front of the target. The left photo on the Fig. 2.4.4 shows the target ladder inside of the target chamber, mounted in the magnet's gap. The $220 \mathrm{~mm} \times 50 \mathrm{~mm}$ aluminum target ladder could hold six square targets. In total, four ladders were prepared for the experiment, with different targets mounted on each ladder [55]. All targets had an area of $1.5 \mathrm{~cm} \times 1.5 \mathrm{~cm}$. The target ladder could be remotely moved with a stepper motor. It also could be completely retracted into a small chamber behind a vacuum valve. This allowed exchange of ladders without breaking the spectrometer vacuum system. By moving the ladder in the horizontal direction the different targets could be exposed to the beam during the experiment. A visual inspection of the targets was possible with the help of a CCD camera. The target ladder assignment is shown in the Table 2.4.2.

Table 2.4.2 Target assignment on the target ladder [56].

\begin{tabular}{|c|c|c|c|c|c|c|}
\hline \multirow[b]{2}{*}{ Ladder } & \multicolumn{6}{|c|}{ Position on the ladder } \\
\hline & 1 & 2 & 3 & 4 & 5 & 6 \\
\hline L1 & $\mathrm{BeO}$ & Empty & ${ }^{12} \mathrm{C}$ & $\mathrm{CH}_{2}$ & ${ }^{9} \mathrm{Be}$ & ${ }^{28} \mathrm{Si}$ \\
\hline L2 & $\mathrm{BeO}$ & $\mathrm{CH}_{2}$ & $\mathrm{CH}_{2}$ & $\mathrm{CH}_{2}$ & $\mathrm{CH}_{2}$ & ${ }^{12} \mathrm{C}$ \\
\hline L3 & $\mathrm{BeO}$ & $\mathrm{CH}_{2}$ & ${ }^{11} \mathrm{~B}$ & ${ }^{10} \mathrm{~B}$ & ${ }^{9} \mathrm{Be}$ & ${ }^{28} \mathrm{Si}$ \\
\hline L4 & $\mathrm{BeO}$ & ${ }^{6} \mathrm{Li}$ & ${ }^{7} \mathrm{Li}$ & ${ }^{51} \mathrm{~V}$ & ${ }^{89} \mathrm{Y}$ & ${ }^{208} \mathrm{~Pb}$ \\
\hline
\end{tabular}




\subsubsection{HKS spectrometer}

The hadron spectrometer system consists of the high resolution kaon spectrometer (HKS) and the associated set of focal plane detectors. Two quadruple magnets, Q1 and Q2, together with the dipole magnet, D, comprise the HKS spectrometer (Fig. 2.4.1). The parameters of the HKS spectrometer and the specifications of the magnets are shown in Tables 2.4.3 and 2.4.4, respectively.

Table 2.4.3 Parameters of the HKS spectrometer.

\begin{tabular}{lc}
\hline Item & HKS \\
\hline Configuration & QQD with $70^{\circ}$ horizontal bend \\
Central momentum & $1.2 \mathrm{GeV} / \mathrm{c}$ \\
Momentum dispersion & $4.7 \mathrm{~cm} \mathrm{per} 1 \%$ \\
Momentum acceptance & $\pm 12.5 \%(1.05 \mathrm{GeV} / \mathrm{c}-1.35 \mathrm{GeV} / \mathrm{c})$ \\
Momentum resolution $(\Delta p / p)$ & $2 \cdot 10^{-4}(\mathrm{FWHM})$ \\
Solid angle & $20 \mathrm{msr}$ with a splitter $/ 30 \mathrm{msr}$ without a splitter \\
Kaon detection angle & Horizontal at $7^{\circ}\left(1^{\circ}-13^{\circ}\right)$ \\
Flight path length & $10 \mathrm{~m}$ \\
Maximum magnetic field & $1.6 \mathrm{~T}$ \\
\hline
\end{tabular}

The HKS spectrometer was designed to operate at a central momentum of $1.2 \mathrm{GeV} / \mathrm{c}$ and to achieve a momentum resolution of $2 \cdot 10^{-4}$ (FWHM). It was aligned and tuned to detect positively charged particles, kaons, pions, and protons emitted at an angle from $1^{\circ}$ to $13^{\circ}$. That allowed avoiding positrons from bremsstrahlung produced at $0^{\circ}$.

The magnets were designed and produced by Mitsubishi Electric Corporation in Japan. They were tested and mapped before being shipped to Jefferson Lab. The dipole magnet was mapped again at Jefferson Lab for consistency of the test measurements by Mitsubishi. A photograph of the assembled HKS spectrometer inside of Hall C is shown 
in Fig. 2.4.5. A schematic representation of the QQD system of the spectrometer is depicted in Fig. 2.4.6 [39].

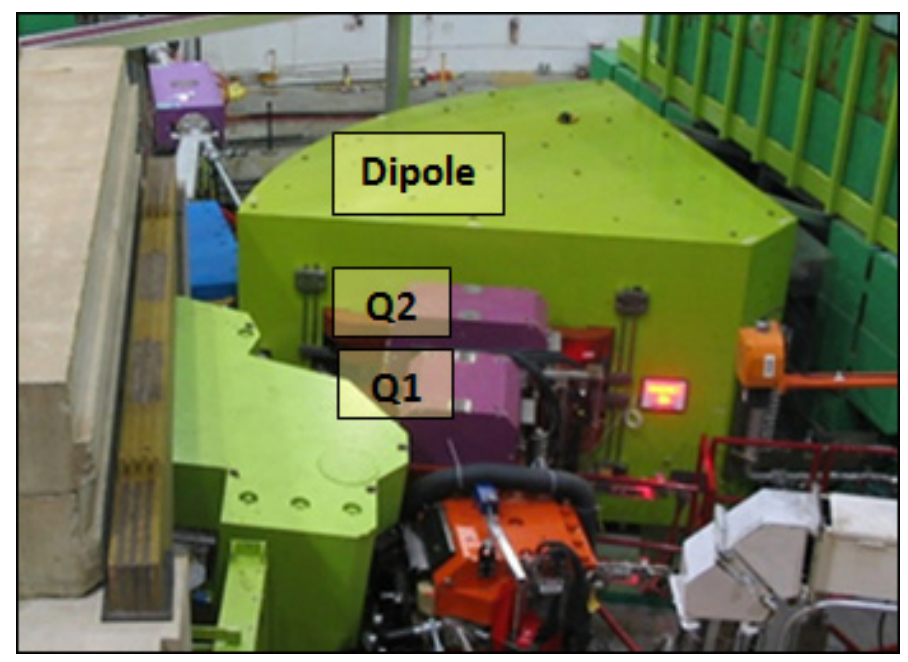

Figure 2.4.5 Photo of the HKS spectrometer inside of the Hall C [56]. The quadruple Q1 is placed behind the splitter magnet, and it is followed by quadruple Q2 and further by the dipole.

Table 2.4.4 Specifications of the HKS magnets: Q1, Q2, and D.

\begin{tabular}{|c|c|c|c|c|}
\hline Item & Q1 & Q2 & \multicolumn{2}{|c|}{$\mathrm{D}$} \\
\hline Bore radius $(\mathrm{mm})$ & 120 & 145 & \multicolumn{2}{|c|}{-} \\
\hline Pole gap height (mm) & - & - & \multicolumn{2}{|c|}{200} \\
\hline Pole length $(\mathrm{mm})$ & - & - & \multicolumn{2}{|c|}{1560} \\
\hline Max. Ampere turns (A·turns) & 224000 & 144000 & \multicolumn{2}{|c|}{291840} \\
\hline Number of turns & 256 & 320 & \multicolumn{2}{|c|}{256} \\
\hline $\begin{array}{l}\text { Conductor size and cooling } \\
\text { channel hole }(\mathrm{mm})\end{array}$ & $8 \times 8(\phi 6)$ & $13.5 \times 11.5(\phi 6.3)$ & \multicolumn{2}{|c|}{$22 \times 22(\phi 12)$} \\
\hline Field gradient $(\mathrm{T} / \mathrm{m})$ & 6.6 & 4.2 & \multicolumn{2}{|c|}{-} \\
\hline Max. field (T) & - & - & \multicolumn{2}{|c|}{1.53} \\
\hline Max. current (A) & 875 & 450 & \multicolumn{2}{|c|}{1140} \\
\hline \multirow[t]{2}{*}{ Resistance $(\mathrm{m} \Omega)$} & $181\left(\right.$ at $\left.55^{\circ} \mathrm{C}\right)$ & $119\left(\right.$ at $\left.45^{\circ} \mathrm{C}\right)$ & \multicolumn{2}{|c|}{$145\left(\right.$ at $\left.47.5^{\circ} \mathrm{C}\right)$} \\
\hline & & & Gap side & Yoke side \\
\hline Cooling water flow rate $(1 / \mathrm{mm})$ & 49.6 & 17.3 & 66.3 & 68.8 \\
\hline Pressure drop (MPa) & 0.36 & 0.38 & 0.32 & 0.35 \\
\hline Number of coolant circuits & 16 & 8 & 8 & 8 \\
\hline Total magnet weight (metric ton) & 8.2 & 10.5 & \multicolumn{2}{|c|}{210} \\
\hline
\end{tabular}


The magnets were controlled remotely from the Hall $\mathrm{C}$ counting house. To monitor the magnetic field during the experiment, hall probes were installed in each magnet. The operational values of currents and magnetic fields for all three magnets are shown in Table 2.4.5.

Table 2.4.5 Operational conditions of Q1, Q2, and D magnets during the run period.

\begin{tabular}{lccc}
\hline Item & Q1 & Q2 & D \\
\hline Current (A) & 597 & 401 & 1058 \\
Magnetic field (T) & -0.8648 & -0.1660 & 1.44031 \\
\hline
\end{tabular}
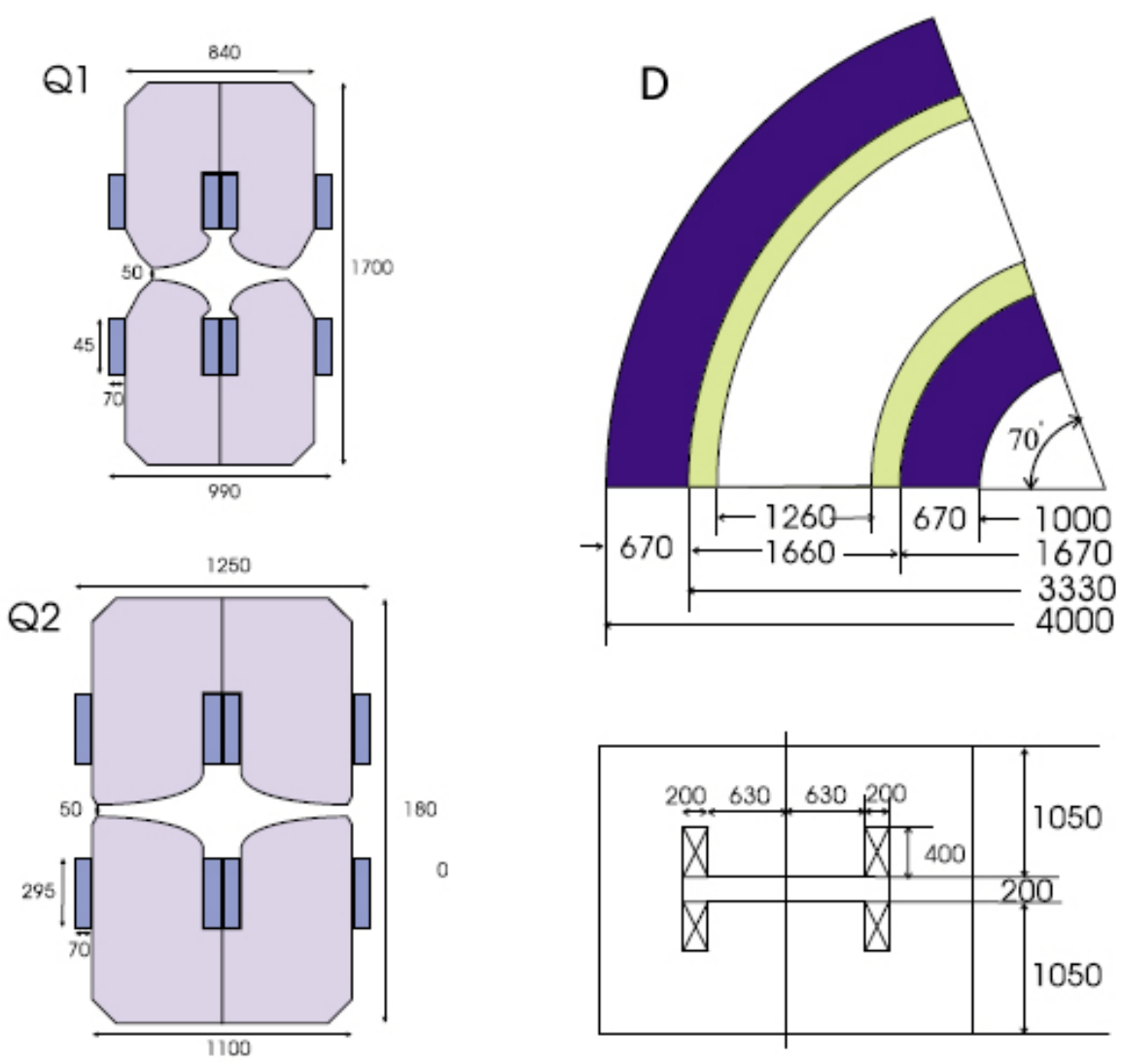

Figure 2.4.6 Schematic representation of the components of QQD HKS spectrometer [39]. (The units are given in $\mathrm{mm}$ ). 


\subsubsection{ENGE spectrometer}

The HKS experiment employed the Split Pole ENGE spectrometer previously used in HNSS. A sketch of the magnet is shown in Fig. 2.4 .7 [57,58]. It was given its name (Split Pole) because it contains two separate poles embraced by a single coil. The median plane in the split area is used for second-order electron focusing over the broad energy range that is accomplished by thoughtful choices of curvatures and locations of the pole boundaries. The ENGE is a horizontal bending spectrometer with a well defined focal plane. The size of the magnet is relatively small and the key advantage of the spectrometer is a high momentum resolution, $\Delta p / p=4 \cdot 10^{-4}$. At the central momentum of the magnet, the relative momentum uncertainty was around $130 \mathrm{keV} / \mathrm{c}$. The main characteristics of the ENGE magnet are presented in Table 2.4.6. Before the experiment, the magnet was powered and a $B-I$ excitation curve was measured (Fig. 2.4.8). The settings of the current in the magnet were adjusted to compensate for changes made to the splitter magnet. The operational magnetic field of the spectrometer was $1.5685 \mathrm{~T}$, with the current in the coils equal to $366.5 \mathrm{~A}$. 


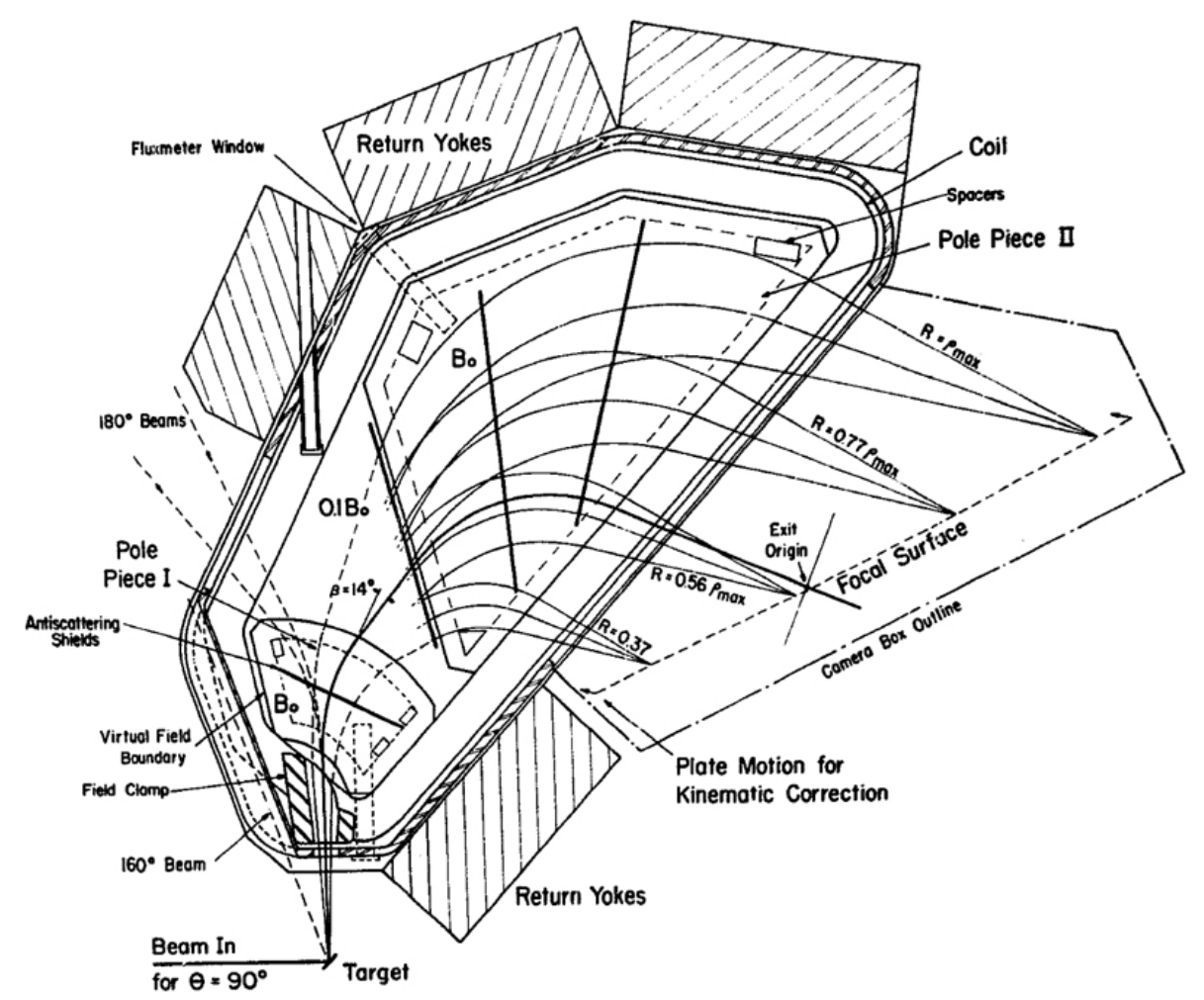

Figure 2.4.7 Sketch of Enge split-pole spectrometer (top view) [57,58].

Table 2.4.6 Enge split-pole spectrometer parameters.

\begin{tabular}{ll}
\hline Parameter & Value \\
\hline Pole gap & $46.6 \mathrm{~mm}$ \\
Momentum range & $228-338 \mathrm{MeV} / \mathrm{c}$ \\
Central designed and operational momenta & $276 \mathrm{MeV} / \mathrm{c}$ and $316 \mathrm{MeV} / \mathrm{c}$ \\
Momentum acceptance & $\pm 30 \%(221-441) \mathrm{MeV} / \mathrm{c}$ \\
Momentum resolution $(\Delta p / p)$ & $4 \cdot 10^{-4}(\mathrm{FWHM})$ \\
Solid angle acceptance & $1.6 \mathrm{msr}$ \\
Horizontal acceptance & $25 \mathrm{msr}$ \\
Vertical acceptance & $20 \mathrm{msr}$ \\
Total flight length & $\sim 5 \mathrm{~m}$ \\
Bend angle & $48.8^{\circ}$ \\
Dispersion & $1.5 \mathrm{~cm} / \%$ \\
Maximum field & $1.8 \mathrm{~T}$ \\
Maximum current & $500 \mathrm{~A}$ \\
Weight & $54.43 \mathrm{ton}$ \\
\hline
\end{tabular}




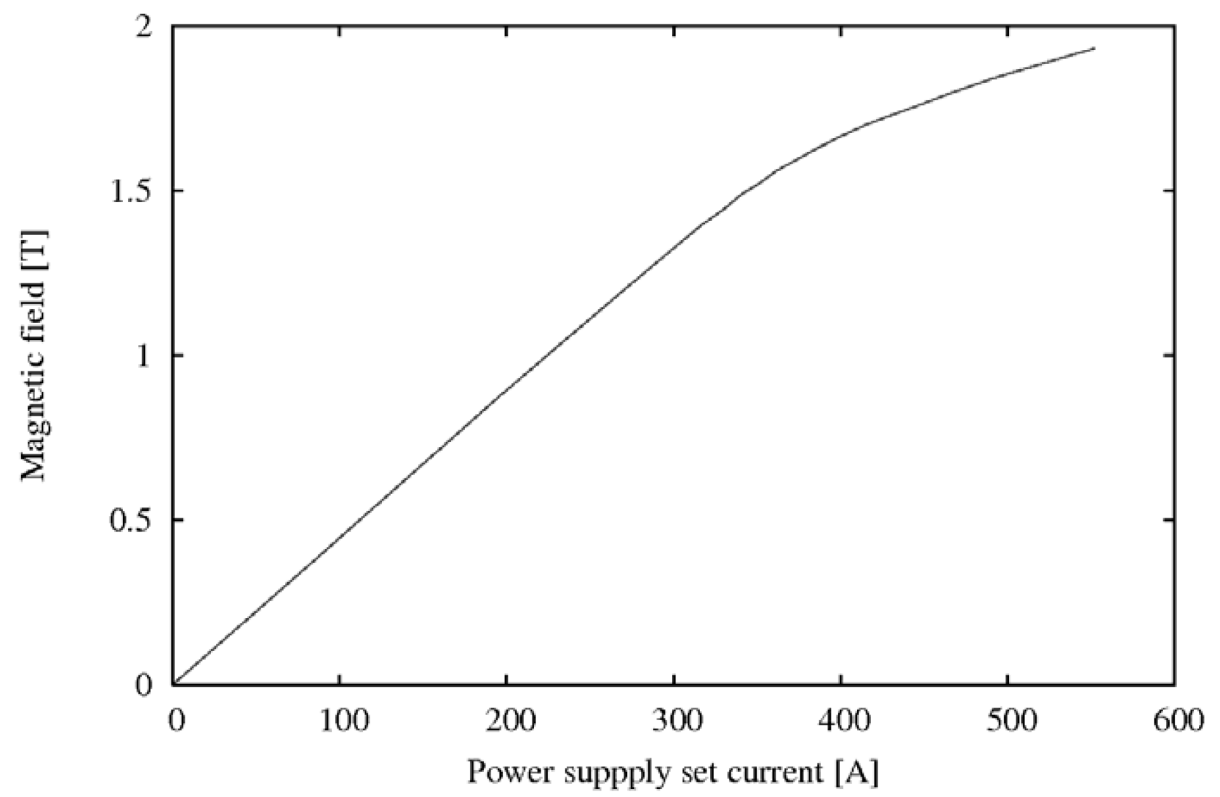

Figure 2.4.8 Magnetic field versus current (B-I) curve obtained at the JLab test facility [56].

\subsubsection{ENGE tilt method}

The new tilt method was introduced in order to avoid the accidental background rate associated with bremsstrahlung and Møller scattered electrons produced with momentum $200-400 \mathrm{GeV} / \mathrm{c}$ (see Section 2.3). The electron spectrometer, ENGE, was tilted vertically at $7.75^{\circ}$ with respect to the scattering plane (Fig. 2.4.9). A special tilting mechanism was developed (Fig. 2.4.10).

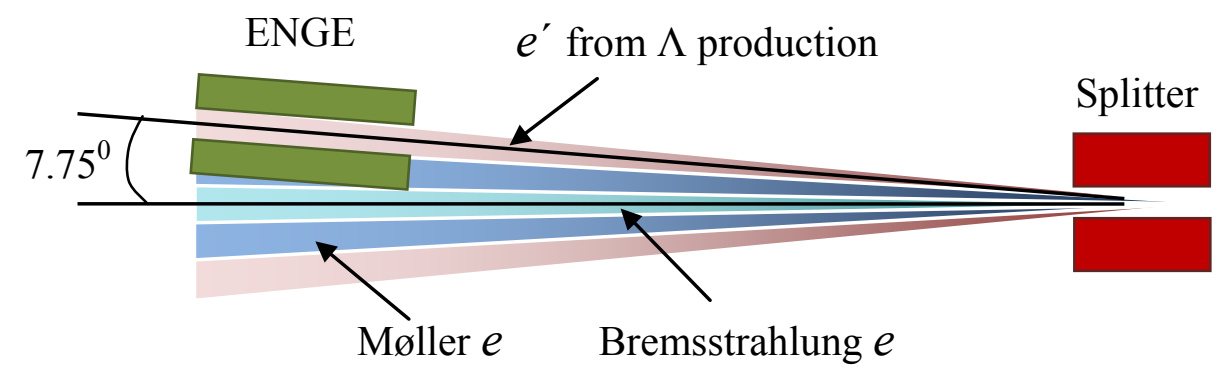

Figure 2.4.9 Schematic side view of the ENGE tilt method. 
The expected rates of the three processes: bremsstrahlung (Brems), Møller, and recoil electrons associated with virtual photon (VP) production, are shown in Fig. 2.4.11. The two dimensional angular profile is also presented in Fig. 2.4.11. The bremsstrahlung peaks at a forward angle, as does the virtual photon production. The Møller scattering electrons have their maximum at around 5 degrees. The rates were estimated for a 100 $\mathrm{mg} / \mathrm{cm}^{2}$ thick ${ }^{12} \mathrm{C}$ target with a $30 \mu \mathrm{A}$ current.

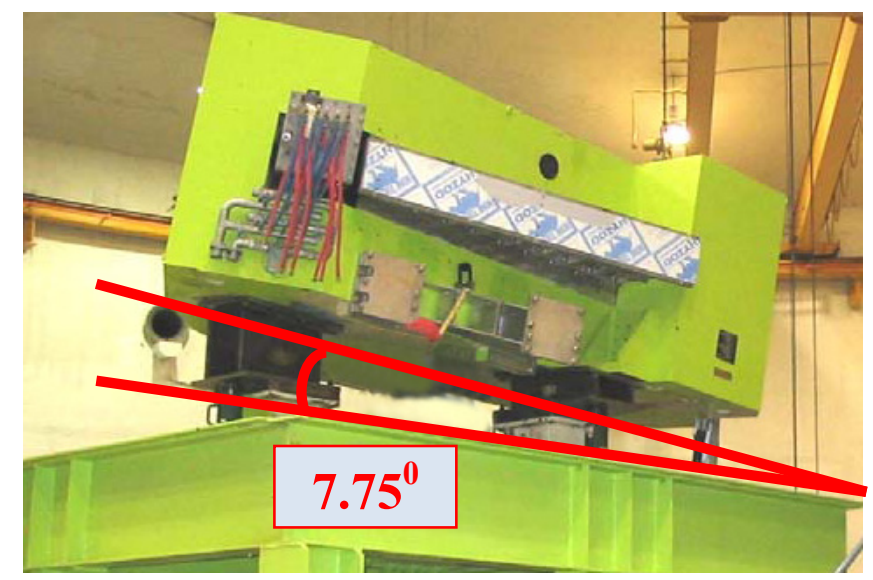

Figure 2.4.10 Tilted ENGE spectrometer [56]. Tile angle $=7.75^{\circ}$.
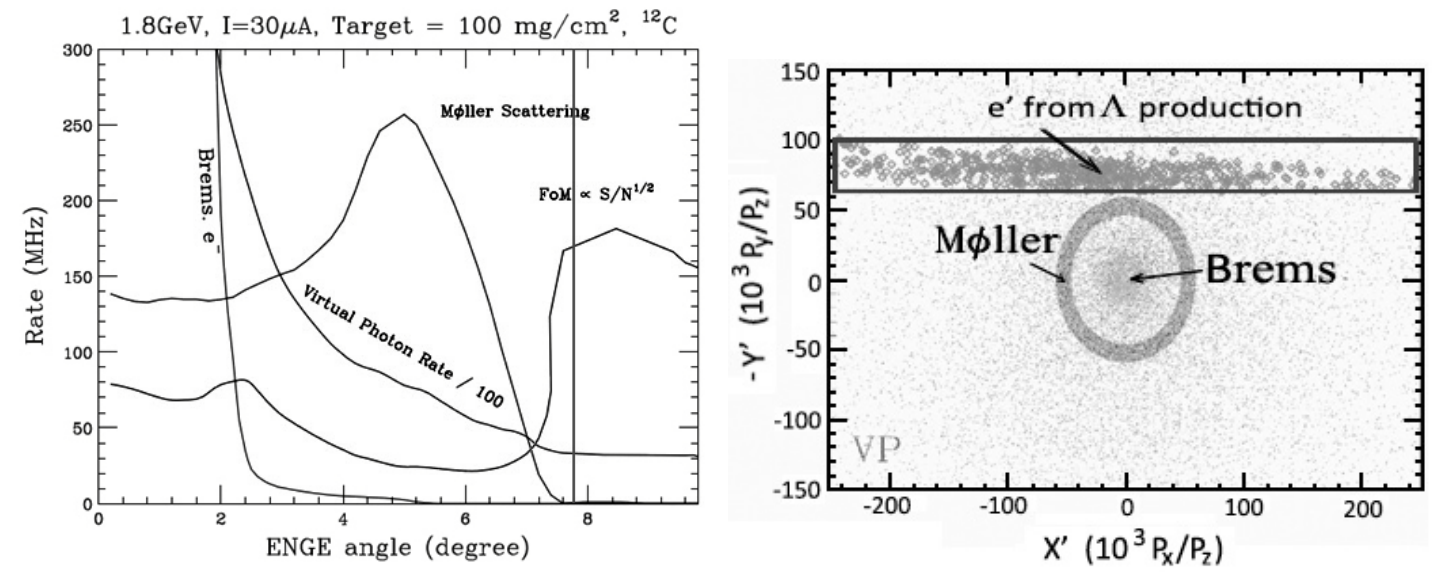

Figure 2.4.11 Left: Electron rate dependence on the ENGE tilt angle [56]. Right: Angular distribution of the scattered and accidental electrons [56]. The distributions are weighted by specific coefficients to make the plot readable. 
The Figure of Merit (FoM) is defined as $\mathrm{FoM}=S / \sqrt{N}$, where the signal $S$ is the rate of VP and noise $N$ is the same of the bremsstrahlung and Møller. As we can see from Fig. 2.4.11, the FoM reaches its maximum at around 8 degrees. Therefore the decision was made to tilt the ENGE spectrometer to accept the electrons at angle $7.75^{\circ}$. This corresponds to the selected rectangle on the right plot in Fig. 2.4.11. The RAYTRACE calculations performed by the collaboration determined the optimal value of this tilt. While the HNSS experiment reported an electron rate of the order of $200 \mathrm{MHz}$ with 0.6 $\mu \mathrm{A}$ beam on $22 \mathrm{mg} / \mathrm{cm}^{2}$ carbon target, the HKS measured roughly $2 \mathrm{MHz}$ rate on 100 $\mathrm{mg} / \mathrm{cm}^{2}$ thick target with $30 \mu \mathrm{A}$. Such a significant reduction, mostly as result of lowering the rate of accidental electrons, allowed increasing the current of the beam from $1 \mu \mathrm{A}$ (HNSS) to $30 \mu \mathrm{A}(\mathrm{HKS})$ and also made it possible to utilize thicker targets.

\subsubsection{Particle detectors}

\subsubsection{ENGE detector package}

The ENGE detector package consists of a drift chamber (EDC) for electron tracking, two sets of plastic scintillators, EHODO1 and EHODO2, that provide a timing measurement (Fig. 2.4.12), and a third scintillator for calibration. EDC is mounted directly on the ENGE exit gap. The chamber's plane was aligned with the exit plane of the spectrometer. The scintillator layers were mounted behind the EDC. 


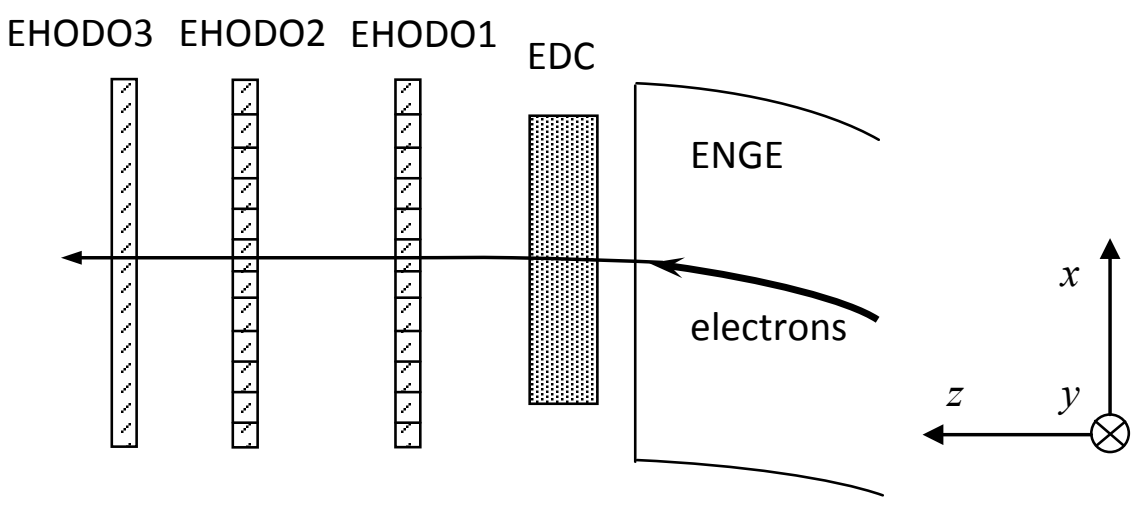

Figure 2.4.12 Enge detector package.

\section{ENGE drift chamber}

A drift chamber is a particle detector that employs the properties of the ionization process created by a particle traversing its gas. It is usually composed of many parallel wires arranged in a grid. Fig. 2.4.13 schematically shows the so-called honeycomb pattern of the EDC detector. A large electric potential difference is maintained between the anode and cathode wires. The electrons from the ionization are attracted to the sense wire. The large electric-field gradient near the sense wire results in an "avalanche" of ionization causing a large multiplication of the charge on the sense wire. The connected electronics measure the arrival time of the created signal. The difference between this time and the time when the particle traversed the cell, which is measured by other detectors, is used to reconstruct the nearest distance from the wire of the ionization trail. By employing several parallel layers of sense wires one can uniquely identify the trajectory of the passing particle. One of the common problems of drift chambers is the left-right ambiguity, which implies the inability to determine the location of the ionization origin 
due to the radial symmetry. To remove this ambiguity, a parallel layer of sense wires, shifted half of the cell size, is usually used. We refer to these layers as "primed" layers, e.g. $x^{\prime}$.

During development of the ENGE drift chamber the following requirements had to be taken into consideration: a) the momentum resolution $\Delta p / p$ had to be comparable to $4 \cdot 10^{-4}$ (FWHM), which required the position and angular resolution to be $100 \mu \mathrm{m}$ and 2 $\mathrm{mr}$ (rms) respectively; b) the chamber had to function for the large-angle (more than 50 degrees) incident trajectories; c) the detector had to be able to withstand the high single rates, up to $10 \mathrm{MHz}$. The chosen honeycomb cell structure (Fig. 2.4.13) is especially suitable for large incident angle trajectories.

The cell has a hexagonal configuration with side width $0.5 \mathrm{~cm}$. The chamber consists of 10 planes - $x x^{\prime} u u^{\prime} x x^{\prime} v v^{\prime} x x^{\prime}, 20 \mu \mathrm{m}$ diameter sense wires, $80 \mu \mathrm{m}$ diameter field wires and 4 layers of $80 \mu \mathrm{m}$ diameter shield wires. All wires are made of gold-plated tungsten. Wires in the $u$ and $v$ layers are tilted at $+30^{0}$ and $-30^{\circ}$ with respect to the $x$ layer.

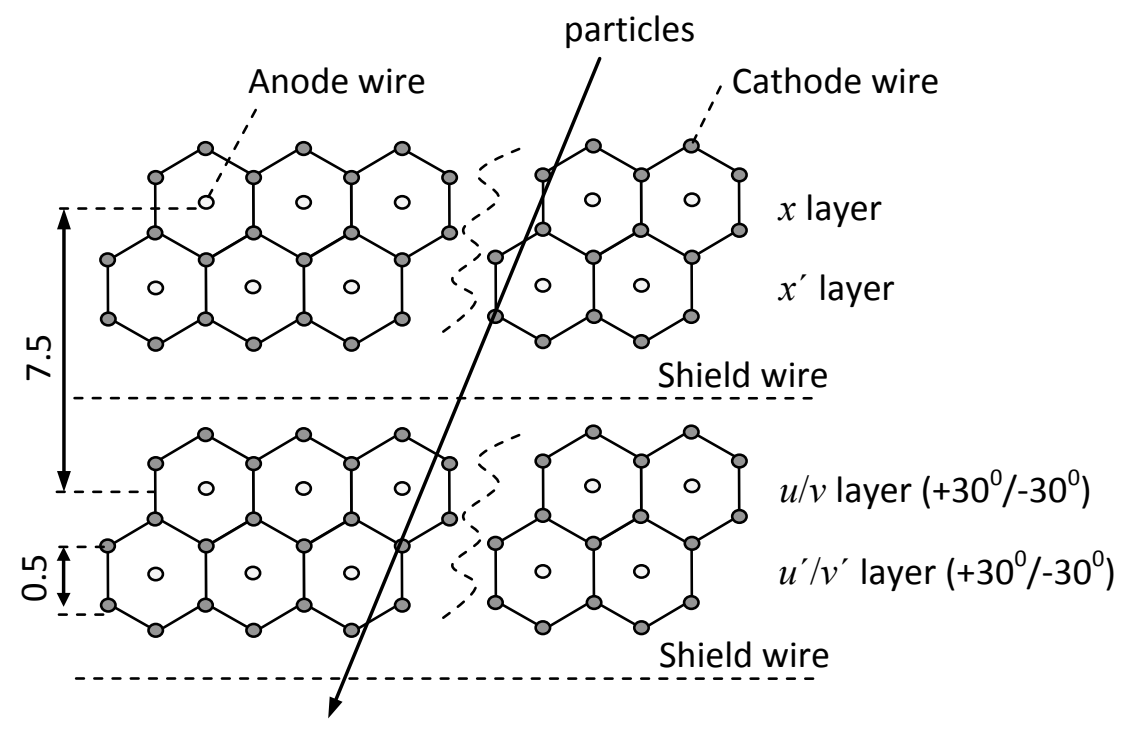

Figure 2.4.13 ENGE honeycomb drift chamber. (The units used are in $\mathrm{cm}$ ). 
The EDC is filled with an Argon/Ethan gas mixture in proportion of 50\% / 50\%. To prevent the aging of the detectors a small amount of alcohol vapor, about $1 \%$, was mixed to the gas. The effective volume of the chamber is $12^{\mathrm{H}} \times 100^{\mathrm{W}} \times 30^{\mathrm{T}} \mathrm{cm}^{3}$. The read-out electronics were comprised of 70 amplifier-discriminator cards (N277-L), with 35 cards at the bottom and another 35 at the top of the chamber. The N277L card used both $+5 \mathrm{~V}$ and $-5 \mathrm{~V}$ power input. The output signals from the cards were directed to $\mathrm{F} 1$ time-to-digital-converters (TDC) via 33 feet flat cables. The geometrical parameters of the EDC are summarized in the Table 2.4.7.

Table 2.4.7 ENGE honeycomb drift chamber (EDC) parameters.

\begin{tabular}{ll}
\hline Number of layers & 10 \\
Layer configuration & $x x^{\prime} u u^{\prime}\left(+30^{0}\right) x x^{\prime} v v^{\prime}\left(-30^{0}\right) x x^{\prime}$ \\
Effective volume & $12^{\mathrm{H}} \mathrm{cm} \times 100^{\mathrm{W}} \mathrm{cm} \times 30^{\mathrm{T}} \mathrm{cm}$ \\
Cell size & Side width $=0.5 \mathrm{~cm}$; hexagon (honeycomb) geometry \\
Anode wire & $20 \mu \mathrm{m}$ in diameter tungsten \\
Shield / field wire & $80 \mu \mathrm{m}$ in diameter tungsten \\
Gas & Argon-Ethane (50/50) \\
Gas pressure & $\sim 16 \mathrm{psi}$ \\
Operational HV & $-2200 \mathrm{~V}$ \\
Threshold & $2 \mathrm{~V}$ \\
Readout & $70 \mathrm{Nanometric} \mathrm{N277L} \mathrm{amplifier-discriminator} \mathrm{cards.} \mathrm{(35} \mathrm{top)/35} \mathrm{(bottom)}$ \\
\hline
\end{tabular}

A photograph of the drift chamber during the assembly and tests at Jefferson Lab is shown in the Fig. 2.4.14. 


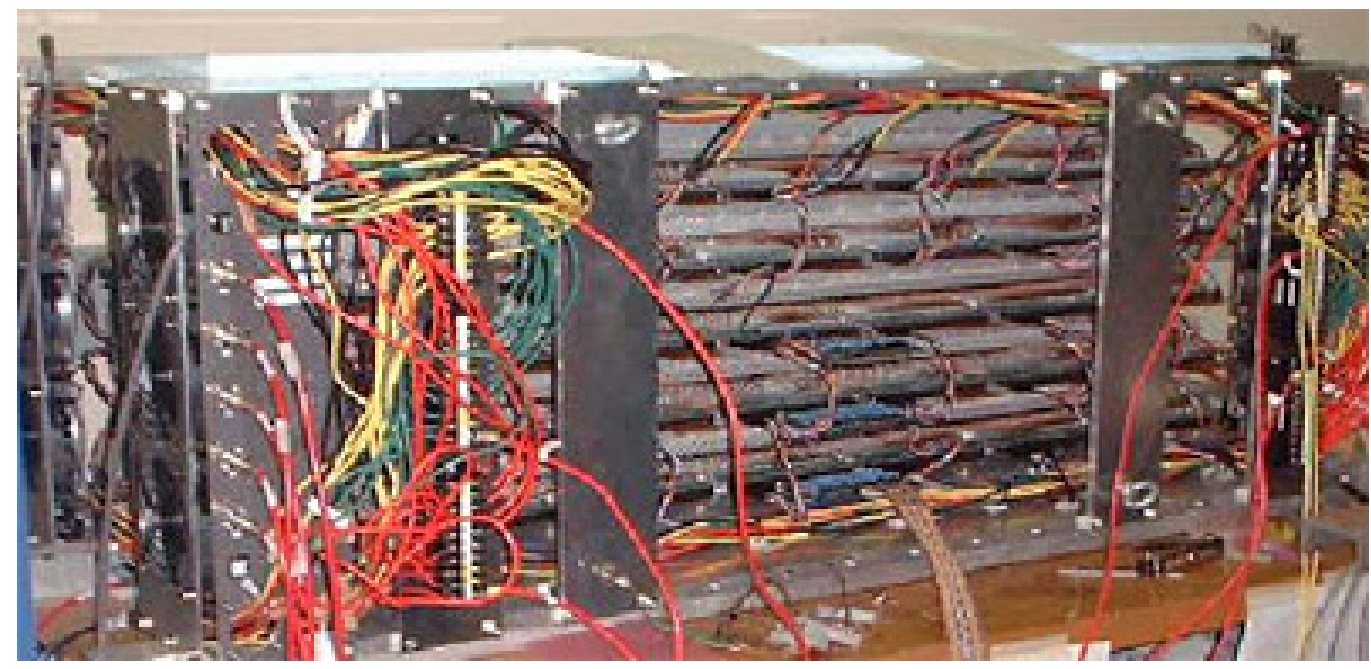

Figure 2.4.14 ENGE honeycomb drift chamber in EEL building at Jefferson Lab [56].

The average plane resolution of the chamber was reported from tests to be $390 \mu \mathrm{m}$ and the momentum resolution reached the designed value $4 \cdot 10^{-4}$ (FWHM).

\section{ENGE hodoscopes}

A hodoscope is a scientific instrument used for detection of charged particles, in particular, ionizing particles. It consists of scintillator slabs, which are usually a plastic, and photomultiplier tubes (PMT). As ionizing radiation traverses the plastic, its atoms are core-excited, and in the subsequent process of de-excitation visible light is emitted. The scintillator is made of a special kind of plastic called poly-vinyl toluene, PVT. The PVT is designed so that the light emitted inside the plastic experiences an internal reflection. Bouncing up and down along the slab it reaches the end of the plastic paddle where, with the help of a light guide, it couples with the PMT (Fig. 2.4.15). Usually the light is produced and collected very quickly, within a few nanoseconds. The photomultiplier tubes (PMT) operate under a high voltage and have a gain of about $10^{7}$. They are very 
sensitive to light and tuned to measure low signal light. The whole scintillating slab is thoroughly covered in a light-tight black coating to prevent background from external light sources.

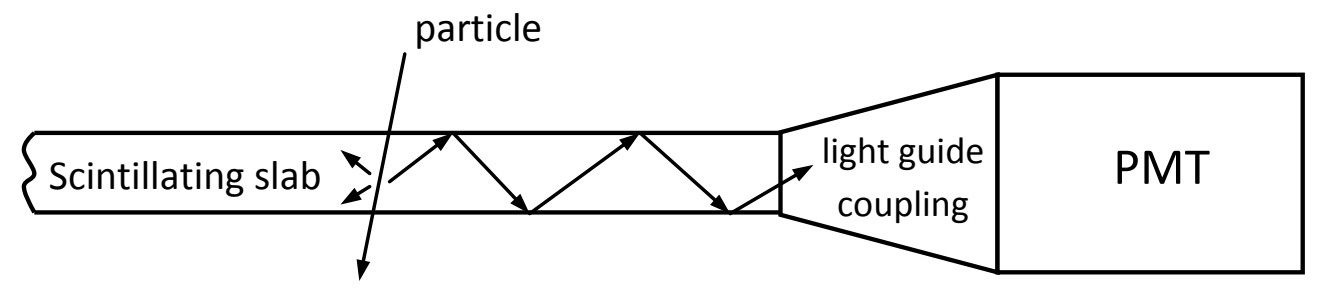

Figure 2.4.15 Basic elements of hodoscope detector.

The ENGE hodoscope system consisted of two identical layers of plastic scintillators, Bicron $\mathrm{BC} 420$. The system was designed to withstand a $5 \mathrm{MHz}$ rate in the electron arm. To keep single rates of each scintillator well below $1 \mathrm{MHz}$, the hodoscope layers were divided into 25 scintillator segments each. Each segment had a scintillator slab of $120^{\mathrm{L}} \times 40^{\mathrm{W}} \times 10^{\mathrm{T}} \mathrm{mm}^{3}$ and was equipped with acrylic light guides and Hamamatsu H6612 3/4" phototubes on both ends. A schematic picture of the hodoscope system is shown in Fig. 2.4.16. 


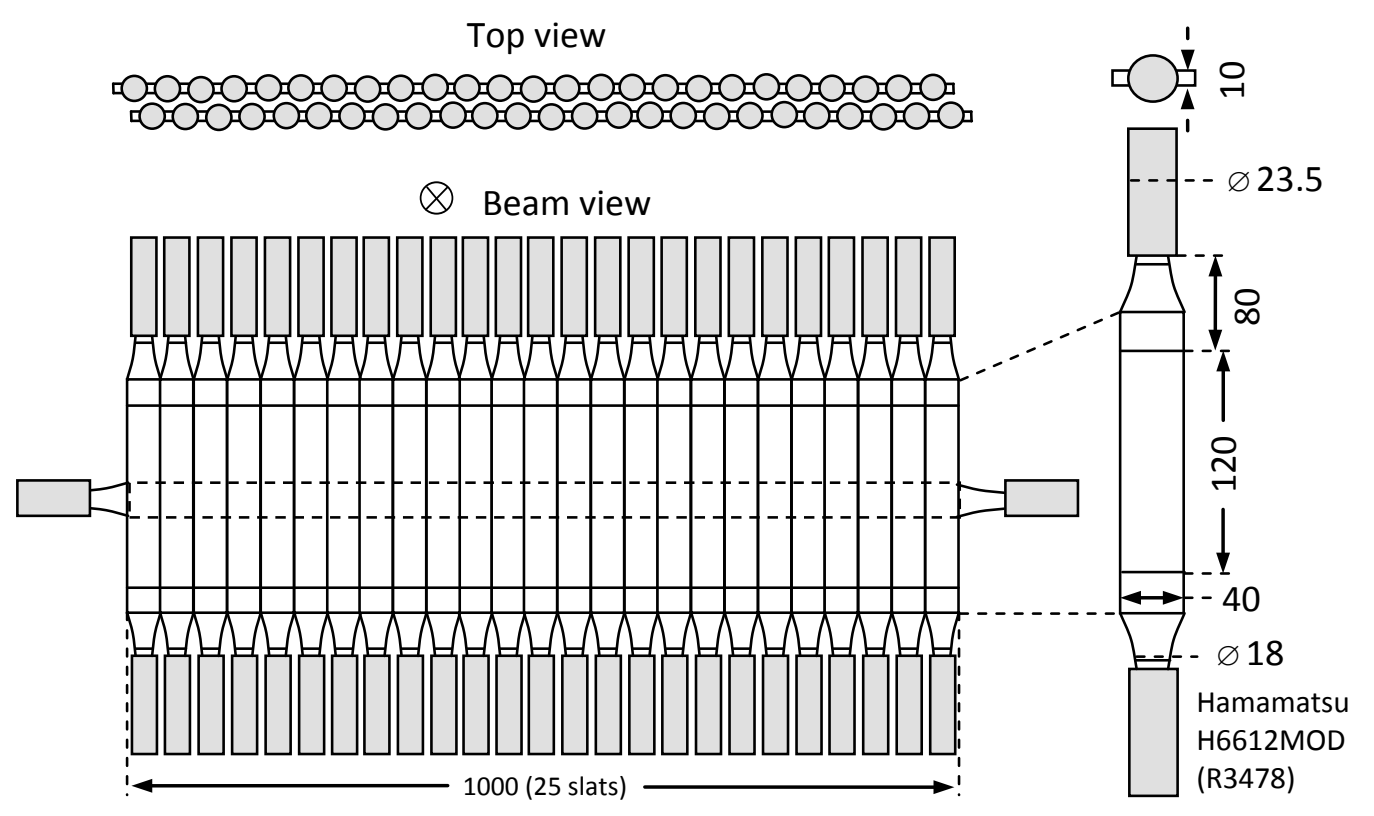

Figure 2.4.16 ENGE hodoscope system (beam's eye). (Units are given in mm).

The EHODO3 scintillator placed behind the first and second layers was only used for timing offset calibrations of the other two layers. Thus, it did not have to cover the entire acceptance. The basic parameters of the ENGE hodoscope system are listed in Table 2.4.8.

Table 2.4.8 Parameters of ENGE hodoscope system.

\begin{tabular}{ll}
\hline Enge hodoscope system & EHODO1, EHODO2, EHODO3 \\
Number of slats in the layer & EHODO1 $=25$, EHODO2 $=25$, EHODO3 = 1 \\
Plastic scintillator & Bicron BC420 \\
Geometry of scintillator slab & $120^{\mathrm{L}} \times 40^{\mathrm{W}} \times 10^{\mathrm{T}}$ \\
Number of PMTs & $25 \cdot 2+25 \cdot 2+1 \cdot 2=102$ \\
PMT & $3 / 4^{\prime \prime}$ Hamamatsu H6612MOD (R3478)
\end{tabular}

The timing signal from the hodoscope system was used for setting the coincidence trigger between the particles of interest, electron and kaon, and also was utilized to provide the time reference for the drift chamber tracking routine. The anode signals from 
each PMT were split and one copy recorded by ADCs for pulse height determination and the other copy sent to a low-threshold discriminator. The signals from the discriminator output were recorded by high resolution TDCs and also sent to the trigger logic.

\subsubsection{HKS detector package}

The HKS detector package included the following items: two drift chambers (HDC1 and HDC2) for particle tracking, three scintillator planes (1X, $1 \mathrm{Y}$ and $2 \mathrm{X})$ to measure the time of flight (TOF), and three aerogel and two water Cherenkov detectors for particle identification (PID). The basic parameters of the detector package are collected in Table 2.4.9. The geometrical positioning of the detectors is schematically shown in Fig. 2.4.17.

Table 2.4.9 HKS detector package.

\begin{tabular}{|c|c|c|c|c|}
\hline HKS & & Detectors & & Purpose \\
\hline Vacuum chamber & & - & & Extend vacuum until HDC1 \\
\hline Drift hombor & HDC1 & & HDC2 & Dontiolotrolrino \\
\hline Dritt cnambers & 6 wire planes & & 6 wire planes & Particle tracking \\
\hline Plactic cointillatorc & $1 \mathrm{X}$ & $1 Y$ & $2 X$ & Time of flight (TOF) \\
\hline F Tastic semintinators & 17 segments & 9 segments & 18 segments & calculations \\
\hline Aerogel Cherenkov & $\mathrm{AC} 1$ & $\mathrm{AC} 2$ & $\mathrm{AC} 3$ & Pion suppression and PID \\
\hline & 7 segments & 7 segments & 7 segments & \\
\hline Water Cherenkov & WC1 & & WC2 & Proton suppression and PID \\
\hline & 12 segments & & 12 segments & \\
\hline
\end{tabular}




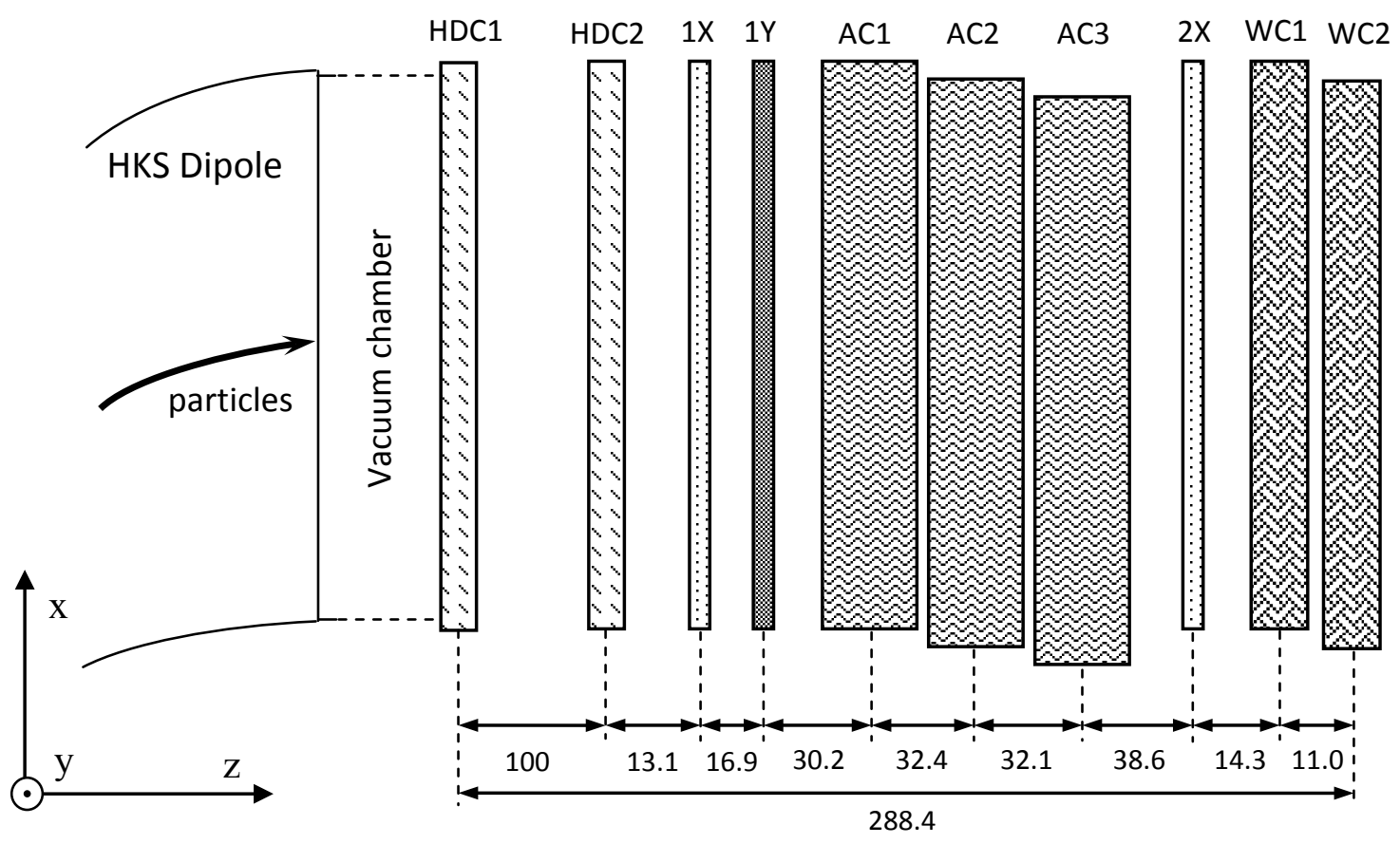

Figure 2.4.17 Schematic layout of HKS detector package. The distances are given in $\mathrm{cm}$. The figure is not to scale.

The distances in the figure are relative to the first HDC1. However, in the analysis software the nominal center of the detector package was positioned along the focal plane of the HKS spectrometer, located between $\mathrm{HDC} 1$ and $\mathrm{HDC} 2$, approximately $48 \mathrm{~cm}$ away from HDC1. Different momenta particles passing the dipole magnet focus in different spots forming a slanted focal plane (Fig. 2.4.18).

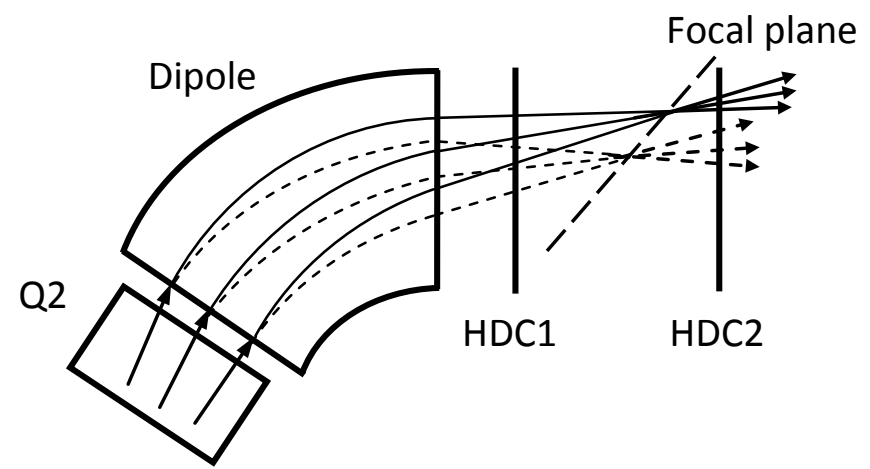

Figure 2.4.18 Trajectories of the particles with different momenta. 
A vacuum chamber is used to extend the vacuum from the exit of the dipole magnet to the first drift chamber. The vacuum prevents energy loss and multiple scattering along the particle's trajectory, which can result in a change of the initial path. All detectors were positioned perpendicularly with respect to the central momentum trajectory. The hodoscopes layers $1 \mathrm{X}, 2 \mathrm{X}$, and $1 \mathrm{Y}$ were segmented in $x$ and $y$ direction respectively. The aerogel Cherenkov detectors are used to veto pions, while two water Cherenkov detectors were utilized for proton reduction. The TOF together with AC and WC provided the particle identification. To avoid dead areas the layers of AC and WC detectors were slightly shifted with respect to each other. The descriptions of each component of the HKS detector package are given further.

\section{HKS drift chambers}

There were two identical drift chambers installed in the HKS detector rack for the purpose of particle tracking. They were designed and constructed by the Hampton University group. The conceptual design of the chamber is very similar to the standard Hall C SOS drift chambers. The chamber is made of six wire planes $u u^{\prime} x x^{\prime} v v^{\prime}$ with $u u^{\prime}$ and $v v^{\prime}$ rotated by $60^{\circ}$ clockwise and counterclockwise with respect to the $x$ axis (Fig. 2.4.19). That allows measuring twelve coordinate variables. This wire configuration not only provides two dimensional position reconstruction, but also allows resolving ambiguities caused by multiple hits. The sense wires are separated by $1 \mathrm{~cm}$, which sets the maximum drift distance to be $0.5 \mathrm{~cm}$ (Fig. 2.4.20). The effective area of each plane is $122.4 \times 30.5 \mathrm{~cm}^{2}$. Spacing between a sense wire and a field wire is $0.5 \mathrm{~cm}$. For resolving 
the left-right ambiguity, the primed wires are offset by half of the cell size with respect to the unprimed ones. The sense wires are $25 \mu \mathrm{m}$ in diameter and made of gold plated tungsten. A beryllium copper alloy was used to create the $90 \mu \mathrm{m}$ wide field wires. The cathode planes, placed between the coordinate planes, are made of a double-sided, copper-coated, Mylar foil. The sense wires were operated at zero potential, while the potential wires and cathode foils were connected to a negative high voltage. The chambers were operated with the same gas mixture as the ENGE drift chamber.

The drift chambers were mounted directly on the HKS dipole magnet, since they have to be the first detectors in the HKS arm. Since they had to measure the particles trajectories, the precise location of the chambers with respect to the magnet was critical. The particles' trajectories are measured at the HKS focal plane, located between the chambers, separated from each other by 1 meter (Fig. 2.4.18). To minimize multiple scattering of the particles along their path, a gas-tight bag filled with helium was placed between the chambers.

The signal from the anode (sense) wires was amplified and discriminated by Nanometric N277-L cards, mounted on both sides of the chamber. There were 20 cards per chamber, operated at \pm 5 Volts provided by two Acopian power supplies. Each card reads out 16 wires. If the signal is above the discriminator threshold a logic pulse is sent to a F1 multi-hit TDC. The TDC information is read out only if there is a common stop in the trigger system. According to device's specifications, the TDC can measure up to 16 hits with a resolution of $120 \mathrm{ps}$ least significant bit (lsb). After time to distance conversion, a tracking routine is used to identify particle trajectories.

The basic parameters of the HKS drift chambers are presented in the Table 2.4.10. 


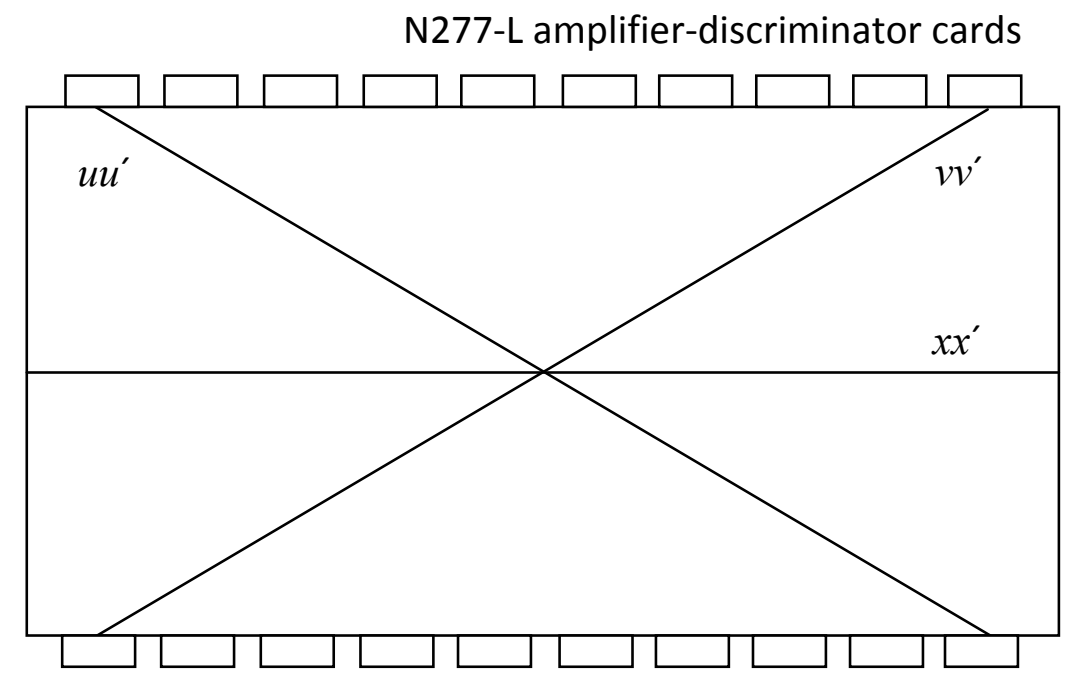

Figure 2.4.19 Wire layout of the HKS drift chamber. (beam's view).

Wire legend:

$\bigcirc$ Sense (anode) wires $(\varnothing 25 \mu \mathrm{m})$

$\bigcirc$ Field wires $(\varnothing 90 \mu \mathrm{m})$

- Cathode Cu-Mylar foils

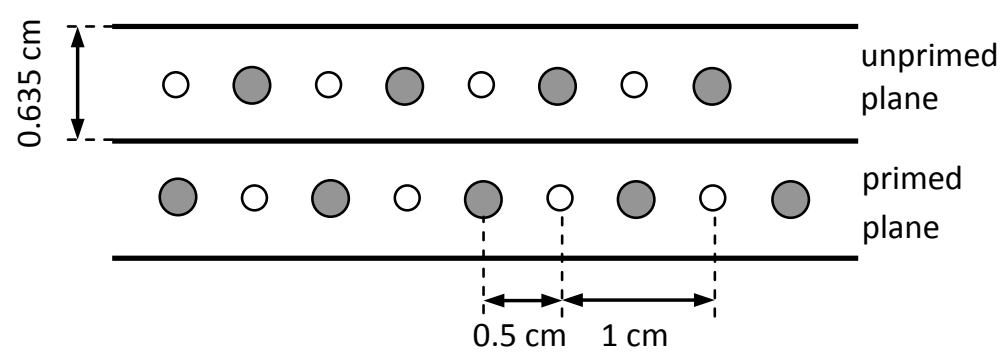

Figure 2.4.20 Layout of HKS drift chamber cell. 
Table 2.4.10 Geometrical and operational parameters of HKS drift chambers.

\begin{tabular}{ll}
\hline Geometrical parameters & \\
\hline Chamber dimension $(\mathrm{L} \times \mathrm{W} \times \mathrm{T})$ & $150.5 \times 57.8 \times 7.6 \mathrm{~cm}^{3}$ \\
Effective area & $122.4 \times 30.5 \mathrm{~cm}^{2}$ \\
Wire plane configuration & $u u^{\prime}\left(+60^{0}\right) x x^{\prime} v v^{\prime}\left(-60^{0}\right)$ \\
Spacing between sense wires & $1 \mathrm{~cm}$ \\
Spacing between sense and potential wires & $0.5 \mathrm{~cm}$ \\
Gap between two foils & $0.635 \mathrm{~cm}$ \\
\hline Operational Parameters & \\
\hline High Voltage & $-1970 \mathrm{~V}$ \\
Threshold & $3.0 \mathrm{~V}$ \\
Gas content & Argon - Ethane $(50 / 50)$, plus $1 \%$ alcohol \\
Gas pressure & $\sim 16 \mathrm{psi}$ \\
Read-out card & Nanometric N277-L (amplifier-discriminator) \\
Number of cards per chamber & 20 \\
\hline
\end{tabular}

\section{HKS hodoscope system}

Three plastic scintillator planes HTOF1X (1X), HTOF1Y (1Y), and HTOF2X (2X) comprise the HKS hodoscope system. The scintillators used in the planes are Bicron BC408. The geometrical placement of the planes is shown on the HKS spectrometer diagram (Fig. 2.4.17). The first plane, 1X, is positioned right after HDC2. Then it is followed by the $1 \mathrm{Y}$ plane located $16.9 \mathrm{~cm}$ further along the beam direction. The second $x$-plane, $2 \mathrm{X}$, is placed approximately 1.502 meters away from the first $1 \mathrm{X}$ layer. The scintillators use Hamamatsu H1949-50 photomultiplier tubes, with two PMTs per bar. The tubes are operated at -1800 Volts. The layers $1 \mathrm{X}, 1 \mathrm{Y}$, and $2 \mathrm{X}$ have 17,9 , and 18 scintillator bars, respectively. The schematic of the $1 \mathrm{X}$ layer is shown on the Fig. 2.4.21. The geometrical size of a scintillator bar is $300^{\mathrm{L}} \times 75^{\mathrm{W}} \times 20^{\mathrm{T}} \mathrm{mm}^{3}$. The layer covers the effective area of $125^{\mathrm{W}} \times 30^{\mathrm{H}} \mathrm{cm}^{2}$. The $2 \mathrm{X}$ layer, similarly to $1 \mathrm{X}$, consists of 18 
scintillator bars with a size of $400^{\mathrm{L}} \times 75^{\mathrm{W}} \times 20^{\mathrm{T}} \mathrm{mm}^{3}$. The $1 \mathrm{Y}$ layer is positioned perpendicularly with respect to the $x$-planes. Each bar in the $1 \mathrm{Y}$ layer is $35 \mathrm{~mm}$ wide, $1250 \mathrm{~mm}$ long, and $20 \mathrm{~mm}$ thick. The layout of the 1Y layer is shown in Fig. 2.4.22. Since the width of the scintillator bar in this panel is smaller than the diameter of the PMT, the light guides had to be bent to the side in order to leave no gaps between the scintillator bars. The parameters of the HKS hodoscope system are listed in the Table 2.4.11.

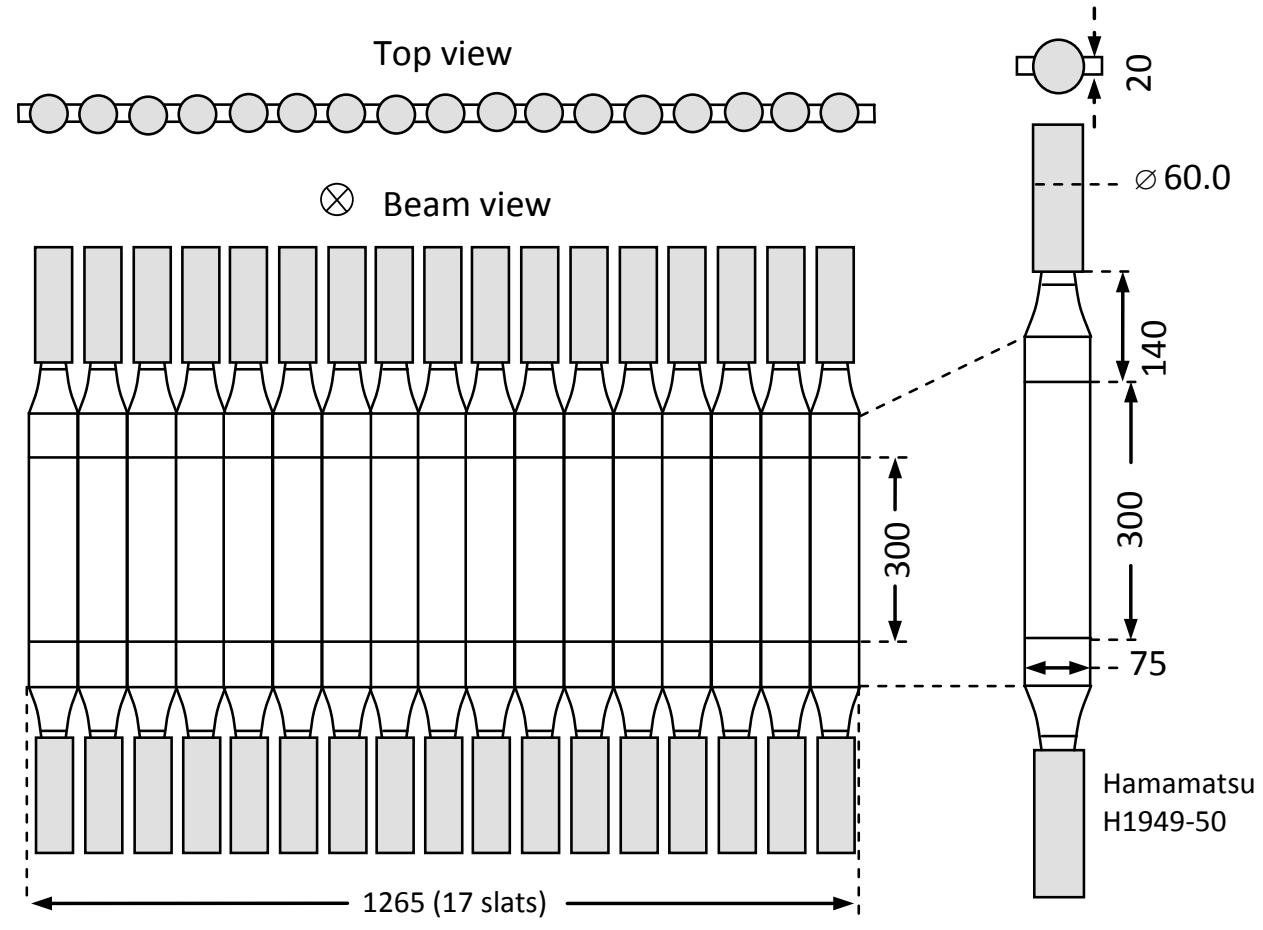

Figure 2.4.21 Schematic of the $1 \mathrm{X}$ (Bicron BC408) layer. (The units are in $\mathrm{mm}$ ). 

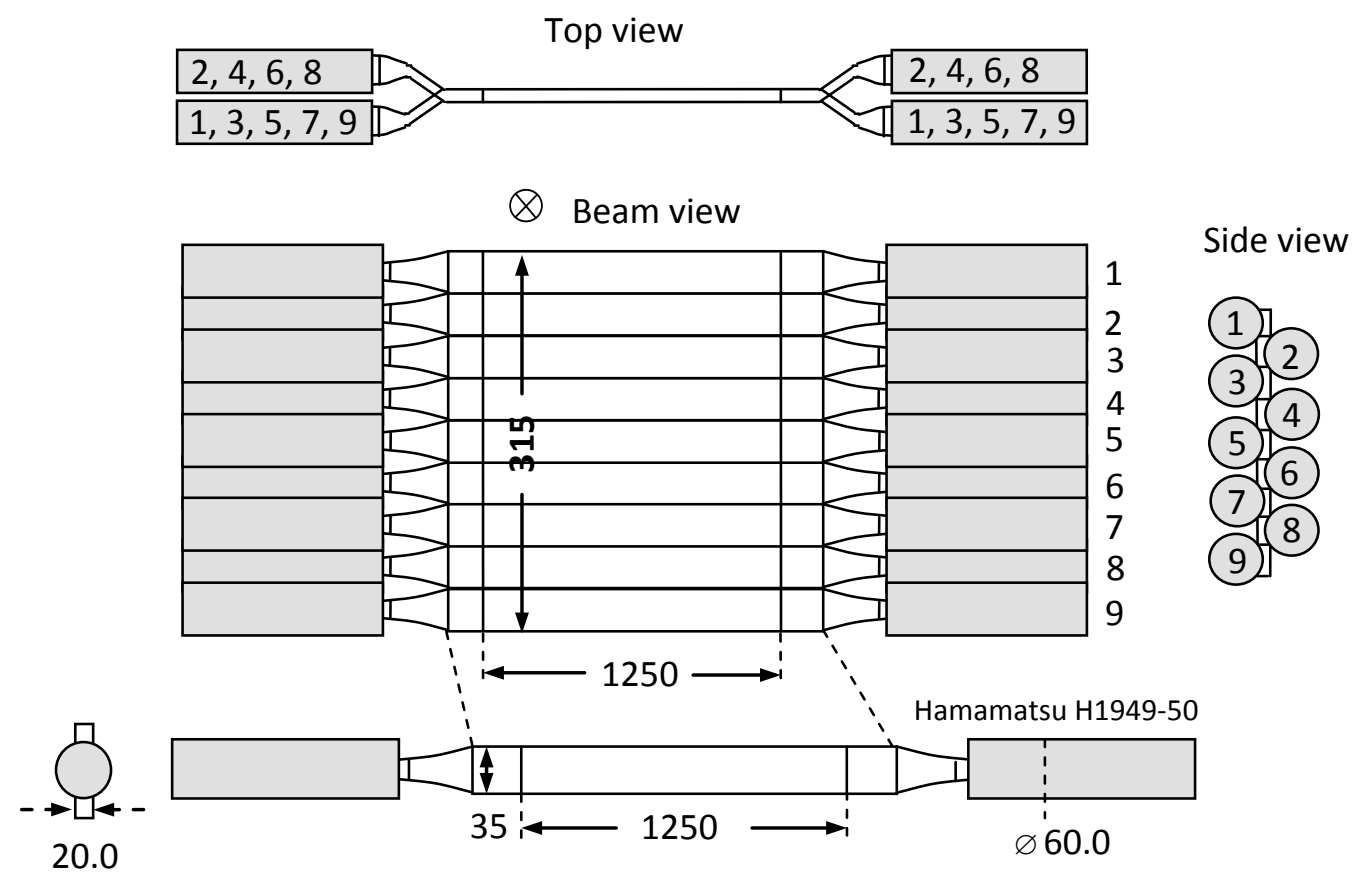

Figure 2.4.22 Schematic of the 1Y (Bicron BC408) layer. (The units are in mm).

Table 2.4.11 Parameters of the HKS hodoscope system.

\begin{tabular}{ll}
\hline HKS Hodoscope system & HTOF1X $(1 X)$, HTOF2Y (1Y), HTOF2X (2X) \\
Effective area & $125^{\mathrm{W}} \times 30^{\mathrm{H}} \mathrm{cm}^{2}$ \\
Photomultiplier tubes & Hamamatsu H1949-50 \\
Diameter of PMT & $60 \mathrm{~mm}$ \\
Operational High Voltage & $-1800 \mathrm{~V}$ \\
\hline Hodoscope layer 1X & 17 scintillator bars \\
Scintillator slat dimensions & $300^{\mathrm{L}} \times 75^{\mathrm{W}} \times 20^{\mathrm{T}} \mathrm{mm}^{3}$ \\
\hline Hodoscope layer 1Y & 9 scintillator bars \\
Scintillator slat dimensions & $1250^{\mathrm{L}} \times 35^{\mathrm{W}} \times 20^{\mathrm{T}} \mathrm{mm}^{3}$ \\
\hline Hodoscope layer 2X & $18 \mathrm{scintillator} \mathrm{bars}$ \\
Scintillator slat dimensions & $400^{\mathrm{L}} \times 75^{\mathrm{W}} \times 20^{\mathrm{T}} \mathrm{mm}^{3}$ \\
\hline
\end{tabular}




\section{Aerogel Cherenkov detectors}

The aerogel Cherenkov (AC) detectors, placed behind hodoscope layer 1Y, were used to separate pions from kaons in the momentum range $1.05-1.35 \mathrm{GeV} / \mathrm{c},\left(p_{0}=1.2 \pm 0.15\right.$ $\mathrm{GeV} / \mathrm{c})$. The $\mathrm{AC}$ detector set consisted of three identical threshold type Cherenkov

detectors: AC1, AC2, AC3, positioned one behind another facing the beam. Each AC layer included 7 independent segments, with two photomultiplier tubes per segment. FIU was responsible for construction and operation of these detectors. The working principles, parameters, and performance of the aerogel Cherenkov detectors will be described in detail in Chapter 3.

\section{Water Cherenkov detectors}

To separate kaons and protons, another type of Cherenkov counters were installed behind the second wall of plastic scintillators, HTOF2X. Because of a relatively large operational current, $\sim 30 \mu \mathrm{A}$, the hadron rates in E01-011 experiment were estimated to be roughly three times higher than the rates in the previous HNSS experiments. That became a key motivation for usage of water Cherenkov detectors instead of the plastic (Lucite) Cherenkov counters employed earlier. Two identical layers of water Cherenkov counters, WC1, and WC2, used in a kaon trigger allowed proton suppression by a factor of $10^{-4}$. Each layer contained 12 segments, with an effective Cherenkov radiator volume $350^{\mathrm{L}} \times 150^{\mathrm{W}} \times 75^{\mathrm{T}} \mathrm{mm}^{3}$ per segment.

The counters were developed and tested in Japan by the Tohoku University group [54]. Pure water was initially expected to be used as a Cherenkov radiator. The test at 
KEK of the first WC counter prototype showed that the addition of a wavelength shifter (Amino-G-salt) allowed increased rejection power. The separation of the proton and kaon distributions significantly improved when mixing pure water with Amino-G-salt acid (Fig. 2.4.23).

The wave length shifter, amino-G-salt mixture, is composed of 2-amino-6 and 8naphthalene-disulfonic acid added to pure water in $50 \mathrm{mg}$ per liter proportion. The chemical water was contained in a 3-mm-thick white acrylic diffusion box, with two 5 cm PMTs attached on the opposite sides of the container. The acrylic material, Mitsubishi Acrylite \# 402, acted as a diffuse reflector and provided at maximum $94.8 \%$ reflectance for light of $458.5 \mathrm{~nm}$ wavelength. Since the container had to be a leak proof, the signal collecting PMTs had to be connected through special transparent windows, made of 3 mm thick acrylic material (Mitsubishi Acrylite \# 000). The window transmittance in the effective wavelength range of the PMT was estimated to be $90 \%$ [59]. 

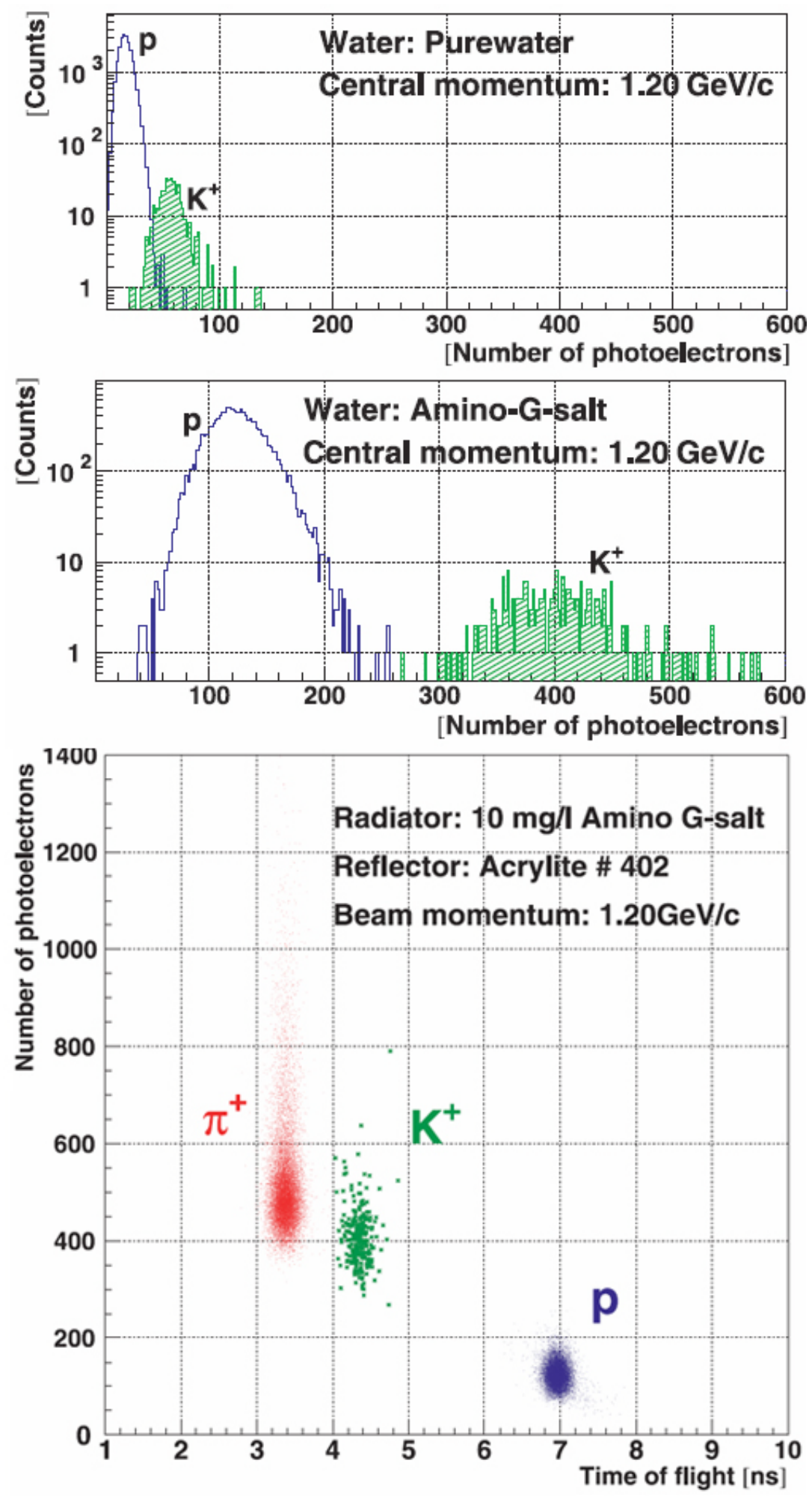

Figure 2.4.23 Results from KEK test of water Cherenkov detectors with pure water and chemical water radiator. The increased separation between kaons and protons allows more efficient proton rejection, [54]. 


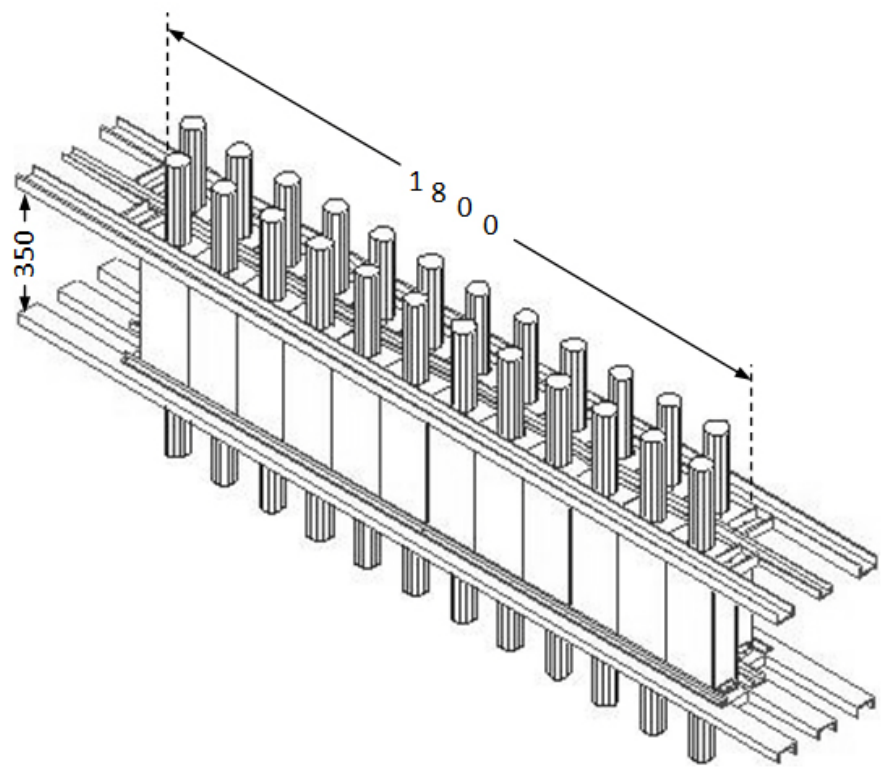

Figure 2.4.24 Schematic drawing of the water Cherenkov counters [56]. (The units are in $\mathrm{mm}$ ).

A schematic drawing of the water Cherenkov detectors is shown in Fig. 2.4.24. An actual photograph of the WC counters mounted on the detector rack is presented in Fig. 2.4.25. The basic parameters of the counters are shown in Table 2.4.12.

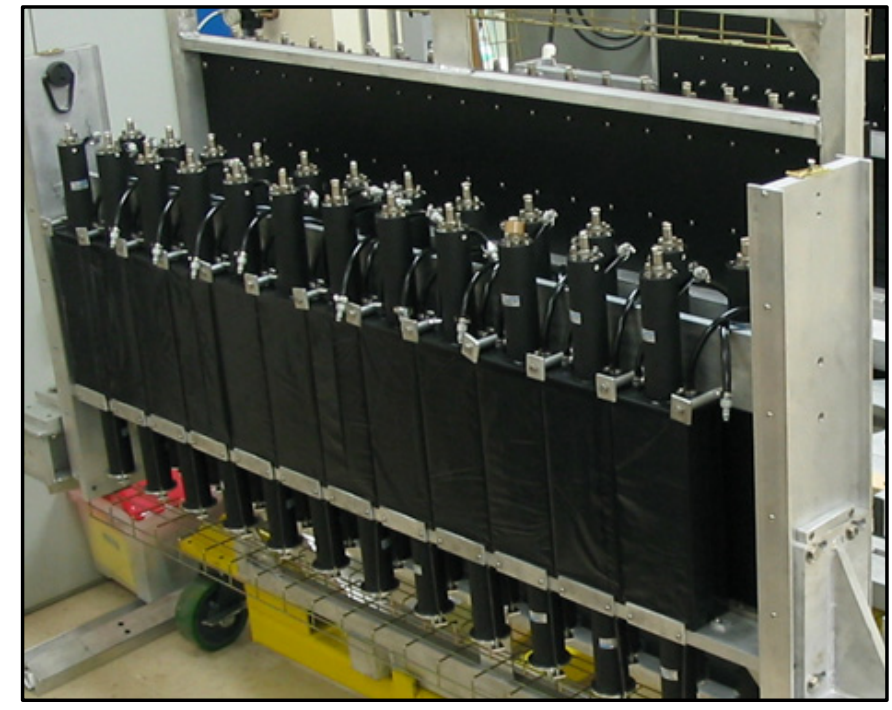

Figure 2.4.25 Photograph of the water Cherenkov counters mounted on the HKS detector rack [56]. 
Table 2.4.12 Water Cherenkov detector parameters.

\begin{tabular}{ll}
\hline Water Cherenkov detector pack & WC1 (12 segments), WC2 (12 segments) \\
Radiator Volume & $350^{\mathrm{L}} \times 150^{\mathrm{W}} \times 75^{\mathrm{T}} \mathrm{mm}^{3}$ \\
Container & Volume $=4$ liters $;$ Weight $=5 \mathrm{~kg}$ \\
Diffusion box material & Mitsubishi Acrylite $\# 402 ; 3 \mathrm{~mm}$ thick \\
& Reflectance $=93 \% ;$ Transmittance $=7 \%$ \\
PMT window material & Mitsubishi Acrylite \#000;3 mm thick \\
& Transparent \\
Photomultiplier tube & Hamamatsu R329-02; Two per segment \\
PMT effective area (diameter) & $5 \mathrm{~cm}$ \\
\hline
\end{tabular}

\subsection{Electronics and data acquisition}

\subsubsection{Trigger}

As we already mentioned there are two spectrometer arms involved in the E01-011 experimental setup. The electron arm measures scattered electrons with the help of EDC and EHODO planes. The hadron arm serves for tracking the positively charged products of the reaction, which are protons, kaons, and pions. The HKS detector package in the hadron arm allows particle identification, while PID was not necessary in ENGE. Because of the high rate in the kaon arm, a coincidence trigger had to be employed. The coincidence between the electron and kaon was set when single arm triggers were within a window of approximately $50 \mathrm{~ns}$.

The hadron trigger is composed of the combination of the following detectors: $1 \mathrm{X}$ and $2 \mathrm{X}$ hodoscope layers, $\mathrm{AC}$ and WC detectors. The scintillator plates are sensitive to all three particles traversing the planes. The AC counters only fire when pions pass through, and are blind for the rest of the particles. The WC counters will detect all three 
particles. Introducing the thresholds in the ADC (analog-to-digital converter) electronic units, one can regulate the detection level of the particles of interest. In case of the $1 \mathrm{X}$ and $2 \mathrm{X}$ hodoscopes the signal from all three particles was measured, and the unbiased trigger (1X \& 2X) was created. The AC detectors used a threshold to cut out both protons and kaons, located in the pedestal area, and to collect the pions only. In the trigger the signal from $\mathrm{AC}$ was taken as veto, $\overline{\mathrm{AC}}$. The WC counters were able to separate kaons and protons, and were detecting kaons above an adjustable threshold.

Employing the described detector signals one can set the following pretriggers:

1) Kaon pretrigger: $1 X \& 2 X \& \overline{\mathrm{AC}} \& \mathrm{WC}$

2) Proton pretrigger: $1 \mathrm{X} \& 2 \mathrm{X} \& \overline{(\mathrm{AC} \text { or } \mathrm{WC})}$

3) Pion pretrigger: $1 X \& 2 X \& A C$

By AC, we understand the sum of the aerogel Cherenkov planes that can be arranged in either two out of three, or three out of three. The WC means the sum of the coincidence on both water Cherenkov layers.

The proton and pion pretriggers were prescaled with the help of a dynamic scaling circuit. Such a circuit uses the gate time width, called scale factor, to accept or reject the signal. Within a set gate width it accepts only one pretrigger in a time. The prescaling is done with the purpose of maintaining manageable data rates. Without the prescaling procedure the DAQ system would not be able to handle the high detection rates resulting in large dead times. A small sample of rejected triggers was collected for efficiency studies. 
The prescaled pion and proton triggers combined with the logical ".or." of the kaon trigger form the HKS trigger. Signals from each stage of the logic were sent to scalers and low-resolution TDC units for efficiency studies. The information about computer dead time was provided by scalers.

In order to reduce accidental kaon overkill in the trigger, a segmentation of the detectors in six groups was used. From Monte Carlo simulations it was found that particles from different momentum regions lead to characteristic hit patterns on the detectors (Fig. 2.5.1). This means that for a specific limited momentum range only few segments of each detector plane are active. The groups were assigned in such a way that the segmentation follows the trajectories of the particles of different momentum regions. The layout of group segmentation is shown in Fig. 2.5.2.

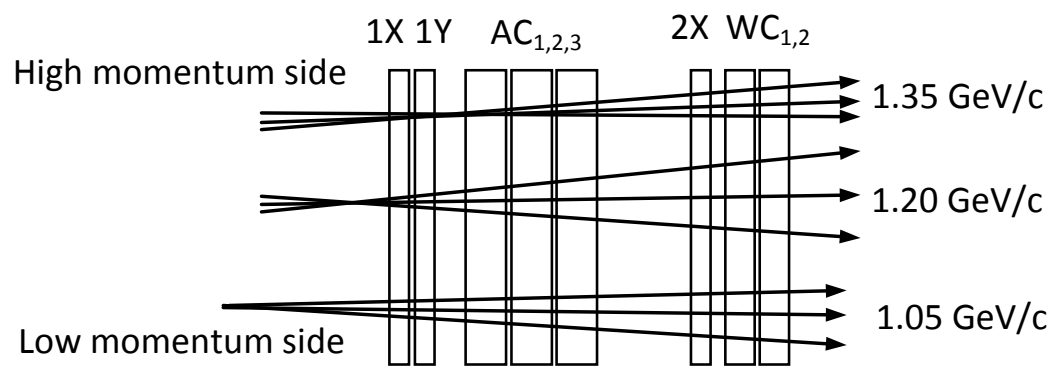

Figure 2.5.1 Particles with different momentum focus in different points. 


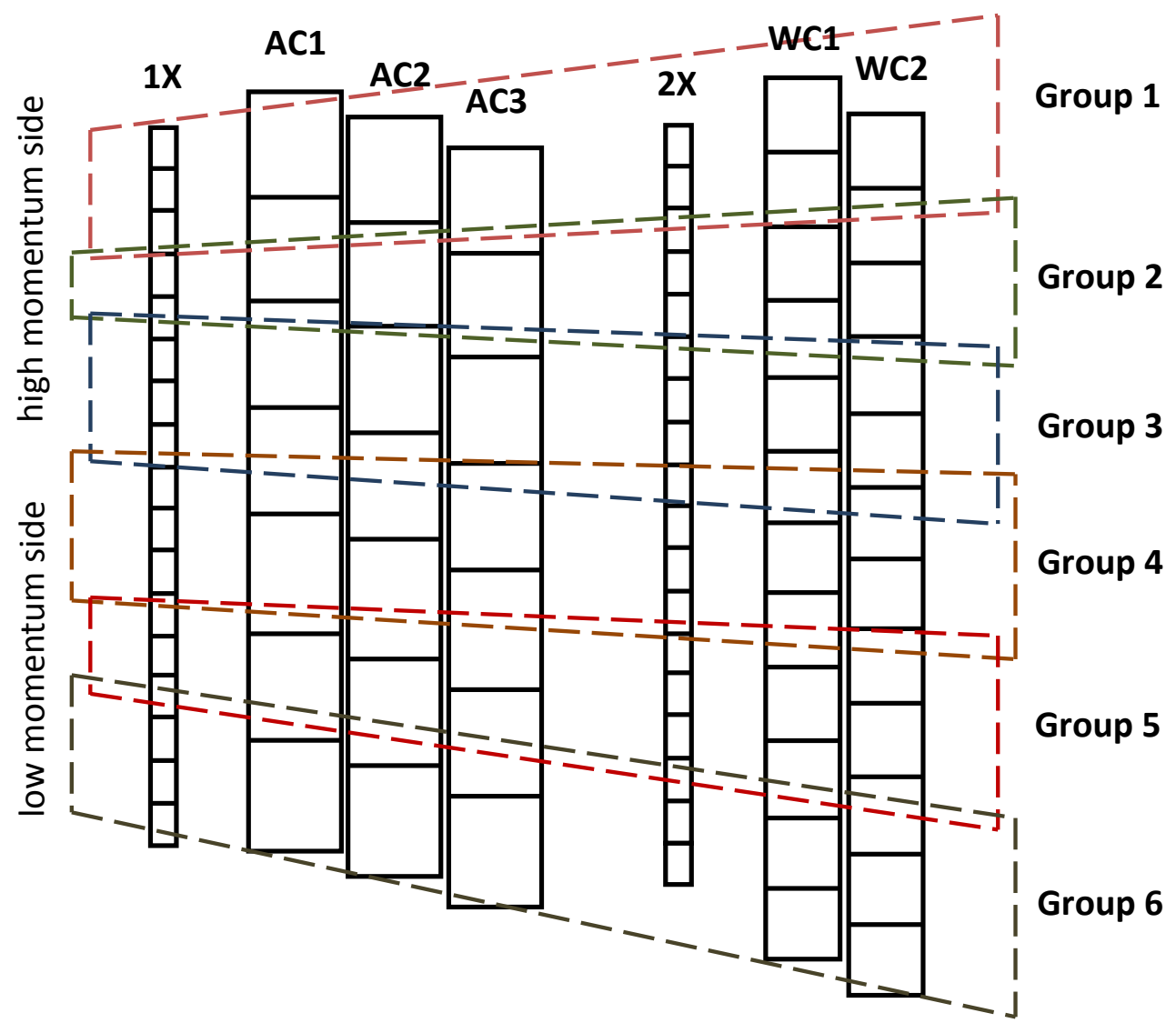

Figure 2.5.2 Schematics of the HKS trigger grouping.

The grouping is done with the help of TUL (Tohoku Universal Logic) modules, developed at Tohoku University, Japan. The TUL is a programmable logic module, which consists of various numbers of logic gates and flip-flop elements. In total six TUL8040 modules were utilized to group the signals from the following six counters: HTOF1X, HTOF1Y, HTOF2X, AC, WC, EHODO. To easily rearrange the combination of groups, the TUL module was built on the base of logic module Altera APEX$20 \mathrm{~K} 300 \mathrm{E}$, which allowed the logic to be reprogrammed without any change of cabling. 


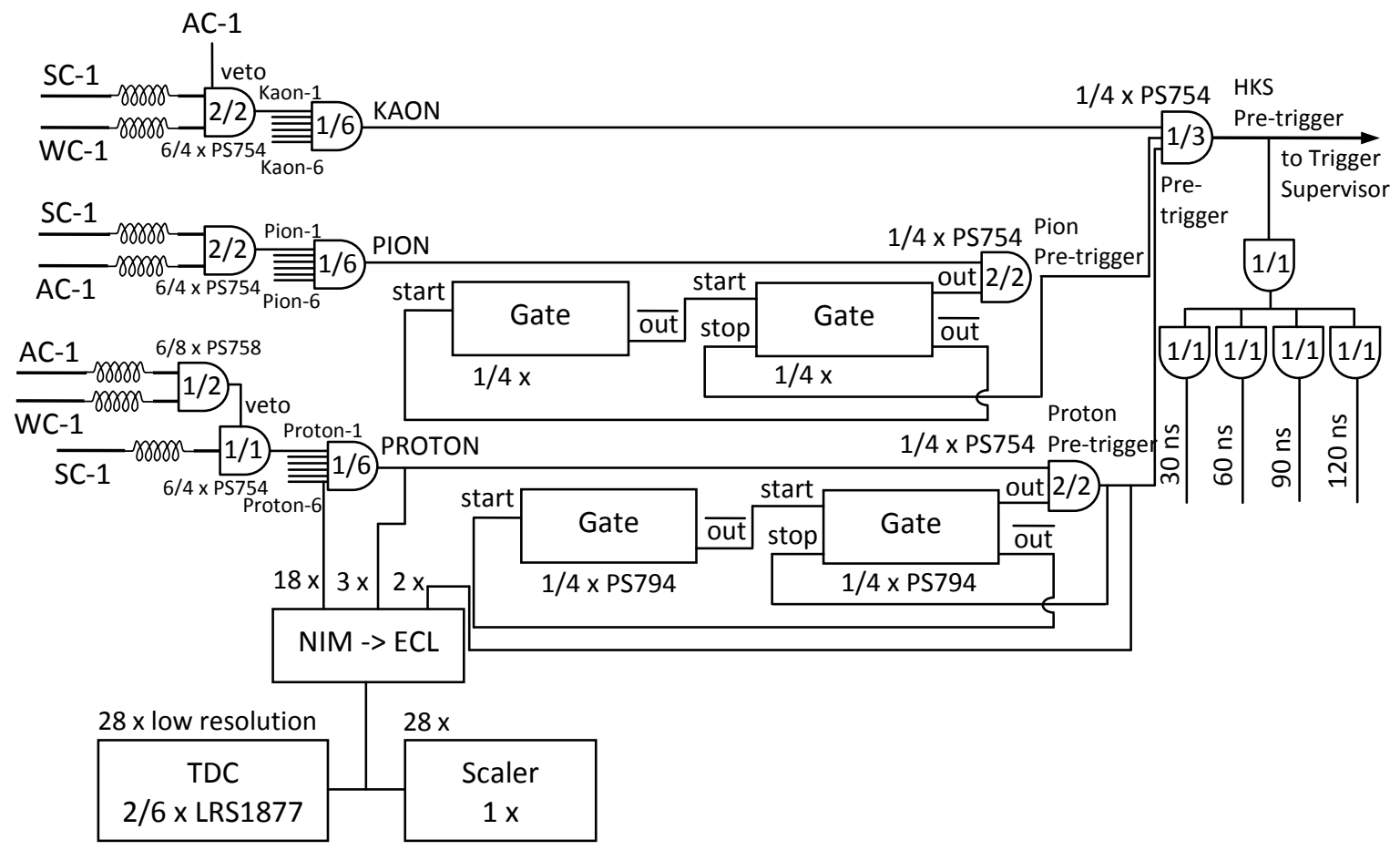

Figure 2.5.3 Schematics of hadron trigger.

The diagram of the logic for the hadron trigger is shown in Fig. 2.5.3 [60]. The timing in the trigger is determined by the two hodoscope layers HTOF1X and HTOF2X. The signals from scintillators are collected in an AND (i.e. HTOF1X AND HTOF2X) to set the pre-trigger. The PID pre-triggers are created from one of the six grouped signals measured from AC, WC, and HTOF counters. Furthermore, the pretrigger signals from each spectrometer are sent to a LeCroy 8LM programmable logic module (Fig. 2.5.4) $[60]$. 


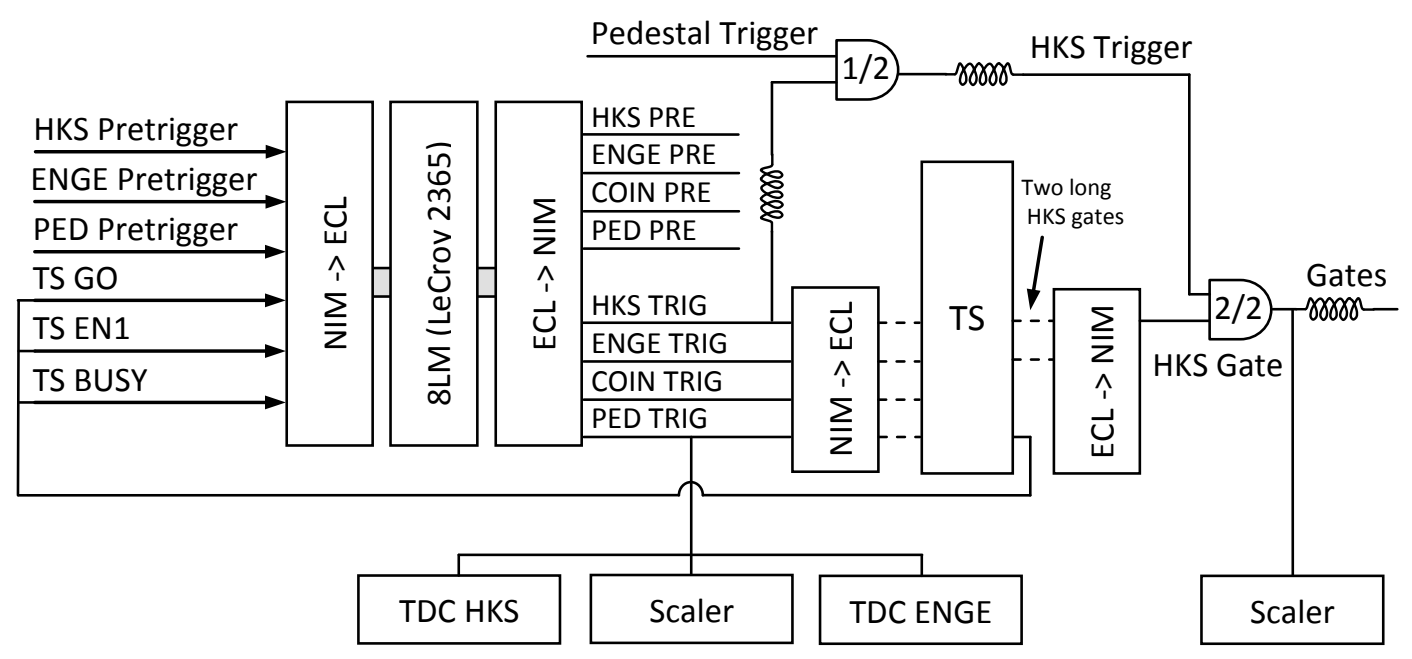

Figure 2.5.4 Trigger Supervisor (TS).

The 8LM (LeCroy 2365) module, developed and built at Jefferson lab, is programmed to classify an event as either an HKS single-arm event, an ENGE single-arm event, or a coincidence event, depending on the timing of the inputs. In addition to pretrigger signals the 8LM unit receives signals, from the trigger supervisor (TS): TS GO, TS EN1 and TS BUSY. The meanings of the 8LM input and output signals are explained in Table 2.5.1.

After the 8LM the HKS TRIG signal is split: one part is delayed and ANDed with the pedestal trigger, while the other is sent to the TS. The TS produces a pair of long gates ( $>100 \mathrm{~ns}$ ), which further form an AND with delayed HKS Trigger. After an AND is formed the ADC and TDC gates are generated. 
Table 2.5.1 LeCroy 8LM input and output signals.

\begin{tabular}{|c|c|c|}
\hline \multicolumn{2}{|c|}{ INPUT } & OUTPUT \\
\hline \multicolumn{2}{|l|}{$(\mathrm{HKS}) \&(\mathrm{EN} 1)$} & HKS PRETRIG \\
\hline \multicolumn{2}{|l|}{$(\mathrm{ENGE}) \&(\mathrm{EN} 1)$} & ENGE PRETRIG \\
\hline \multicolumn{2}{|c|}{$(\mathrm{HKS}) \&(\mathrm{ENGE}) \&(\mathrm{EN} 1)$} & COIN PRETRIG \\
\hline \multicolumn{2}{|c|}{$(\mathrm{PED}) \&(\mathrm{GO}) \&(\mathrm{NOT}$ EN1) } & PED PRETRIG \\
\hline \multicolumn{2}{|c|}{$(\mathrm{HKS}) \&(\mathrm{EN} 1) \&(\mathrm{NOT}$ BUSY) } & HKS TRIG \\
\hline \multicolumn{2}{|c|}{ (ENGE) \& (EN1) \& (NOT BUSY) } & ENGE TRIG \\
\hline \multicolumn{2}{|c|}{$(\mathrm{HKS}) \&(\mathrm{ENGE}) \&(\mathrm{EN} 1) \&(\mathrm{NOT} B U S Y)$} & COIN TRIG \\
\hline \multicolumn{2}{|c|}{ (PED) \& (GO) \& (NOT EN1) \& (NOT BUSY) } & PED TRIG \\
\hline \multicolumn{3}{|l|}{ TS programming } \\
\hline $\begin{array}{l}\text { GO } \\
\text { EN1 } \\
\text { BUSY / NOT BUSY }\end{array}$ & $\begin{array}{l}\text { Run has been started. } \\
\text { Run is in progress an } \\
\text { Availability indicator } \\
\text { BUSY: Run has been } \\
\text { NOT BUSY: Run has }\end{array}$ & $\begin{array}{l}\text { data taking is enabled. } \\
\mathrm{f} \text { the TS module. } \\
\text { tarted, but data taking is disabled. } \\
\text { een started and data taking is enabled. }\end{array}$ \\
\hline
\end{tabular}

\subsubsection{Read-out electronics}

The electronics used in E01-011 experiments utilized not only the standard experimental nuclear physics electronics equipment, such as NIM, VME and Fastbus crates, CAEN and Acopian power supplies, LeCroy ADCs and TDCs, but it also employed a newly introduced F1 multihit TDC and TUL-8040 modules. The VME interfaced F1 multihit TDC was designed at Jefferson Lab as an alternative to Fastbus-based, high-resolution TDCs. The basic properties of the F1 TDC module are displayed in Table 2.5.2 [61].

Table 2.5.2 Properties of F1 multihit TDC module (in normal and high resolution modes).

\begin{tabular}{|l|l|}
\hline Normal resolution regime & High resolution regime \\
\hline 64 channels & 32 channels \\
\hline Resolution $=86.2 \mathrm{ps}(\mathrm{rms})$ or $120 \mathrm{ps}(\mathrm{lsb})$ & Resolution $=61.2 \mathrm{ps}(\mathrm{rms})$ or $60 \mathrm{ps}(\mathrm{lsb})$ \\
\hline Buffers 32 hits per channel & Buffers 16 hits per channel \\
\hline
\end{tabular}


The F1 TDC modules were used for the HKS and ENGE drift chambers in low resolution mode, and for the EHODO in high resolution mode. High resolution Fastbus LeCroy 1877 TDCs, 25 ps (1sb), were utilized for HKS hodoscope system. The detectors PMT signals were recorded with 64-channel Fastbus ADC (LeCroy 1881) and high resolution Fastbus TDC (LeCroy 1872A). The TUL-8040 modules were used for HKS detector grouping and forming of the Hadron PID trigger. Several Acopian power supplies were installed in the Hall $\mathrm{C}$ to operate the HKS and ENGE drift chambers. The rest of the detectors were powered by the CAEN power crates which were installed upstairs, in the counting house. The Table 2.5.3 summarizes the read-out electronics modules needed for each detector in E01-011 experiment [62].

Table 2.5.3 Detector read-out electronics.

\begin{tabular}{lllccc}
\hline Detector & Abbreviation & Read-out method & HR TDC & TDC & ADC \\
\hline HKS detectors & & & - & 660 & - \\
\hline Drift chamber 1 & HDC1 & Nanometric N227L & - & 720 & - \\
Drift chamber 2 & HDC2 & Nanometric N227L & 34 & - & 34 \\
TOF hodoscope (X) & HTOF1X & PMT H1949 & 18 & - & 18 \\
TOF hodoscope (Y) & HTOF1Y & PMT H1949 & 14 & - & 14 \\
Aerogel Cherenkov 1 & AC1 & PMT R1250 & 14 & - & 14 \\
Aerogel Cherenkov 2 & AC2 & PMT XP4572B/D1 & 14 & - & 14 \\
Aerogel Cherenkov 3 & AC3 & PMT XP4572B/D1 & 36 & - & 36 \\
TOF hodoscope (X) & HTOF2X & PMT H1949 & 24 & - & 24 \\
Water Cherenkov 1 & WC1 & PMT H7195 & 24 & - & 24 \\
Water Cherenkov 2 & WC2 & PMT H7195 & 178 & 1380 & 178 \\
\hline HKS sub total & & & & - & 1120 \\
\hline ENGE detectors & & & 50 & - & 50 \\
\hline Honeycomb drift chamber & EDC & Nanometric N227L & 50 & - & 50 \\
TOF hodoscope (X) & EHODO1 & PMT H6612 & 2 & - \\
TOF hodoscope (Y) & EHODO2 & PMT H6612 & & 2 \\
TOF hodoscope (Y) & EHODO3 & PMT H1949 & 280 & 2500 & 300 \\
\hline ENGE sub total & & & 1120 & 102 \\
\hline Fast raster & & & & 4 \\
BPM (Beam Position Monitors) & & & & 16 \\
\hline Grand Total & & & & \\
\hline
\end{tabular}




\subsubsection{Data acquisition system}

The E01-011 experiment utilized the standard Hall C CEBAF Online Data Acquisition (CODA) system. In total, six Read-Out Controllers (ROCs) were utilized, with two of them installed down in Hall C and the rest mounted in Hall C Electronics Control Room. For each run three types of events were recorded: 1) detector information from ADCs and TDCs, 2) Scaler information, 3) EPICS (Experimental Physics and Industrial Control System) events that contain such information as beam current, raster, magnet settings and spectrometer parameters. When a run is started the first thousand pedestal events are generated by the PED pretrigger and recorded by the DAQ. After that the DAQ records events based on the signal from the main trigger that is set by the combination of the PID pretrigger logic. The schematic diagram of the CODA system is shown in Fig. 2.5.5.

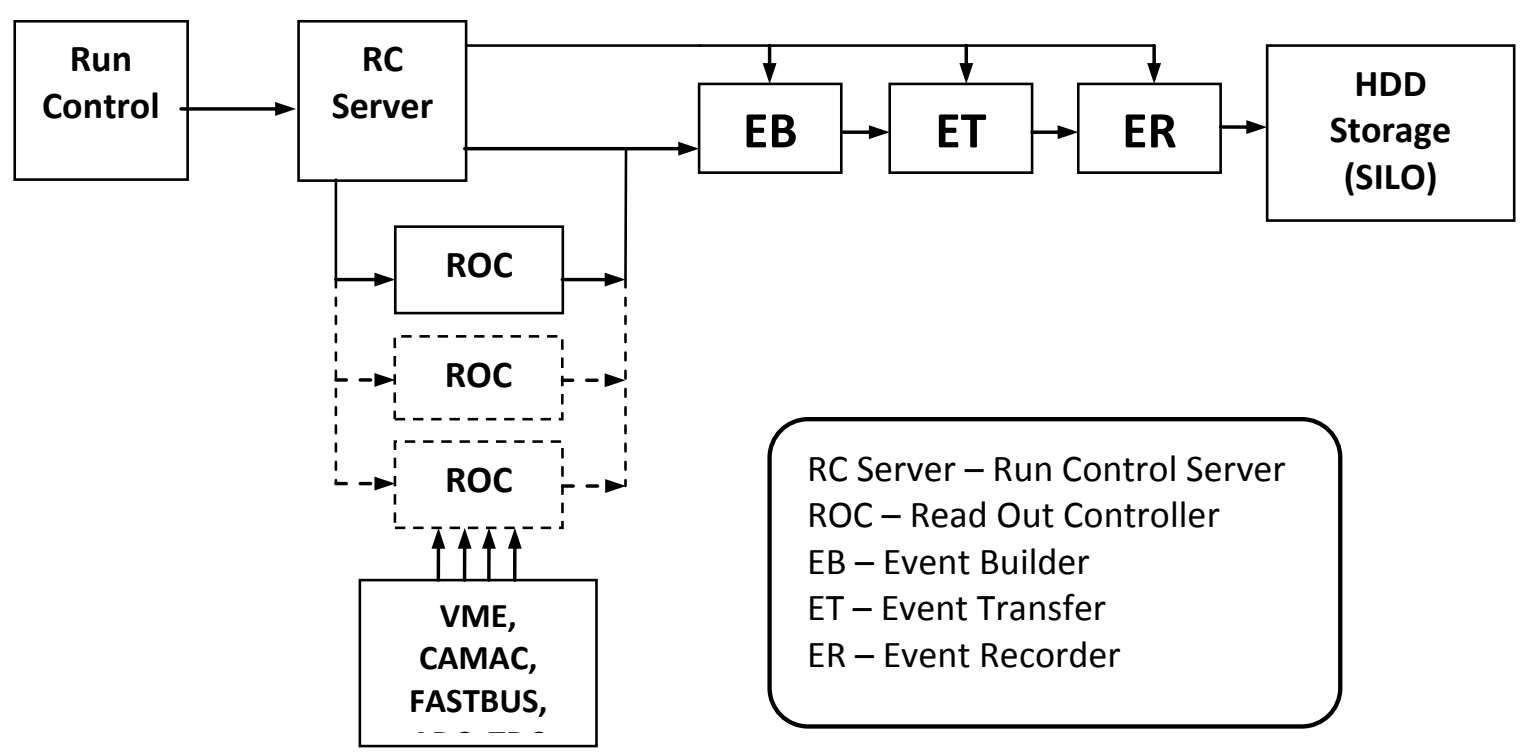

Figure 2.5.5 Schematic diagram of CODA system. 
All read-out electronics were connected to VME, CAMAC, and FASTBUS crates. Each create is managed by a separate ROC. The ROCs are read out by CODA software through the Run Control (RC) Server. The RC server serves as a central system that performs the following key processes: 1) collects and prescales triggers, 2) generates BUSY signals while processing the trigger, 3) coordinate the multilevel triggers, 4) communicates the trigger information to the ROCs, 5) administer the jobs on peripheral event processing systems.

The information from ADCs and TDCs is read out once per event, while the scalers are read out every two seconds. The read out interval for EPICS events is usually 2 - 30 seconds. Data streams from the ROCs are collected by an Event Builder (EB), which does event synchronization. The assembled events are sent to the Event Recorder (ER) by a process called Event Transfer (ET). The ER organizes the data fragments into standardized output and records it onto a hard drive. The recorded file could reach, but not exceed, the size of $1-2$ Gigabytes. Detailed information about the CODA system can be found in references $[63 ; 64]$. 


\section{CHAPTER 3}

\section{AEROGEL CHERENKOV DETECTORS}

\subsection{Cherenkov radiation principles}

The emission of Cherenkov electromagnetic radiation occurs when the velocity of a relativistic charged particle passing through dielectric material exceeds the phase velocity of the light inside of the medium. The charged particle traversing the medium polarizes the atoms in the vicinity of its track. Excited atoms return back to their ground state by emitting prompt radiation. The Cherenkov light is emitted almost instantaneously with passage of the particle. The radiation propagates in a conical coherent wavefront with the constant characteristic angle $\theta_{c}$ with respect to the direction of the charged particle [65] (Fig. 3.1.1.a).

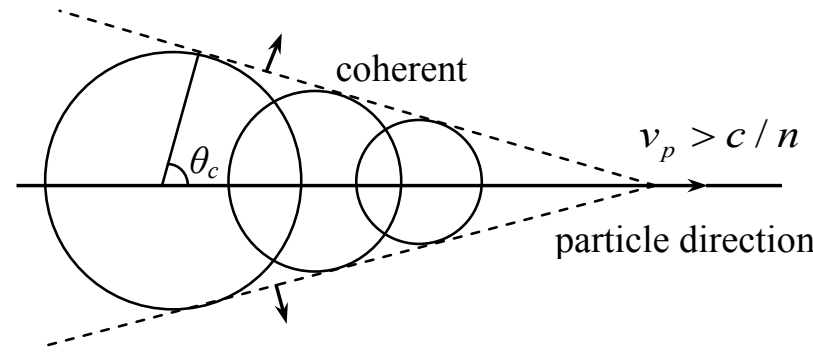

Figure 3.1.1.a Cherenkov wave propagation with coherent wavefront with a satisfied threshold contrition.

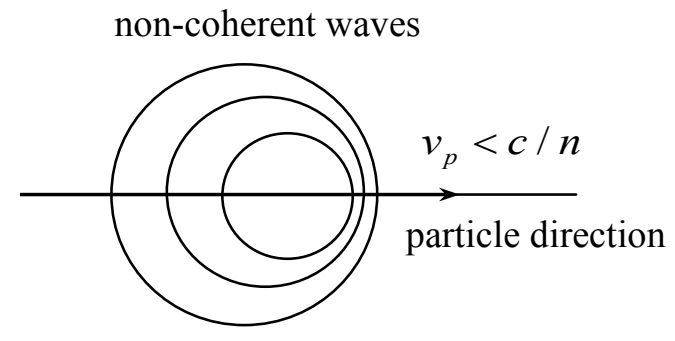

Figure 3.1.1.b Distribution of non-coherent waves in case of an unfulfilled threshold condition.

The characteristic angle $\theta_{c}$ depends on the particle velocity, $v_{p}$, and the properties of the medium: $\cos \left(\theta_{c}\right)=1 / \beta n$, where $n$ is the index of refraction and $\beta=v_{p} / c$ is the relative velocity of the relativistic particle. Out of this dependence, the threshold 
condition for a particle to initiate Cherenkov radiation with non-zero angle (i.e., $\cos \left(\theta_{c}\right)<1$ ) would be: $\beta>1 / n$ or $v_{p}>c / n$. The excited atoms in the medium release the waves with the coherent wavefront in the shape of a cone in the case of the satisfied threshold condition (Fig. 3.1.1.a), and non-coherent waves when the threshold condition is not met (Fig. 3.1.1.b).

It is known that the index of refraction $n$ is a function of temperature and wavelength and so, the emission angle depends on the wavelength $\lambda$ of the Cherenkov radiation. In general, $n$ has a tendency to decrease for higher values of the wavelength. The variation $d n / d \lambda$, referred to as dispersion, becomes the largest in the ultraviolet region. The variation with temperature is generally small enough to be neglected. Typical Cherenkov radiation appears as a continuous spectrum between the ultraviolet and near infrared regions. The number of emitted photons per unit of length along the trajectory and per unit of wavelength is proportional to $1 / \lambda^{2}[66]$ :

$$
\frac{d^{2} N}{d x d \lambda}=\frac{2 \pi \alpha Z^{2}}{\lambda^{2}}\left(1-\frac{1}{\beta^{2} n^{2}}\right)
$$

where $\alpha=e^{2} / \hbar c=1 / 137$ and the incident particle has a charge Ze. It leads to a higher photon yield for shorter wavelength, which means that in the visible electromagnetic spectrum, the radiated blue light dominates over the other colors. In fact, most Cherenkov radiation is actually in the ultraviolet spectrum. In the visible spectrum, the Cherenkov intensity peaks at a blue color. A negligible amount of photons are found in the infrared region. 
The importance of the Cherenkov effect for particle identification lies in the dependence of photon yield (Eq. 3.1.1) and characteristic angle on the particle velocity. By measuring the radiation angle one can uniquely identify the velocity of the incident particle. Independent measurement of the momentum of the traversing particle then allows estimation of the particle's mass $m=p / \beta \gamma$. The Cherenkov radiation however has a problem. It produces a modest photon output in comparison to ionization or excitation based radiation. This means that all possible efforts directed to increase the photon yield are essential for successful particle identification. In general, a Cherenkov detector consists of a radiator with a refractive index $n$ and a photon detection system. The two major types of this detector are differential or focusing Cherenkov detectors and threshold Cherenkov detectors. One of the most advanced types of focusing Cherenkov detectors is the Ring Imaging Cherenkov Detector (RICH) [67]. With the help of an optical system, it focuses the emitted cone of the Cherenkov radiation onto a position sensitive photon detector. After precise measurement of the photon position, the RICH detects a ring, the diameter of which is proportional to the velocity of the particle. The requirement of high spatial resolution for these detectors is essential. Threshold Cherenkov detectors can distinguish common particles with momentum up to $30 \mathrm{GeV} / \mathrm{c}$. These counters utilize the Cherenkov threshold condition $(\beta>1 / n)$ to discriminate the particles. Only particles with velocity above the threshold will create Cherenkov light in the radiator medium. The rest will pass undetected. Since the number of emitted photons depends on the particle velocity (see Equation (3.1.1)), there will be higher photon yield for more energetic particles. Threshold Cherenkov detectors are most useful in 
monochromatic beams where particle differentiation by mass is required. The collection of the photons in such detectors is usually done with PMTs.

In our experiment, E01-011, we used three threshold Cherenkov detectors with silica aerogel radiator. The detailed description of them will be given later in this chapter.

\subsection{Experimental requirements and expectations}

Protons $(p)$, kaons $\left(K^{+}\right)$, and pions $\left(\pi^{+}\right)$pass through the HKS dipole with the same central momentum $p_{0}=1.2 \mathrm{GeV} / \mathrm{c}$ with a $\pm 12.5 \%$ acceptance spread. In this momentum range it is sufficient to utilize a threshold Cherenkov detector for particle discrimination. There were two types of threshold Cherenkov detectors used in the experiment: aerogel Cherenkov detectors (AC) and water Cherenkov detectors (WC). The purpose of AC detectors was the suppression of pions, while the WC detectors were employed to separate protons from kaons. The Cherenkov radiation was detected by photomultiplier tubes. The output of the PMTs is a convolution of the yield of Cherenkov radiation and the response of the collection system. Employing Equation (3.1.1) we can present the number of the detected photoelectrons in the PMTs per particle path length as [66]

$$
\frac{d(\text { n.p.e. })}{d x}=2 \pi \alpha Z^{2} \int\left(1-\frac{1}{\beta^{2} n^{2}}\right) \frac{\varepsilon_{c}(\lambda) R(\lambda)}{\lambda^{2}} d \lambda
$$

where $\varepsilon_{c}(\lambda)$ is the photon collection efficiency and $R(\lambda)$ is the PMT response function. After integration over wavelength and radiator length the number of detected photoelectrons can be expressed as 


$$
\text { n.p.e. }=N_{0} \sin ^{2}(\theta)=N_{0}\left(1-\frac{1}{\beta^{2} n^{2}}\right)=N_{0}\left(1-\frac{m^{2}+p^{2}}{p^{2} n^{2}}\right) \text {. }
$$

The efficiencies and the length of the radiator are absorbed by a constant $N_{0}$.

A plot of n.p.e. as a function of particle momentum is shown in Fig. 3.2.1 for arbitrary $N_{0}=100$. The blue color designates the pions, red the kaons, and green the protons. The plane with the dashed borders represents the particles' central momentum $p=p_{0}=1.2 \mathrm{GeV} / \mathrm{c}$. The $12.5 \%$ momentum acceptance of the dipole HKS magnet is shown on the graph by the grey shaded area. The figure qualitatively shows that for refractive index $n=1.33$, which is equivalent to water, all three particles with central momentum $p_{0}$ produce Cherenkov light. In comparison with protons the yield for pions and kaons is much higher. In the case of the lower index of refraction, $n=1.05$, the only particles creating Cherenkov radiation are pions. From the left figure one can see that for successful discrimination of the pions one has to use a radiator with refractive index of approximately $n<1.10$.

By rewriting the threshold condition $\beta>1 / n$ in the following way

$$
p_{t h}=m / \sqrt{n^{2}-1},(c=1)
$$

we can calculate the minimum momentum required for a particle to create light in the detector's radiator. The threshold momenta (3.2.3) calculated for the particles of interest for two types of Cherenkov radiator material are shown in the Table 3.2.1. A radiator with a refractive index $n=1.05$ corresponds to aerogel material, while a radiator with $n=1.33$ represents water. 


\section{Color code:}

blue - pions, red - kaons, green - protons

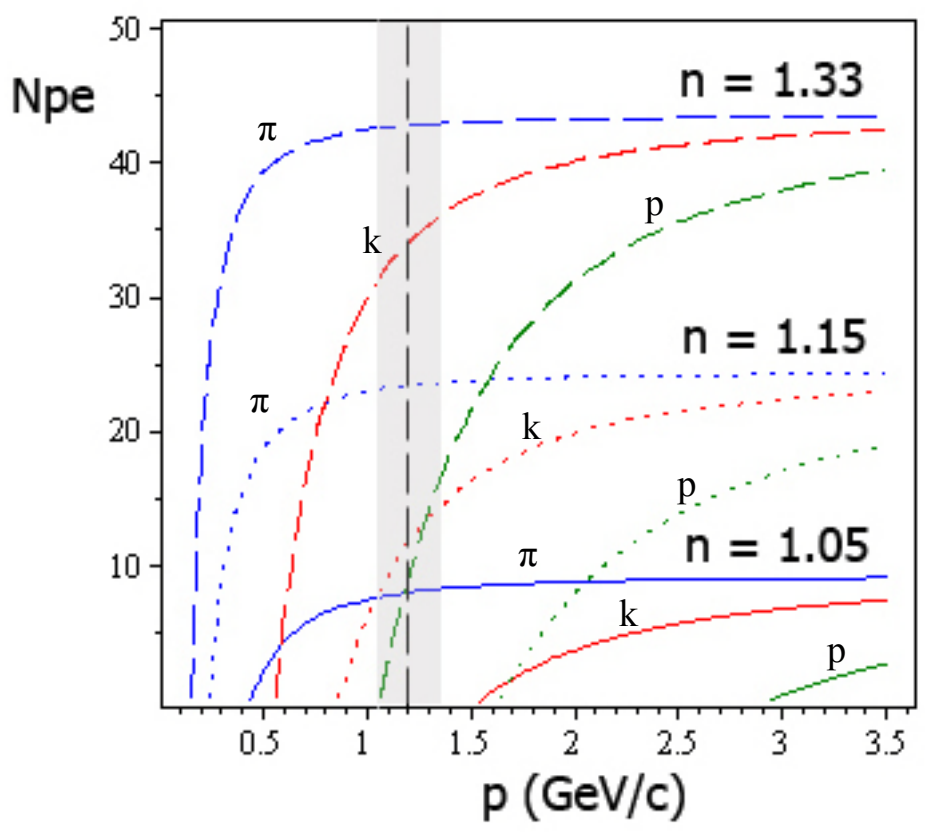

Figure 3.2.1 Cherenkov detector n.p.e. as a function of particle momentum $(p)$ for three values of refractive index of radiator $(n)$. The dashed line represents the central momentum of the HKS spectrometer.

Table 3.2.1 Threshold momentum $p_{\text {th }}$ of particles of interest for select radiator refractive indexes.

\begin{tabular}{|c|c|c|c|}
\hline Particle type - & Pion $\left(m=0.1396 \mathrm{GeV} / \mathrm{c}^{2}\right)$ & Kaon $\left(m=0.4937 \mathrm{GeV} / \mathrm{c}^{2}\right)$ & Proton $\left(m=0.9383 \mathrm{GeV} / \mathrm{c}^{2}\right)$ \\
\hline Rad. ref. index $n=1.05$ & $p_{t h}=0.4360 \mathrm{GeV} / \mathrm{c}$ & $p_{t h}=1.54200 \mathrm{GeV} / \mathrm{c}$ & $p_{t h}=2.9307 \mathrm{GeV} / \mathrm{c}$ \\
\hline Rad. ref. index $n=1.33$ & $p_{t h}=0.1592 \mathrm{GeV} / \mathrm{c}$ & $p_{t h}=0.56300 \mathrm{GeV} / \mathrm{c}$ & $p_{t h}=1.0700 \mathrm{GeV} / \mathrm{c}$ \\
\hline
\end{tabular}

Taking into account the HKS central momentum $p_{0}=1.2 \mathrm{GeV} / \mathrm{c}$, we can see from the table that the aerogel material with $n=1.05$ is perfect for $K^{+} / \pi$ separation, while water $(n=1.33)$ is suitable for a $\operatorname{good} p / K^{+}$discrimination. 


\subsection{Original design and detector characteristics}

As we mentioned in Chapter 2, there are three aerogel Cherenkov counters mounted on the HKS detector rack. The detectors suppressed pions from triggering the HKS electronics by a factor of $10^{-4}$. The schematic design of the aerogel Cherenkov detectors is shown in Fig. 3.3.1. All three AC counters are constructively identically, with a geometrical size of $161 \times 46 \times 20\left(\mathrm{~cm}^{3}\right)$. The counters are organized in three layers positioned by the widest side perpendicular to the direction of incident hadrons ( $z$-axis). The layers are shifted with respect to each other in the particle's dispersive direction (transverse $x$-axis) by approximately $75 \mathrm{~mm}$. Each AC counter consists of the following key elements: 1) container, 2) Cherenkov light radiator, 3) diffusion box, 4) reflector, 5) PMTs.

The counter container was made of aluminum honeycomb panels connected together in a rectangular shape covering four sides of the detector. The widest sides of AC counter, the ones, which are exposed to the incident particles and lie in the $x y$ plane, were covered with $5 \mathrm{~mm}$ thick foam board. The porous foam board was chosen as a light, low-Z material to keep contributions from secondary delta electrons as small as possible. The container was covered one more time by the same foam board material to prevent any possible light leak. The attachment of the cardboard to the frame was done with black vinyl tape. The interior space of the container served as a diffusion box. It was partitioned into seven equal parts, with a size of $23 \times 46 \times 20 \mathrm{~cm}^{3}$. Each part, called a segment, represents an optically separate diffusion box. In comparison with the standard Hall C aerogel Cherenkov detectors, where the internal space has no splitting, the partitioning 
reduces the particle rate inside of a single diffusion box. Assuming a homogeneous distribution of particles over the counter, each segment, on average, receives $1 / 7$ of the total hadron rate. According to Monte-Carlo simulations [68] the segmented geometry of the counter reduced the efficiency by a factor of $1 / 1.2$ because of the increase in the passive area. The separation between segments was achieved by paper layers. The paper was chosen to be relatively thick $(0.5 \mathrm{~mm})$ in order to hold the radiator material in place. For the reasons described earlier, the choice of the radiator was silica aerogel with a refractive index $n=1.05$. Each diffusion box contained a 5 -cm-thick aerogel radiator with an effective area of $23 \times 46 \mathrm{~cm}^{2}$. Since aerogel is a very fragile material, the manufacturing companies frequently produce it in the shape of small rectangular blocks. In our detector we used $11.5 \times 11.5 \times 1.0 \mathrm{~cm}^{3}$ aerogel tiles (SP50) produced by Matsushita Electric Works. 

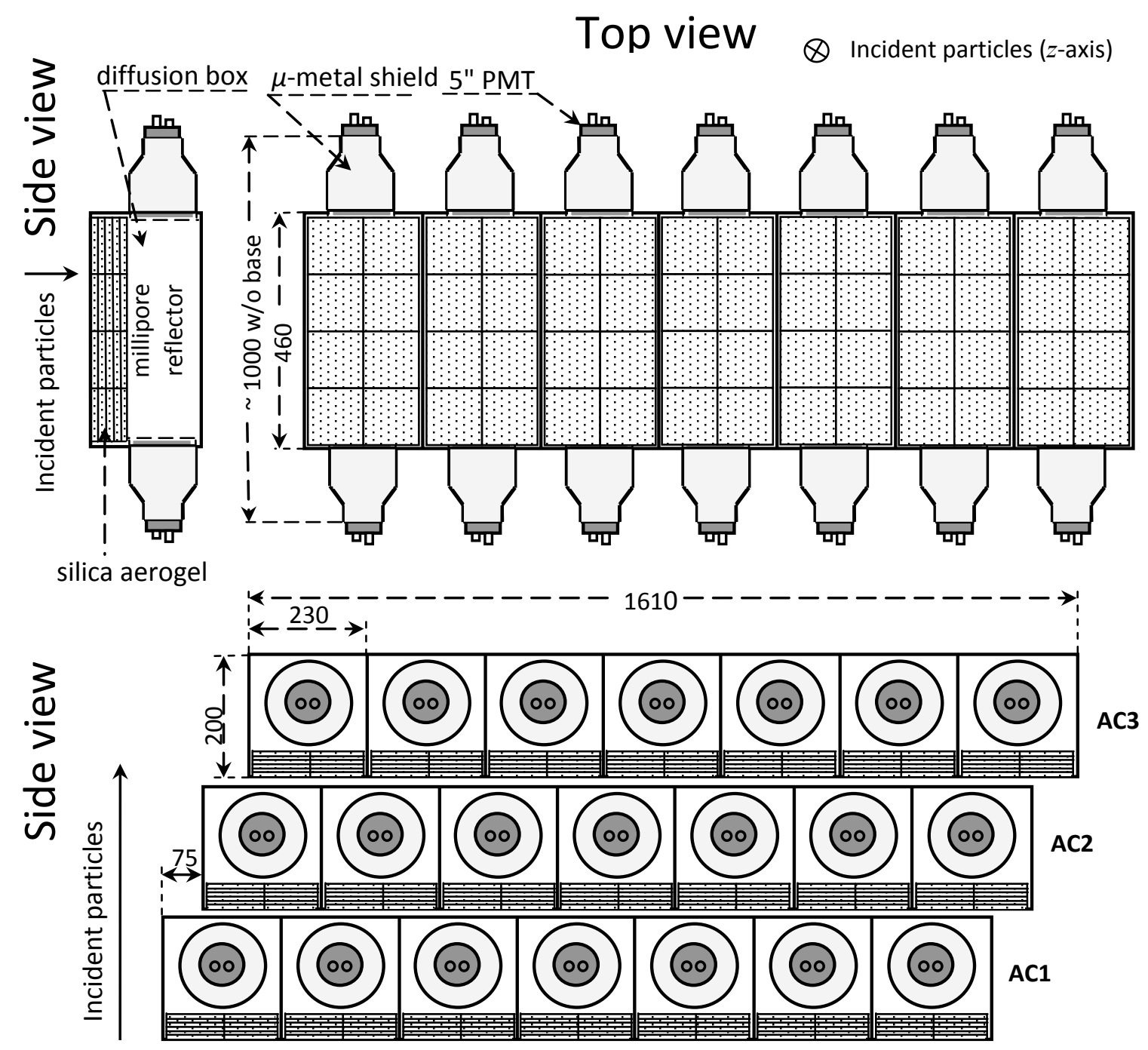

Figure 3.3.1 Schematics of the three aerogel Cherenkov counters (AC1, AC2, AC3). All dimensions are given in $\mathrm{mm}$.

Because this aerogel material is hydrophobic, there are no problems associated with water absorption. Silicon-based aerogel, because of its porous structure with more than $99 \%$ empty space, is almost transparent. Aerogel transmittance spectrum for wavelengths from 220 to $800 \mathrm{~nm}$, measured by [69], is shown in Fig. 3.3.2. The basic properties of aerogel, available on the manufacturer web site, are collected in Table 3.3.1. 
The geometrical properties of the AC counters and main characteristics of the PMTs are shown in the tables 3.3.2 and 3.3.3.

Table 3.3.1 Silica aerogel basic characteristics.

\begin{tabular}{|c|c|c|c|c|c|}
\hline \multirow{2}{*}{$\begin{array}{l}\text { Aerogel } \\
\text { model }\end{array}$} & \multirow{2}{*}{$\begin{array}{c}\text { Tile size } \\
\left(\mathrm{mm}^{3}\right)\end{array}$} & \multirow{2}{*}{$\begin{array}{c}\text { Refractive } \\
\text { index }\end{array}$} & \multirow{2}{*}{$\begin{array}{c}\text { Interaction } \\
\text { depth }\left(\mathrm{g} / \mathrm{cm}^{2}\right)\end{array}$} & \multicolumn{2}{|c|}{ Transmittance } \\
\hline & & & & $\lambda=400 \mathrm{~nm}$ & $\lambda=550 \mathrm{~nm}$ \\
\hline $\begin{array}{l}\text { SP50 } \\
\text { hydrophobic }\end{array}$ & $\begin{array}{c}113 \times 113 \\
\text { (thickness } 10 \text { ) }\end{array}$ & $n=1.05$ & 0.19 & $>62 \%$ & $>88 \%$ \\
\hline
\end{tabular}

Table 3.3.2 Geometrical properties of aerogel Cherenkov (AC) detectors.

\begin{tabular}{cccccccc}
\hline Detector & $\begin{array}{c}\text { Box size } \\
\left(\mathrm{cm}^{3}\right)\end{array}$ & $\begin{array}{c}\text { Number of } \\
\text { segments }\end{array}$ & $\begin{array}{c}\text { Segment } \\
\text { size } \\
\left(\mathrm{cm}^{3}\right)\end{array}$ & $\begin{array}{c}\text { Radiator size } \\
\text { per segment } \\
\left(\mathrm{cm}^{3}\right)\end{array}$ & $\begin{array}{c}\text { Number of } \\
\text { rad. tiles per } \\
\text { segment }\end{array}$ & $\begin{array}{c}\text { Rad. tile } \\
\text { geometry } \\
(\text { tile })\end{array}$ & $\begin{array}{c}\text { Rad. tile size } \\
\left(\mathrm{cm}^{3}\right)\end{array}$ \\
\hline $\mathrm{AC} 1$ & & & & & & & \\
$\mathrm{AC} 2$ & $161 \times 46 \times 20$ & 7 & $23 \times 46 \times 20$ & $23 \times 46 \times 5$ & 40 & $2 \times 4 \times 5$ & $11.5 \times 11.5 \times 1$. \\
$\mathrm{AC} 3$ & & & & & & & \\
\hline
\end{tabular}

Table 3.3.3 General characteristics of PMTs used in AC detectors.

\begin{tabular}{cclcccr}
\hline $\begin{array}{c}\text { Detector } \begin{array}{c}\text { \# of } \\
\text { PMT }\end{array} \\
\text { PMT Model }\end{array}$ & $\begin{array}{c}\text { PMT size } \\
\text { (diameter } \times \text { length with HV base ) }\end{array}$ & Gain & $\begin{array}{c}\text { Spectral } \\
\text { response }(\mathrm{nm})\end{array}$ & HV Base \\
\hline $\mathrm{AC} 1$ & 14 & Hamamatsu R1250 & $5 \square \times 276 \mathrm{~mm}$ & $\approx 10^{7}$ & $300-650$ & C4840 \\
\hline $\mathrm{AC} 2$ & 14 & Photonis XP4572B/D1 & $5 \square \times 276 \mathrm{~mm}$ & $\approx 10^{7}$ & $270-650$ & VD305 \\
\hline $\mathrm{AC} 3$ & 14 & Photonis XP4572B/D1 & $5 \square \times 276 \mathrm{~mm}$ & $\approx 10^{7}$ & $270-650$ & VD305 \\
\hline
\end{tabular}

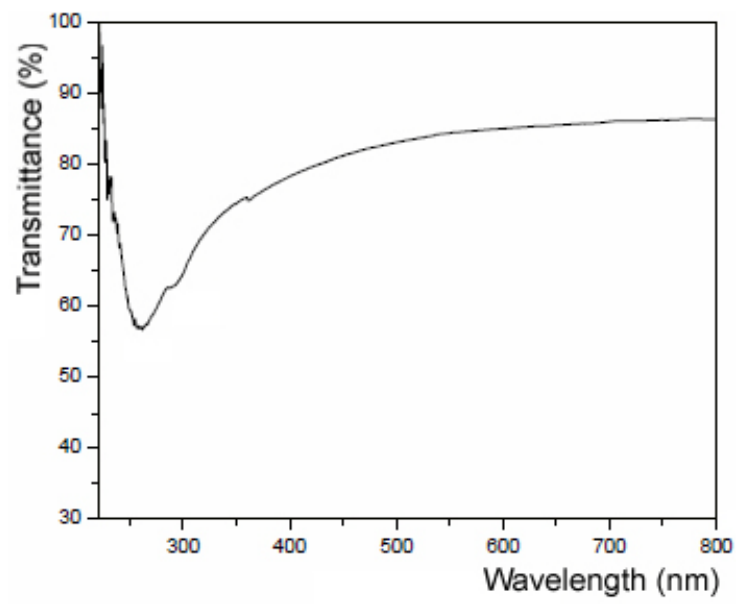

Figure 3.3.2 Dependence of SP-50 aerogel transmittance on the wavelength [69]. 
The aerogel blocks inside of each segment were combined together to form five layers with $2 \times 4$ (tiles) area facing the beam direction (Fig. 3.3.1). The layers were positioned to be held on one side by the cardboard front cover and on the other side by nylon strings, crisscrossed along the segment. The strings were $1 \mathrm{~mm}$ in diameter in order to withstand the weight of all 40 aerogel tiles and at the same time to minimize the unnecessary interaction of the strings with the incident particles. Each diffusion box contains two PMTs located on the top and bottom of the segment (Fig. 3.3.3). We used two types of the photomultiplier tubes: Photonis XP4572B/D1 and Hamamatsu R1250. Both types have almost the same geometrical properties (5" collection diameter and $27.6 \mathrm{~cm}$ in length). They were all operated in positive HV mode (cathode ground scheme). To increase the signal strength, an additional amplifier, designed at Jefferson Lab [70], was built into the PMT base, which also houses the high voltage divider. The operational voltage on PMTs during the experiment was around $1800 \mathrm{~V}$. According to the documentation from the PMT manufacturers such a voltage results in up to a $10^{7}$ gain. As the Table 3.3.3 shows we used a total of 14 Hamamatsu PMTs on AC1 and 28 Photonis PMTs on AC2 and AC3. Both tube types have approximately the same spectral response, ranging from 300 (or 270) $-650 \mathrm{~nm}$. The maximum response of the tubes is achieved for incident photons with $420 \mathrm{~nm}$ wavelength, which corresponds to violet-blue color on the visible light spectrum. Because the Cherenkov radiation has high intensity in this wavelength region, these PMTs well suited for application in our AC detectors.

The incident beam of hadrons traversing the aerogel radiator creates Cherenkov radiation directed inside of the segment. Because the PMTs are mounted far on the ends of the segment, the diffusion box must have a high reflectivity to successfully deliver 
photons to the tube. That is achieved with white Millipore paper GSWP00010 used as a diffuse reflector. Made of pure cellulose fibers, Millipore has $95 \%$ reflectivity in the 350 - $450 \mathrm{~nm}$ region and it is commonly used in industry as a filter membrane [71]. The Millipore paper was attached with double-sided tape to all internal walls in each segment, except the aerogel surface and the round holes for the PMTs (Fig. 3.3.3). One concern was to keep the Millipore reflectivity as close to its manufactured value as possible. Thus, assembly of the detector took place in a clean room, with personnel wearing vinyl gloves.

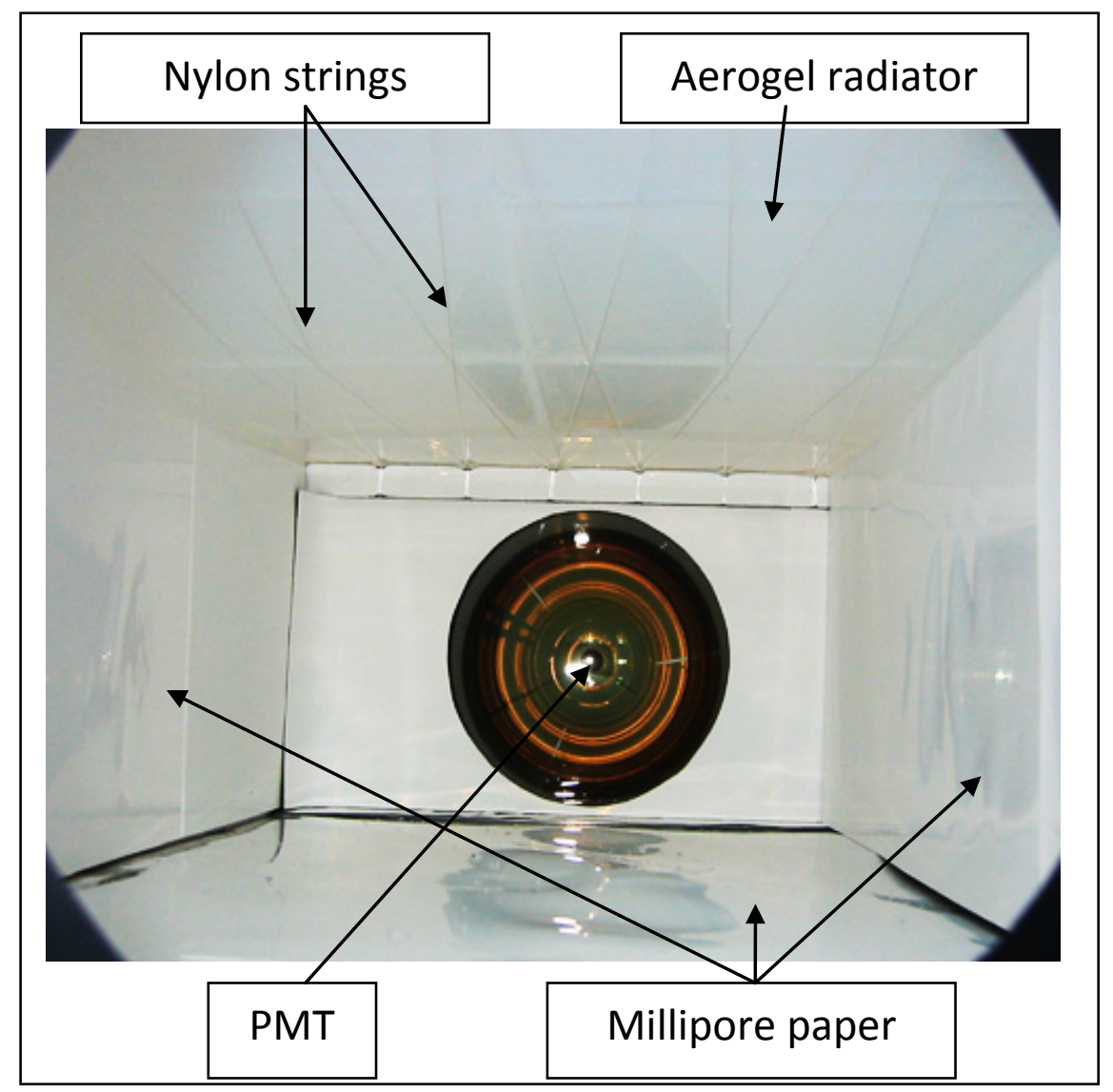

Figure 3.3.3 Interior of the diffusion box (view from the PMT hole) [56]. 


\subsection{Performance}

\subsubsection{KEK and JLab test results}

Within the E01-011 (HKS) collaboration, Florida International University was responsible for the development and construction of the aerogel Cherenkov detectors. The author, under supervision of his advisor and a great deal of help from his colleagues, was directly involved in the on-site detector fabrication and testing. Prior to the final detector assembly in Jefferson Lab's clean room (EEL building), a one-segment counter prototype was built by a graduate student at FIU and tested in the particle beam at KEK (Japan). A Monte-Carlo simulation of the prototype counter predicted 19.76 photoelectrons for particles at $1.2 \mathrm{GeV} / \mathrm{c}$. A cosmic ray test with high energy, $\sim 4 \mathrm{GeV}$, muons averaged 25 photoelectrons [68]. The particle beam test was conducted at the 12 $\mathrm{GeV}$ proton synchrotron facility at KEK. $1.2 \mathrm{GeV} / \mathrm{c}$ pions resulted in a measured number of 15.4 photoelectrons, which showed that the real detector efficiency is less than estimated by Monte-Carlo [72].

After we fabricated all three aerogel Cherenkov counters in the Jefferson Lab clean room (EEL building), quality tests were initiated. A locally arranged data acquisition system was triggered on one of the PMTs in order to measure the single

photoelectron peak on the other tube. The cosmic rays were triggered by two scintillator bars positioned in the form of a cross and placed on top of the AC box (Fig. 3.4.1). On average 20 photoelectrons in $\mathrm{AC} 1$ and $\mathrm{AC} 3$, and 23 in $\mathrm{AC} 2$ were observed, Table 3.4.1. 


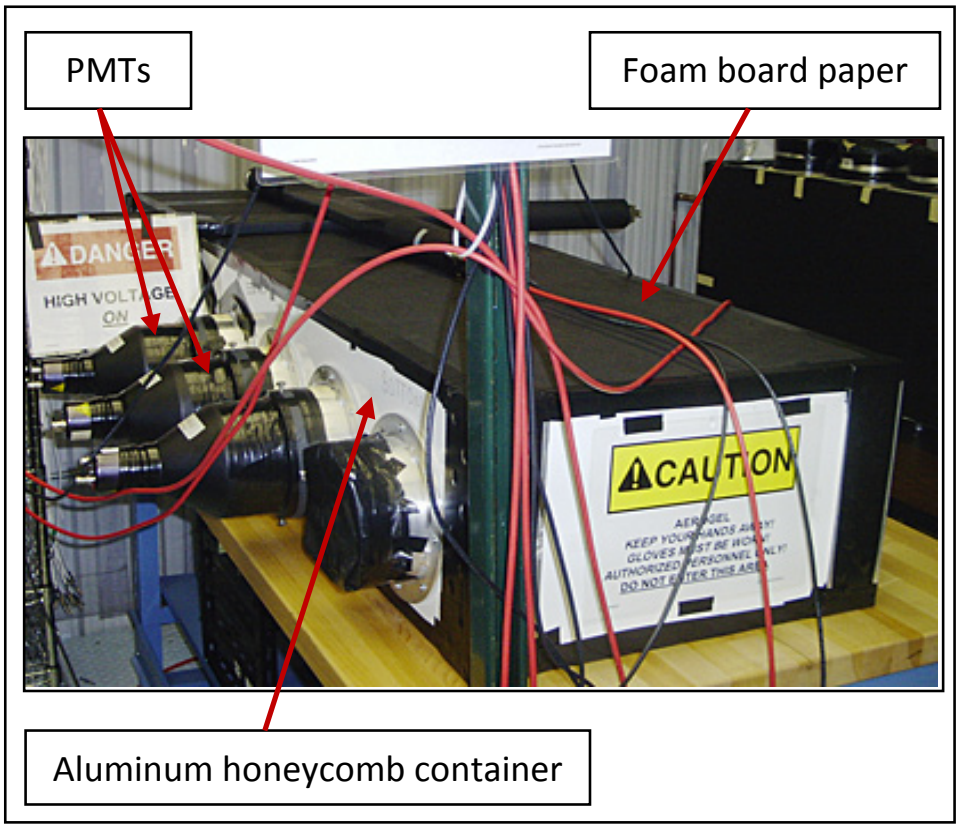

Figure 3.4.1 AC counter under test in EEL (JLab).

In the following we define a gain as ADC channels per photoelectron:

$$
\text { Gain }=1 \text { p.e. }- \text { Pedestal } \text {. }
$$

Note, that this is related to the PMT gain, $g_{\mathrm{PMT}}$, amplifier gain, $g_{\mathrm{AMP}}$, and ADC sensitivity of $50 \mathrm{fC} /$ channel by Gain $=g_{\mathrm{PMT}} \cdot g_{\mathrm{AMP}} \cdot e / 50 \mathrm{fC}$. The 1p.e. in Equation 3.4 .1 means the single photoelectron peak. The total number of photoelectrons, n.p.e. is therefore estimated as

$$
\text { n.p.e. }=\frac{\text { ADCsignal }- \text { Pedestal }}{\text { Gain }} \text {. }
$$


Table 3.4.1 Results of AC counters performance tested in EEL clean room at JLab.

\begin{tabular}{|c|c|c|c|c|c|c|c|c|}
\hline & Segment & PMT & Label & PMT Model & PMT HV (V) & PMT Gain & n.p.e. & Summed n.p.e. \\
\hline \multirow{14}{*}{ } & \multirow{2}{*}{1} & $1 \mathrm{~T}$ & RAXX09 & H. R1250 & 1890 & 139.7 & 8.5 & \multirow{2}{*}{19.4} \\
\hline & & 1B & RAXX08 & H. R1250 & 1890 & 130.6 & 6.7 & \\
\hline & \multirow[b]{2}{*}{2} & $2 \mathrm{~T}$ & RAXX07 & H. R1250 & 1910 & 146.8 & 11.1 & \multirow[b]{2}{*}{20.1} \\
\hline & & $2 \mathrm{~B}$ & RA2122 & H. R1250 & 1710 & 135.3 & 7.1 & \\
\hline & \multirow{2}{*}{3} & $3 \mathrm{~T}$ & RA2209 & H. R1250 & 2100 & 146.8 & 7.4 & \multirow{2}{*}{18.7} \\
\hline & & 3B & RA2123 & H. R1250 & 1950 & 150.7 & 7.2 & \\
\hline & \multirow{2}{*}{4} & $4 \mathrm{~T}$ & RA2140 & H. R1250 & 1830 & 135.8 & 8.4 & \multirow{2}{*}{20.5} \\
\hline & & $4 \mathrm{~B}$ & RAXX04 & H. R1250 & 1950 & 139.1 & 8.0 & \\
\hline & \multirow{2}{*}{5} & $5 \mathrm{~T}$ & RAXX03 & H. R1250 & 1710 & 158.9 & 7.9 & \multirow{2}{*}{18.5} \\
\hline & & $5 \mathrm{~B}$ & RA2126 & H. R1250 & 1850 & 169.5 & 7.7 & \\
\hline & \multirow{2}{*}{6} & $6 \mathrm{~T}$ & RA2202 & H. R1250 & 2000 & 160.7 & 11.1 & \multirow{2}{*}{22.7} \\
\hline & & $6 \mathrm{~B}$ & RA2143 & H. R1250 & 1800 & 162.1 & 9.7 & \\
\hline & \multirow{2}{*}{7} & $7 \mathrm{~T}$ & RAXX02 & H. R1250 & 1800 & 191.6 & 10.0 & \multirow{2}{*}{20.4} \\
\hline & & $7 \mathrm{~B}$ & RAXX01 & H. R1250 & 1950 & 134.9 & 8.2 & \\
\hline \multirow{14}{*}{ 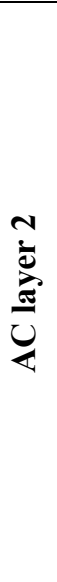 } & \multirow{2}{*}{1} & $1 \mathrm{~T}$ & A12 & Ph. XP4572B/D1 & $1600-1700$ & 24.5 & $10-15$ & \multirow{2}{*}{26.6} \\
\hline & & $1 \mathrm{~B}$ & A11 & Ph. XP4572B/D1 & $1600-1700$ & 30.6 & $10-15$ & \\
\hline & \multirow{2}{*}{2} & $2 \mathrm{~T}$ & B12 & Ph. XP4572B/D1 & $1600-1700$ & 35.3 & $10-15$ & \multirow{2}{*}{23.4} \\
\hline & & $2 \mathrm{~B}$ & B11 & Ph. XP4572B/D1 & $1600-1700$ & 23.9 & $10-15$ & \\
\hline & \multirow{2}{*}{3} & $3 \mathrm{~T}$ & $\mathrm{C} 12$ & Ph. XP4572B/D1 & $1600-1700$ & 26.3 & $10-15$ & \multirow{2}{*}{25.7} \\
\hline & & $3 \mathrm{~B}$ & $\mathrm{C} 11$ & Ph. XP4572B/D1 & $1600-1700$ & 28.0 & $10-15$ & \\
\hline & \multirow{2}{*}{4} & $4 \mathrm{~T}$ & D12 & $\mathrm{Ph} . \mathrm{XP} 4572 \mathrm{~B} / \mathrm{D} 1$ & $1600-1700$ & 21.1 & $10-15$ & 230 \\
\hline & & 4B & D11 & Ph. XP4572B/D1 & $1600-1700$ & 22.8 & $10-15$ & 23.0 \\
\hline & 5 & $5 \mathrm{~T}$ & E12 & $\mathrm{Ph} . \mathrm{XP} 4572 \mathrm{~B} / \mathrm{D} 1$ & $1600-1700$ & 30.4 & $10-15$ & 255 \\
\hline & 5 & $5 \mathrm{~B}$ & E11 & Ph. XP4572B/D1 & $1600-1700$ & 31.0 & $10-15$ & 23.5 \\
\hline & 6 & $6 \mathrm{~T}$ & F12 & $\mathrm{Ph} . \mathrm{XP} 4572 \mathrm{~B} / \mathrm{D} 1$ & $1600-1700$ & 24.1 & $10-15$ & 237 \\
\hline & 0 & $6 \mathrm{~B}$ & F11 & Ph. XP4572B/D1 & $1600-1700$ & 27.4 & $10-15$ & 23.1 \\
\hline & & $7 \mathrm{~T}$ & G12 & Ph. XP4572B/D1 & NA & NA & NA & \\
\hline & t & $7 \mathrm{~B}$ & G11 & Ph. XP4572B/D1 & NA & NA & NA & NA \\
\hline & 1 & $1 \mathrm{~T}$ & 60326 & Ph. XP4572B/D1 & 2000 & 139.7 & 8.5 & 0 \\
\hline & 1 & $1 \mathrm{~B}$ & 60340 & Ph. XP4572B/D1 & 1950 & 130.6 & 6.7 & 19.4 \\
\hline & $?$ & $2 \mathrm{~T}$ & 60323 & Ph. XP4572B/D1 & 1750 & 146.8 & 11.1 & م \\
\hline & 2 & $2 \mathrm{~B}$ & 60192 & Ph. XP4572B/D1 & 2000 & 135.3 & 7.1 & 20.1 \\
\hline & 3 & $3 \mathrm{~T}$ & 60178 & $\mathrm{Ph} . \mathrm{XP} 4572 \mathrm{~B} / \mathrm{D} 1$ & 2250 & 146.8 & 7.4 & 187 \\
\hline$m$ & 3 & 3B & 60322 & Ph. XP4572B/D1 & 1800 & 150.7 & 7.2 & 18.1 \\
\hline હ & 4 & $4 \mathrm{~T}$ & 60327 & Ph. XP4572B/D1 & 1800 & 135.8 & 8.4 & 205 \\
\hline$\underline{G}$ & 4 & 4B & 60328 & Ph. XP4572B/D1 & 1750 & 139.1 & 8.0 & 20.5 \\
\hline 4 & 5 & $5 \mathrm{~T}$ & 60324 & $\mathrm{Ph} . \mathrm{XP} 4572 \mathrm{~B} / \mathrm{D} 1$ & 1950 & 158.9 & 7.9 & 185 \\
\hline & 5 & $5 \mathrm{~B}$ & 60325 & Ph. XP4572B/D1 & 1750 & 169.5 & 7.7 & 18.5 \\
\hline & 6 & $6 \mathrm{~T}$ & 60341 & $\mathrm{Ph} . \mathrm{XP} 4572 \mathrm{~B} / \mathrm{D} 1$ & 2250 & 160.7 & 11.1 & 27 \\
\hline & 0 & $6 \mathrm{~B}$ & 60332 & Ph. XP4572B/D1 & 1900 & 162.1 & 9.7 & 22.1 \\
\hline & 7 & $7 \mathrm{~T}$ & 60339 & $\mathrm{Ph} . \mathrm{XP} 4572 \mathrm{~B} / \mathrm{D} 1$ & 2180 & 191.6 & 10.0 & 204 \\
\hline & t & 7B & 60331 & Ph. XP4572B/D1 & 1950 & 134.9 & 8.2 & 20.4 \\
\hline
\end{tabular}


An example of the single-photoelectron peak and the ADC signal distribution during the test is shown in Fig. 3.4.2.

The self trigger on the PMT allows the single photoelectron peak to be visible with high statistics and resolution (left plot). The right plot corresponds to a cosmic test on high energy muons with the trigger set on the coincidences between the two scintillator bars.
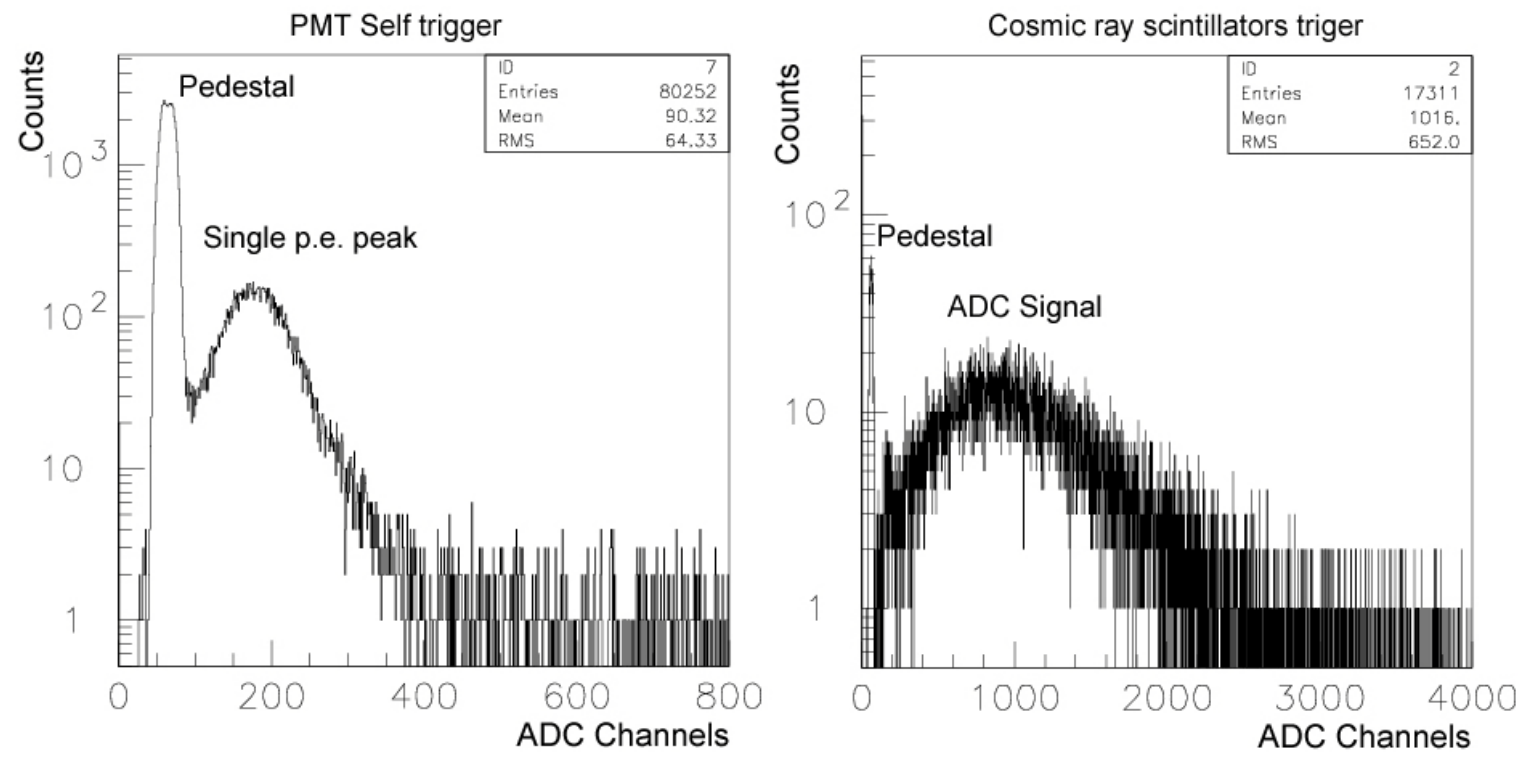

Figure 3.4.2 Example of the single photoelectron (p.e.) peak and cosmic ADC peak during testing at JLab clean room.

\subsubsection{Gain matching}

To equalize the response of the photomultiplier tubes, the applied High Voltage (HV) had to be adjusted individually for each tube to provide an equal gain. The gain match procedure consisted of performing self trigger runs for each PMT at several HV values. 
An example of the gain match for two PMTs in the first segment of the AC1 layer is shown in the Table 3.4.2.

The data points were fitted with exponential, Gain $=A \cdot \exp (B \cdot H V)$ (Fig. 3.4.3). Parameters A and B were extracted for all $7 \times 2 \times 3=42$ PMTs and recorded in the gain tables that were used during the run period. During experiment commissioning, each PMT was adjusted to Gain $=90$. If necessary, this value could be easily varied with the help of the CAEN HV crates installed in the CEBAF counting house.

Table 3.4.2 Gain matching for AC1 segment 1 (1T and 1B tubes).

\begin{tabular}{cccc|cccc}
\hline \multicolumn{3}{c|}{ PMT 1T (AC1) } & \multicolumn{4}{c}{ PMT 1B (AC1) } \\
\hline \multicolumn{3}{c}{ Self Trigger: } & On 1B PMT & \multicolumn{3}{c}{ Self Trigger: } & On 1T PMT \\
\hline HV (V) & Pedestal & 1 p.e. & Gain & HV (V) & Pedestal & 1 p.e. & Gain \\
\hline 1800 & 450.8 & 525.6 & 74.8 & 1800 & 432.3 & 500.3 & 68.0 \\
1850 & 450.7 & 551.6 & 100.9 & 1850 & 432.7 & 528.8 & 96.1 \\
1900 & 453.4 & 591.2 & 137.8 & 1900 & 434.2 & 563.9 & 129.7 \\
1910 & 456.3 & 609.6 & 153.3 & 1920 & 437.9 & 585.3 & 147.4 \\
1950 & 454.7 & 644.6 & 189.9 & 1950 & 435.4 & 612.6 & 177.2 \\
\hline
\end{tabular}
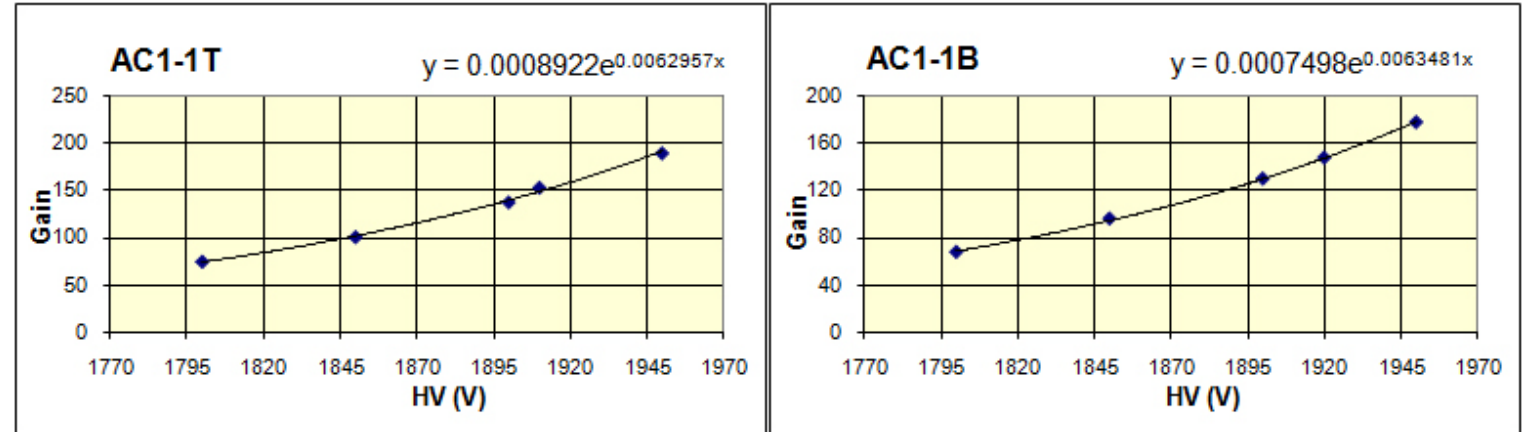

Figure 3.4.3 Gain dependence on applied HV for AC1 segment 1.

Since the AC counters are participating in the hadron trigger, the appropriate threshold had to be applied to the counters' anode signals. A scan was done for all AC segments in each detector layer. Because the threshold had to be set only for a whole 
layer, the values were averaged. Figure 3.4.4 shows the threshold scan for each AC layer. With the knowledge of the gain values the ADC channel cutoff values were converted into a n.p.e. cutoff. The thresholds were set just above the single photoelectron peak, which allowed an effective pion selection for further veto in the hadron trigger. A linear fit was applied to the test data in order to provide an analytical equation for further adjustment of the threshold values.

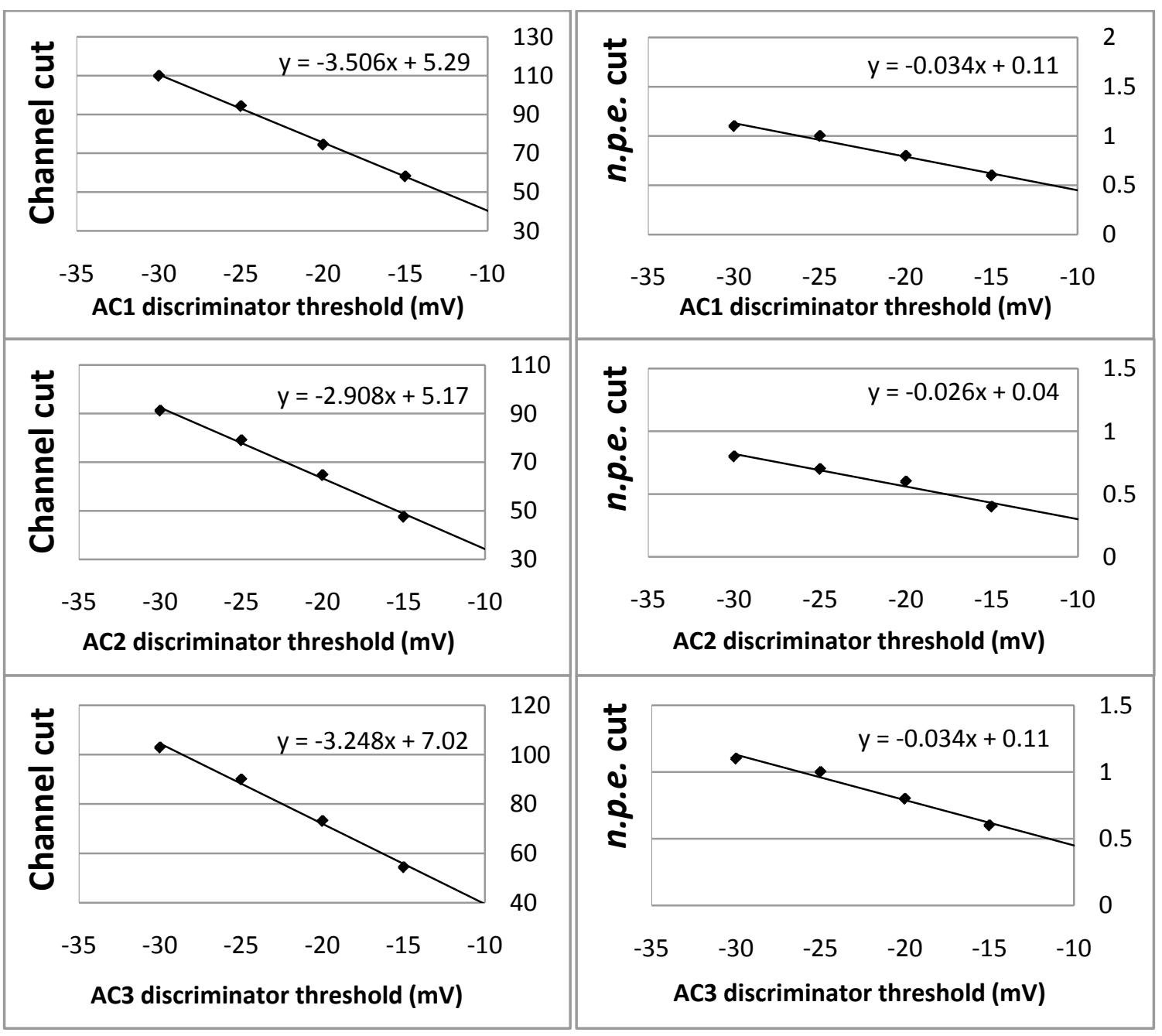

Figure 3.4.4 AC threshold scan. (Cut is shown in Channels and $\mathrm{mV}$ ). 


\subsubsection{Bucking coils}

As we mentioned earlier, the photons created in Cherenkov radiation were detected with the help of photomultiplier tubes. A primitive schematic of a PMT is shown in Fig. 3.4.5. The photons liberate the electrons from the photocathode by the photoelectric effect. These few electrons are not of sufficient number to be reliably detected by electronics. Thus, inside of the PMT they are attracted to positively charged electrodes, called dynodes. Each electron upon interaction with a dynode knocks out several more electrons creating an electron avalanche that moves along the dynodes in the tube. Each dynode is set at a more positive electrical potential than the previous. Such an arrangement allows amplification of the tiny current of electrons - typically by a factor of 1-10 million.

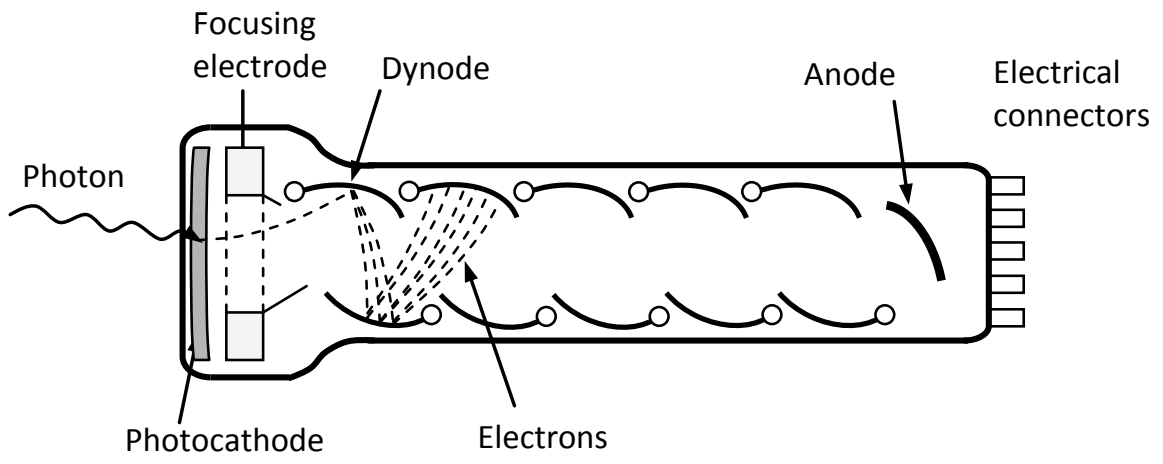

Figure 3.4.5 Photomultiplier tube schematic diagram.

Since the PMTs deal with a free electron current inside the tube, they are sensitive to magnetic fields. To protect the PMT, the manufacturers enclose the tube in a shield made of high permeability soft Nickel-Iron material ( $\mu$-metal). All of the detectors in our experiment used a protective shielding mounted on PMTs. 
During the experiment run period there was a significant magnetic field present in the ENGE and HKS huts. The powerful magnetic spectrometers served as an origin of a fringe magnetic field that extended beyond the confines of the magnets' yokes. In the HKS bunker, a survey showed the presence of fringe fields up to 10 Gauss. At the position of the HKS detector platform the magnetic field was non-uniformly distributed ranging in strength from 3 to 6 Gauss. Out of all detectors, the aerogel Cherenkov with the 5" PMTs suffered the largest impact from this fringe magnetic field. The direction of the magnetic field is shown schematically in Fig. 3.4.6. The shape of the fringe field surrounding the magnet was not a perfect circle. It rather reproduced the shape of the magnet with some possible anomalies on the surface. However, in the vicinity of the HKS detector rack the lines were directed vertically which aligns them with the central axis of the PMT tubes in the detectors. That allows the field lines to directly access the focusing area of the PMT, i.e. the space between photocathode and first dynode (Fig. 3.4.7).

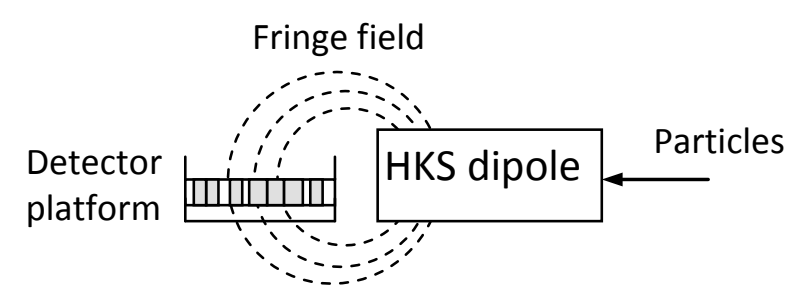

Figure 3.4.6 Schematics of the HKS setup and relative direction of fringe field lines.

Under the influence of the magnetic field, photoelectrons that follow electric field lines at an angle with respect to the magnetic field will move in spiral trajectories because 
of the magnetic Lorentz force. Such parameters as the strength of magnetic field and the geometry of the first PMT section (from photocathode to first dynode) are crucial for optimizing collection efficiency.

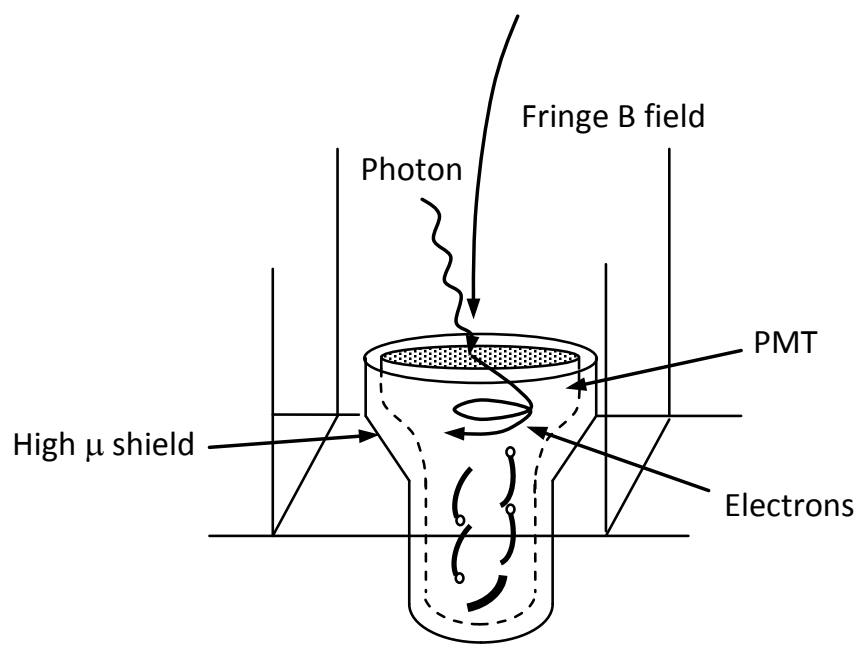

Figure 3.4.7 Electron trajectories within PMT under influence of fringe field.

There was no significant reduction in collection efficiency observed for PMTs with a relatively small effective photocathode area. However, the aerogel Cherenkov PMTs, with a 127-mm-diameter photocathode suffered serious consequences from the fringe field. Figure 3.4.8 shows this effect on the ADC distribution for one of the AC detector PMTs. The test was performed during the experiment commissioning period without beam. These data were obtained by pulsing an LED inside of the segment. With the HKS magnet powered to its operational current the ADC signal dropped by more than a factor of two when compared to the magnet OFF state.

Under this condition, it is almost impossible to conduct proper particle identification. To minimize the field's effect, an iron bunker was built around the detector 
stack. The magnetic field lines were expected to be trapped in the roof and the walls of this bunker and to be shortened by the iron flooring installed in the HKS hut. Figure 3.4.9 shows the metallic strips installed behind detectors. Identical strips were installed from top, left and right sides, resting on the iron framework constructed around the detector rack.

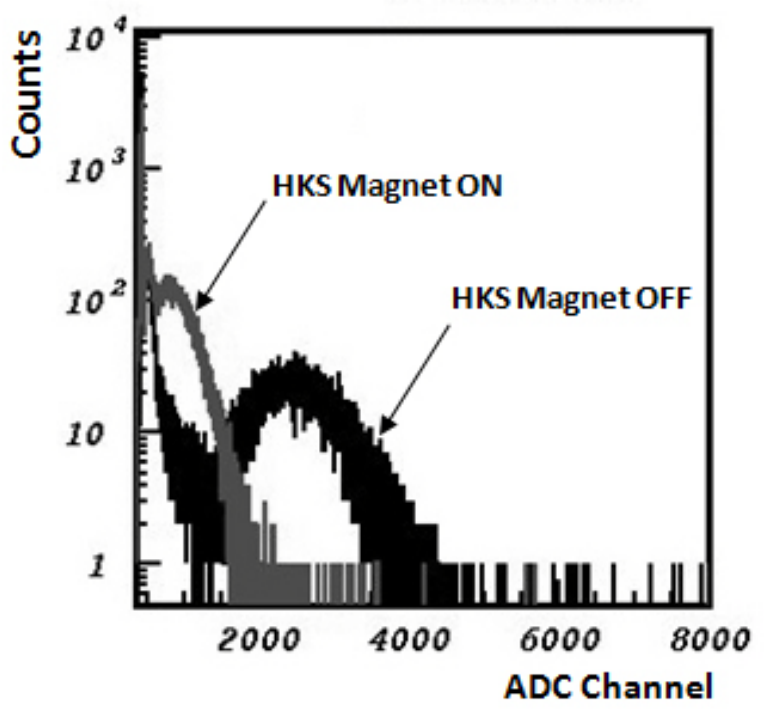

Figure 3.4.8 Effect of the fringe magnetic field on aerogel Cherenkov ADC signal.

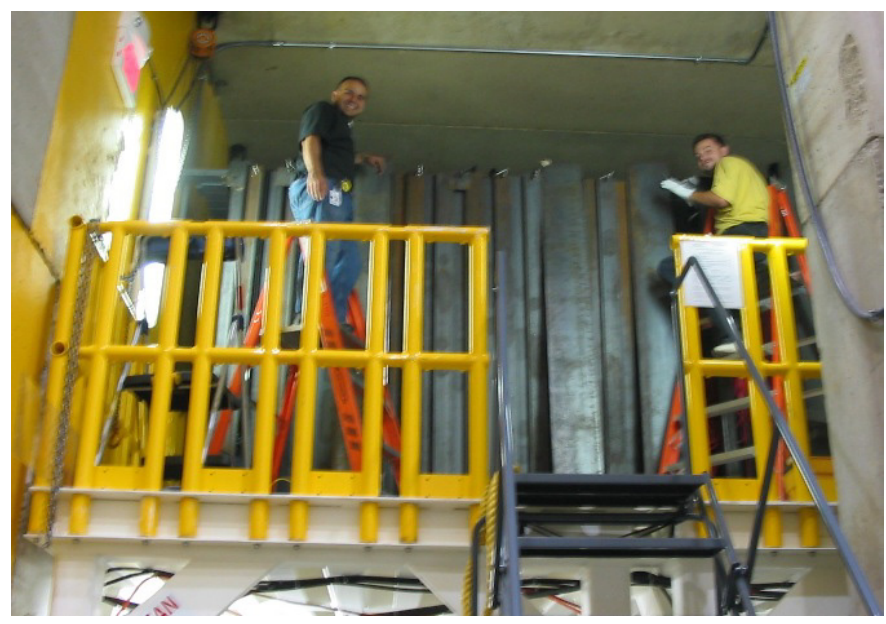

Figure 3.4.9 View of the HKS hut interior [56]. The iron bunker is constructed around the detector crate to minimize the fringe field in the vicinity of the detectors. 
This shield bunker led to a partial reduction of the magnetic field. Measurements with a hall probe showed a more uniform distribution of the field around the detectors with a strength of $\approx 2.5$ Gauss. This reduction, however, unfortunately did not result in a complete recovery of the PMTs' ADC signal.

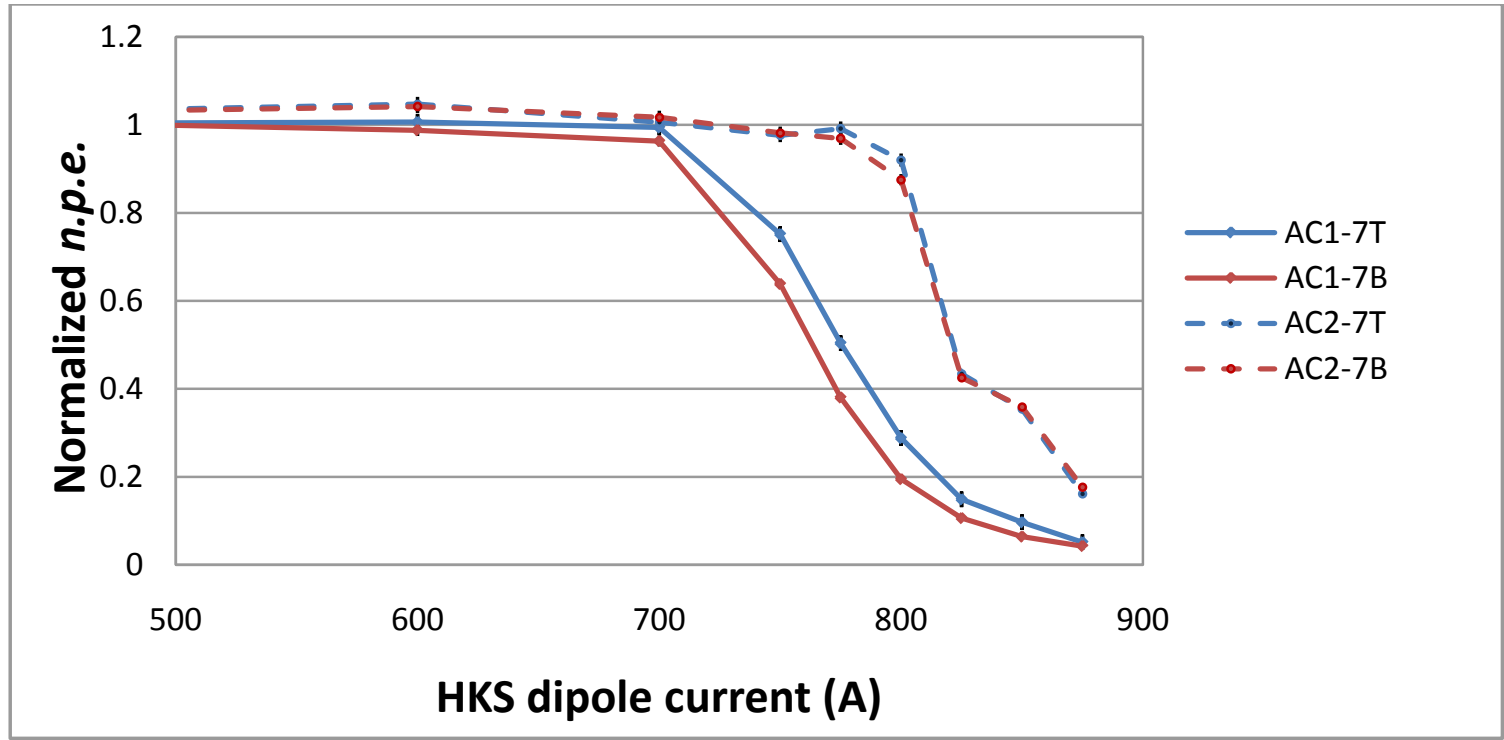

Figure 3.4.10 Reduction of the n.p.e. with increase of the current in the HKS dipole.

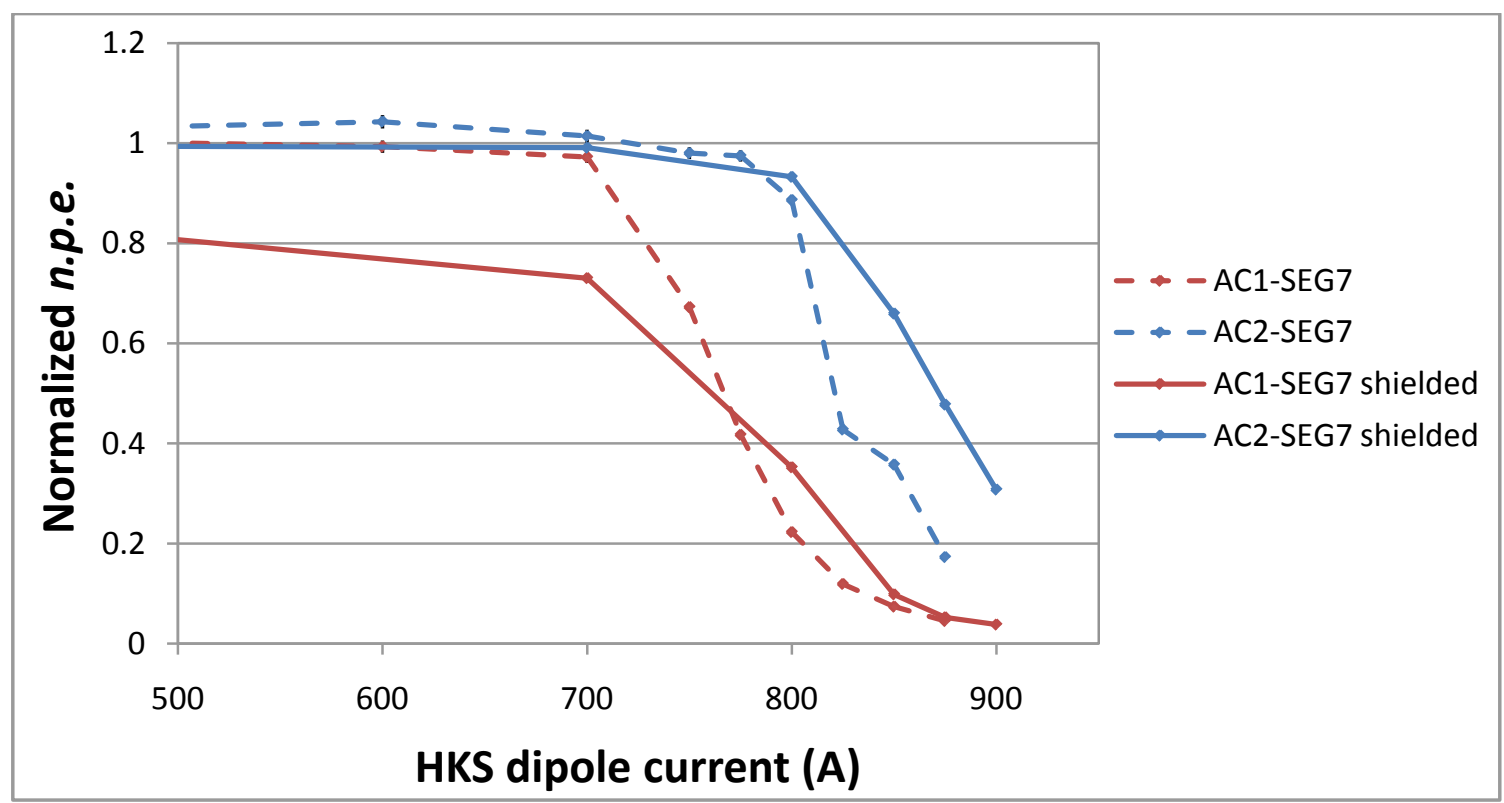

Figure 3.4.11 Improvement of n.p.e. in the presence of the iron bunker. 
Figures 3.4.10 and 3.4.11 show the change of the normalized number of photoelectrons in selected PMTs $(\mathrm{AC} 1-7 \mathrm{~T} \& \mathrm{~B}$ and $\mathrm{AC} 2-7 \mathrm{~T} \& \mathrm{~B})$ as a function of the current in the HKS dipole. As the Fig. 3.4.11 shows, there is only partial recovery of the signal. It is worth mentioning that these measurements were performed at currents well below the magnet's nominal current of $1060 \mathrm{~A}$.

Therefore, another approach to restore the signal in the PMTs was incorporated. The fringe field that penetrated inside of the tube was compensated by an opposite magnetic field locally created by a current carrying coil. This was established by 12 AWG wires coiled around the widest part of the PMT (Fig. 3.4.12). A photograph of the PMTs with these bucking coils is shown in Fig. 3.4.13.

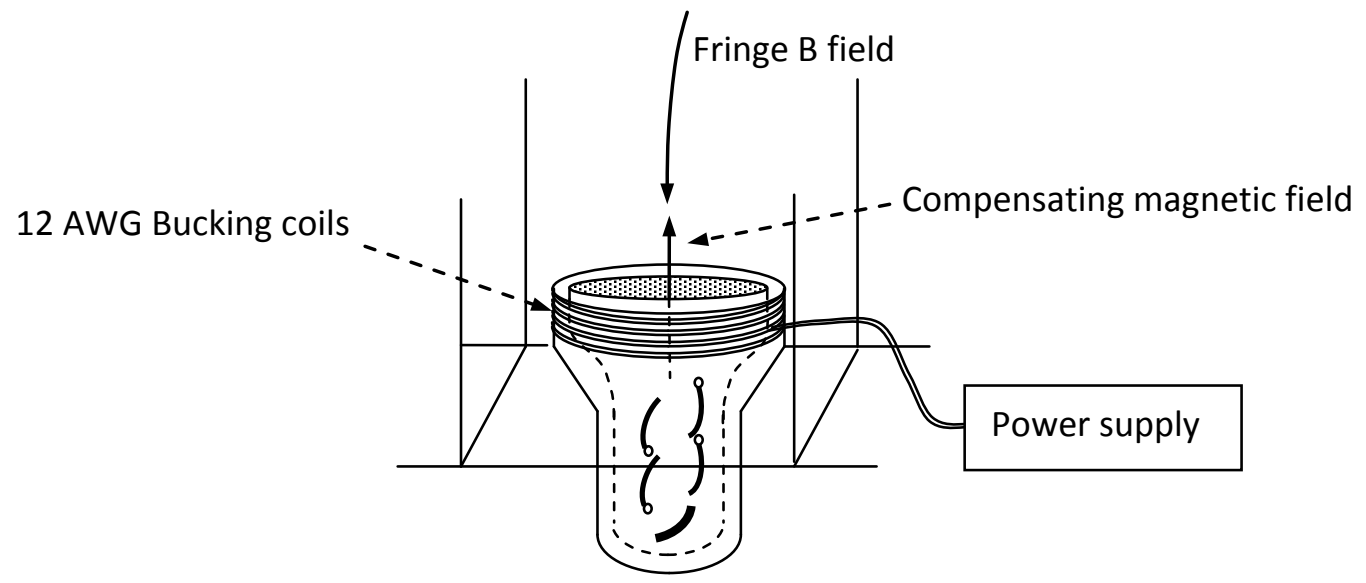

Figure 3.4.12 Bucking coils with compensating local magnetic field.

As this bucking coil system was a last minute response to a larger than anticipated fringe field, only three segments, one in each layer, were tested with an LED. In total three segments were tested: AC1-7, AC2-7 and AC3-7. 


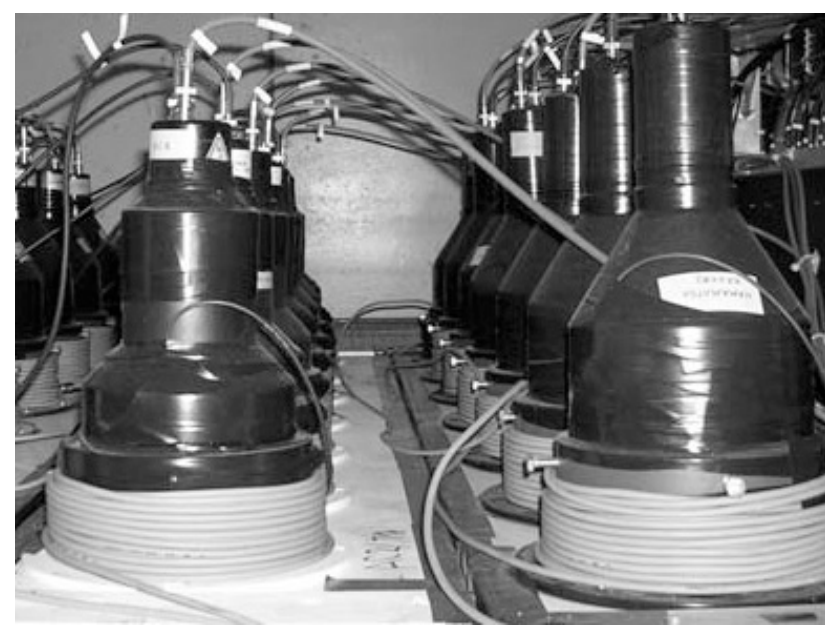

Figure 3.4.13 Photo of AC PMTs with bucking coils installed [56].

Figure 3.4.14 presents the result of the change of normalized n.p.e. with increase of the current in the bucking coils. The first three plots show the performance of the "Top" (T) and "Bottom" (B) PMTs for segments AC1-7, AC2-7, and AC3-7 with the HKS dipole magnet powered at the operational current of 1060 A. The normalization was done with respect to the run with zero currents in the bucking coils and dipole magnet. The last plot shows the summed $\mathrm{T}$ and B signals. During the test runs the AC1-7B PMT had no protective shield installed. Therefore its recovery curve looks different from the rest of the tested PMTs. Based on these plots, it was decided to set the following currents for powering the bucking coils: $I_{A C 1}=3.2 \mathrm{~A} ; I_{A C 2}=2.2 \mathrm{~A} ; I_{A C 3}=2.2 \mathrm{~A}$. There were three power supplies installed in the HKS hut that provided the current to each layer. Each layer used one piece of wire which ran along the PMTs sequentially coiling around each tube. 


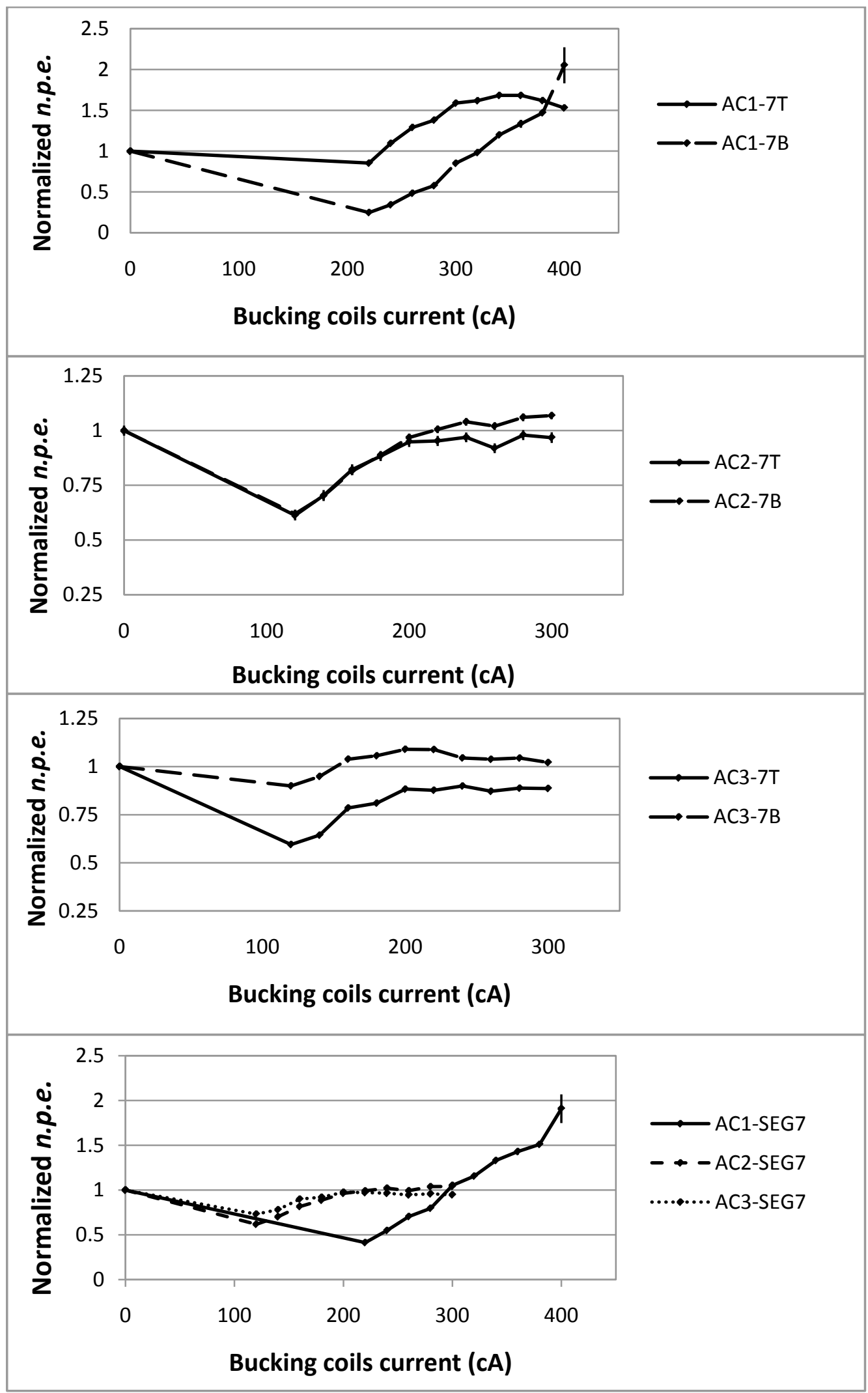

Figure 3.4.14 Bucking coils LED test for segment 7 of AC1, AC2, and AC3. 


\subsubsection{Performance during the experiment}

After the gain match procedure, described in section 3.4.2, the photomultiplier tubes had roughly equal responses. Self-trigger runs were done during commissioning time with beam and the Gain values were recorded in the parameter files for further use in the analyzer routine. During the experiment production period the performance of AC counters was satisfactory, although the n.p.e. yield was lower than was expected.

On average the number of photoelectrons ranged from 6 to 10 in one $\mathrm{AC}$ layer. This yield varied over time (shown in Fig. 3.4.15) as a function of run number. Such knowledge is important for the particle identification analysis, which we will describe in the next chapter. The n.p.e. signal in AC1 decreases with time, while the signals in AC2 and $\mathrm{AC} 3$ show an increase. 


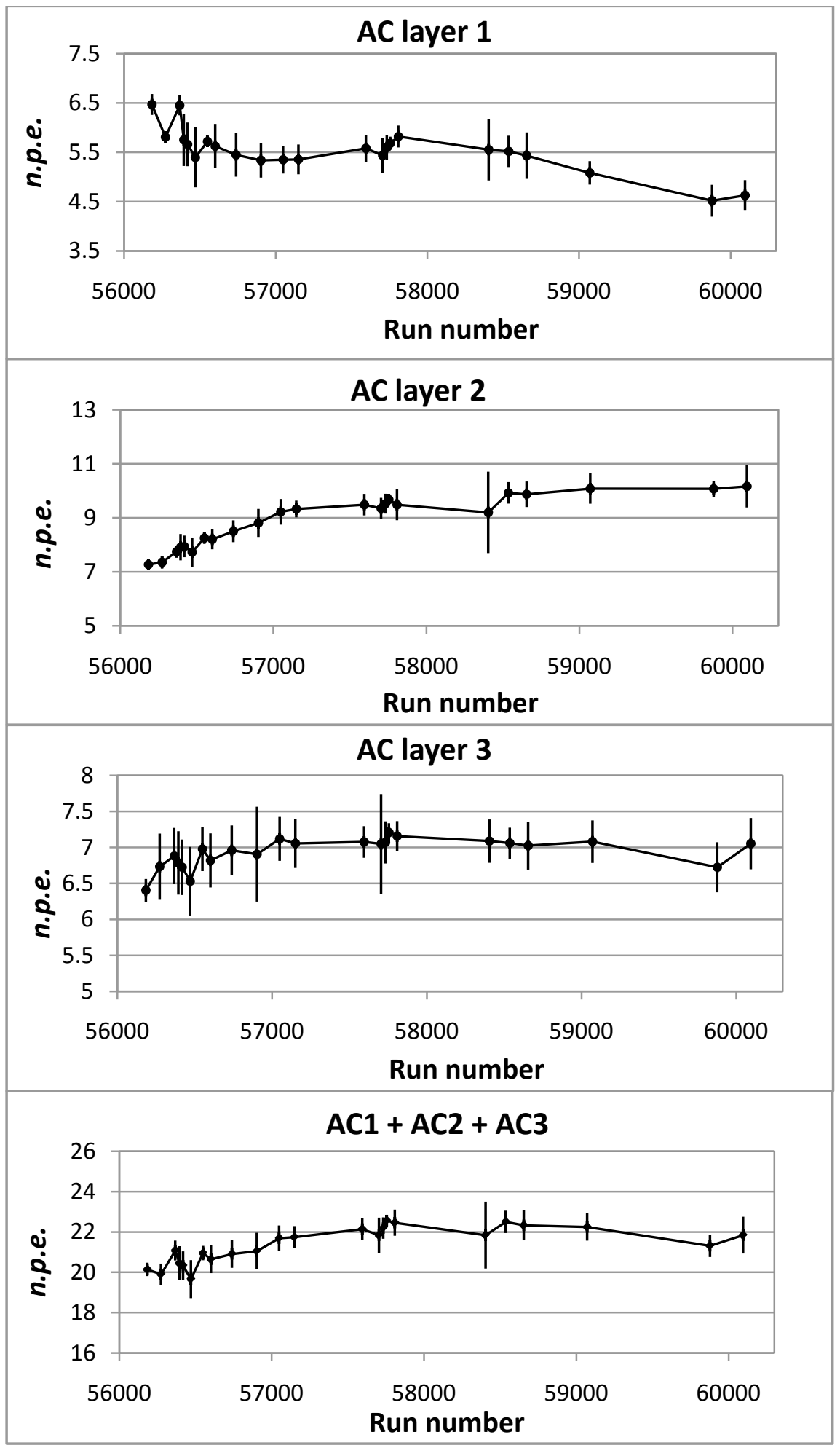

Figure 3.4.15 Number of AC photoelectrons as a function of run number. 


\section{CHAPTER 4}

DATA ANALYSIS

\subsection{Analysis overview}

The analysis of the E01-011 data follows the flow chart shown in Fig. 4.1.1. The frontend electronics signals stored by the CODA DAQ system, together with scalers and control events are marked on the diagram as "RAW DATA". The analysis software, denoted in the chart as "ANALYZER", represents the Hall C analysis code that was substantially modified to include the hardware changes and the introduction of new experimental techniques. The routines, previously used in HNSS, had to be altered to account for a swap of the SOS by a new HKS spectrometer and to accommodate new detectors.

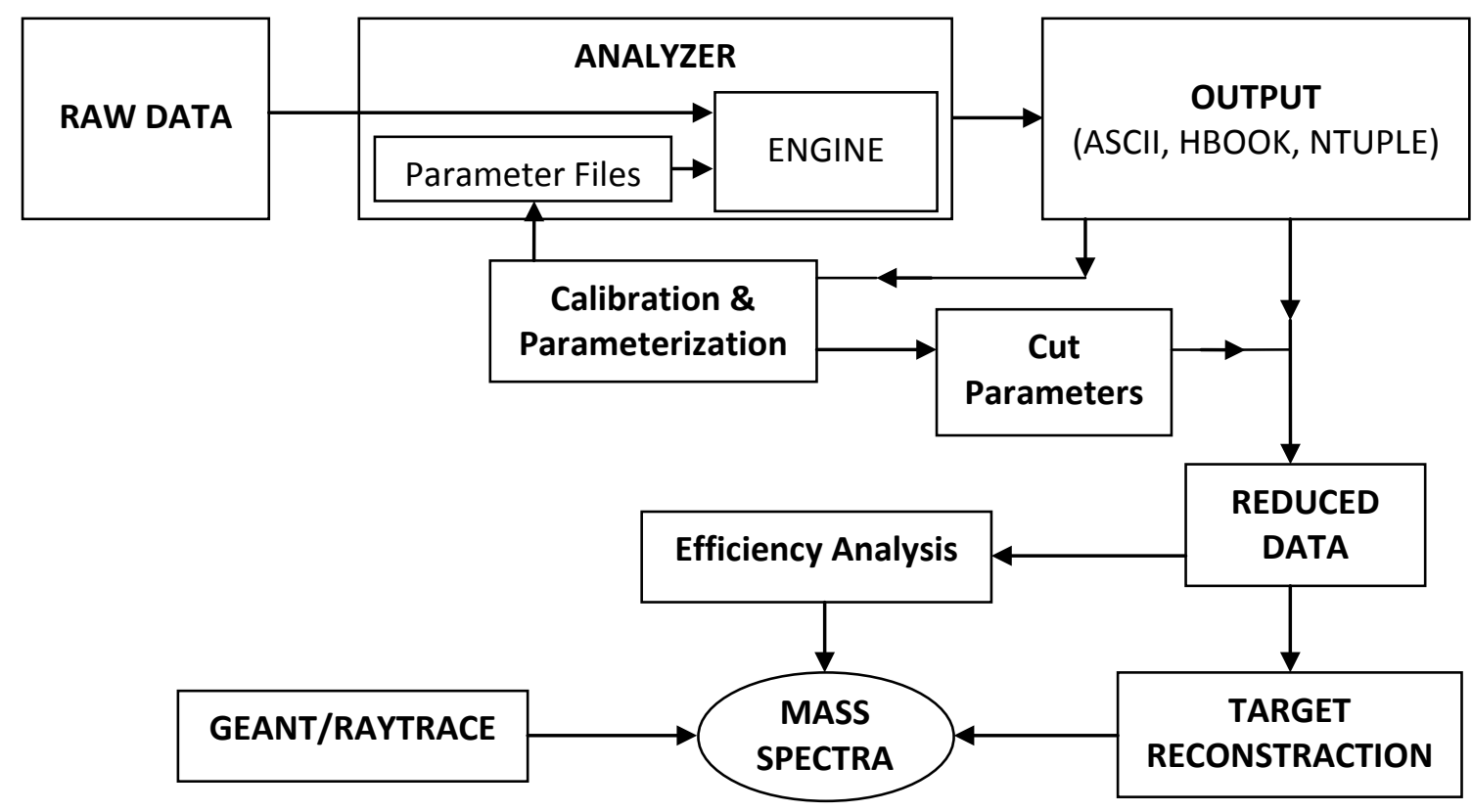

Figure 4.1.1 E01-011 (HKS) data analysis flow chart. 
The raw data inside of the analyzer are fed into the engine. The engine software, Fig. 4.1.2, decodes the electronics signals into variables, reconstructs tracks, identifies particles, and performs physics calculations on an event-by-event basis. The engine starts by reading the configuration file that specifies names of the parameter files to be used in the analysis process. Parameter files contain the flags and a set of variables necessary for calibration procedures, tracking, and PID codes and routines responsible for calculation of the physics quantities.

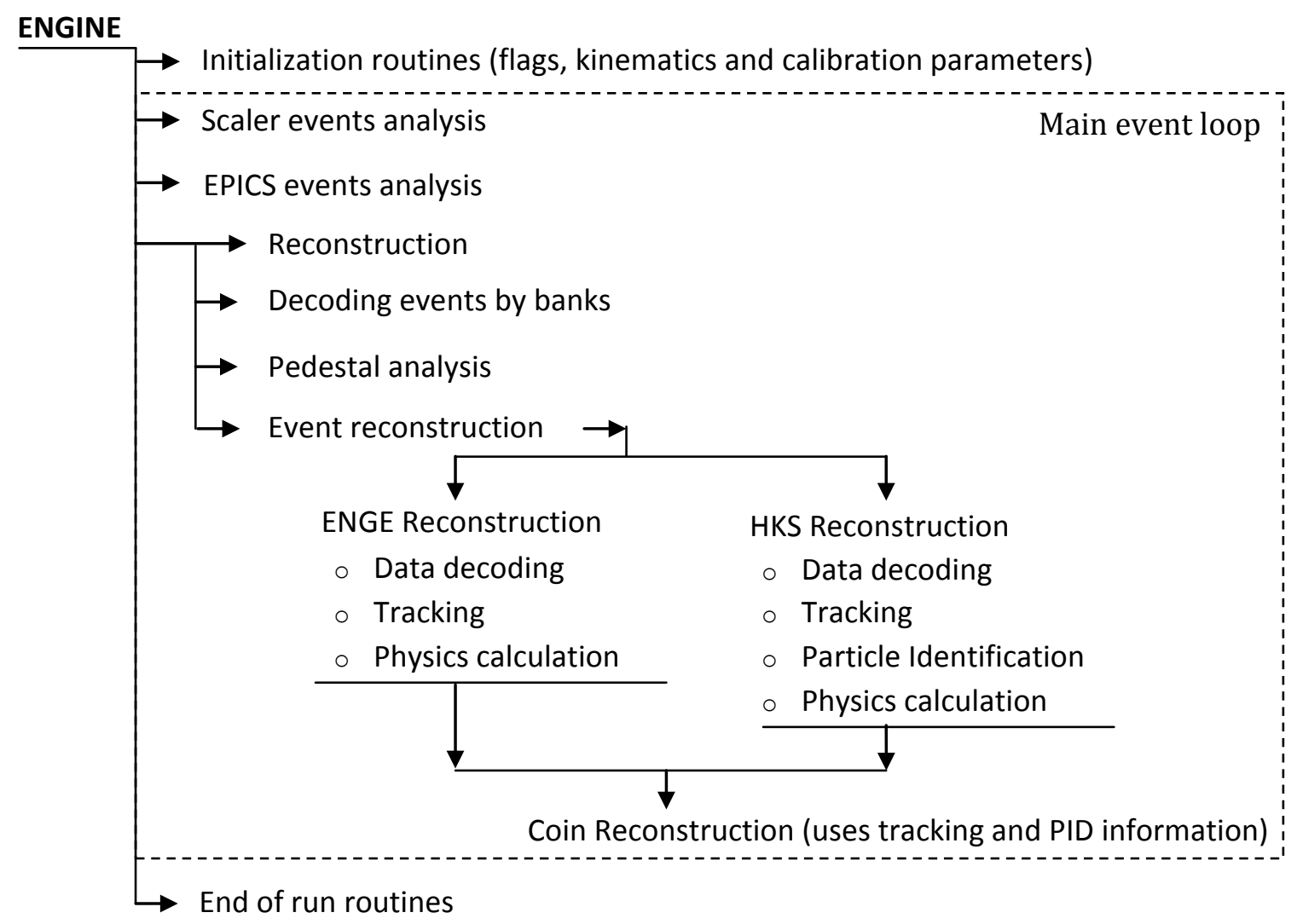

Figure 4.1.2 ENGINE flow diagram.

The engine core is written in FORTRAN. There was also a C-based CEBAF Test Package (CTP) added to the analysis program [73]. It includes parameters required for 
cuts, calibrations and calculations, definitions for histograms and output files. The CTP defines parameters via external ASCII files and does not require any recompilation. That allows the CTP to be a dynamical type of software, and gives a flexibility and an advantage in comparison with the FORTRAN hard-coded engine. The calculation process of physics variables goes through several stages, pointed out in the flow diagram (Fig. 4.1.2). After scaler, EPICS and pedestal analysis, the event reconstruction is performed for both ENGE and HKS spectrometers. Both sides include data decoding, particle tracking, and physics calculations. In addition, the program for the HKS spectrometer includes a PID routine, since there is more than one type of particle in the hadron arm. The engine flow chart finishes by coincidence (coin) reconstruction and endof-run routines. The coin reconstruction calculates coincident quantities using tracking and PID information from ENGE and HKS reconstructions. The end-of-run routines consist of detector efficiency analysis, output histograms, tests, scaler reports, closing routines, etc.

After the analysis procedures, the results were stored in three types of output files: 1) Histograms; 2) Ntuples; 3) ASCII files. The histogram files, written in CERN's HBook format, contain the histograms for detector performance and data integrity check. The Ntuple files consist of many physics and detector variables stored in event-by-event order. The event based representation of the data in Ntuple files makes it easy to perform a sophisticated analysis by introducing limiting cuts on the variables of interest. Such offline analysis is commonly done with the help of programming frameworks PAW and ROOT. The output ASCII-formatted files include the information about hardware and software scalers, integrated beam charge, detector efficiencies, and EPICS signals. The 
calibration of the detectors was done with software customized for each specific detector. The E01-011 was mostly analyzed using the PAW macro language to handle the calibration and further analysis. The results of calibrations were fed back into the analyzer parameter files and then applied in the engine routines. The event selection cuts were formed to eliminate from the data events associated with particles other than kaons, and trajectories outside of the understood acceptance region. Commonly, these are acceptance and PID cuts that result in skimmed data, or "reduced data". Despite the fact that the standard Hall $\mathrm{C}$ engine software included the codes for target reconstruction, the E01-011 collaboration used external routines to reconstruct the target coordinates. The tuning procedure for reconstruction matrices had to be optimized for the new spectrometer (HKS) and the new optical configuration (ENGE tilt). The procedure included a time consuming iteration process that had to be performed outside of the analyzer code on the kaon skimmed data. Having the data analyzed first and further parameterized and then tuned, made it possible to significantly reduce the CPU usage time. The final mass distributions included coincidence cuts that helped to separate the real kaons and accidental background. The details of this analysis will be given later in this chapter. The spectrometer acceptance information extracted from GEANT and RAYTRACE simulations together with efficiencies estimated from the reduced data served for calculation of the cross sections of the hypernuclear states. 


\subsection{HKS data analysis}

\subsubsection{Tracking}

The information read out from the two drift chambers (HDC) is used together with the timing measurement from the scintillators to estimate the particles' trajectories in the spectrometer system. Since the design of the HKS wire chambers was technically similar to those of the standard SOS spectrometer, the standard tracking code was adapted for tracking analysis. The tracking algorithm includes the following key elements: 1) drift time estimate and mapping to drift distance; 2) space points calculation; 3) left-right

ambiguity resolution; 4) contraction of the candidate tracks (stubs); 5) fitting and determination of the final physical track. The drift distance information is essential for estimate of the position in space of the traversing particle.

The hit coordinates are further used in the code to compose the track. To determine the drift distance, the drift time has to be calculated first. For that, the TDC values of the hits at individual wires, $t_{\mathrm{tdc}}$, are used together with the common stop of the TDC that was formed by the trigger from scintillators. The common TDC stop introduces the offset, $t_{\text {start }}$. The timing offsets in the electronics, $t_{\text {trace, }}$ and in the cables, $t_{\text {cable, }}$ are accounted for as well as the propagation time, $t_{\text {prop }}$, of the signal along the hit wire. All these timing quantities together compose the drift time:

$$
t_{d r i f t}=t_{\text {tdc }}+t_{\text {start }}-t_{\text {trace }}-t_{\text {cable }}-t_{\text {prop }} \text {. }
$$


Finally the drift distance is estimated with the help of look-up table. The look-up table was obtained periodically on a several day interval.

The tracking algorithm works by grouping the crossed wires, for example wires from $x$ and $u$ planes, into pairs. Further, the code loops over pairs and groups the pairs into combinations. The nearest wire combinations are tested with the so-called "space point" criterion that requires the square root of the sum of the squared distances between wire intersections to be confined within a set parameter. One hit pair combination is enough to recognize a space point. Later, the left-right ambiguity is resolved for each wire in the space point by comparing drift patterns and drift distances from two planes with parallel wires that are offset by half a cell size. For the tracking algorithm to work properly at least five out of six planes are required to have a hit. In addition the total number of hits in one chamber is limited to 40 . The obtained space points for each chamber are fitted with miniature tracks, called "stubs". Stubs are generated for each drift chamber individually. The positions and slopes are used to match the approximately collinear stubs and form a physical track. The specific stub criteria that consist of the vertical and horizontal distance and slope ranges, $\left(x, x^{\prime}\right)$ and $\left(y, y^{\prime}\right)$, selects the area where two stubs have to lie in order to be a part of the same track. In the case of multiple tracks, $\chi^{2}$ minimization is applied for selection of the best track. Multiple track events are relatively rare $(<0.1 \%)$ and are mostly caused by multiple hits and noisy wires. To ensure the validity of the track, the tracking algorithm requires at least five out of six planes to fire for each chamber. Once the track is found, its focal plane quantities are determined. The $x$ and $y$ positions together with the vertical and horizontal slopes respectively comprise the focal plane variables: $x_{f p}, y_{f p}, x^{\prime}{ }_{f p}, y^{\prime}{ }_{f p}$. 


\subsubsection{Event reconstruction}

Using the focal plane quantities one has to project the trajectory from the focal plane back to the reaction vertex in the target. Such a transformation from focal to target plane is needed to obtain the reaction's momenta and emission angles, which cannot be directly measured in the experiment. The procedure is done with the help of a reconstruction routine, which estimates the relative particle momentum $\delta=\left(p-p_{0}\right) / p_{0}$ together with the position and tangent of angles in the horizontal (scattering) and vertical (dispersive) planes. The vertical position on the target, $y_{t a r}$, is assumed to be zero. Depending on whether the beam is unrastered or rastered, $x_{\text {tar }}$ is assumed to be zero or determined from the raster magnet current readback, respectively. Thus, only three variables need to be

reconstructed: $\delta, x_{t a r}^{\prime}$, and $y_{t a r}^{\prime}$. If the reconstructed target variables are denoted by $q_{t a r}^{i}$, then:

$$
q_{\text {tar }}^{i}=\sum_{j, k, l, m=0}^{N}\left(M_{j k l m}^{i}\right) x_{f p}^{j} x_{f p}^{\prime k} y_{f p}^{l} y_{f p}^{\prime m}
$$

The indices are restricted by the order $N$ of the transformation:

$$
1 \leq j+k+l+m \leq N
$$

In this analysis we used a matrix $M_{j k l m}^{i}$ with coefficients up to 6-th order. Each target variable has its own reconstruction matrix elements. The reconstruction is basically carried out by a Taylor expansion of the solutions of the equations of motion in the 
magnetic spectrometer system, i.e. $q_{t a r}=f\left(x_{f p}, x_{f p}^{\prime}, y_{f p}, y_{f p}^{\prime}\right)$. Matrix formalism used in reconstruction analysis is described in detail in reference [74].

The elements of the matrix are determined in an iterative process. The first approximation of the matrix was found with the help of RAYTRACE simulations for the ENGE and HKS arms together with the GEANT Monte Carlo. Further iterations include the application of the collimator plates, called sieve slits. The sieve slit (SS) is a plate with an array of holes. In order to match the slits with the particle trajectories the holes are not perpendicular to the slit plane. Each hole allows selecting events with specific horizontal and vertical angles. In such way the SS serves primarily for optimization of the $x_{t a r}^{\prime}$ and $y_{\text {tar }}^{\prime}$ reconstruction. In the experiment we used two ENGE and HKS sieve slits made of a tungsten alloy. Both were located behind the splitter magnet with respect to the incident beam. The ENGE SS is positioned in front of the ENGE entrance and HKS SS is placed in front of the first quadrupole magnet of the HKS arm. Because the beam passes first through the splitter magnet before it reaches both sieve slits, the angles measured by reconstructing holes are not the original reaction angles, but the ones affected by the splitter magnet. Therefore the complete focal plane to target reconstruction has to include two steps: 1) transformation from focal plane to the SS plane; 2) further transport from the SS plane to target. The final target reconstructed angle variables are represented as a function of the position coordinates of the holes on sieve slits and relative momenta, $q_{\text {tar }}^{\prime}=f\left(x_{s s}, y_{s s}, \delta\right)$. The RAYTRACE simulation is used for SS to target transport. On the ENGE side, as a result of the tilt method, RAYTRACE is not suitable and a GEANT simulation is used instead. The SS runs are taken separately for ENGE and HKS arms. 
For that, the ${ }^{12} \mathrm{C}$ target is used without a beam raster in order to fix the beam position $x_{\text {tar }}$ and $y_{\text {tar }}$ on the target. The HKS and ENGE sieve slits are shown in Fig. 4.2.1. Their geometrical properties are summarized in the Table 4.2.1.

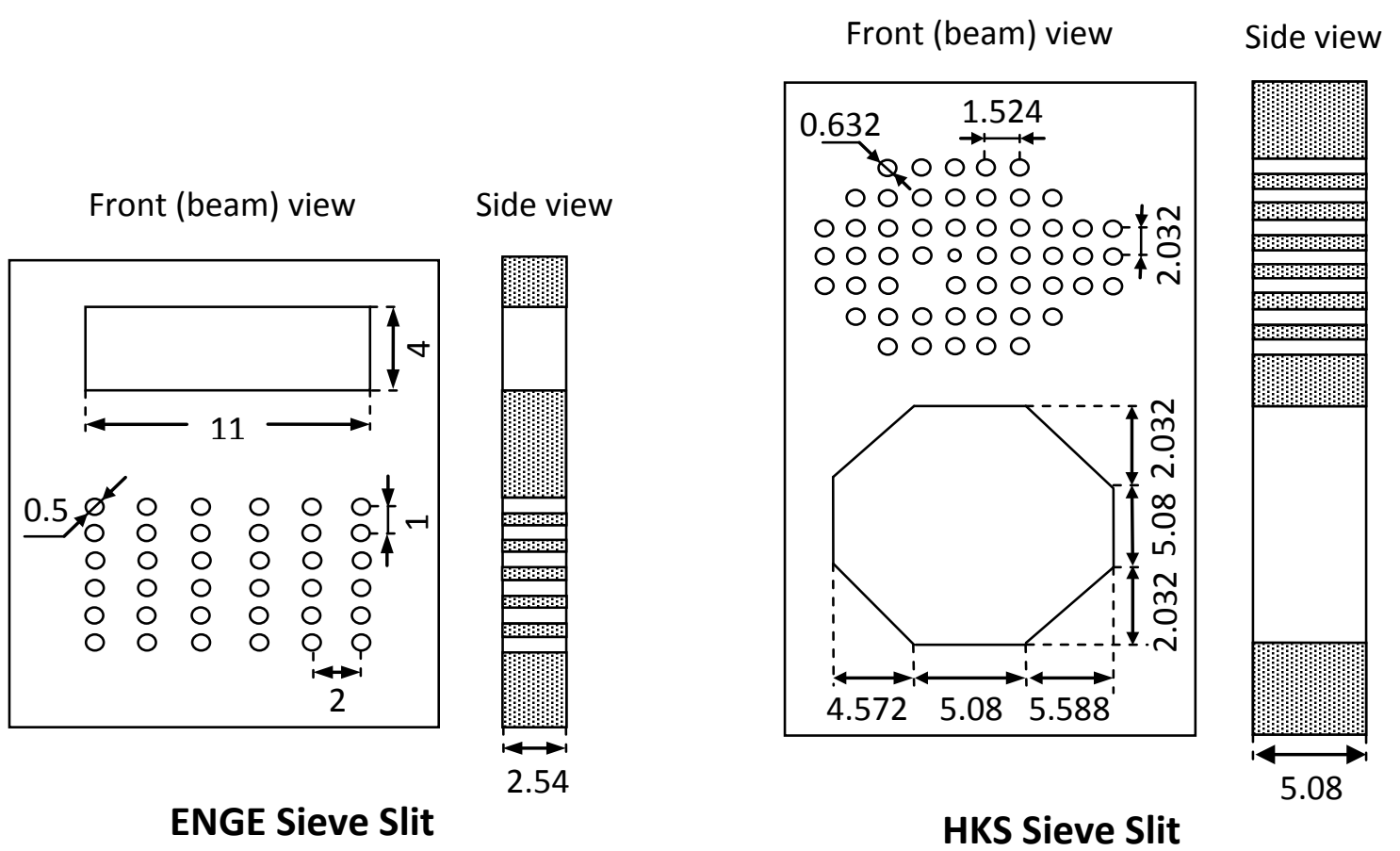

Figure 4.2.1 ENGE and HKS sieve slit collimator plates. The units are in $\mathrm{cm}$.

Table 4.2.1 Geometrical parameters of the ENGE and HKS sieve slits.

\begin{tabular}{lccccccc}
\hline Plate & $\begin{array}{c}\text { \# of } \\
\text { holes }\end{array}$ & $\begin{array}{c}\text { Hole geometry } \\
(\text { column } \times \text { rows })\end{array}$ & $\begin{array}{c}\text { Hole diameter } \\
(\mathrm{cm})\end{array}$ & $\begin{array}{c}\text { Holes spacing }(\mathrm{cm}) \\
\text { horizontal }\end{array}$ & vertical & Symmetrical \\
$(\mathrm{y} / \mathrm{n})$ & $\begin{array}{c}\text { Plate width } \\
(\mathrm{cm})\end{array}$ \\
\hline ENGE & 30 & $6 \times 5$ & 0.5 & 2.0 & 1.0 & Yes & 2.54 \\
\hline HKS & 53 & $10 \times 7$ & 0.632 & 1.524 & 2.032 & $\begin{array}{c}\text { No } \\
\text { (One hole with } \varnothing 0.318 \mathrm{~cm} \\
\text { and one hole missing) }\end{array}$ & 5.08 \\
\hline
\end{tabular}




\subsubsection{Time of flight measurements}

The scintillating hodoscopes serve to calculate the time of flight (TOF) of the charged particles. In the HKS arm we employ the HTOF1X and HTOF2X hodoscope layers. The TOF, expressed in terms of the relative velocity $\beta$, is calculated on a track-by-track basis. To estimate $\beta$, we only use scintillator hits which are along a certain track. The rest of the hits are discarded to eliminate the noise, although they might be considered for another track. A valid $\beta$ requires at least two scintillators, one from each layer, to have fired along the track. By calculating the hit position on the scintillating pad and measuring the time of the particle's arrival, one can calculate the relative velocity. In order to achieve the required resolution, three corrections need to be applied to the recorded raw TDC timing information:

1) The propagation time within the scintillator, which depends on the distance between the hit point and the collecting PMT, and the velocity of light in the paddle, $t_{\text {prop }}=d_{\text {prop }} / v_{\text {scin }}$. Since the light does not propagate straight in the paddle but experiences multiple internal reflections, the effective velocity of light is a function of both the dimensions of the paddle and the index of refraction of the scintillating material. The time propagation corrections can reach values up to 10 ns.

2) The time walk associated with the use of leading edge discriminators. The ADC signal represents a time-integrated signal, while the arrival time is measured by a TDC, which is stopped when the signal exceeds the discriminator threshold. 
Large signal will fire the discriminator earlier than a small one, even though both signals may have the same mean fire value as shown in Fig. 4.2.2.

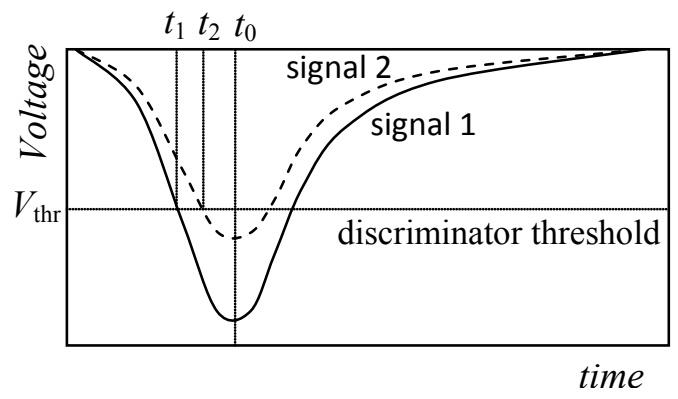

Figure 4.2.2 Time-walk effect due to a size variation of the signal.

To account for the time walk, a pulse height correction is needed, which is determined from the correlation between $\mathrm{ADC}$ and TDC signals. The voltage versus time profile is described by Lorentzian distribution. Further, the FWHM is extracted and used in the calculation of the time walk. Then the pulse height correction is estimated in the form:

$$
t_{\mathrm{phc}}=P_{1} / \sqrt{A D C-P_{2}},
$$

where $P_{1}$ and $P_{2}$ are the constants found by fitting the ADC - TDC correlation data.

3) The third correction has to be done because of the mismatch in the cable length and electronic delays. These time offsets are determined by comparing pairs of scintillators oriented perpendicular to each other. The time of flight, $t_{\text {mean }}$, is measured by the mean time of two scintillators and the theoretical value, $t_{\text {calc }}$, is estimated from the knowledge of particle's velocity and the distance between the 
scintillating pads. Then the timing offset, $t_{\text {off }}$, is extracted by minimizing the difference between measured and calculated time quantities $\Delta t=t_{\text {mean }}-t_{\text {calc }}$.

After all corrections are found the corrected time of flight measured by PMT becomes:

$$
t_{\text {pmt.corr. }}=t_{\text {pmt }}-t_{\text {prop }}-t_{\text {phc }}-t_{\text {off }} \text {. }
$$

This procedure is done for each PMT on event by event basis.

\subsubsection{Particle Identification}

\subsubsection{Standard approach}

The standard approach to particle identification is to apply a sequence of limiting cuts on particular variables that allow distinguishing between different particles. The PID is performed only in the HKS arm, where the differentiation between three particles, protons, kaons, and pions, is required. Despite the fact that the ENGE arm detects both recoil electrons and negative pions, particle differentiation between $e^{\prime}$ and $\pi^{-}$is not needed. The rate of the $\pi^{-}$particles in comparison to that of electrons is extremely small, which allows us to neglect them.

The standard PID routine employs the calibrated ADC signals from aerogel and water Cherenkov detectors as well as relative velocity (beta) distributions. Figure 4.2.3 shows an AC spectrum, calibrated in the number of photoelectrons (n.p.e.). The left peak, centered around zero is the pedestal. It contains protons and kaons. The distribution on the right represents the pions. At the kinematics setting of our experiment $\left(p_{0}=1.2 \mathrm{GeV}\right)$, 
the AC counters are only sensitive to pions, hence the kaons and protons are undetected and show up in the pedestal area. All three AC layers have a very similar n.p.e. distribution. According to the standard PID procedure, to separate kaons and protons from pions we have to introduce a cut between the particles, as displayed on the figure.

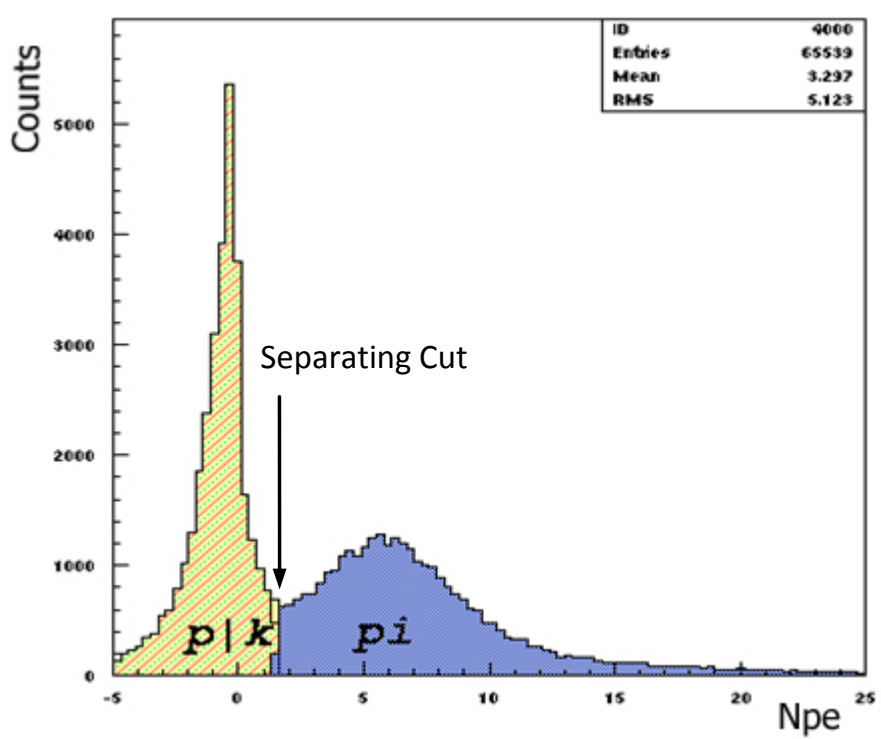

Figure 4.2.3 Schematics of the particles location in typical AC n.p.e. spectrum.

In the E01-011 analysis the kaon selection in the AC distributions was done by placing only one cut on the summed $\mathrm{AC}$ signal, i.e. $\mathrm{AC}_{1}+\mathrm{AC}_{2}+\mathrm{AC}_{3}$. For example, $\left(\mathrm{AC}_{1}+\mathrm{AC}_{2}+\mathrm{AC}_{3}\right)<6$, is the cut applied to the $\mathrm{AC}$ detectors for the ${ }^{12} \mathrm{C}$ target data.

To further separate the protons from kaons, the WC n.p.e. distributions are used. Figure 4.2.4 displays the location of the particles in the typical WC n.p.e. distribution. The real particle distributions are described by Poisson statistics. The position of the protons, kaons, and pions on the picture is displayed schematically. The separating cut has to be placed somewhere between protons and kaons, as it is shown on the figure 
(arrow). Similar to the AC counters, there is only one cut utilized for WC detectors, $\left(\mathrm{WC}_{1}+\mathrm{WC}_{2}\right)>75$ (in the case of ${ }^{12} \mathrm{C}$ target).

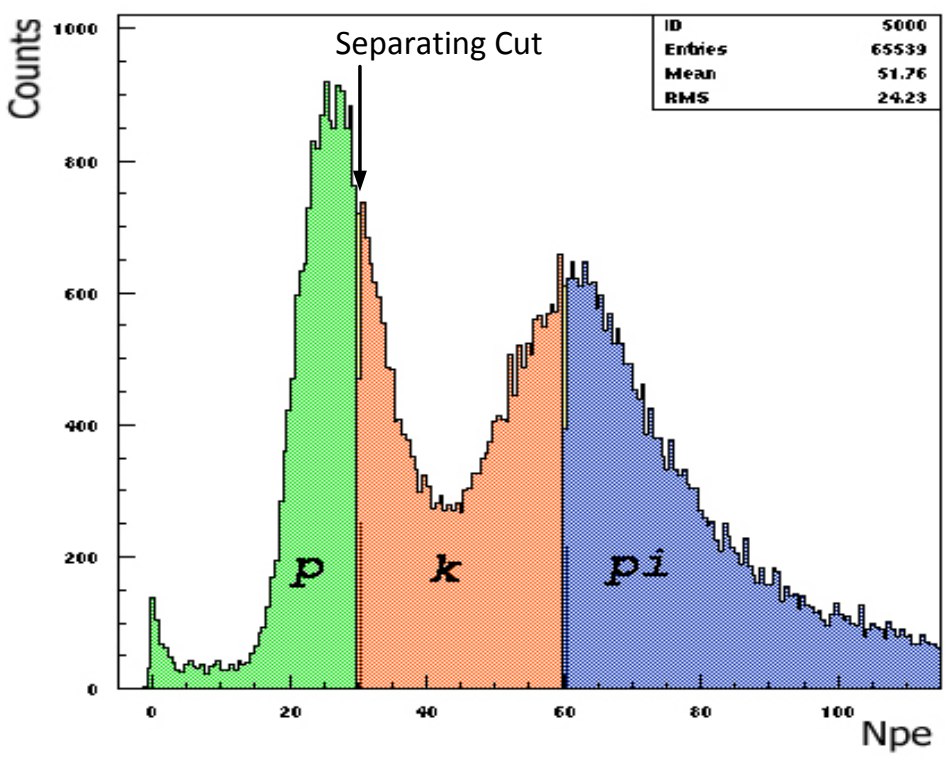

Figure 4.2.4 Schematics of the particles location in typical single layer WC n.p.e. spectrum.

The final cut used in the standard PID technique is a limiting cut on the beta distribution, shown in Fig. 4.2.5. Again the figure schematically displays the expected position of the particles. The following cut, $\left|\beta-\beta_{K}\right|<0.06$, has been used in the analysis to identify a kaon. Here, the $\beta_{K}$ is calculated from the reconstructed momentum assuming a kaon mass, while $\beta$ is obtained from a TOF measurement. 


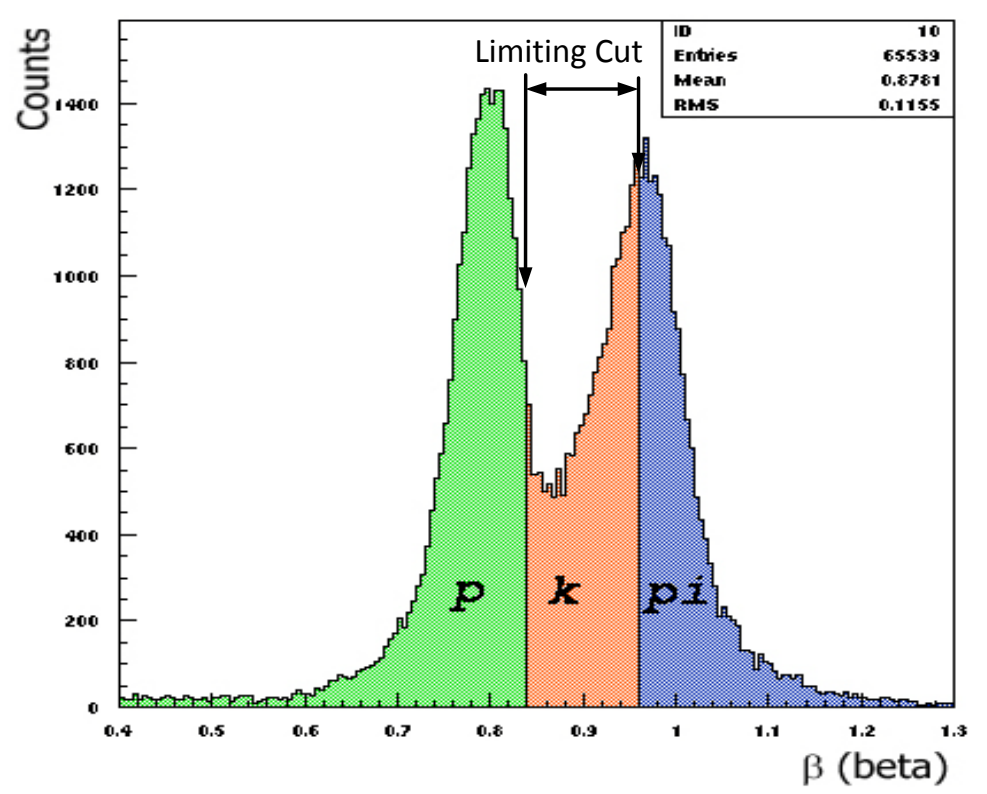

Figure 4.2.5 Schematics of the particles location in the beta spectrum.

The "AND" combination of all three cuts, $\mathrm{AC}, \mathrm{WC}$ and $\beta$, defines the standard kaon identification:

$$
\left(\mathrm{AC}_{1}+\mathrm{AC}_{2}+\mathrm{AC}_{3}\right)<6 \&\left(\mathrm{WC}_{1}+\mathrm{WC}_{2}\right)>75 \&\left|\beta-\beta_{K}\right|<0.06
$$

\subsubsection{Likelihood approach}

\subsection{Overview of the method}

The standard PID approach described above may result in the loss of good events when the individual PID distributions have strongly overlapping peaks. There could be a situation where an event that satisfies all but one condition. For example, an event shows up as a kaon in the AC and WC counters but appears outside of the limiting cut in the 
beta spectrum. The event has a high probability of being a kaon but does not get identified as such. The cuts can be altered, however, cuts that are too loose result in large backgrounds while over-tight cuts reduce the efficiency for detection of the particle of interest. The reduced yield increases the uncertainty on the extracted cross-section values. For a reliable hypernuclear spectroscopy, the statistical significance of the physics peaks is very important. It is worth investigating an alternative to the standard hard-cut PID method that can eliminate the loss of good events. Our new approach to PID employs the likelihood method for a decision on the particle's identity. The likelihood method is commonly used in high-energy physics, but is not widely employed in our nuclear physics community. According to the likelihood PID method, we assign probability density functions (pdf) to each of the Cherenkov detector n.p.e. spectra and relative velocity (beta) distributions for every particle present. From these we compose normalized likelihoods for each of the three particles. The proposed method, in our estimation, is expected to reduce background in the missing mass spectrum, increase signal-to-noise ratio, and maximize the resolution of the core excited states peaks.

We have split the likelihood PID analysis into several steps:

1) Step 1: Choose the PID variables.

2) Step 2: Perform hard cut PID analysis to obtain "clean" distributions.

3) Step 3: Parameterize "clean" distributions and store results as normalized pdfs.

4) Step 4: Combine obtained pdfs into final likelihood values.

The flow diagram of the process is shown in Fig. 4.2.6. 


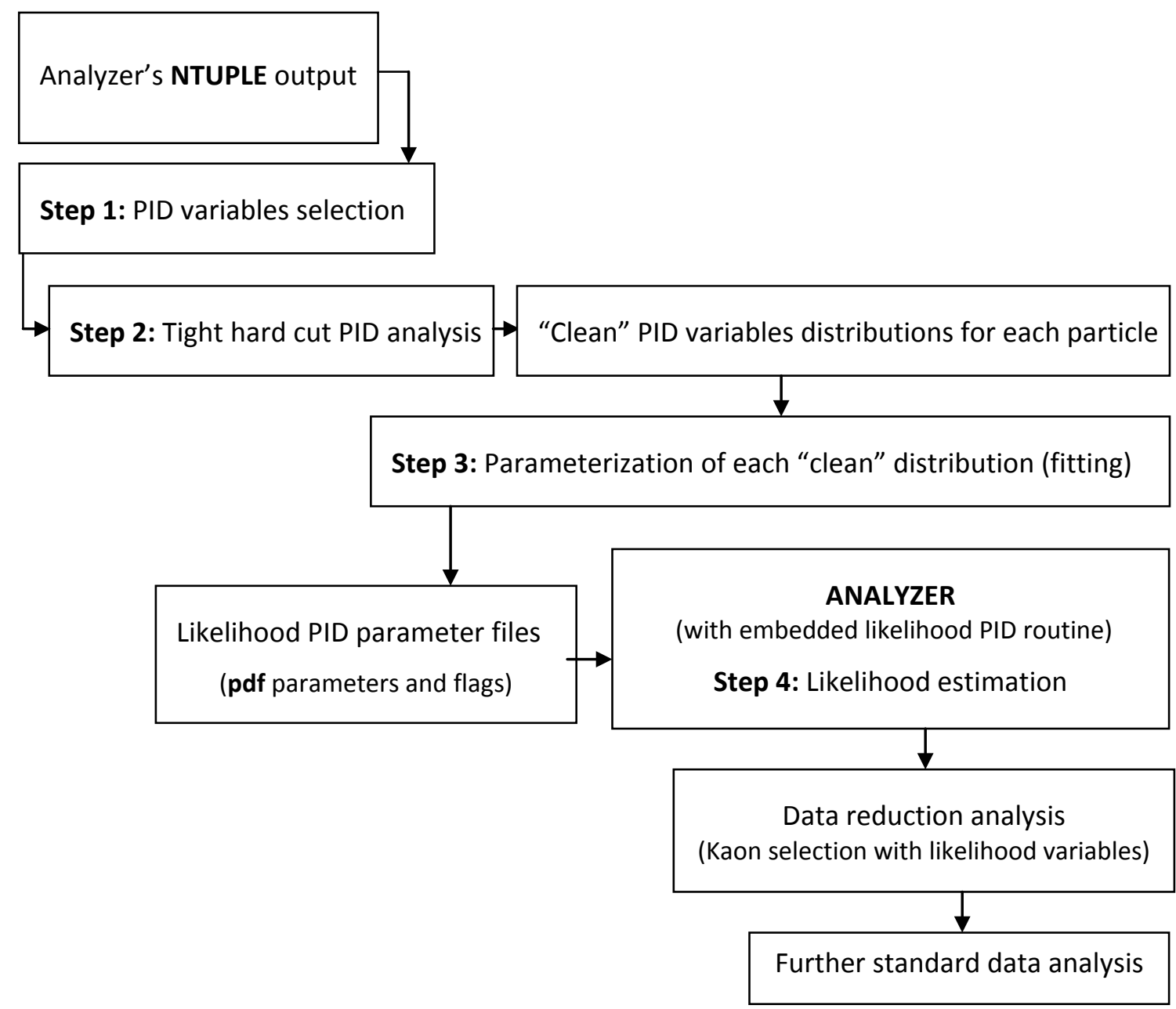

Figure 4.2.6 Schematic diagram of likelihood PID analysis.

\subsection{Selection of the PID variables}

The PID variables are chosen from the calibrated detector signals. The main criterion of selection is based on the resolution of the signal, which, after some analysis, may allow seeing background free single-particle distributions. Three layers of aerogel and two layers of water Cherenkov detectors together with the TOF measurements provide six independent variables for PID analysis. Both types of employed Cherenkov detectors, i.e. 
$\mathrm{AC}$ and $\mathrm{WC}$, perform differently along the focal plane. In case of the aerogel Cherenkov detectors pdfs were created for all 21 segments while for the water Cherenkov it was sufficient to divide the 24 segments into six groups defined by the particle momentum. Together with the one independent TOF measurement this required $(21+6+1)=28 \mathrm{pdf}$ variables for each individual particle. Table 4.2.2 shows the variables used in likelihood PID analysis.

Table 4.2.2 List of pdf variables per particle used in the likelihood PID analysis.

\begin{tabular}{lcc}
\hline PID variables & \# of pdfs & $\begin{array}{c}\text { \# of independent } \\
\text { pdfs }\end{array}$ \\
\hline $\begin{array}{l}\text { Three aerogel Cherenkov detectors }(7 \text { segments in each counter) } \\
3 \text { statistically independent variables: } \mathrm{AC}_{1}, \mathrm{AC}_{2}, \mathrm{AC}_{3}\end{array}$ & $3 \cdot 7=21$ & 3 \\
\hline $\begin{array}{l}\text { Two water Cherenkov detectors }(12 \text { segments in each counter) } \\
2 \text { statistically independent variables: } \mathrm{WC}_{1}, \mathrm{WC}_{2}\end{array}$ & 6 & 2 \\
\hline $\begin{array}{l}\text { Beta variables } \\
\text { Beta extracted from TOF calculations (hodoscopes): } \beta\end{array}$ & 1 & 1 \\
\hline
\end{tabular}

\subsubsection{3 "Clean" distributions and parameterization}

The "clean" distributions are obtained from the selected likelihood PID variables by placing restrictive hard cuts. These cuts yield several distributions with each of them corresponding to a separate particle. For example, the variable of $\mathrm{AC}$ layer $1, \mathrm{AC}_{1}$ can have three distributions: $\mathrm{AC}_{1 \mathrm{p}}, \mathrm{AC}_{1 \mathrm{~K}}, \mathrm{AC}_{1 \pi}$.

In comparison with the standard PID cuts, the cuts used in this parameterization analysis are extremely restrictive in order to obtain the cleanest possible spectrum for a single type of particle with a minimum amount of contamination. Since this comes at the 
expense of statistics, one needs to analyze a large amount of data files, linked together to compensate for the reduced statistics.

\section{Aerogel Cherenkov}

In order to obtain a "clean" kaon distribution for $\mathrm{AC}_{1}$ we have to select only kaons by placing hard cuts on $\mathrm{AC}_{2}, \mathrm{AC}_{3}, \mathrm{WC}_{1}, \mathrm{WC}_{2}$, and beta. For $\mathrm{AC}_{1 \mathrm{p}}$ and $\mathrm{AC}_{1 \pi}$ the mentioned variables must have proton and pion selective cuts. We find "clean" proton, kaon, and pion distributions for each segment in each $\mathrm{AC}$ layer. A segment distribution is the summed n.p.e. signals from top and bottom photomultiplier tubes.
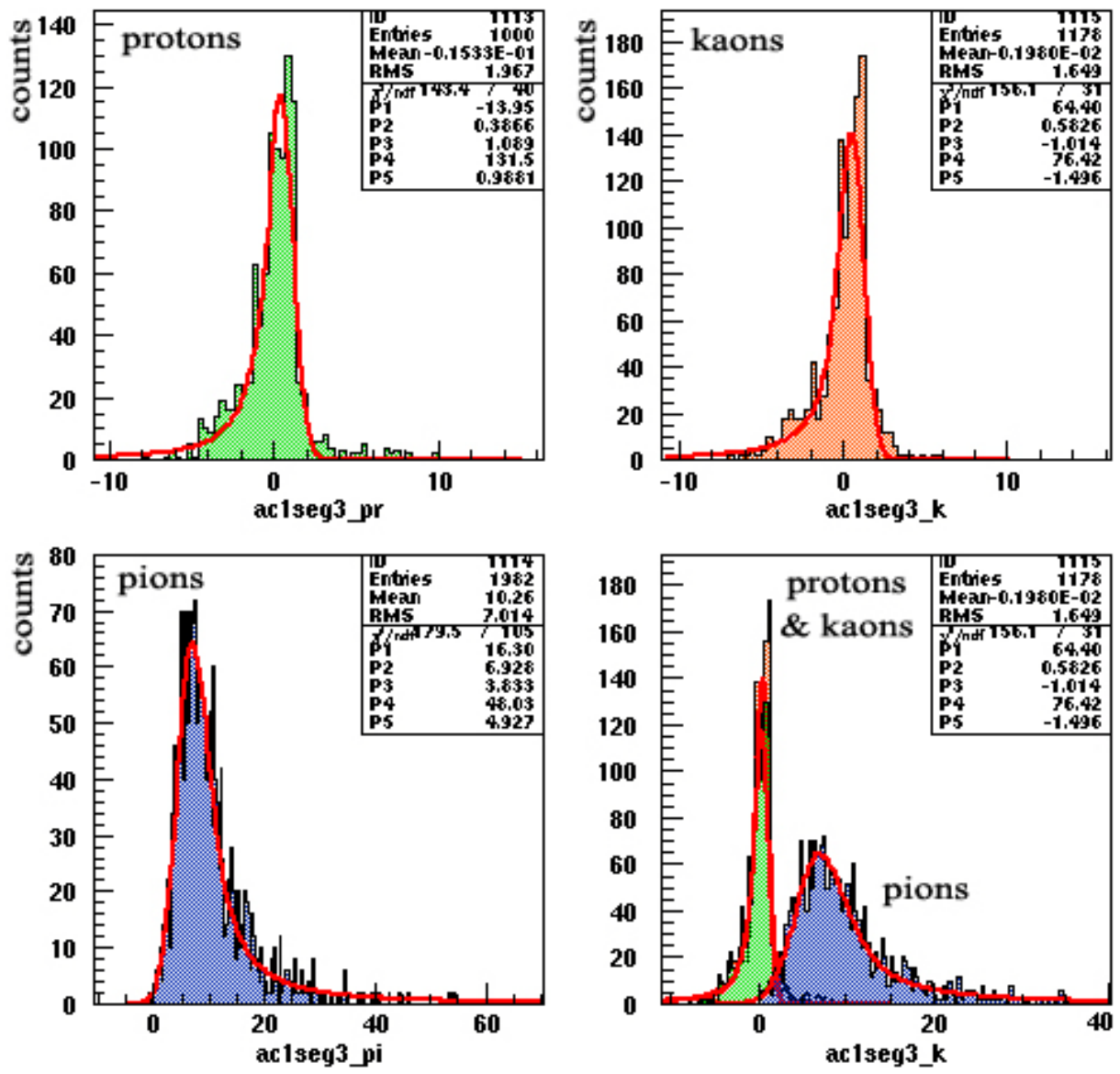

Figure 4.2.7 Example of the AC1 segment 3 "clean" proton, kaon, and pion n.p.e. distributions after applying hard PID cuts. 
Figure 4.2.7 displays an example of the hard cut PID result for AC layer 1, segment 3 . Very similar distributions can be seen in the other $6+7+7=20$ segments. To obtain these distributions restrictive cuts were applied on the beta, aerogel and water Cherenkov n.p.e. spectra. Detected by AC detector pions form a wide peak, depicted in blue color on the figure. The protons and kaons are not detected by the AC counters and, therefore, show up in the pedestal area of the AC n.p.e. spectra shown by the green and red distributions.

Before going to the next step, which is the parameterization of the "clean" distributions, we have to decide which AC n.p.e. spectra actually have to be parameterized. Since the AC counter is not detecting the protons and kaons, we have to make sure that our AC pdf functions do not differentiate between these particles. For that, we have to use one pdf function for both, kaons and protons. In our analysis we decided to fit only proton $\mathrm{AC}$ n.p.e. distributions and assumed the kaon distributions to be the same. The preferential choice of protons was motivated by the reason that there are many more protons available in the production data than kaons. The fraction of the kaons ranges from 5 to $10 \%$, while the number of pions and protons are approximately equal.

The next step is to find functional parameterizations for the pdfs by applying an appropriate fit to each of the n.p.e. plots. Because the distributions for protons and kaons are chosen to be the same, we only have to parameterize $2 \cdot 7 \cdot 3=42$ signals. As we can see, the distributions that we have to fit have an asymmetric profile and cannot be described by either Gaussian or Poisson functions. A pseudo-Voigt function - modified to accommodate our case - was found to work the best. The classic pseudo-Voigt function is a combination of Gaussian and Lorenzian, and it is written in the form 


$$
\text { Voigt }=p_{1} \cdot \exp \left(-\frac{\left(x-p_{2}\right)^{2}}{p_{3}^{2}}\right)+p_{4} \cdot\left(1+\frac{\left(x-p_{2}\right)^{2}}{p_{5}^{2}}\right)^{-1}
$$

Here $p_{2}$ is the mean value $(\mu)$ and $p_{3}$ and $p_{5}$ determine the width of the Gaussian and Lorenzian parts of the distribution, respectively. This function is suitable for distributions with wide tails. However, in such a form it has a symmetrical shape. Therefore, a modification is needed to create an asymmetry in the function. We have defined the following functions:

1) Left Voigt (asymmetrical): pseudo-Voigt function on the left side of peak's mean value and Gaussian on the right side:

$$
\text { LeftVoigt }=\left\{\begin{array}{l}
\left(p_{1}+p_{4}\right) \cdot \exp \left(-\left(x-p_{2}\right)^{2} / p_{3}^{2}\right), \text { if } x>p_{2} \\
p_{1} \cdot \exp \left(-\left(x-p_{2}\right)^{2} / p_{3}^{2}\right)+p_{4} /\left(1+\left(x-p_{2}\right)^{2} / p_{5}^{2}\right), \text { if } x \leq p_{2}
\end{array}\right.
$$

2) Right Voigt (asymmetrical): Gaussian on the left side of the peak's mean value and pseudo-Voigt on the right side:

$$
\text { RightVoigt }=\left\{\begin{array}{l}
p_{1} \cdot \exp \left(-\left(x-p_{2}\right)^{2} / p_{3}^{2}\right)+p_{4} /\left(1+\left(x-p_{2}\right)^{2} / p_{5}^{2}\right), \text { if } x \geq p_{2} \\
\left(p_{1}+p_{4}\right) \cdot \exp \left(-\left(x-p_{2}\right)^{2} / p_{3}^{2}\right), \text { if } x<p_{2}
\end{array}\right.
$$

In accordance with this definition we will refer later in the Chapter to the symmetrical Voigt function (Eq. 4.2.7) as Central Voigt.

The proton/kaon n.p.e. distributions have been fit with the Left Voigt, while the pion spectrum used the Right Voigt function. The five parameters $p_{1}-p_{5}$ together with the total number of events (parameter $p_{6}$ ) were saved in a parameter file. Further, the 
functions were normalized to one, Func $c_{\text {norm }}=$ Func $/ p_{6}$. As an example, the fits for AC layer 1 segment 3 are shown in Fig. 4.2.7, and the normalized proton, kaon, and pion pdf functions are presented in the Fig. 4.2.8.

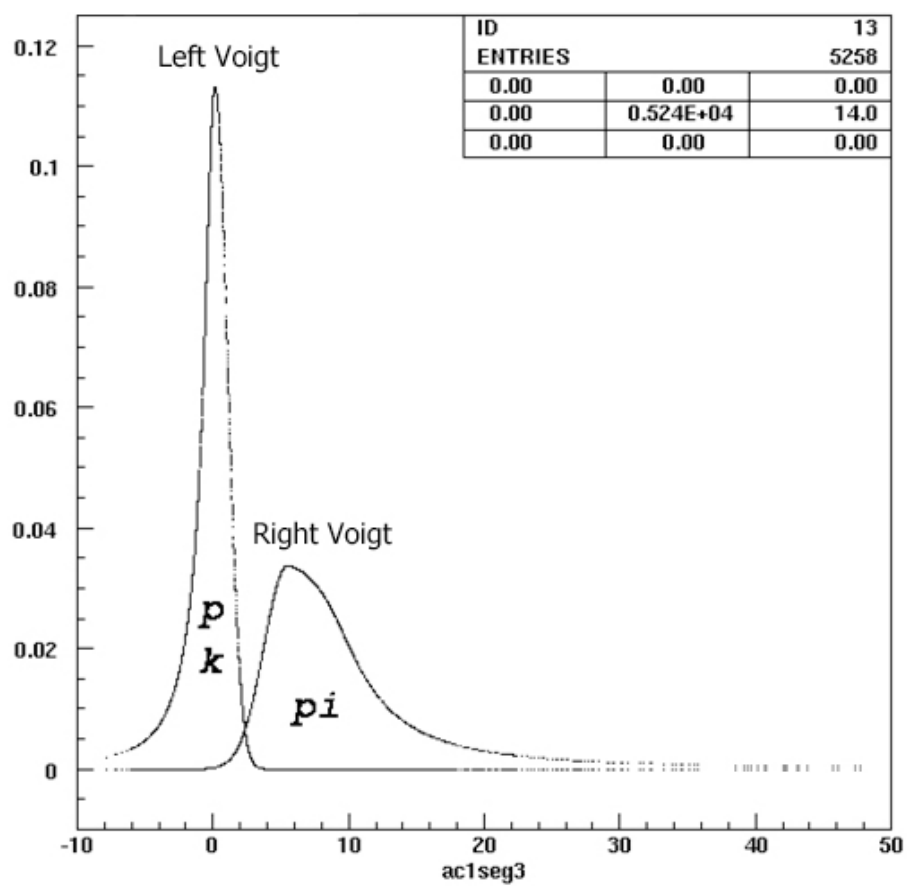

Figure 4.2.8 The normalized AC1_seg3 probability density function (pdf) used in the likelihood PID analysis.

Because of the particle trajectories' angular distribution in the AC detector plane, more than one segment can be hit (Fig.4.2.9). Also, tracking inaccuracies and multiple scattering lead to ambiguity in identifying the segment that has been hit by a certain trajectory. Up to, but no more than two adjacent segments need to be considered. 


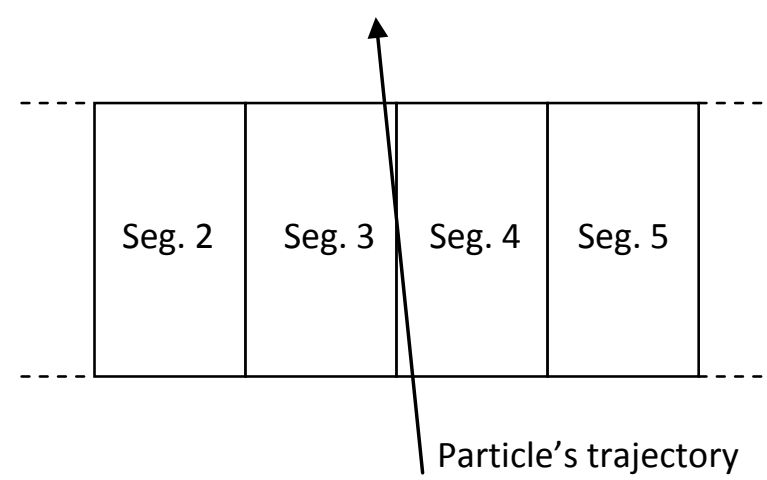

Figure 4.2.9 Possible particle's trajectory through two neighboring segments in AC detector.

The standard tracking routine use only the AC segments that overlap with the particles' trajectory. Inside of the routine, the geometrical coordinates of each segment are found as: $x_{\max }=x_{\text {segc }}+w_{\text {seg }} / 2$ and $x_{\min }=x_{\text {segc }}-w_{\text {seg }} / 2$, with $x_{\text {segc }}$ the center and $w_{\text {seg }}$ the width of the segment (Fig. 4.2.10). Further, the track is projected on the AC detector layer as:

$$
x_{A C_{L}}=x_{f p}+z_{A C_{L}} \cdot x_{f p}^{\prime} .
$$

In the expression above $z_{A C_{L}}$ is the distance from the focal plane to $A C_{L}(L=1,2,3)$ detector along the $z$ axis, $x_{f p}$ and $x_{f p}^{\prime}$ are the $x$ coordinate and the tangent of the angle at the focal plane, respectively.

Then a test is performed for each segment in the AC layer, $x_{\min }-5 \mathrm{~cm} \leq x_{A C_{L}} \leq x_{\max }+5 \mathrm{~cm}$. The additional $\pm 5 \mathrm{~cm}$ account for track misspointing due to inaccuracies in tracking and multiple scattering. A segment which passes this test belongs to the particle's track and therefore is counted. The rest of the segments are 
ignored. Especially at high rate, this standard procedure might lead to the inclusion of neighboring trajectories to the $\mathrm{AC}$ information of the track of interest.

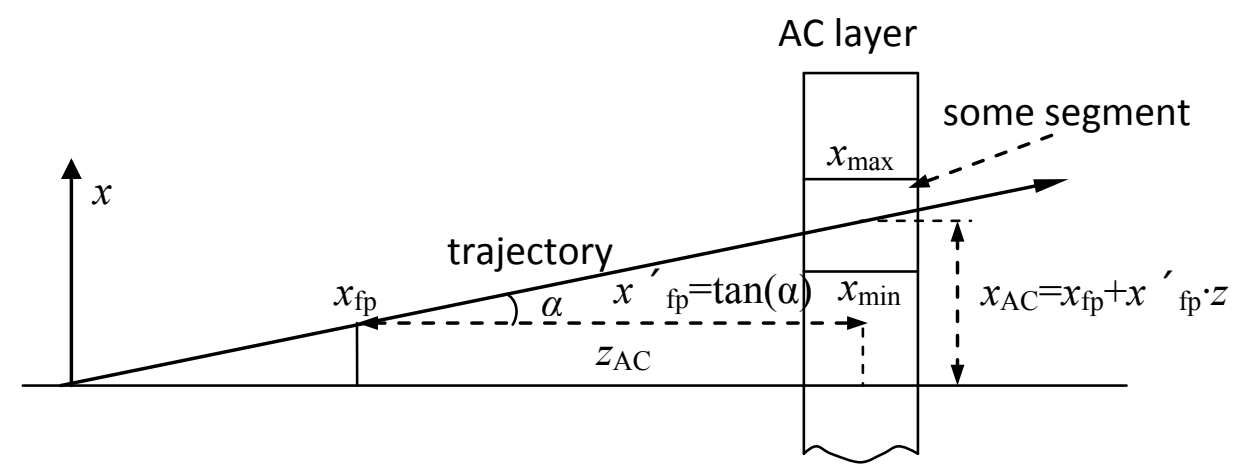

Figure 4.2.10 Projection of the particle track from focal plane on AC layer.

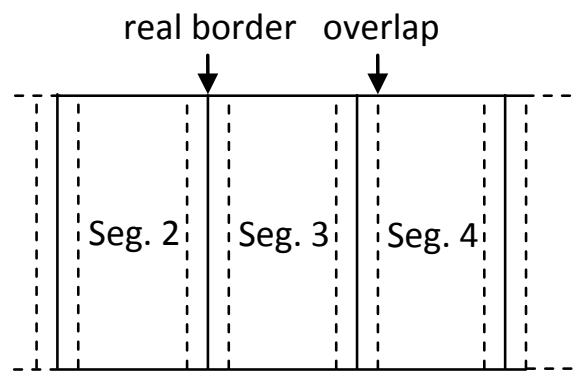

Figure 4.2.11 Geometrical overlap of the AC segments specified in the analysis software.

If the track points to the overlap region (the area between the close dashed lines in the Fig. 4.2.11) the code essentially assumes that it is equally likely that either segment has been hit by the true trajectory. It then gives the corresponding signals equal weight, independent of how far the track points from the border between the segments.

That such an equal treatment is not appropriate, which is demonstrated in the following two figures. The spectrum in Fig. 4.2.12 shows the projected track position for events that fire a particular segment and at the same time did not fire either of the 
neighboring segments. The events were selected by placing cuts on the corresponding TDC distributions:

$$
\left|t_{i-1}-\bar{t}_{i-1}\right|>\tau \&\left|t_{i}-\bar{t}_{i}\right|<\tau \&\left|t_{i+1}-\bar{t}_{i+1}\right|>\tau
$$

Here $i$ is the number of segment of interest, $\overline{t_{i}}$ the mean value of the good timing distribution, and $\tau$ the half width of the distribution.

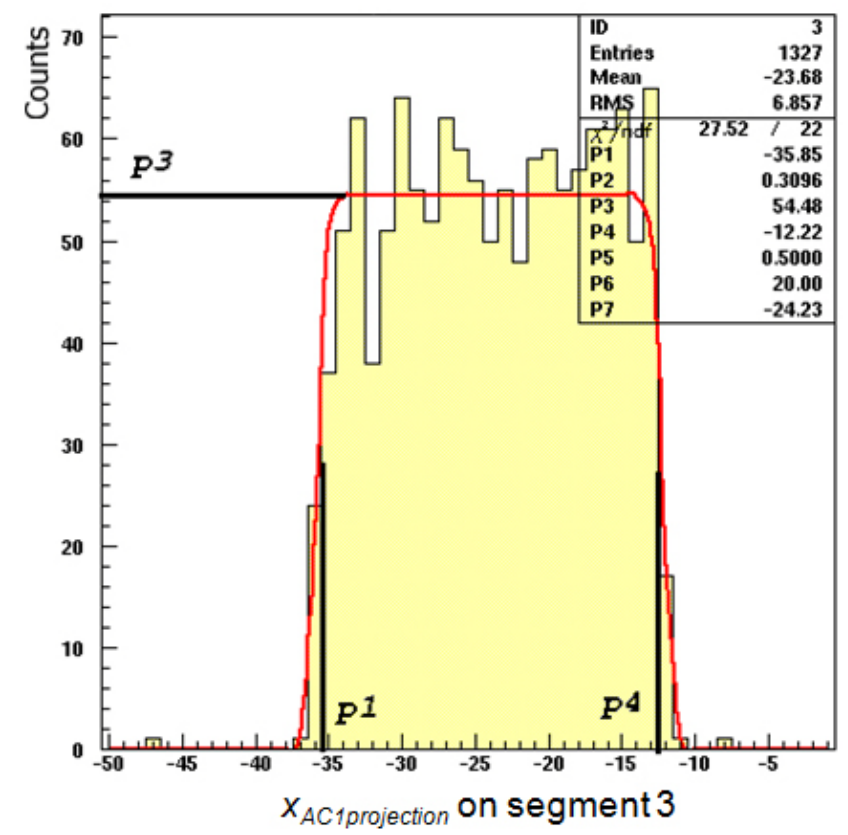

Figure 4.2.12 The $x$ projection on AC layer 1 segment 3. (Units are in $\mathrm{cm}$ ).

The width of the distribution is approximately of $23.6 \mathrm{~cm}$, which, as expected, is comparable with the segment's width, $23 \mathrm{~cm}$. This distribution was parameterized as a step function with smeared sides:

$$
x_{A C_{L(k)}}=\left\{\begin{array}{l}
p_{3} \cdot\left(1-1 /\left(1+\mathrm{e}^{\left(x-p_{1}\right) / p_{2}}\right)\right), x<\left(p_{4}+p_{1}\right) / 2, \\
p_{3} \cdot\left(1-1 /\left(1+\mathrm{e}^{\left(-x+p_{4}\right) / p_{5}}\right)\right), x>\left(p_{4}+p_{1}\right) / 2 .
\end{array}\right.
$$


Here $p_{3}$ is the height of the plateau of the distribution, $p_{1}$ and $p_{4}$ are the start and end of the step function, and $p_{2}$ and $p_{5}$ describe the left and right shoulders. These parameters, together with the fit, are shown in Fig. 4.2.12. The normalized fit results are stored in the likelihood PID parameter file.

Now, the AC probability for a trajectory, for example to be a kaon, is the probability given by segment, $A C_{i}^{k}$, multiplied with the probability of the segment being hit, $x_{i}$, summed over all segments in the layer:

$$
A C_{L}=\sum_{k=1}^{7} x_{A C_{L(k)}} \cdot A C_{L(k)}, \quad(L=1,2,3)
$$

When the particle traverses two segments at the same time, as it was schematically displayed in Fig. 4.2.9, we will obtain two $x$ projection distributions (Fig. 4.2.13). If we assume that we have an event in AC layer 1 somewhere between $x=-12$ and $x=-10$ $\mathrm{cm}$, as shown on the figure, we will read out two values for the $x$ projection pdf: $x_{p d f 3}$ and $x_{p d f 4}$. In such a case, the pdf for $\mathrm{AC}_{1}$ according to expression 4.2 .13 will be:

$$
A C_{1}=x_{p d f 3} \cdot A C_{1(3)}+x_{p d f 4} \cdot A C_{1(4)}
$$

The rest of the $x_{p d f}$ values for such an event are almost equal to zero and therefore do not contribute to $\mathrm{AC}_{1}$ pdf. In total we can form seven projection distributions for each $\mathrm{AC}$ layer, schematically shown in Fig. 4.2.14. Equation (4.2.14) is just an example of what would happen in the case of the specific particle trajectory described above. In the likelihood PID routine, embedded in the engine, we apply the general form described by Equation (4.2.13). 


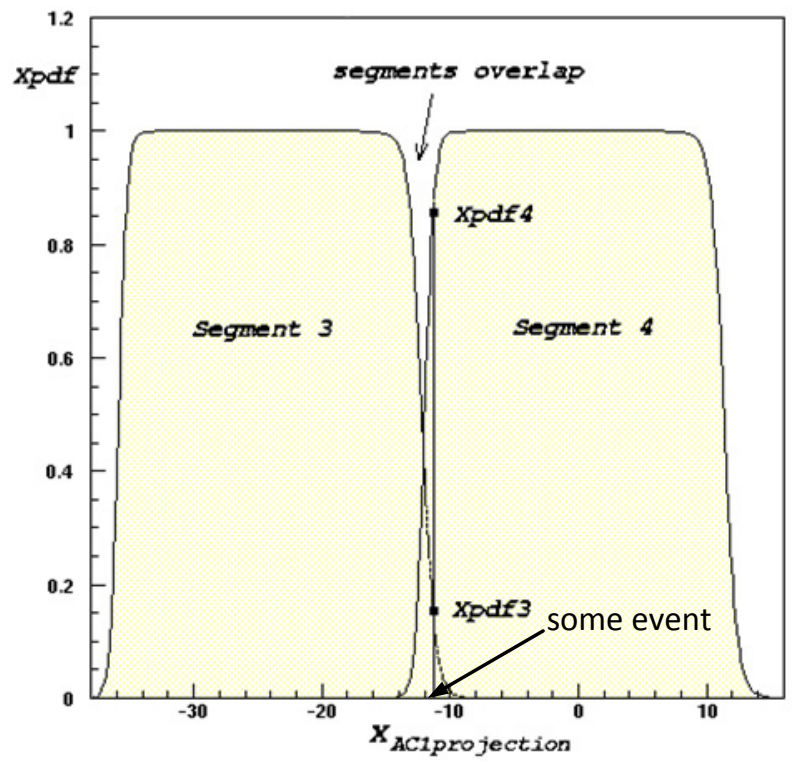

Figure 4.2.13 Example of the two neighbor segment fired at the same time.

\begin{tabular}{|l|l|l|l|l}
\hline 1 & 2 & 3 & 4 & $x_{A C_{L}}$ \\
\hline
\end{tabular}

Figure 4.2.14 Schematics of the $x_{A C_{L}}$ projections on segments 1,2,3, and etc.

\section{Water Cherenkov}

To compose probability density functions the water Cherenkov detectors we utilize the summed n.p.e. values of the $\mathrm{WC}$ layers: $\mathrm{WC}_{1}$ and $\mathrm{WC}_{2}$. The Cherenkov radiation created in water has a strong momentum dependence for protons and kaons (see Fig. 3.2.1). The pions' mean n.p.e. are virtually flat with momentum change. That said, $\mathrm{WC}_{1}$ and $\mathrm{WC}_{2}$ 
spectra must be obtained and parameterized for different particle momenta that lie within the spectrometer momentum acceptance of $1.2 \pm 0.15 \mathrm{GeV} / \mathrm{c}$. In order to do that, we applied $\pm 3 \%$ momentum cuts and obtained WC distributions for each momentum slice. An example for the proton, pion, and kaon $\mathrm{WC}_{1}$ and $\mathrm{WC}_{2}$ spectra for a slice with central momentum $p=1.25 \mathrm{GeV} / \mathrm{c}$ is presented in Fig. 4.2.15.
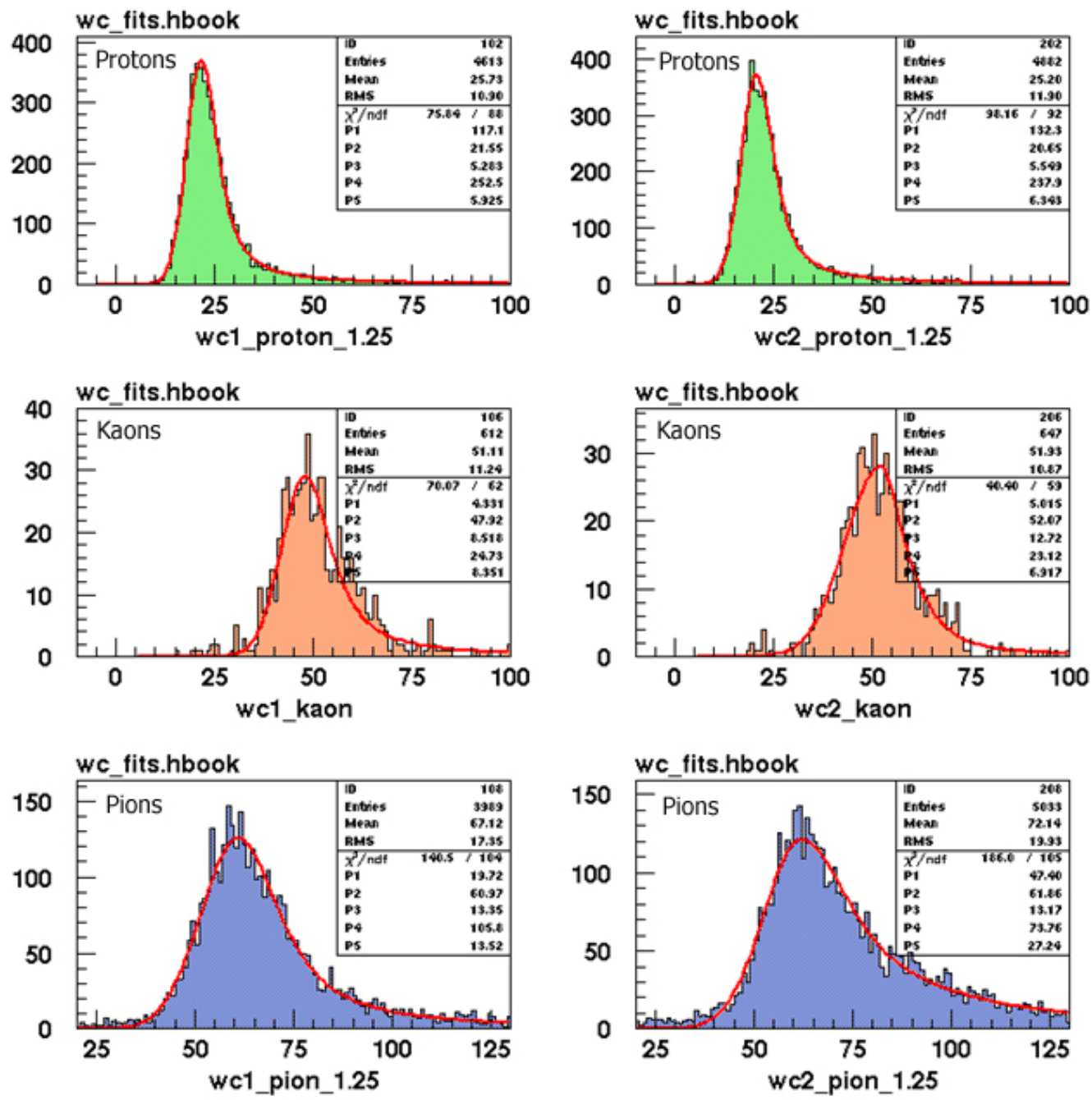

Figure 4.2.15 $\mathrm{WC}_{1}$ and $\mathrm{WC}_{2}$ proton, kaon and pion spectra for $\pm 3 \%$ momentum cut around $1.25 \mathrm{GeV} / \mathrm{c}$. 
Since all the histograms are characterized by a long tail on the right side, the Right Voigt function, Equation (4.2.9), has been used to fit distributions for all three particles. The fits were applied to proton and pion spectra for six momentum slices $p=$ $1.15,1.20,1.25,1.30,1.35,1.40 \mathrm{GeV} / \mathrm{c}$. It allowed us to have six distinct parameterizations separately for protons and pions (Fig. 4.2.16).
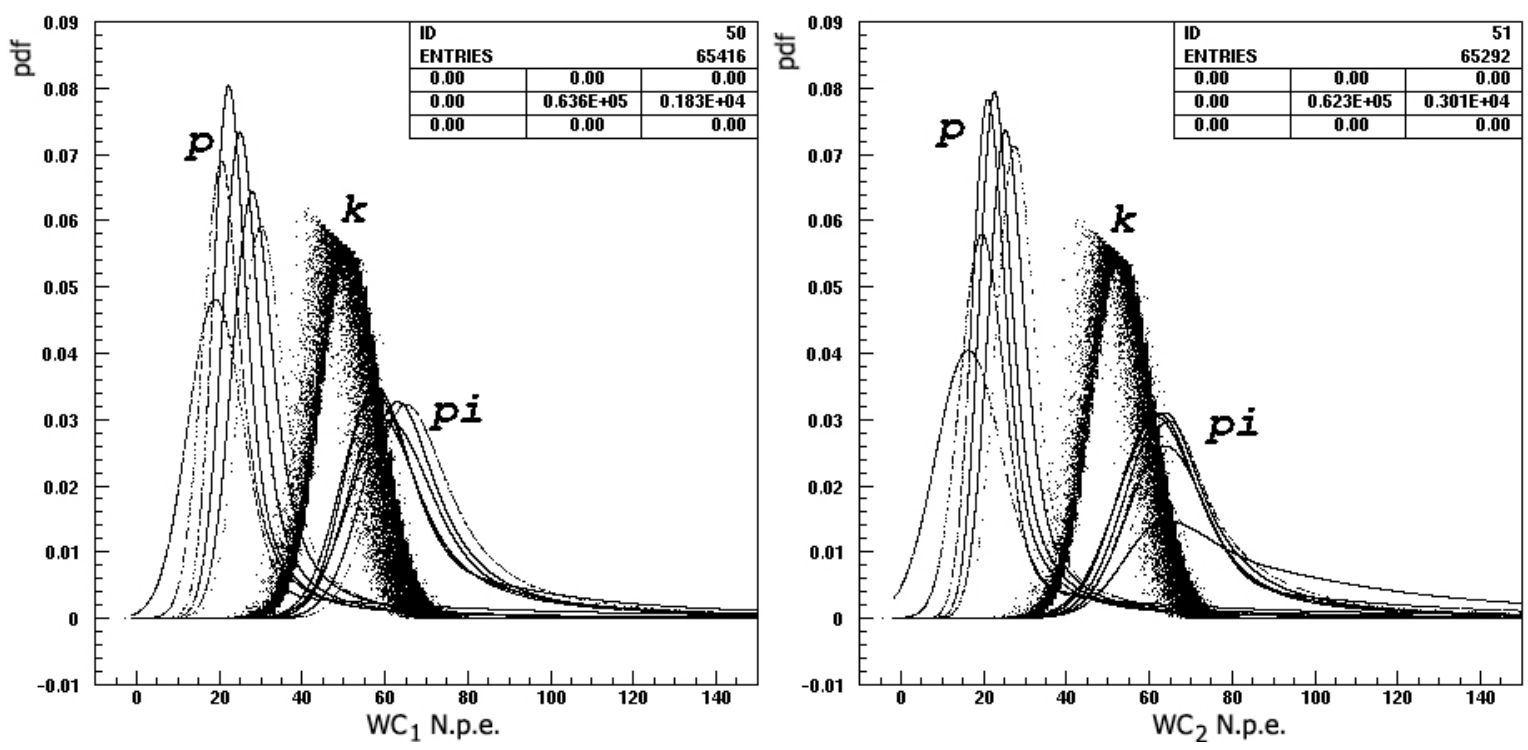

Figure 4.2.16 The $\mathrm{WC}_{1}$ and $\mathrm{WC}_{2}$ normalized pdf distributions for protons, kaons and pions. Protons and pions use six pdfs to account for momentum dependence. Kaons use Poisson function.

A similar momentum dependence study for kaons is quite a challenging task due to the relatively low statistics of kaons compared to protons and pions. We have selected a different approach to describe the kaons' pdfs. We used Equation (3.2.2) to predict the location of the kaon peak and further simulate it with the Poisson function. To do such an analysis, we found mean values for protons, kaons, and pions by fitting the corresponding n.p.e. distributions at different momenta. The momentum values were converted to 
$\sin ^{2}(\theta)$ and the graph of n.p.e. versus $\sin ^{2}(\theta)$ was plotted. An example of such data is shown in the Table 4.2.3. The plots from the table's data are presented on the Fig. 4.2.17. The absolute uncertainties for $\sin ^{2}(\theta)$ were found by error propagation of the spectrometer's momentum resolution $\Delta p / p=2 \cdot 10^{-4}$ and the absolute uncertainty of particle's mass available in the Particle Data Book [3]. The absolute errors for WC n.p.e. values were extracted from the fits. Since Equation (3.2.2) suggests a linear dependence, we applied a linear fit,

$$
\text { n.p.e. }=N_{0} \sin ^{2}(\theta)+C_{0} \text {, }
$$

to these data. The $C_{0}$ constant was introduced to account for background present in the n.p.e. spectrum. Such background is thought to be mostly created by the plastic walls of the WC diffusion container. The other possible source is the Amino-G-salt acid wavelength shifter. Now, knowing the momentum, we can estimate the kaon mean value $\mu_{\mathrm{K}}$ from expression (4.2.16). As soon as we have it calculated we use Poisson distribution,

$$
p_{K}=\frac{\mu^{x} e^{-\mu}}{x !} ; \quad(x \equiv n . p . e .),
$$

to simulate the kaon peak. The $\mathrm{WC}_{1}$ and $\mathrm{WC}_{2}$ kaon distributions can be seen in Fig. 4.2.16. Since the $\mu_{\mathrm{K}}$ is momentum dependent, there are many kaon distributions generated, as we can see on the figure. 
Table 4.2.3 WC parameterization for n.p.e. versus $\sin ^{2}(\theta)$ dependence.

\begin{tabular}{lccccccc}
\hline Particle & $\begin{array}{c}p \\
(\mathrm{GeV} / \mathrm{c})\end{array}$ & $\left(\sin ^{2} \theta\right) \times 10^{-1}$ & $\Delta\left(\sin ^{2} \theta\right) \times 10^{-}$ & $\mathrm{WC}_{1}$ & $\Delta(\mathrm{WC} 1)$ & $\begin{array}{c}\mathrm{WC}_{2} \\
\text { n.p.e. }\end{array}$ & $\Delta(\mathrm{WC} 2)$ \\
\hline Proton & 1.15 & 0.6257 & \pm 0.250 & 18.93 & \pm 0.050 & 16.19 & \pm 0.050 \\
$m=0.9383 \mathrm{GeV}$ & 1.2 & 0.9314 & \pm 0.373 & 20.57 & \pm 0.521 & 19.48 & \pm 0.245 \\
& 1.25 & 1.2012 & \pm 0.481 & 22.22 & \pm 0.127 & 20.91 & \pm 0.126 \\
& 1.3 & 1.4404 & \pm 0.576 & 24.98 & \pm 0.091 & 22.57 & \pm 0.080 \\
& 1.35 & 1.6352 & \pm 0.654 & 27.92 & \pm 0.110 & 25.21 & \pm 0.139 \\
& 1.4 & 1.8442 & \pm 0.738 & 30.04 & \pm 0.287 & 27.42 & \pm 0.191 \\
\hline Kaon & 1.25 & 3.4943 & \pm 1.416 & 49.56 & \pm 0.867 & 51.08 & \pm 1.336 \\
$m=0.4937 \mathrm{GeV}$ & & & & & & & \\
\hline Pion & 1.2 & 4.2961 & \pm 1.719 & 57.45 & \pm 0.617 & 63.75 & \pm 0.574 \\
$m=0.13957 \mathrm{GeV}$ & 1.25 & 4.3020 & \pm 1.721 & 57.49 & \pm 0.552 & 62.68 & \pm 0.487 \\
& 1.3 & 4.3073 & \pm 1.723 & 60.73 & \pm 0.424 & 61.48 & \pm 0.613 \\
& 1.35 & 4.3120 & \pm 1.725 & 63.05 & \pm 0.585 & 63.73 & \pm 0.495 \\
\hline
\end{tabular}
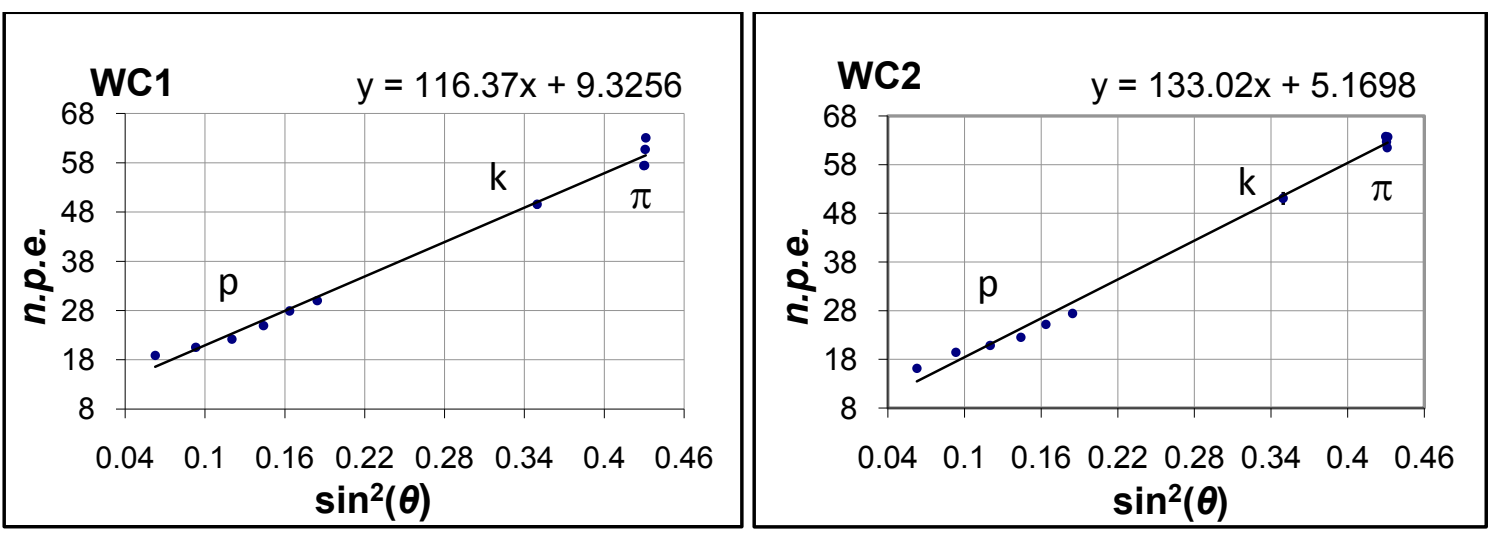

Figure 4.2.17 $\mathrm{WC}_{1}$ and $\mathrm{WC}_{2}$ n.p.e. dependence on squared sine of Cherenkov radiation angle.

\section{Beta and relative beta}

The relative velocity, beta, has the following momentum dependence:

$$
\beta=\frac{p}{E}=\sqrt{\frac{p^{2}}{p^{2}+m^{2}}} .
$$


In our analysis, we use the relative betas, $\beta_{i}^{\prime}=\beta-\beta_{i}$, to eliminate the momentum dependence. The $i$ in the formula means proton, kaon, and pion, and $\beta_{i}$ is calculated according to Equation (4.2.18) using the reconstructed momentum $p$ and the assumed particle mass $m$.
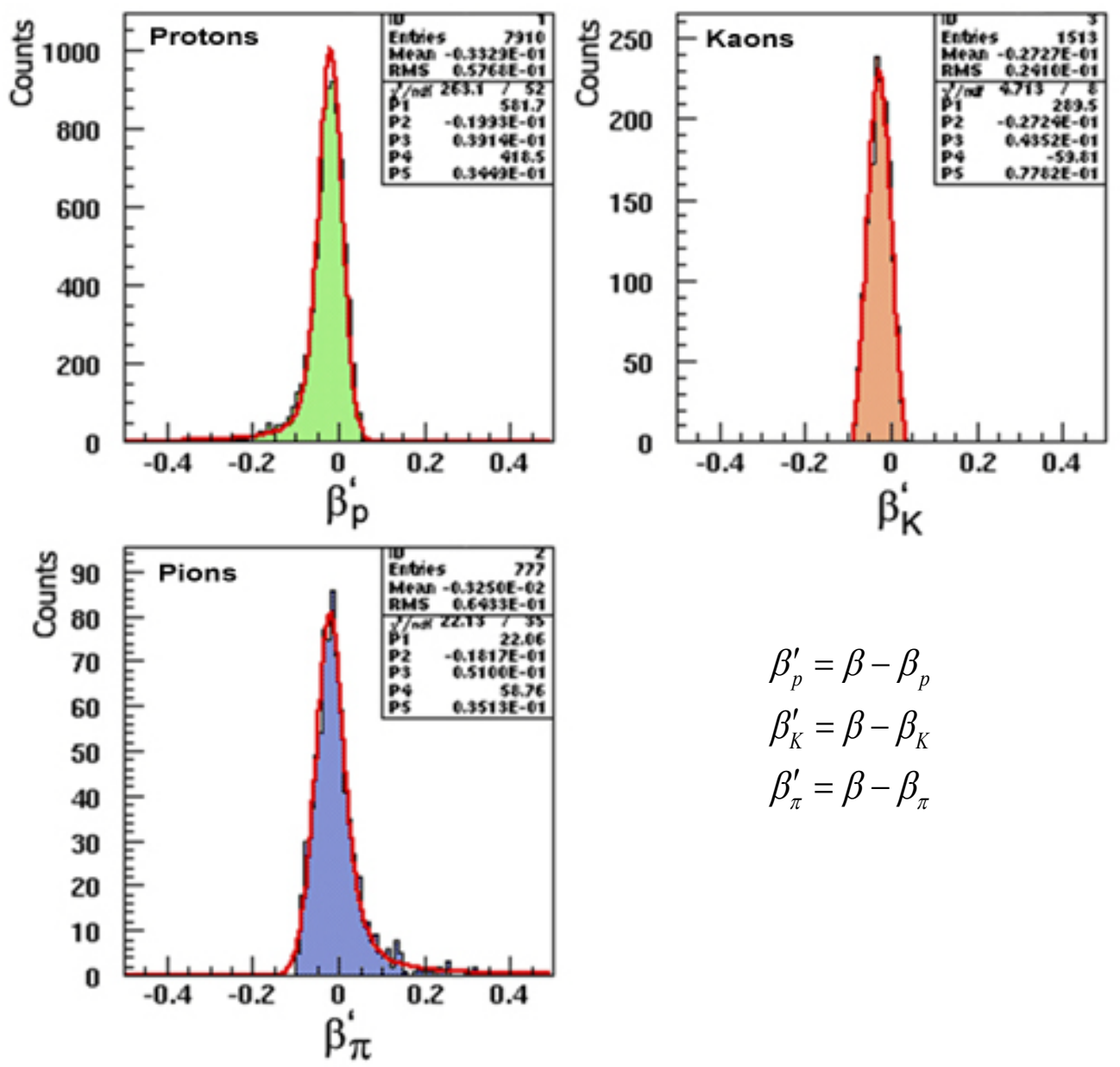

$$
\begin{aligned}
& \beta_{p}^{\prime}=\beta-\beta_{p} \\
& \beta_{K}^{\prime}=\beta-\beta_{K} \\
& \beta_{\pi}^{\prime}=\beta-\beta_{\pi}
\end{aligned}
$$

Figure 4.2.18 Relative beta "clean" distributions. 
The "clean" distributions for relative betas are shown in Fig. 4.2.18. Here for the protons, kaons, and pions we are using, respectively, Left Voigt, Central Voigt, and Right Voigt functions for fitting. The fit parameters are recorded in the likelihood PID parameter file and the normalized relative beta pdfs are later used in the analysis routine. Figure 4.2.19 shows how the momentum dependence of $p$ results in smeared distributions.

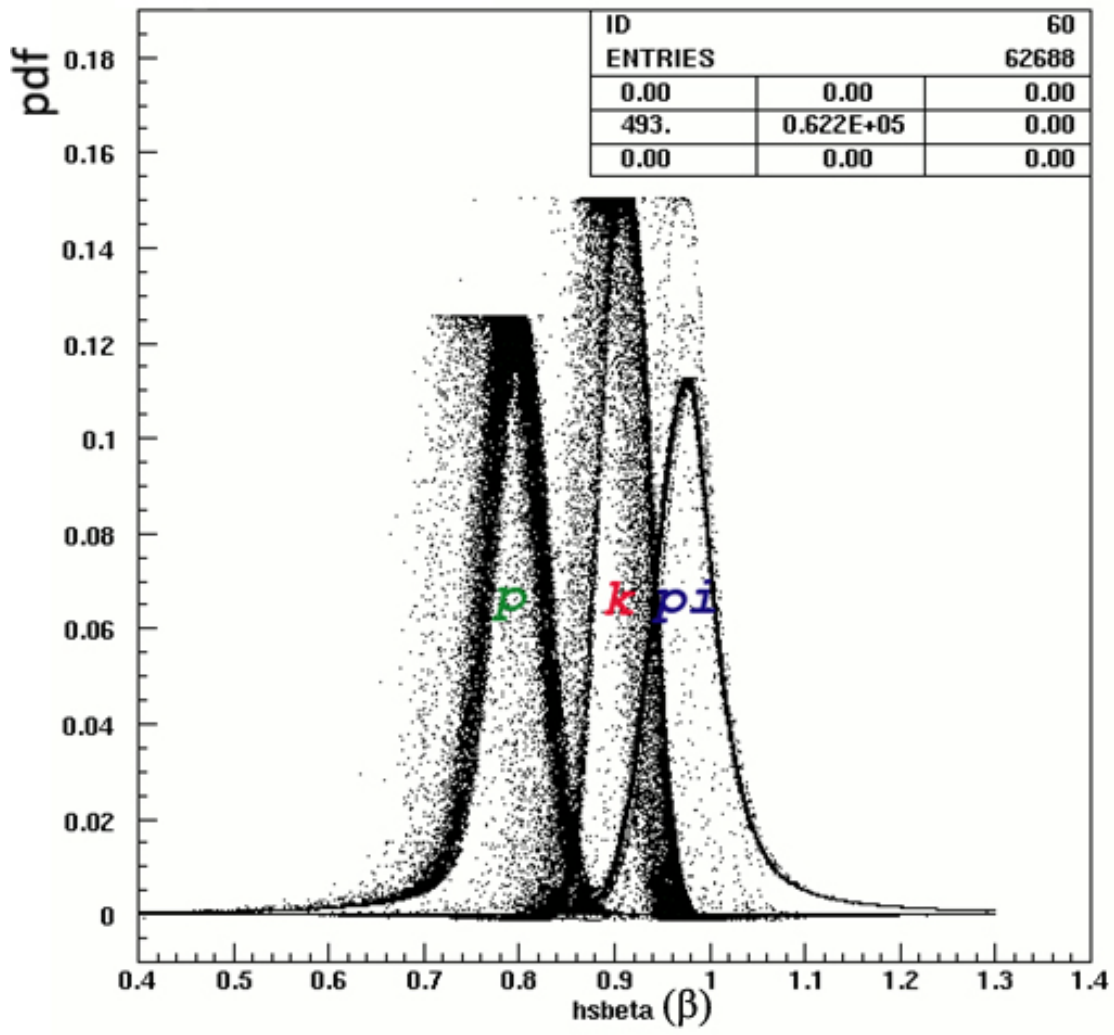

Figure 4.2.19 Normalized "clean" relative beta distributions on beta scale.

Having obtained probability density functions for $\mathrm{AC}$ and $\mathrm{WC}$ counters and relative betas one can now compose the final likelihood values. However, we will introduce one more step in the PID analysis that will account for the difference in the 
contributions of each of the three particles to the final data. We know that we have a huge number of protons and pions due to background reactions while the number of kaons is relatively small. In percentage we obtain $45.1 \%$ protons, $7.2 \%$ kaons, and $47.7 \%$ pions in the recorded data with the kaon PRE-triggered electronic settings. These numbers can vary depending on the target and trigger used. To introduce such particle fractions we have to look at the beta distribution and figure out the numbers of each particle contributing to the total spectrum. Such an estimate has been done with the application of a TripleVoigt fit to the beta spectrum (see Figure 4.2.20)

$$
\text { TripleVoigt }=\text { LeftVoigt }_{p}+\text { CentralVoigt }_{K}+\text { RightVoigt }_{\pi} \text {. }
$$

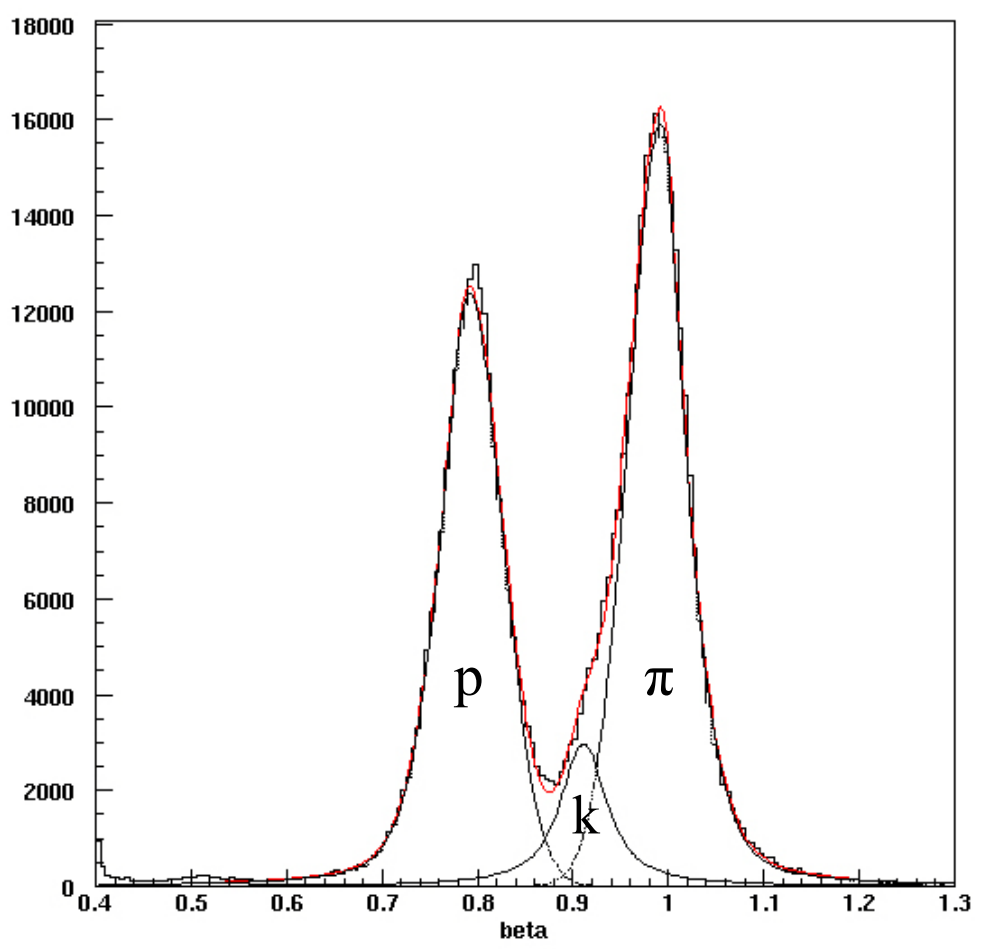

Figure 4.2.20 TripleVoigt fit of the beta spectrum, with protons described by LeftVoigt, kaons - by CenralVoigt and pions - by RightVoigt functions. 
The peaks' mean values were extracted from the "clean" beta distributions and further were fixed in the TripleVoigt fit, while the width and strength were kept free. The ratios of the peaks' integrals to the total number of events in the spectrum provided the information about the fractions. In such way

$$
f r a c_{p}+f r a c_{K}+f r a c_{\pi}=1
$$

\subsection{Likelihood values composition}

All of the PID pdf variables: AC, WC, relative beta, and fractions extracted from the previous section behave as statistically independent units. Following the multiplication rule for independent events we compose the final likelihood values

$$
L_{i}=\frac{\prod_{k=1}^{7} p d f_{k}^{i}}{\sum_{i=1}^{3} \prod_{k=1}^{7} p d f_{k}^{i}}=\frac{A C_{1}^{i} \cdot A C_{2}^{i} \cdot A C_{3}^{i} \cdot W C_{1}^{i} \cdot W C_{2}^{i} \cdot \beta_{i}^{\prime} \cdot f r a c_{i}}{\sum_{i=1}^{3} A C_{1}^{i} \cdot A C_{2}^{i} \cdot A C_{3}^{i} \cdot W C_{1}^{i} \cdot W C_{2}^{i} \cdot \beta_{i}^{\prime} \cdot f r a c_{i}}
$$

where $i$ defines the particle as: $i=1$ (protons), 2(kaons), 3(pions). All three likelihood values calculated in such way are normalized and, therefore, add up to one: $L_{p}+L_{K}+L_{\pi}=1$. As it was mentioned before, the estimate of the likelihood values is performed inside of the analyzer's engine. Further, conditions have to be applied to these likelihood values to differentiate between the particles. The simplest condition used for kaon selection is

$$
\left(L_{K}>L_{p}\right) \&\left(L_{K}>L_{\pi}\right)
$$


This condition is embedded in the data reduction routine (see Figure 4.2.6), which skims the analyzed data to leave only kaons. The example of the AC, WC, and beta spectra after application of the likelihood PID, based on condition (4.2.22), is shown in Fig. 4.2.21. The location of the particles' peaks in all distributions matches the expectation. Now the condition (4.2.22) can be used as an alternative to (4.2.6), where the limiting cuts on all distributions are applied.

\subsubsection{Comparison of standard and likelihood PID methods}

Since the likelihood method employs only pdfs to make particle selection in contrast to limiting cuts used in standard approach, we expect to obtain a higher kaon yield. To test this we compare two data sets with different PID methods but the same cuts:

$$
\begin{gathered}
\left|\delta_{H K S}\right| \leq 20 \% \&\left|\delta_{E N G E}\right| \leq 60 \% \\
\left|t_{H K S_{f p}}-20\right| \leq 30 \&\left|\chi_{H K S}^{2}\right| \leq 50, \\
\left|x_{H K S_{t a r}}^{\prime}-0.006\right| \leq 0.4 \&\left|y_{H K S_{t a r}}^{\prime}+0.006\right| \leq 0.1 .
\end{gathered}
$$

The first two cuts, (4.2.23), use the relative momenta that are defined as: $\delta_{H K S}=\left(p^{H K S}-p_{0}^{H K S}\right) / p_{0}^{H K S}$ for HKS and $\delta_{E N G E}=\left(p^{E N G E}-p_{0}^{E N G E}\right) / p_{0}^{E N G E}$ for ENGE. The values of relative momenta are measured by the drift chambers. The other two cuts, (4.2.24) select only the HDC signal that has good timing in the HKS focal plane and belongs to a good track. The cuts (4.2.25) make sure that ENGE target variables are in the expected range. All cuts, (4.2.23), (4.2.24), and (4.2.25) are used in "AND".

The cut conditions that allow us to perform PID in different ways are: 
1) $\left(\mathrm{AC}_{1}+\mathrm{AC}_{2}+\mathrm{AC}_{3}\right)<6 \&\left(\mathrm{WC}_{1}+\mathrm{WC}_{2}\right)>75 \&\left|\beta-\beta_{K}\right|<0.06$ - for standard PID.

2) $\left(L_{K}>L_{p}\right) \&\left(L_{K}>L_{\pi}\right)$ - for likelihood approach.

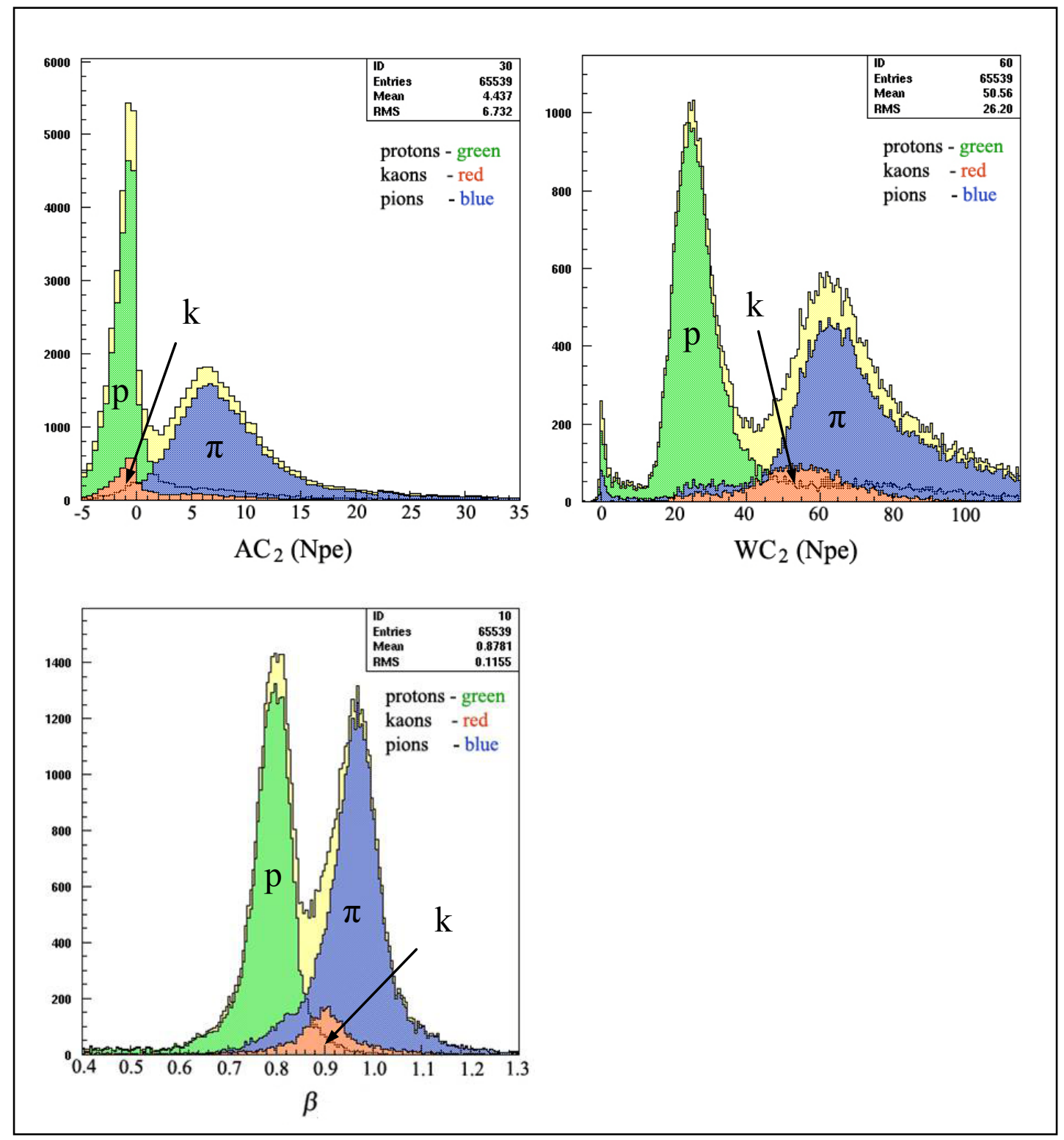

Figure 4.2.21 Example of AC, WC, and beta spectra obtained by likelihood PID. (The vertical axes are in Counts.) 


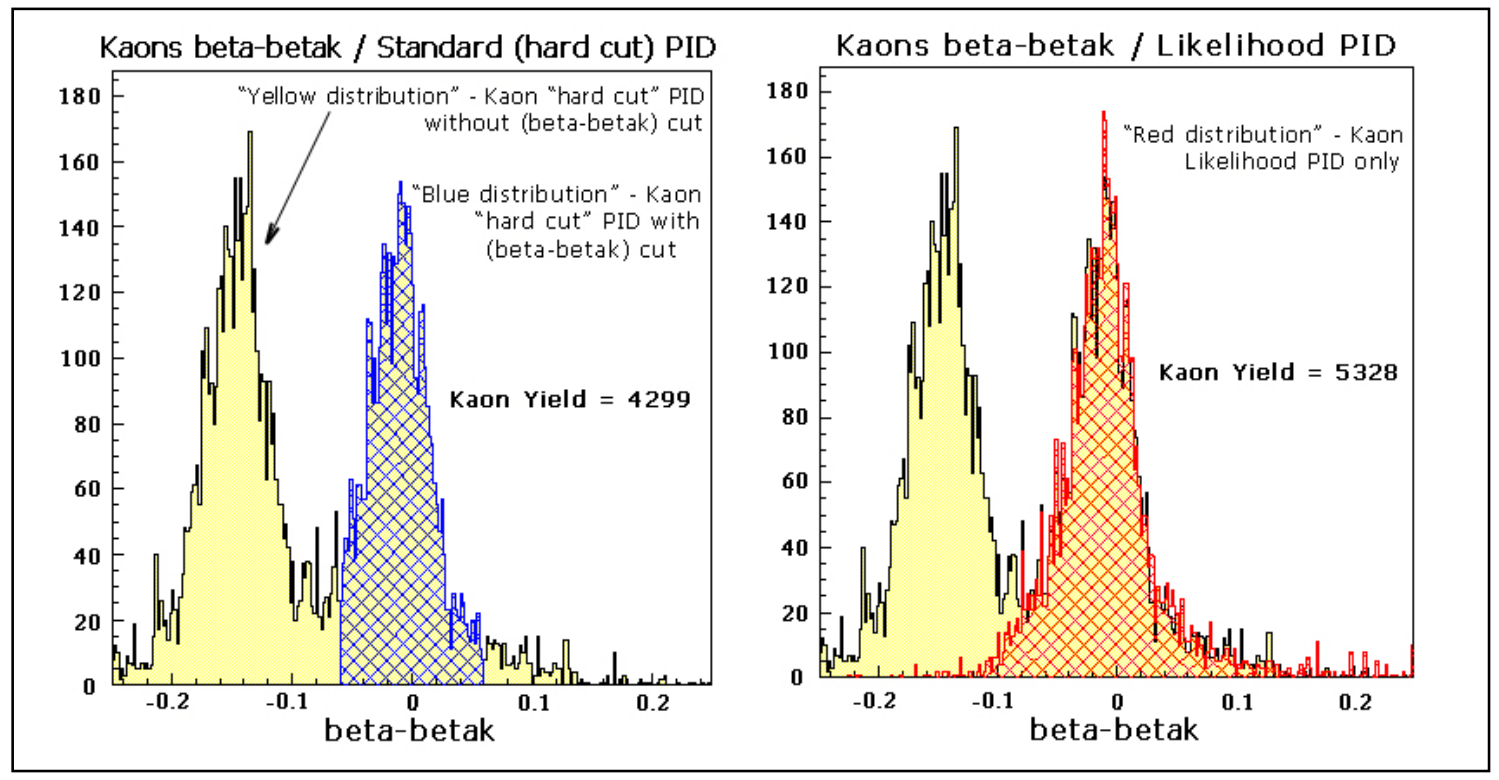

Figure 4.2.22 The spectrum of relative kaon beta, $\beta$ - $\beta_{K}$, for standard hard-cut PID on the left and likelihood on the right. (The vertical axes are in Counts).

Figure 4.2.22 shows a side-by-side comparison of relative kaon beta, $\beta-\beta_{K}$, obtained by the discussed methods. The blue colored distribution on the left is the result of the standard PID technique and the red colored histogram shows events extracted by the likelihood approach. The yellow distribution, shown behind each of the peaks, is obtained by applying only acceptance cuts and standard PID cuts, excluding the cut on the absolute value of relative beta variable, $\left|\beta-\beta_{K}\right|<0.06$. The first peak in the yellow distribution corresponds to protons and the second one belongs to kaons. As we can see, the application of only AC and WC variables in the hard cut PID technique cannot completely separate protons from kaons. For the kaons chosen by the limiting beta cut, (blue histogram), the right tail from proton distribution is present in kaon selection as a contamination. Furthermore, the application of limiting beta cut removes the kaons, which belong to the tails of the true kaon distribution. This is not the case for the 
likelihood approach, where no limiting cuts are applied except of the conditional cut on three likelihood variables. The likelihood-based PID results in a distribution that includes the tails and therefore yields a higher number of kaons than the standard PID. According to this comparison, a $19 \%$ gain in kaon yield is expected when applying the likelihood approach instead of the conventional (hard-cut) approach.

To explore further the PID improvement by the likelihood method, we compare the beta spectra obtained by likelihood and standard based PID inside of the $\left|\beta-\beta_{K}\right|<0.06$ range. The result of this comparison is shown in Fig. 4.2.23.

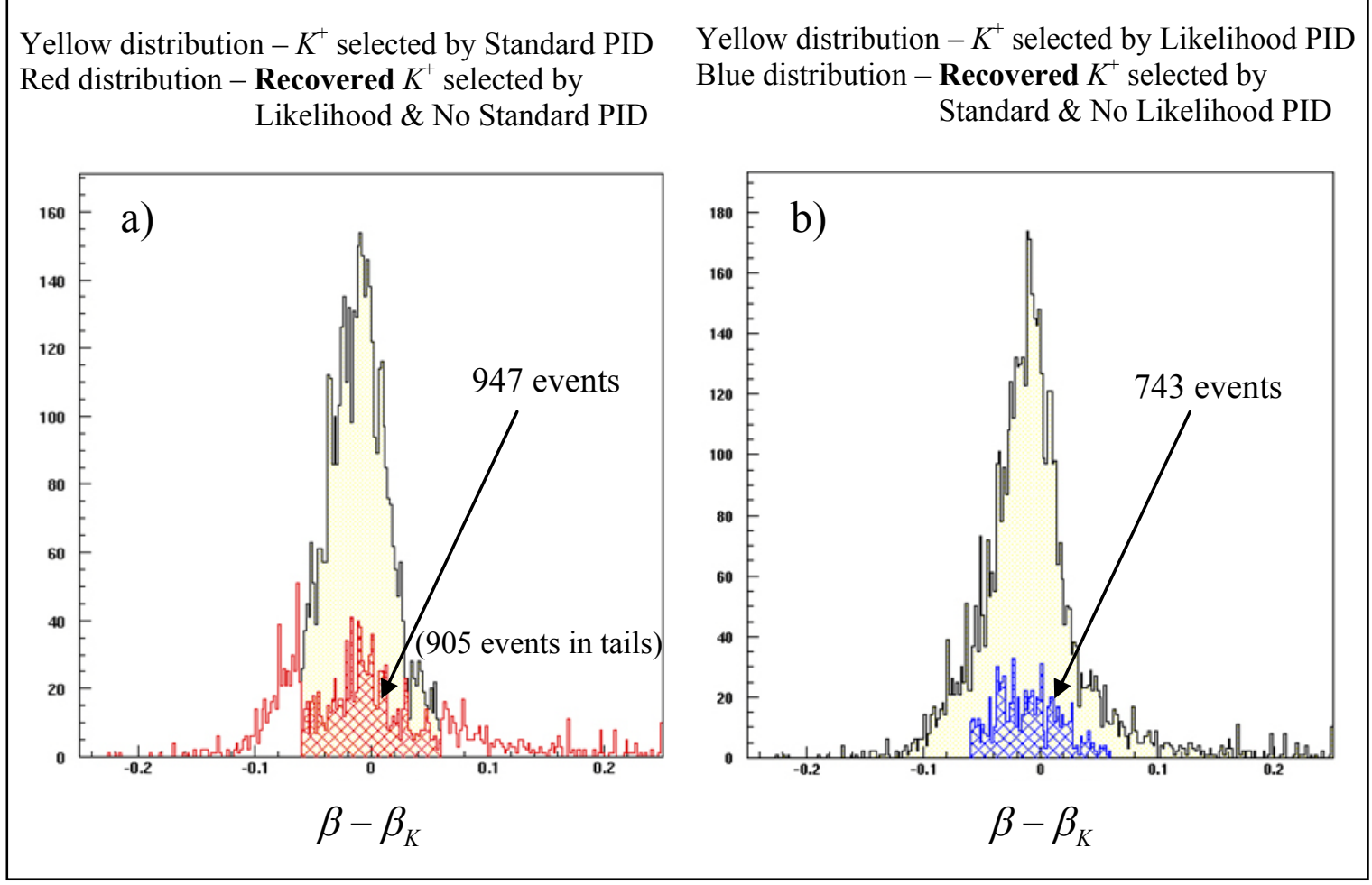

Figure 4.2.23 Comparison of the kaons recovered by: a) Likelihood.AND.NOT.(Standard) kaon PID; b) Standard.AND.NOT.(Likelihood) kaon PID. 
In plot $a$, the $\beta-\beta_{K}$ spectrum is generated by kaon likelihood PID in AND with NOT(Standard) kaon PID. By that we find kaon events that are recovered by the likelihood approach, and are not identified by the hard-cut technique. In total $947+905=1852$ kaons are obtained, where 947 events are located inside of the limiting $\left|\beta-\beta_{K}\right|<0.06$ cut and the rest belong to tails. Now, if we make the contrary comparison, i.e., standard kaon PID in AND with NOT(Likelihood), we only recover 743 kaon events, plot $b$. Comparing the plots $a$ and $b$ we conclude that the likelihood approach has a potential of recovering more kaon events than the standard method. The $\left|\beta-\beta_{K}\right|<0.06$ limiting cut area already by itself recovers more kaons in the case of the likelihood PID. Moreover, the kaon events present in the tails might significantly increase the kaon statistics in the final missing mass distribution. At this moment we have a good indication of the superiority of the likelihood method. However, the final comparison has to be made on the missing mass spectra, where we can confirm if the extra recovered kaon events contribute to the physics peak or background distribution.

\subsection{ENGE data analysis}

The data analysis procedure for the ENGE spectrometer is relatively simple compared to that for the HKS. The only negative particles with significant yield are recoil electrons. Therefore the ENGE analysis does not require any PID and only has to consist of tracking and reconstruction parts.

The position and angle at the focal plane is measured by the ENGE drift chamber (EDC) and timing information is extracted from two ENGE hodoscope layers, EHODO1 
and EHODO2. The EDC tracking routine works similarly to that of the HDC, however, the $\chi^{2}$ minimization routine consists of three loops. Initially the first track is composed without knowledge of time and only based on the wire hit pattern. Then the TDC information is included and a comparison with the initially guessed track is made. In the third loop, a $\chi^{2}$ minimization is applied to form the final track. The timing information of each scintillator is calibrated to account for propagation time in the paddle, time walk initiated by pulse high variation, and timing offsets due to cable length differences and electronics delays. These time correction procedures are identical to the HKS hodoscopes.

After the focal plane coordinates are found, the ENGE transformation matrix is applied to reconstruct the target variables. The ENGE reconstruction routine is similar to the HKS routine and calculates three ENGE variables $\delta, x_{t a r}^{\prime}$, and $y_{\text {tar }}^{\prime}$.

\subsection{Coincidence events}

To determine electron kaon coincident events, a variable called the coincidence time $t_{\text {coin }}$ is used. It is defined as:

$$
t_{\text {coin }}=t_{H K S . t a r}-t_{E N G E . t a r}-T D C_{H K S} \text {. }
$$

Here $t_{H K S . t a r}$ and $t_{E N G E . t a r}$ are the target time variables reconstructed from the focal plane single arm HKS and ENGE times. The $T D C_{H K S}$ is the coincidence TDC signal started by a kaon and stopped by the electron trigger. The single arm time at the focal plane was 
measured by the corresponding hodoscope planes. With the help of GEANT simulations, the path length for trajectories through the spectrometers was determined. Using this path length together with the focal plane time, the time of each event at the target was found. Properly calibrated coincidence time (cointime) has to contain the RF structure of the electron beam. The beam pulse rate was $499 \mathrm{MHz}$. Therefore, the 1/499.E6 = 2 (ns) periodic structure in the coincidence time serves as both a signature of the RF wave and an indicator of the proper calibration.

The coincidence time is a very important variable that would allow us to separate the true coincidence events from the accidental background events. To use this information reliably one needs to introduce some corrections to the coincident events. Typically the corrections are made to the coincidence time spectrum to account for blocked coincidences from a random prescaled event, self-timing events from a late trigger generated by one of the spectrometers, and loss of synchronization between the detectors and the spectrometers caused by operating the data acquisition system in a "buffered" mode. After all corrections are applied, the coincidence time is shown in Fig. 4.4.1. 


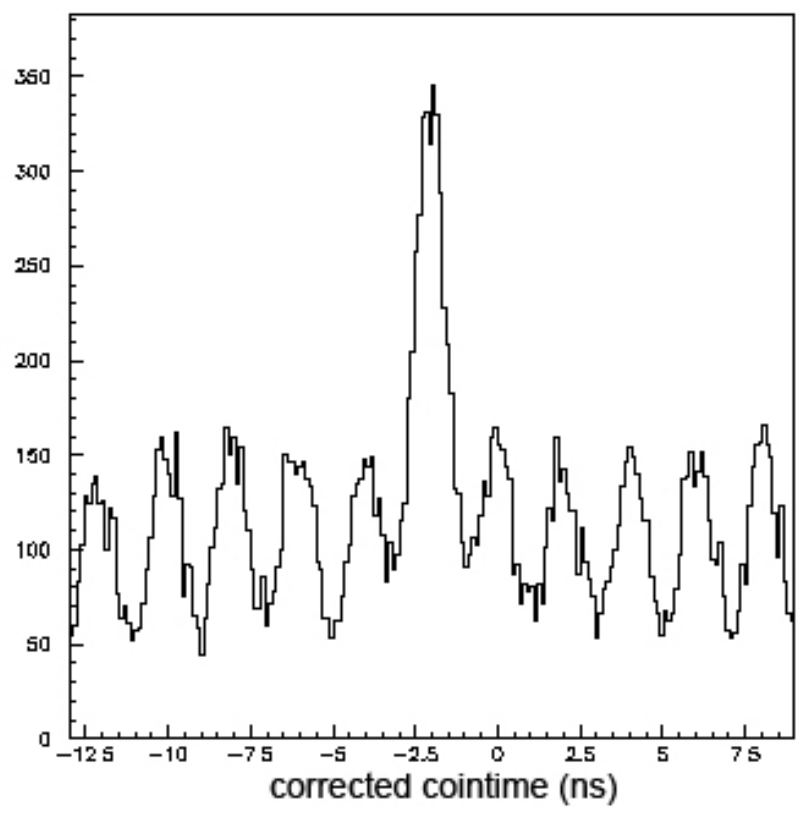

Figure 4.4.1 Coincidence time for all particle types after applied corrections.

As was expected, the spectrum has a periodic structure with 2 ns separation between peaks. The largest peak contains the true coincident events with an embedded accidental background. The two dimensional profile of relative velocity $\beta_{\text {TOF }}-\beta_{K}$ versus the coincident time, is shown in Fig. 4.4.2. The plot displays all three particles. The areas selected by rectangles correspond to the true coincident events with embedded accidentals for each of the three particles. 


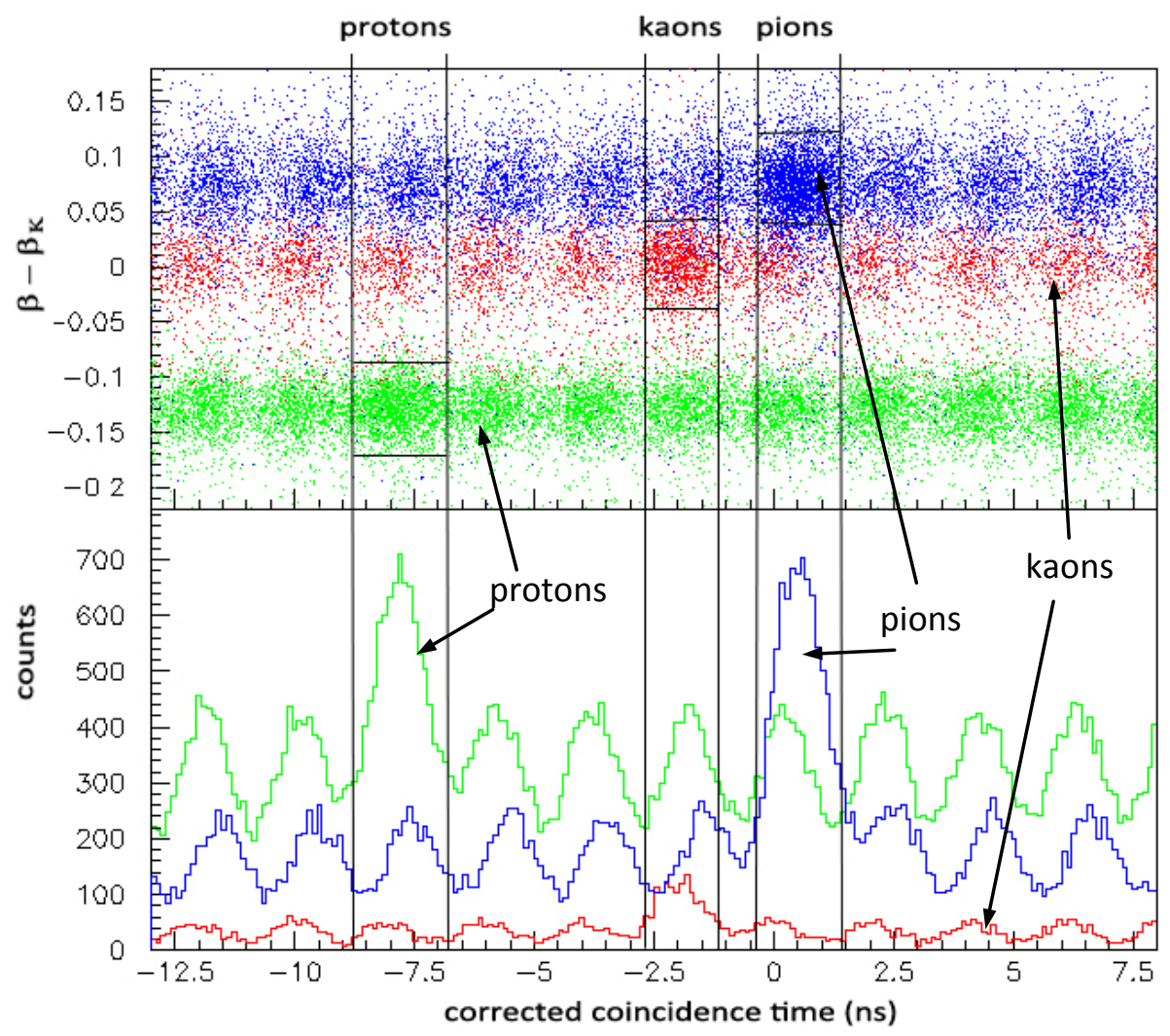

Figure 4.4.2 Two dimensional profile of $\beta_{T O F}-\beta_{K}$ versus coincidence time. The selected areas belong to true coincidence events with fraction of accidental events.

The procedure of separating the true from accidental coincidence events is straightforward. For this purpose we use the coincidence time spectrum with kaon PID. The largest peak is fitted with a Gaussian and a 2 ns hard cut, $\Delta t_{\text {coin.mean }}= \pm 1 \mathrm{ns,}$, is placed around its mean value. In such a way, the true coincidence events with some of accidentals are selected. These events correspond to a red hatched region in the center in Fig. 4.4.3.

Now, the accidentals present in the selected peak have to be subtracted. To do that, the number of accidentals inside of a selected region must be calculated. It is 
achieved by probing the accidental peaks as shown on the figure by the blue hatched area. The accidentals are then estimated as an integral of the selected peaks divided by the number of peaks, $A=\sum$ peaks $/ N_{\text {peaks }}$.

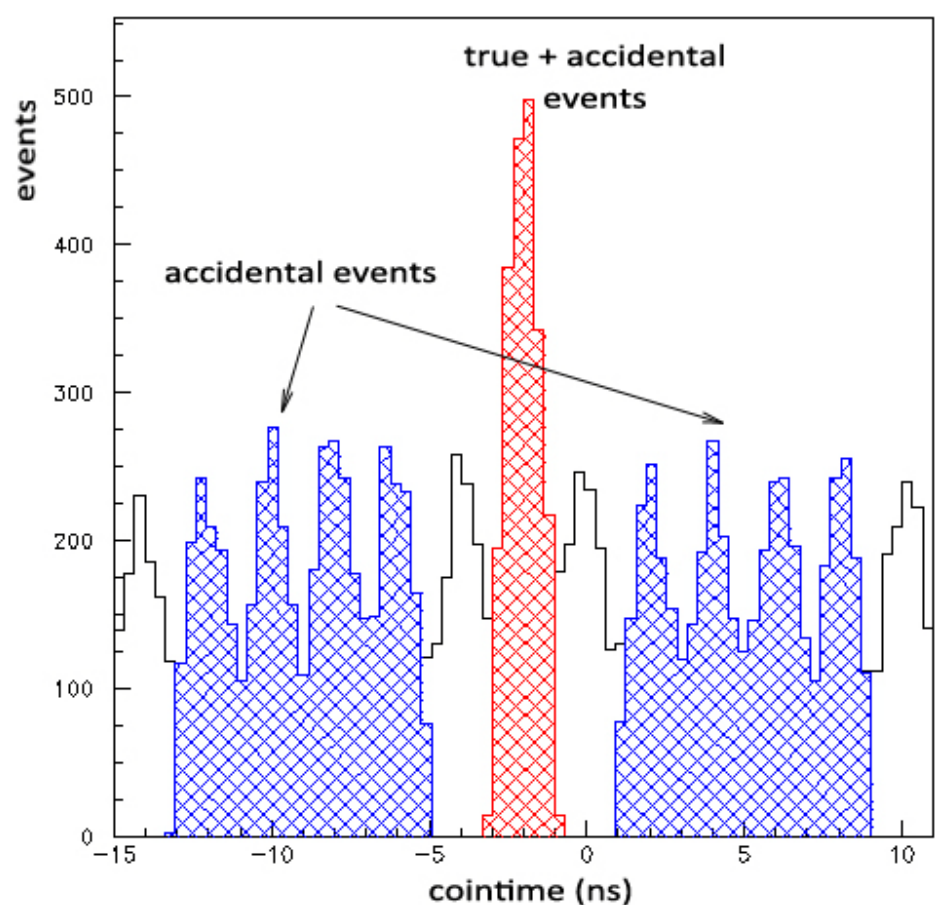

Figure 4.4.3 Coincidence time spectrum with true and accidental peaks separation technique.

\subsection{Missing mass spectrum}

As it was described in Section 5 of Chapter 1 the missing mass is calculated by expression (1.5.18), which uses the energy of hypernucleus (1.5.17) and its momentum (1.5.16). It is clear that in order to produce the missing mass spectrum we must know the hypernuclear energy, $E_{H}$, momentum, $p_{H}$, and the angles of the recoiled reaction products, $\theta_{e e^{\prime}}, \theta_{e K}$, and $\theta_{e^{\prime} K}$. After reconstruction process we have the angles $x_{t a r}^{\prime}, y_{t a r}^{\prime}$, and relative 
momentum $\delta$ calculated for both ENGE and HKS spectrometers. The relative momenta $\delta_{\mathrm{e}^{\prime}}$ and $\delta_{\mathrm{K}}$ provides us the recoil electron and kaon momenta. Further their energy are calculated as $E=\sqrt{p^{2}+m^{2}}$. The reconstructed angle tangents on the target are used to calculate the reaction angles,

$$
\begin{aligned}
& \cos \left(\theta_{e e^{\prime}}\right)=\frac{1}{\sqrt{\left(x_{\text {tar. } \text {.ngge }}^{\prime}\right)^{2}+\left(y_{\text {tar.enge }}^{\prime}\right)^{2}+1}}, \\
& \cos \left(\theta_{e K}\right)=\frac{1 \sqrt{ }}{\sqrt{\left(x_{t a r \cdot h k s}^{\prime}\right)^{2}+\left(y_{t a r h h s s}^{\prime}\right)^{2}+1}},
\end{aligned}
$$

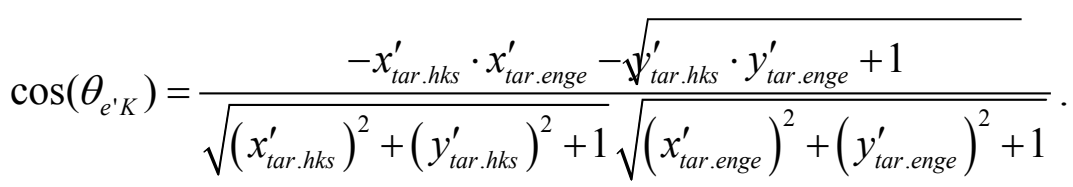

Equation (1.5.16) allows us to calculate the momentum and Equation (1.5.17) - the energy of the hypernucleus. Employing expression (1.5.18) we can calculate the hypernuclear missing mass.

\subsection{Momentum calibration}

The calibration procedure runs according to the following flowchart (Fig. 4.6.1).

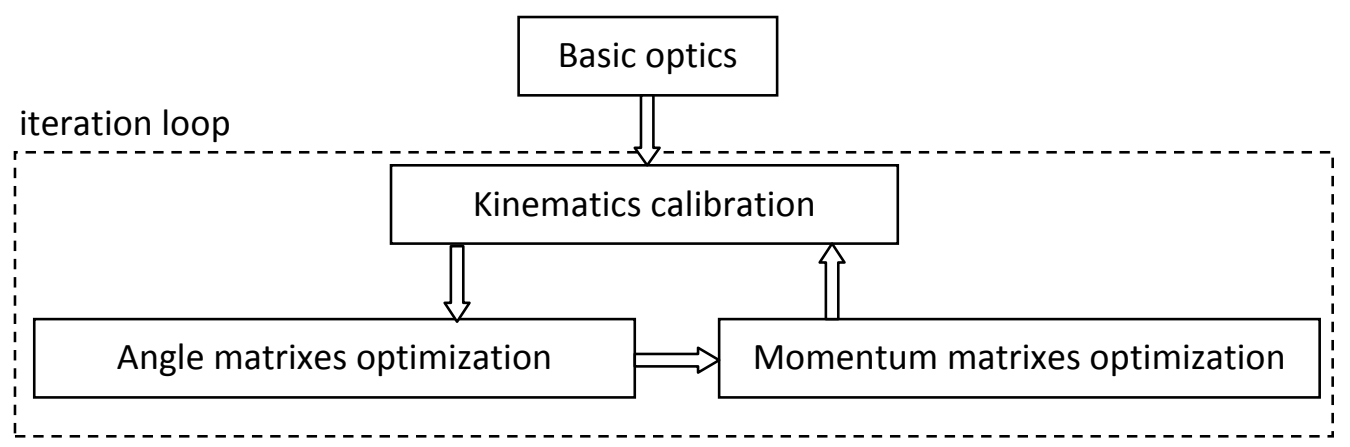

Figure 4.6.1 Flowchart of iteration process of optics calibration procedure. 
The basic optics calibrations are found by comparison of the optical simulation with data. Then the basic optics calibrations are fed into the kinematics calibration, which corrects for the beam energy and the electron and kaon central momenta. Further the sieve slit data is used for angle matrix optimization and the two arm momentum calibration is performed before folding back into the iteration loop.

\subsubsection{Kinematics calibration}

The kinematics calibration is performed for the purpose of finding the true value for the energy of the electron beam and the central momentum of both ENGE and HKS spectrometers. The kinematics scan searches for the energy and momentum offsets: $\Delta E_{b}$, $\Delta p_{K}^{0}$, and $\Delta p_{e^{\prime}}^{0}$. The two parameters, position and width, are subject to optimization. The procedure defines two $\chi^{2}$ values, one for each optimization parameter

$$
\begin{gathered}
\chi_{\text {pos }}^{2}=\sum_{i} w_{i}\left(M_{i}^{\text {calc }}-M_{i}^{P D B}\right)^{2}=w_{\Lambda}\left(M_{\Lambda}^{\text {calc }}-M_{\Lambda}^{P D B}\right)^{2}+w_{\Sigma}\left(M_{\Sigma}^{\text {calc }}-M_{\Sigma}^{P D B}\right)^{2}, \\
\chi_{\text {width }}^{2}=\sum_{i} w_{i} \sigma_{i}^{2}=w_{\Lambda} \sigma_{\Lambda}^{2}+w_{\Sigma} \sigma_{\Sigma}^{2},
\end{gathered}
$$

where $w_{\Lambda}$ and $w_{\Sigma}$ are the weight factors, and $\sigma_{\Lambda}$ and $\sigma_{\Sigma}$ are the missing mass resolutions for the $\Lambda$ and $\Sigma^{0}$ hyperons. The weight factors account for the yield difference between the $\Lambda$ and $\Sigma^{0}$ peaks. The optimal $w_{\Lambda}=2$ and $w_{\Sigma}=1$ have been chosen for calibration analysis. This 2 to 1 ratio was found from the relative error minimization analysis. 
In order to present the $\chi^{2}$ variance as a function of the kinematics, an additional kinematics variable was introduced

$$
V_{k i n}=\Delta E_{b}-\Delta p_{K}^{0}-\Delta p_{e^{\prime}}^{0}
$$

The calibration procedure searches the minimum of the $\chi_{\text {pos }}^{2}$ and $\chi^{2}$ width for different values of $V_{k i n}$. It runs in the loop until the minimum values are found and the kinematics offsets are defined. The detailed description of the optimization procedure can be found in $[75]$.

\subsubsection{Momentum calibration}

The HKS momentum calibration takes advantage of precise knowledge of masses of $\Lambda$ and $\Sigma^{0}$ hyperons together with the ground state of ${ }_{\Lambda}^{12} \mathrm{~B}$, available in the Particle Data Book [3]. To produce exclusive $\Lambda$ and $\Sigma^{0}$ hyperons we use the $5 \mathrm{~mm} \mathrm{CH}_{2}$ foil. The ground state binding energy of ${ }_{\Lambda}^{12} \mathrm{~B}$, previously found in emulsion experiment, allows adjusting the position of the peak's center and missing mass scale. The ${ }_{\Lambda}^{12} \mathrm{~B}$ ground state events are extracted from runs on ${ }^{12} \mathrm{C}$ target. The forward angle kinematics gives the missing mass as

$$
m_{H}^{2}=m_{A}^{2}-m_{K}^{2}-2 E_{K} m_{A}-2\left(E_{K}-p_{K} \cos \left(\theta_{K}\right)-m_{A}\right)\left(p_{e}-p_{e^{\prime}}\right)
$$


For the known hypernuclear masses, the momentum correlation between recoil electron and outgoing kaon is shown in Fig. 4.6.2.

The calibration is an iterative process. It starts from calculating the missing mass with the help of an existing reconstruction matrix and the initial, non-calibrated, momentum matrix. The $\chi^{2}$ for the produced mass is

$$
\chi^{2}=\sum_{i} w_{i}\left(M^{\text {calc }}-M^{P D B}\right)^{2} p_{i}^{\prime}
$$

Here, $w_{\mathrm{i}}$ is a relative weight of $\Lambda, \Sigma^{0}$ hyperons and ${ }_{\Lambda}^{12} \mathrm{~B}$ ground state events, $p_{i}^{\prime}$ is a functional weight, $M^{\text {calc }}$ is the calculated mass and $M^{P D B}$ is a known mass from the Particle Data Book [3]. The functional weight $p_{i}^{\prime}$ is described by the function $p_{i}^{\prime}=\exp \left(-\beta_{i} \Delta M_{i}^{2}\right) /\left[\exp \left(-\beta_{i} \lambda_{i}\right)+\exp \left(-\beta_{i} \Delta M_{i}^{2}\right)\right] \quad$ with $\quad$ variables $\quad \beta \approx 0.5 \sigma^{2} \quad$ and $\beta \approx 2.5 \sigma^{2}$ that depend on the expected missing mass resolution $\sigma$.

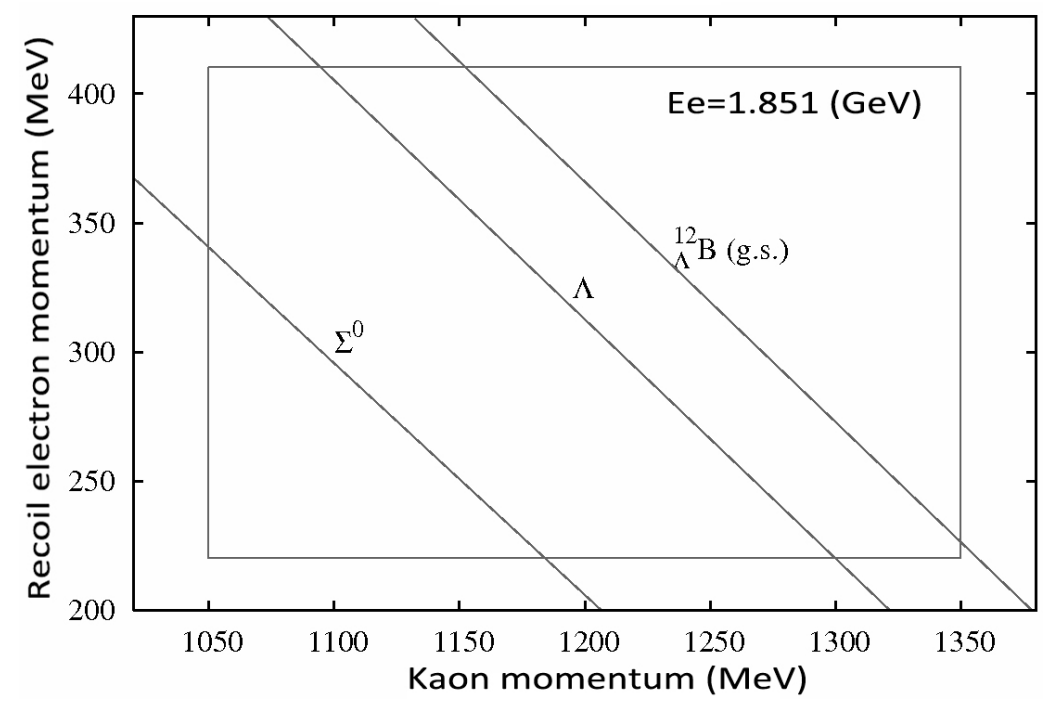

Figure 4.6.2 Momentum correlation between recoil electron and kaon [56]. 
The next step is to minimize the $\chi^{2}$. It is done by the nonlinear least square method. That allows optimization of the momentum reconstruction matrix elements. Further, one fits the ground state binding energy of ${ }_{\Lambda}^{12} \mathrm{~B}$ and using its value goes back to the beginning of iteration. The procedure runs in a loop until the minimum of $\chi^{2}$ is reached. It has been worked out by L. Yuan and L. Tang and details of the procedure can be found in [76].
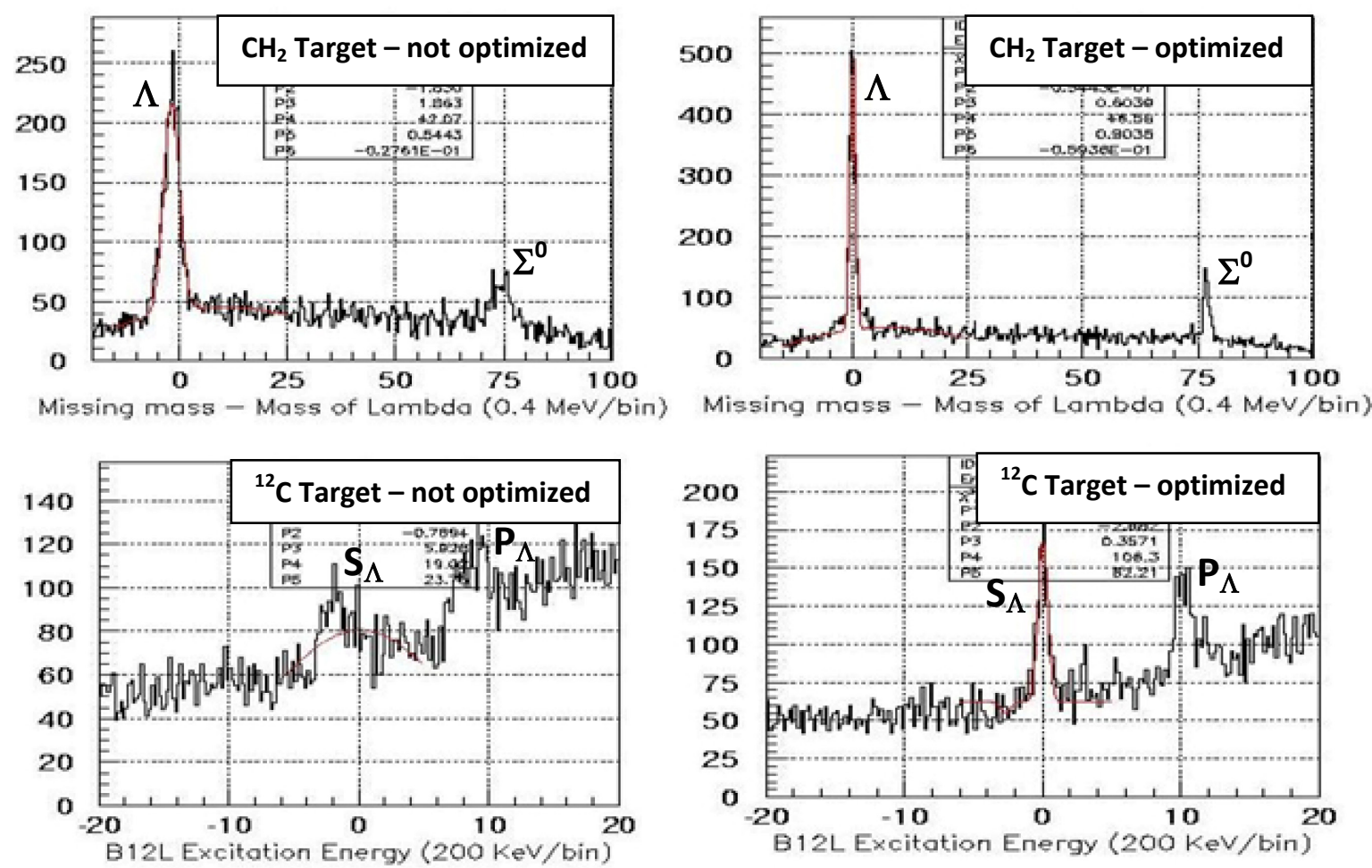

Figure 4.6.3 Missing mass spectra before (left) and after (right) matrix optimization for $\mathrm{CH}_{2}$ and ${ }^{12} \mathrm{C}$ targets [56]. 
An example of the missing mass spectra for $\mathrm{CH}_{2}$ and ${ }^{12} \mathrm{C}$ targets before and after optimization is shown in Fig. 4.6.3. As we can see, the resolution of the peaks has been significantly improved.

\subsubsection{Raster corrections}

For some of the targets the beam raster was used to avoid overheating and burning of the target material. In the reconstruction procedure the presence of the raster has to be accounted for, otherwise the introduced beam position offset at the target will negatively affect the resolution of the missing mass spectra. To perform the raster correction the information from the raster magnets that steer the beam in $X$ and $Y$ directions, is used. In the reconstruction process the raster correction function only depends on beam position.

It is described by the function $g\left(x_{t a r}, y_{t a r}\right)$, such that the adjusted relative momentum becomes

$$
\delta_{p}=f\left(x_{f p}, x_{f p}^{\prime}, y_{f p}, y_{f p}^{\prime}\right)+g\left(x_{t a r}, y_{t a r}\right)
$$

\subsection{Calibration spectra}

The missing mass spectra from $\mathrm{CH}_{2}$ and ${ }^{12} \mathrm{C}$ targets, used for calibration, are shown in the figures 4.7.1 and 4.7.2. The first figure contains a $M_{H}-M_{\Lambda}$ spectrum with a $0.2 \mathrm{MeV}$ per bin scale for the $\mathrm{CH}_{2}$ target. The second spectrum shows the $150 \mathrm{keV}$ per bin scaled binding energy of ${ }_{\Lambda}^{12} \mathrm{~B}$ obtained with the ${ }^{12} \mathrm{C}$ target. 
As we mentioned in section 4.6.2, we use the $\Lambda$ and $\Sigma^{0}$ missing- mass spectra from a $\mathrm{CH}_{2}$ target and the ground state of ${ }_{\Lambda}^{12} \mathrm{~B}$. In the calibration routine the fit values of these peaks are compared with well known $\Lambda$ and $\Sigma^{0}$ masses $\left(m_{\Lambda}=1115.683 \pm 0.006\right.$ $\mathrm{MeV}$ and $\left.m_{\Sigma}=1192.642 \pm 0.024 \mathrm{MeV}\right)$ and binding energy of ${ }_{\Lambda}^{12} \mathrm{~B}\left(B_{\Lambda}=11.37 \pm 0.06\right.$ $\mathrm{MeV})$.

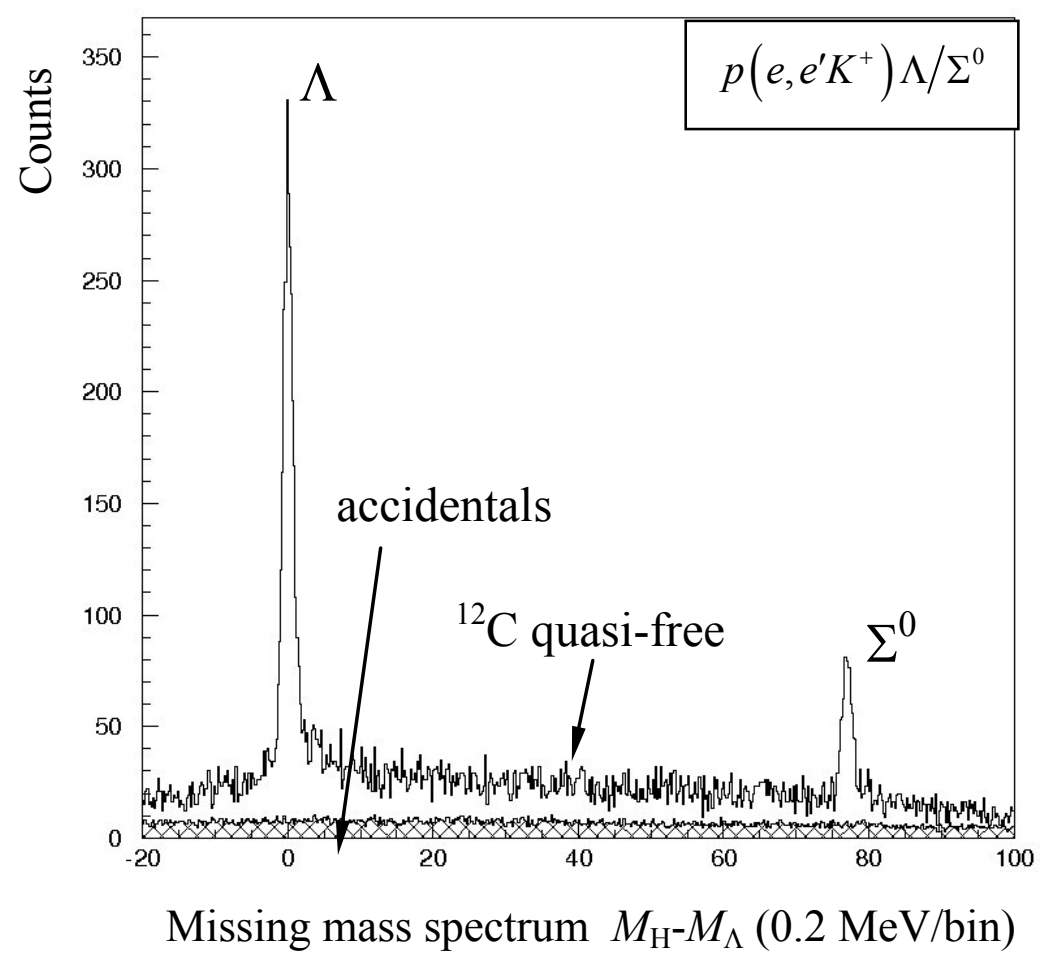

Figure 4.7.1 $\Lambda$ and $\Sigma^{0}$ peaks on missing mass spectrum of $\mathrm{CH}_{2}$ target.

These spectra have been obtained by applying the final reconstruction matrix and placing the \pm 1 ns limiting cut around the true coincidence time. The background part of it, presented by shaded area, has been found by taking the average of eight RF time peaks in the accidental region and scaling accordingly. Figure 4.7.1 clearly shows the $\Lambda$ and $\Sigma^{0}$ peaks, separated by $\approx 77 \mathrm{MeV}$. The quasi-free distribution between the peaks is formed 
by the hyperon production from carbon. The resolution of the peaks is close to $1.5 \mathrm{MeV}$. The statistics of the peaks has been greatly increased since the previous HNSS experiment (Fig. 4.7.3).

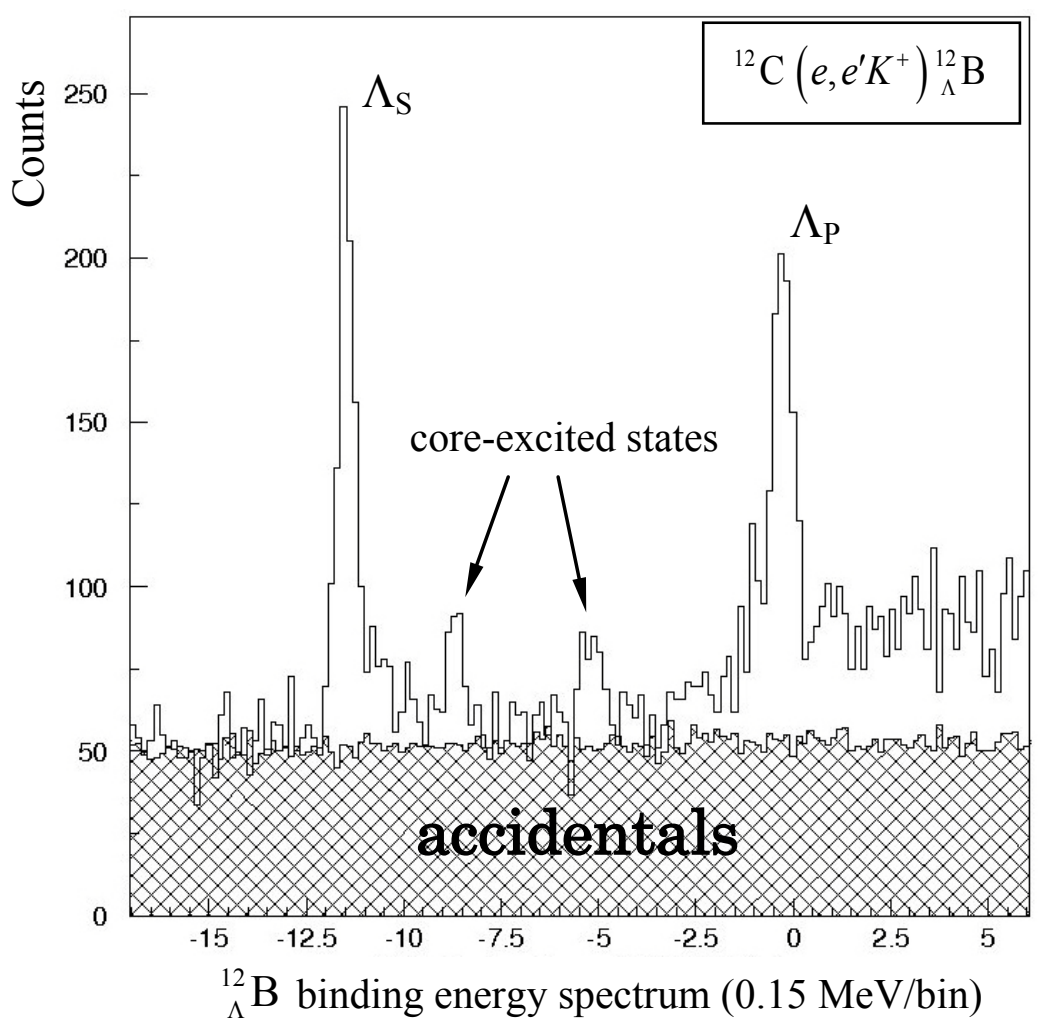

Figure 4.7.2 Binding energy $\left(-B_{\Lambda}\right)$ spectrum of ${ }_{\Lambda}^{12} \mathrm{~B}$ with $150 \mathrm{keV} / \mathrm{bin}$ scale. 


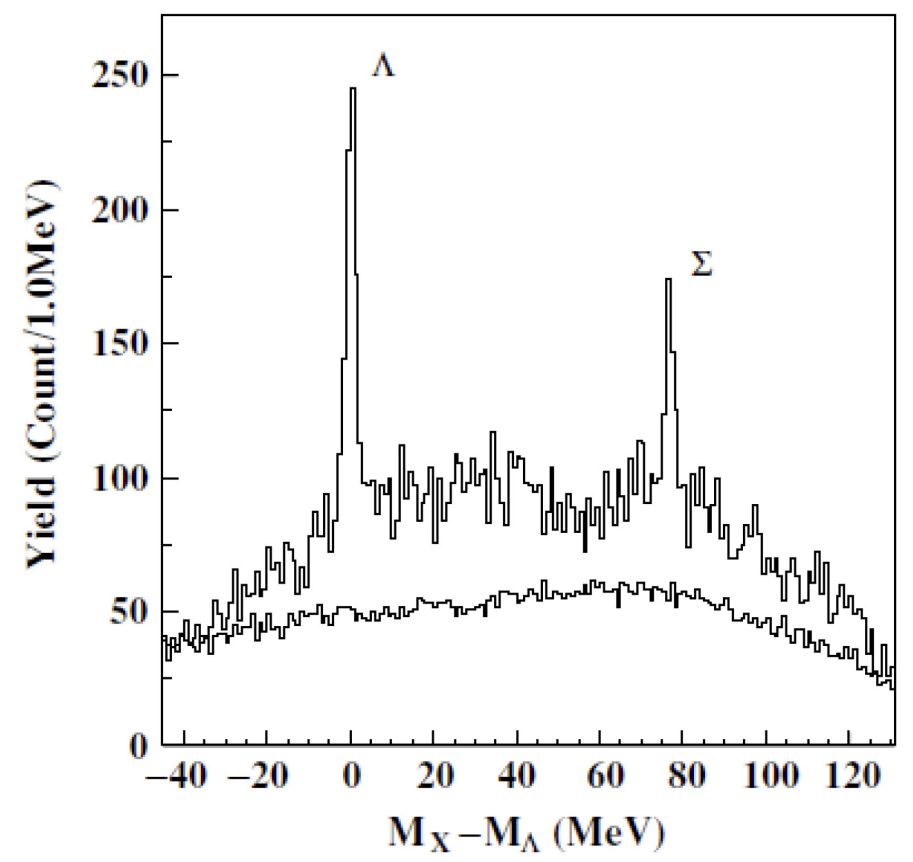

Figure 4.7.3 Missing mass spectrum on $\mathrm{CH} 2$ target in HNSS experiment [12].

The binding energy spectrum of ${ }_{\Lambda}^{12} \mathrm{~B}$, presented in Fig. 4.7.2, contains two clear peaks, approximately separated by $11 \mathrm{MeV}$. These peaks correspond to the $\Lambda$ occupying the $s$ and $p$ shell with the remaining nuclear core in the lowest excitation state. There are also the core-excited states present in the spectrum between the two peaks. There, $\Lambda$ is in the $s$ state, while the nuclear core is in an excited state. The detailed description of the ${ }_{\Lambda}^{12} \mathrm{~B}$ spectrum, obtained by our likelihood approach will be discussed in the next chapter. 


\section{CHAPTER 5}

\section{RESULTS AND DISCUSSIONS}

In this chapter the experimental mass spectra of ${ }_{\Lambda}^{12} \mathrm{~B}$, neutron rich ${ }_{\Lambda}^{7} \mathrm{He}$ hypernuclei, and medium mass ${ }_{\Lambda}^{28} \mathrm{Al}$ are presented. The spectra have been obtained by the application of the earlier described likelihood approach to particle identification in the HKS arm. The chapter also shows a comparison of the ${ }_{\Lambda}^{12} \mathrm{~B}$ spectra independently extracted with the help of both likelihood and standard PID methods. The efficiency of the likelihood method is analyzed and conclusions are drawn. The detailed explanation of the spectroscopy is given and the comparison of experimental results to theoretical calculations is made.

\subsection{Hypernuclear mass spectrum of ${ }_{\Lambda}^{12} \mathrm{~B}$}

\section{Spectrum from likelihood PID}

The hypernuclear production for the ${ }^{12} \mathrm{C}$ target follows the reaction ${ }^{12} \mathrm{C}+e \rightarrow e^{\prime}+K^{+}+{ }_{\Lambda}^{12} \mathrm{~B}$. The data on ${ }^{12} \mathrm{C}$ were taken at different times throughout the data acquisition period. The conditions of the experiment, like trigger thresholds and logic, were constantly changing in an attempt to improve the efficiency of the data acquisition. The current of the electron beam was changed multiple times and ranged between 10 and $30 \mu \mathrm{A}$. Sometimes a current of around $5 \mu \mathrm{A}$ was used for testing purposes. The grouping of the trigger was periodically turned $\mathrm{ON}$ and $\mathrm{OFF}$, and the discriminator threshold voltages of the PID 
trigger of the HKS detector package were adjusted several times to maximize the kaon yield. These changes resulted in variations of the kaon and other particle yields and a difference in the detectors' performances. To account for these changes the ${ }^{12} \mathrm{C}$ data had to be split in parts. In our analysis with likelihood PID we divided the data into 20 sets. For each set the particles' yield fractions were obtained and the positions of the true coincidence time peak were found. Because the efficiencies of both aerogel (Chapter 3) and water Cherenkov [54] detectors have been gradually changing with time, several parameter files, necessary for the likelihood method, were created. In total we created five parameter files to analyze the ${ }^{12} \mathrm{C}$ data. These files included flags and parameters for pdf distributions for each particle and each detector together with the corresponding segments used in the PID analysis. The details of these ${ }^{12} \mathrm{C}$ data sets are shown in Appendix A. The obtained hypernuclear binding energy spectrum of ${ }_{\Lambda}^{12} \mathrm{~B}$ is shown in Fig. 5.1.1. On the horizontal axis the $\Lambda$ binding energy is calculated as $B_{\Lambda}(g s)=M_{A}+M_{\Lambda}-M_{Y}$. The counts are shown on the vertical axis. 


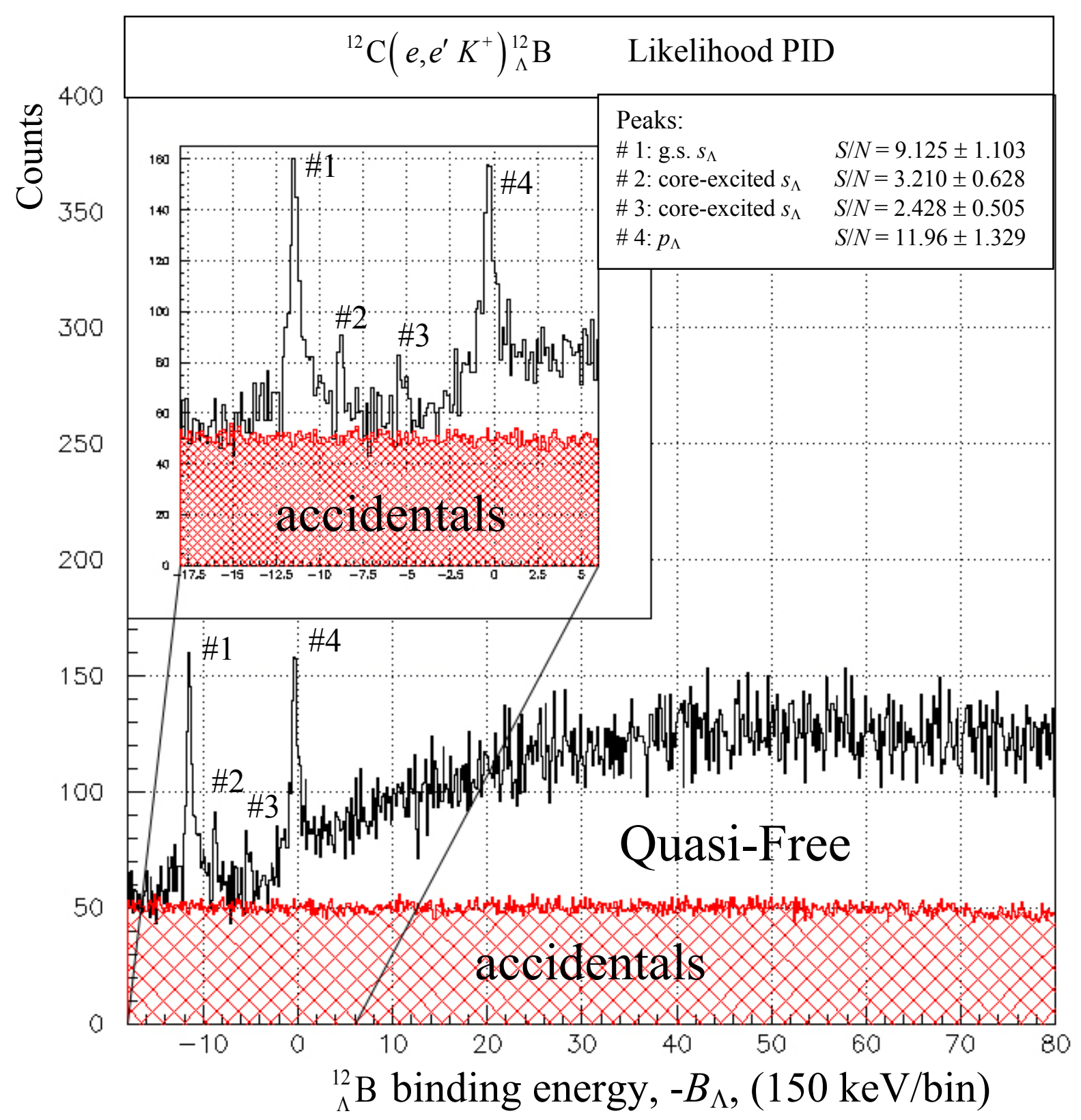

Figure 5.1.1 Binding energy spectrum $\left(-B_{\Lambda}\right)$ of ${ }_{\Lambda}^{12} \mathrm{~B}$ hypernucleus obtained by likelihood PID method. The uncertainties of the peak $S / N$ ratios are statistical.

We can see three prominent distributions: two narrow peaks corresponding to the core nucleus ${ }^{11} \mathrm{~B}$ in its ground state and the $\Lambda$ occupying the $s(\# 1)$ and $p(\# 4)$ shells, and a wide quasi-free distribution. The insert shows the bound region in more detail. Peaks \#2 
and \#3 are interpreted as excitation of the ${ }^{11} \mathrm{~B}$ core with the $\Lambda$ in the $s$-shell. Such states are called core-excited. For each of the peaks the signal to noise ratio has been calculated by the following formula:

$$
S / N=\frac{(S+A)-A}{\sqrt{A}} .
$$

Here $(S+A)$ corresponds to a distribution that contains both true and accidental coincidence events, which survive the \pm 1 ns limiting cut on coincidence time (see Fig. 4.2.3). The accidental distribution, $A$, was found by cutting on the area outside of the true coincidence events. For each of the four peaks a Gaussian fit was applied and the $S / N$ ratio has been found within $\pm 3 \sigma$ of the peak's center. The $S / N$ values together with the statistical uncertainties are shown in the figure on the right top side.

\section{Spectrum from hard-cut PID}

The spectrum shown of the Fig. 5.1.1 can also be found by application of the standard hard-cut PID method. Figure 5.1.2 shows the ${ }_{\Lambda}^{12} \mathrm{~B}$ binding energy obtained with the identical acceptance and coincidence time cuts, but standard approach to PID instead of the likelihood method. The detailed cut conditions are presented in Appendix B. The features of the distribution are very similar to those observed in Fig. 5.1.1 


\section{Spectra comparison}

The comparison of the $S / N$ ratios extracted by Eq. (5.1.1) from the ${ }_{\Lambda}^{12} \mathrm{~B}$ spectra, which was obtained individually by both methods, is shown in Fig. 5.1.3. The horizontal axis is used to designate the peak numbers, which correspond to the notation made in Figs. 5.1.1 and 5.1.2. For standard hard-cut PID method, (blue colored diamonds), the numbers were $1,2,3,4$, and 5. In case of the likelihood PID method, (red colored circles), the numbers were offset by 0.15 for the purpose of simplifying the visual comparison. For each of the points, from 1 to 4 , the $S / N$ ratio has been found for yields within $\pm 3 \sigma$ of the peak centroid, while point 5 does not use any particular peak and includes the total yield between $-18 \mathrm{MeV}$ and $6 \mathrm{MeV}$.

The signal to noise ratios, shown on the figure, are in relatively good agreement. Since both methods sampled the same data, where the only difference was in the total number of events, their statistical uncertainties are correlated and cannot be directly compared to each other. The values for the hard-cut method slightly exceed the likelihood ones. This means that the efficiency of the likelihood PID method does not surpass the hard-cut approach despite the fact that, from the comparison of the beta spectrum in Chapter 4 , an increase in efficiency of approximately $19 \%$ has been expected. To further investigate the efficiency of the likelihood method we introduce a modified condition for likelihood PID:

$$
\left(L_{K}>k \cdot L_{p}\right) \&\left(L_{K}>k \cdot L_{\pi}\right)
$$




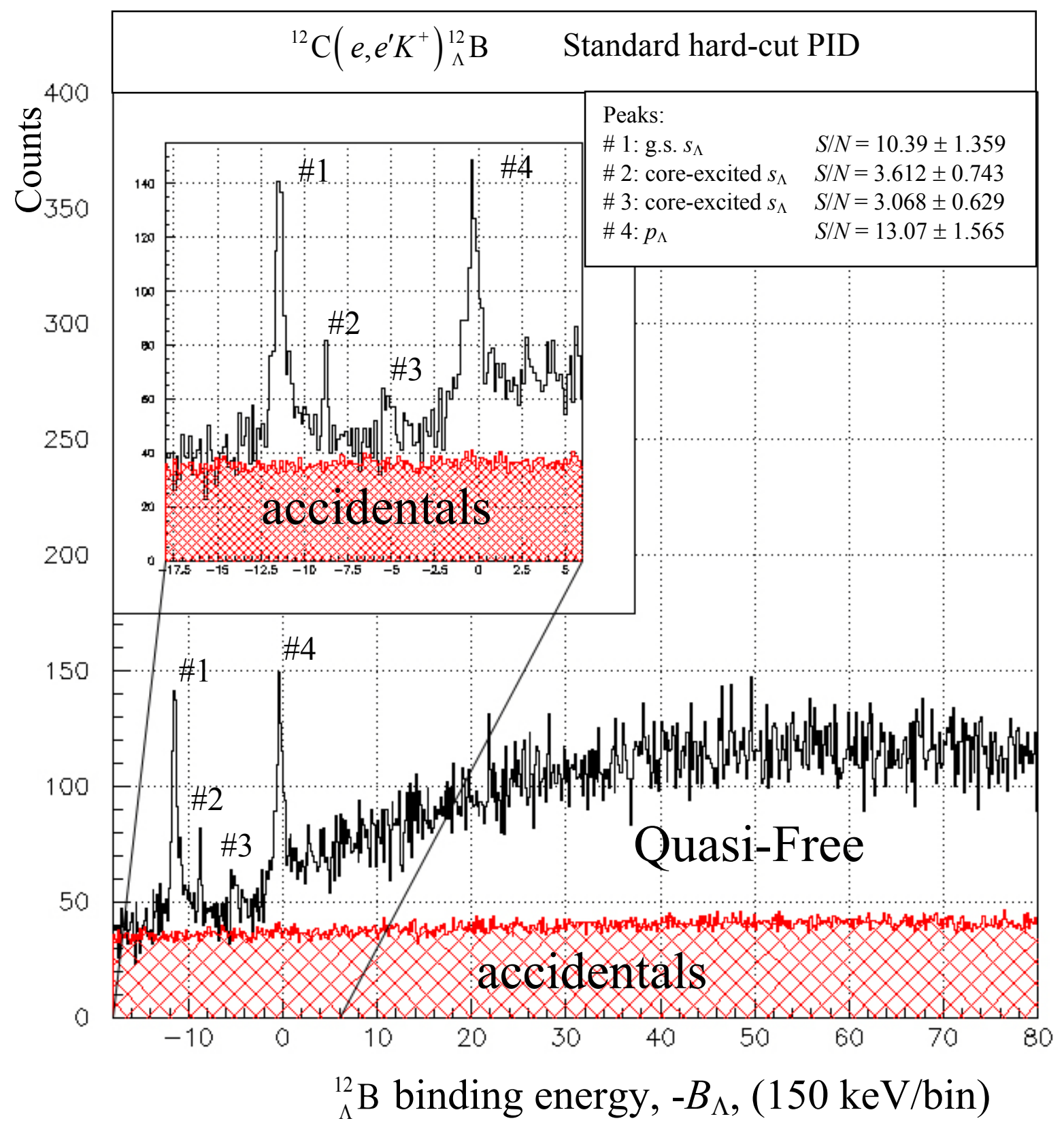

Figure 5.1.2 Binding energy spectrum $\left(-B_{\Lambda}\right)$ of ${ }_{\Lambda}^{12} \mathrm{~B}$ hypernucleus obtained by standard hard-cut PID method. The uncertainties of the peak $S / N$ ratios are statistical. 


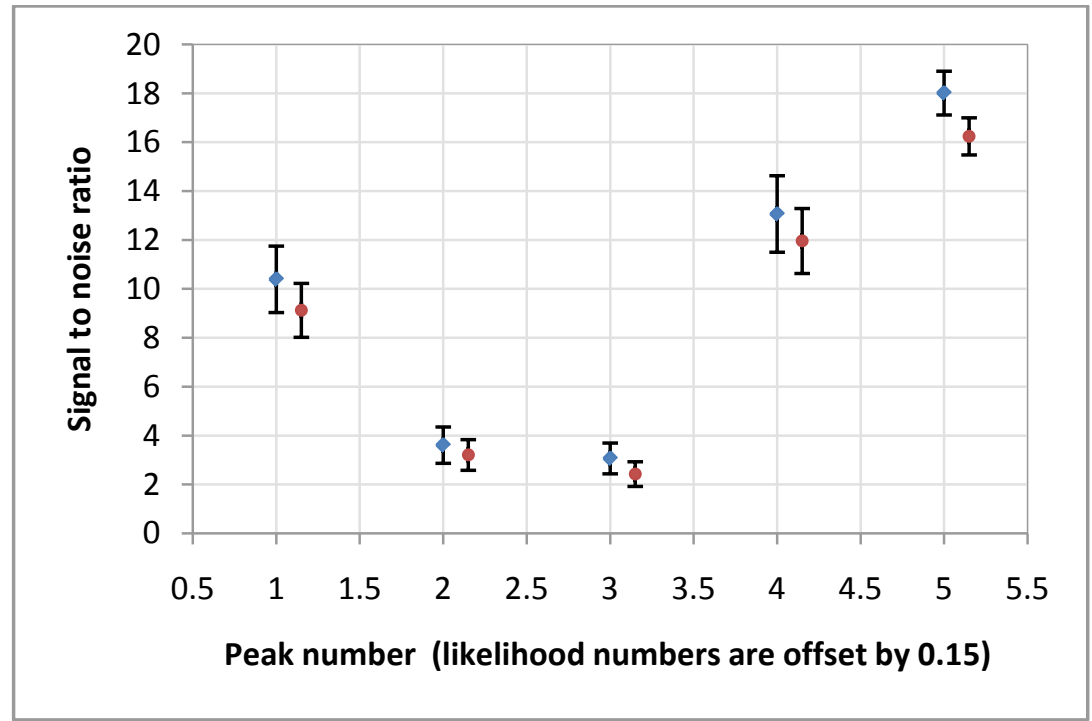

Figure 5.1.3 The comparison of signal to noise ratio for hypernuclear states obtained by hard-cut (blue diamonds) and likelihood (red circles) PID techniques.

The additional coefficient $k$ is used to inexplicitly introduce a cut on the likelihood variable $L_{K}$ as it is shown in Fig. 5.1.4. Because of the normalization of the likelihood values, the kaon likelihood $L_{K}$ ranges from 0 to 1 . The $k=1$ condition, which corresponds to Equation (4.2.22), cuts the likelihood approximately in the middle. Since we differentiate between three particles, the likelihood is supposed to be cut at approximately 0.33 , provided that the particles' yields are statistically independent. However, in our case, two out of three particle hypotheses are always strongly correlated. The correlations of the $L_{K}$ vs. $L_{p}, L_{K}$ vs. $L_{\pi}$, and $L_{p}$ vs. $L_{\pi}$ have been plotted in Fig. 5.1.5. As we can see, the events on each of the plots are mostly distributed on the sides of triangle. If all three particles were equally correlated, the triangle would get filled evenly. 


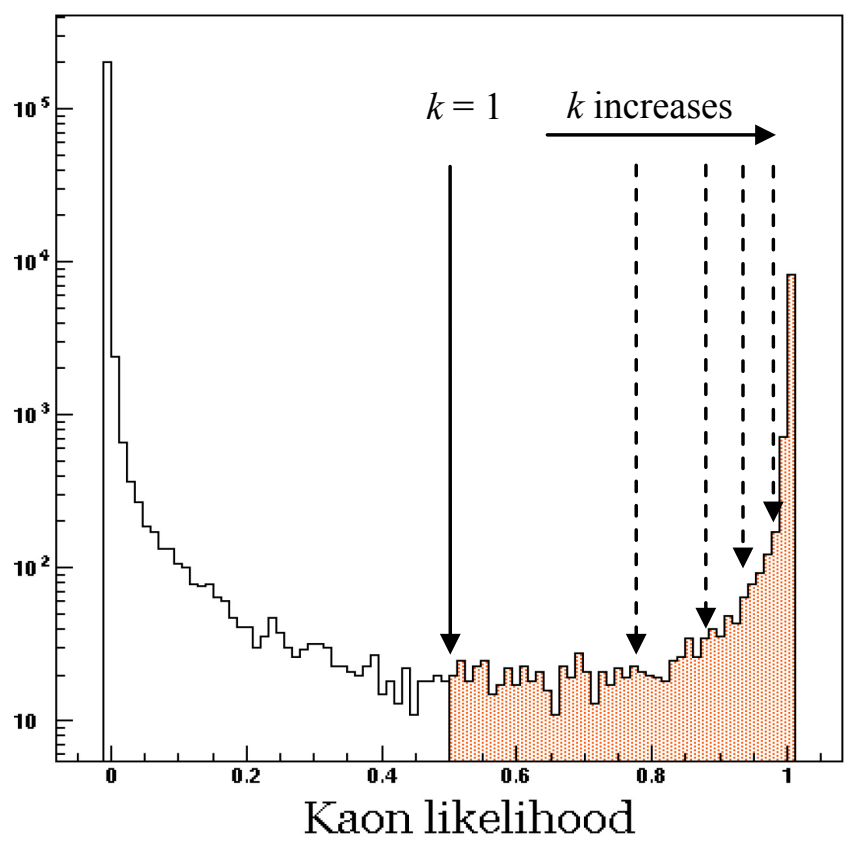

Figure 5.1.4 Hard cut via variable $k$ on kaon likelihood $L_{K}$.

Following the Equation (5.1.2) the scan of the ${ }_{\Lambda}^{12} \mathrm{~B}$ spectra has been performed for each of the four peaks. The coefficient $k$ was increased in each iteration by 500 . The results of the scan are shown in Fig. 5.1.6. The plots display the $S / N$ ratios estimated by Eq. (5.1.1) with statistical uncertainties. The solid red lines on the plots correspond to the $S / N$ ratio of the spectrum obtained by the standard PID approach. The dashed red lines are the statistical uncertainties. As we can see from the plots the likelihood based $S / N$ ratios do not reach the red line for all of the peaks except the second core-excited peak. While in the first iteration, when $k=500$, some increase is observed, further the plots are almost flat. Therefore the $S / N$ ratio of the missing mass spectrum obtained by the likelihood method is not better than the one calculated by the standard hard-cut technique. 
To determine if the introduction of the $k$ coefficient in the likelihood condition can improve the total statistics of the true coincident events, we plot the $(S+A)-A$ versus coefficient $k$. The plots are shown in Fig. 5.1.7. As can be seen from the plots, the signal for all of the peaks has a tendency to decrease with rise of $k$ value. To be able to emphasize the peaks, we have to keep the true signal, $S$, as statistically significant as possible. That apparently corresponds to point $k=1$.
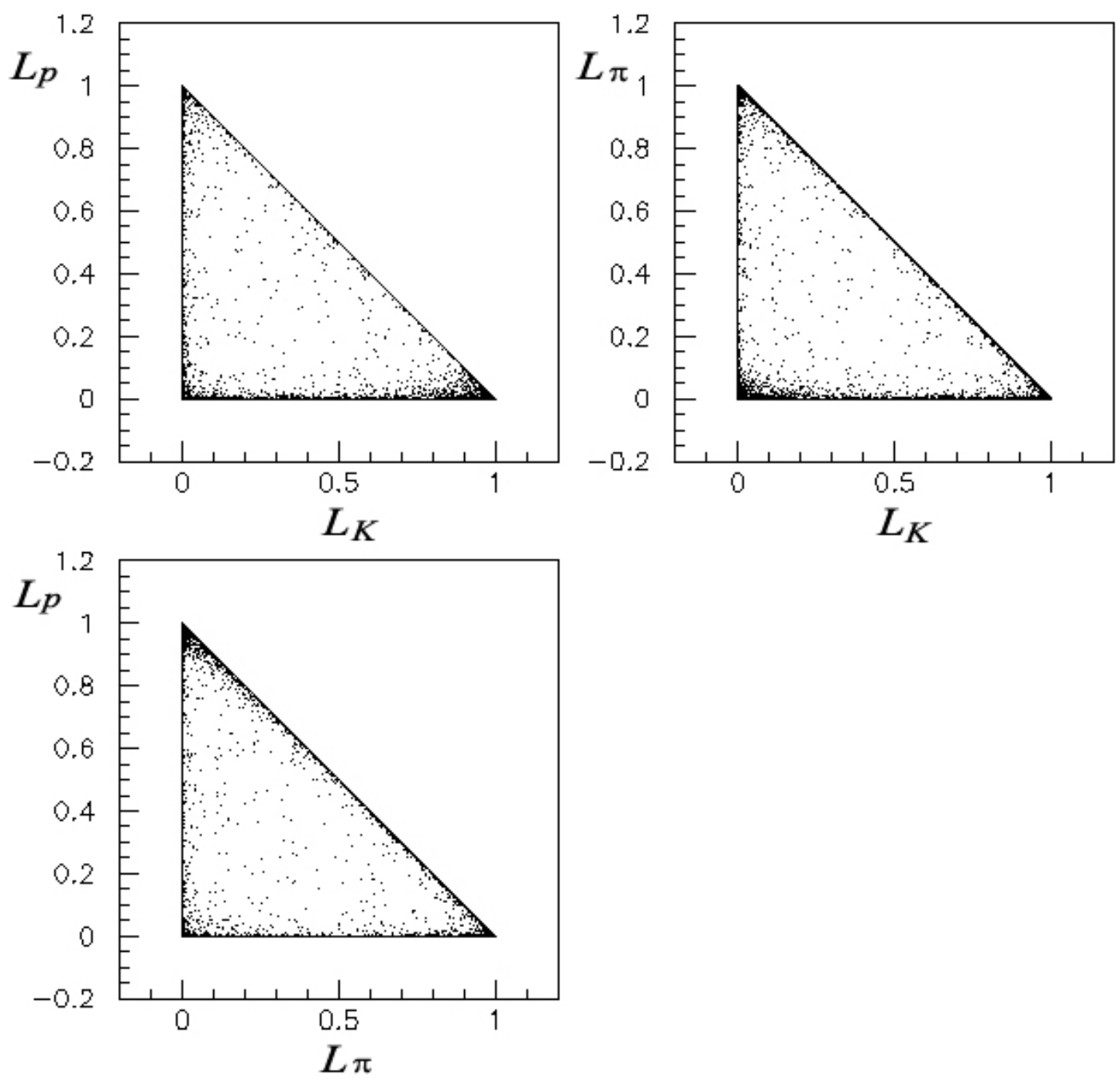

Figure 5.1.5 Likelihood correlations. 


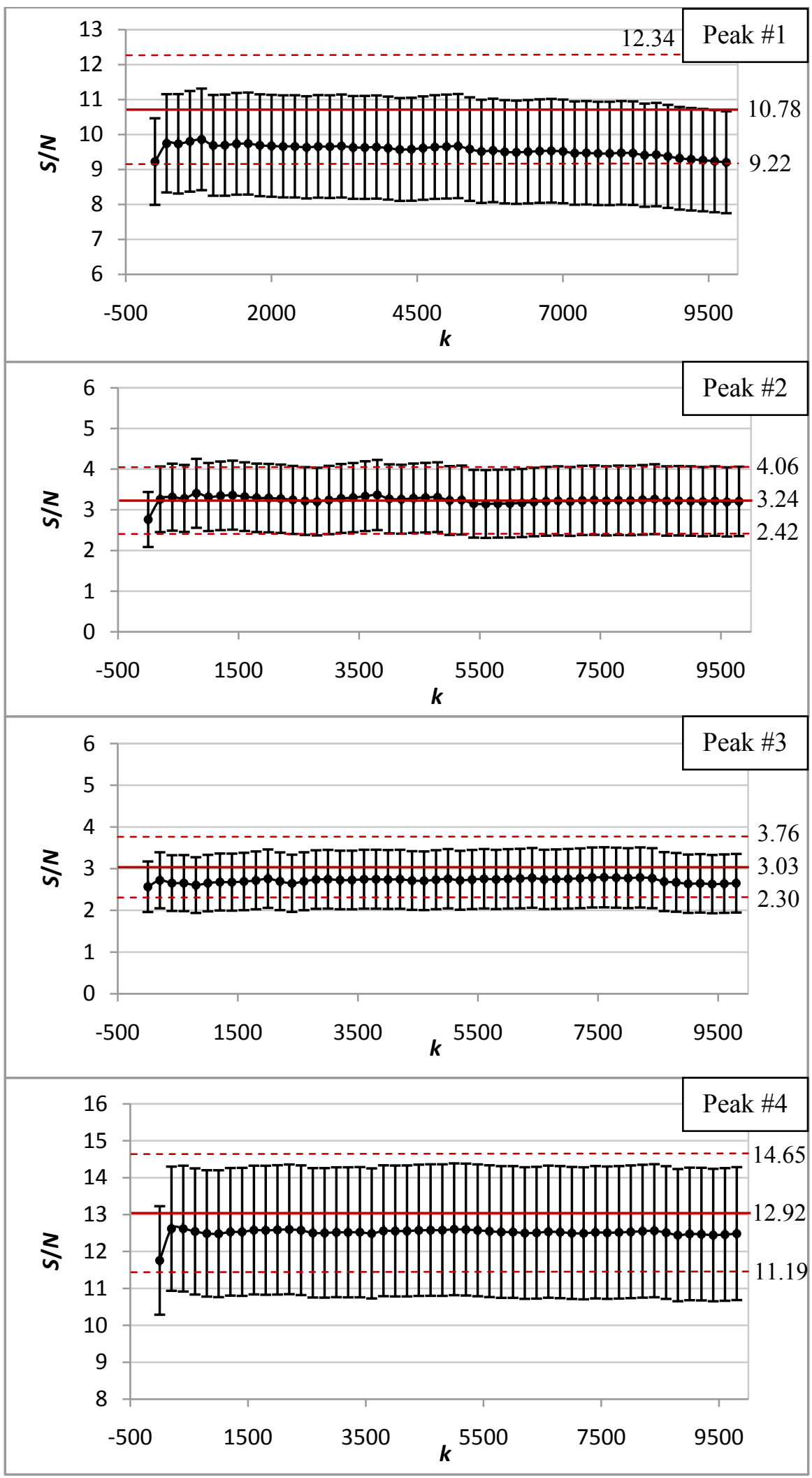

Figure 5.1.6 Scan of the ${ }_{\Lambda}^{12} \mathrm{~B}$ spectra for different coefficient $k$ in likelihood PID condition. The red lines correspond to $S / N$ ratio of the standard PID method. 


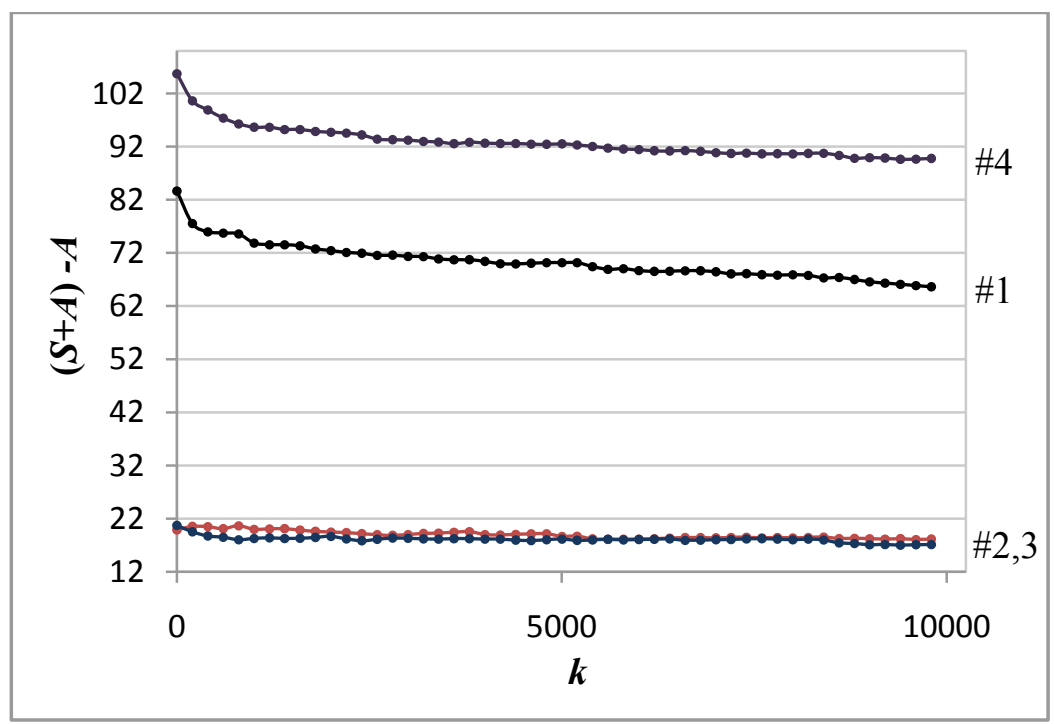

Figure 5.1.7 True coincident events versus coefficient $\mathrm{k}$ for peaks 1, 2, 3, and 4.

The side to side comparison of the ${ }_{\Lambda}^{12} \mathrm{~B}$ spectra obtained by likelihood PID (with $k=1)$ and standard hard-cut PID methods is shown in Fig. 5.1.8. The red colored plot on the left top side of the figure presents the spectrum extracted by application of the likelihood PID, while the blue colored plot on the right top side shows the spectrum found by the hard-cut technique. As can be observed, with the likelihood approach we gain statistics in accidental signal $A$ (left bottom plot) while true coincidence signal $S$ (right bottom plot) nearly stays the same. The summarized numerical comparison of the spectra is given in the Table 5.1.1.

Table 5.1.1 Comparison of ${ }_{\Lambda}^{12} \mathrm{~B}$ spectra created by likelihood and hard-cut PID techniques.

\begin{tabular}{ccccc}
\hline Peak \# & \multicolumn{2}{c}{$S / N$ ratio } & \multicolumn{2}{c}{$(S+A)-A$ within $3 \sigma$ of peak's mean value } \\
& Likelihood PID & Hard-cut PID & Likelihood PID & Hard-cut PID \\
\hline 1 & $9.125 \pm 1.103$ & $10.39 \pm 1.359$ & $83.656 \pm 15.753$ & $82.113 \pm 14.074$ \\
\hline 2 & $3.210 \pm 0.628$ & $3.612 \pm 0.743$ & $19.863 \pm 11.101$ & $19.750 \pm 9.708$ \\
\hline 3 & $2.428 \pm 0.505$ & $3.068 \pm 0.629$ & $20.738 \pm 12.307$ & $21.081 \pm 10.854$ \\
\hline 4 & $11.96 \pm 1.329$ & $13.07 \pm 1.565$ & $105.725 \pm 16.355$ & $101.694 \pm 15.020$ \\
\hline
\end{tabular}



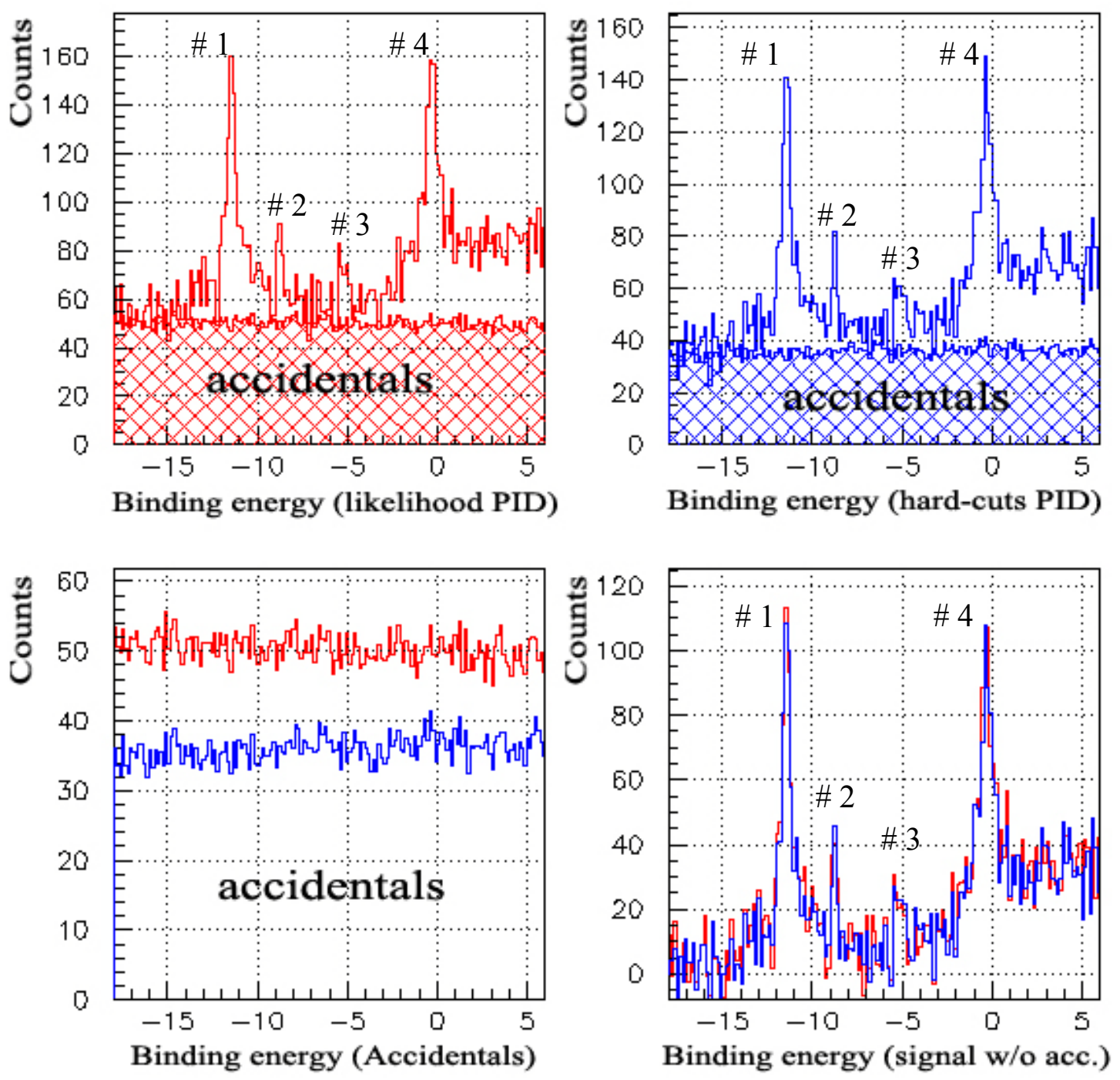

Figure 5.1.8 Side-by-side comparison of ${ }_{\Lambda}^{12} \mathrm{~B}$ spectra found by likelihood and hard-cut PID methods.

In summary, in Chapter 4 we found that the likelihood PID method identifies extra kaons not found by the standard approach but at the same time also misses kaons. From the comparison of the missing mass spectra we conclude that both methods are equally efficient in identifying kaons. The increased yield of accidentals in the likelihood method spectrum indicates a less efficient suppression of protons and pions. Although a 
likelihood technique is commonly believed to be a superior method, the frequent changes to the experimental conditions might have hampered the successful application of the method. It would be a worthwhile to revisit this approach in the future for a more uniform data set.

\subsection{Spectroscopy of ${ }_{\Lambda}^{12} B$ hypernuclei}

\section{Composition of the hypernuclear states}

Because the ${ }^{12} \mathrm{C}\left(e, e^{\prime} K^{+}\right){ }_{\Lambda}^{12} \mathrm{~B}$ reaction employs proton conversion into a $\Lambda$, there is a proton hole- $\Lambda$ particle configuration. To obtain the hypernuclear states of ${ }_{\Lambda}^{12} \mathrm{~B}$, the nuclear states of the ${ }^{11} \mathrm{~B}$ core must be coupled with a $\Lambda$ in the $s$ and $p$ shells. Two diagrams below, Fig. 5.2.1, display the energy levels of ${ }^{11} \mathrm{~B}$ and $\Lambda$ that can be utilized for the composition of the final hypernuclear states.

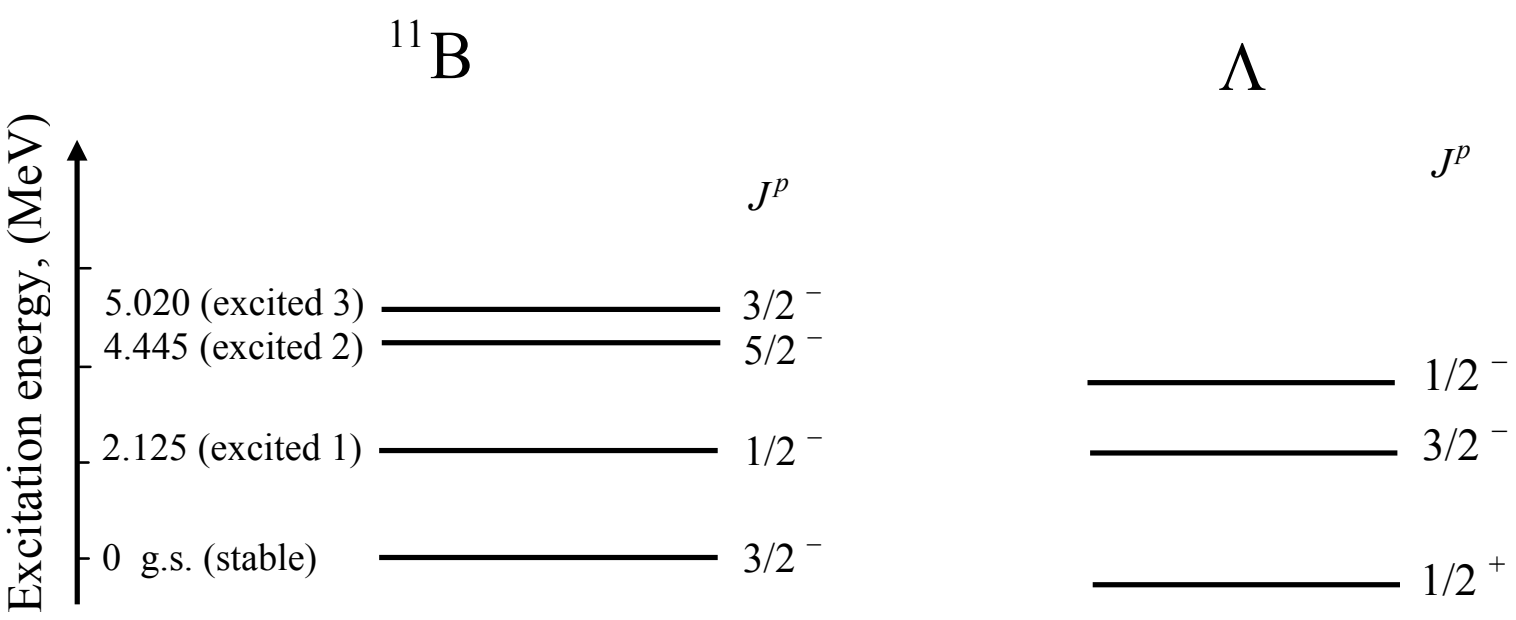

Figure 5.2.1 Schematics of the energy levels of ${ }^{11} \mathrm{~B}$ nuclear host and $\Lambda$ particle. 
The representation of the levels is schematic with arbitrary relative locations of the ${ }^{11} \mathrm{~B}$ and $\Lambda$ states. The ${ }^{11} \mathrm{~B}$ nuclear core contains 5 protons and 6 neutrons and in its ground state has $J^{p}=3 / 2^{-}$. The proton hole configuration means that the proton was removed from the ${ }^{12} \mathrm{C}$ nucleus and substituted by $\Lambda$ hyperon. Now, the $\Lambda$ particle can couple with any of the ground and excited states of ${ }^{11} \mathrm{~B}$. One of the strongly populated states, a substitutional state, would correspond to the $\Lambda$ acquiring the quantum numbers of the $3 / 2$ ground state of the host nucleus. The final hypernuclear states of ${ }_{\Lambda}^{12} \mathrm{~B}$ can be composed by coupling the angular momenta in the following way:

$$
\left|J_{{ }^{11_{B}}}-J_{\Lambda}\right| \leq J_{H} \leq\left|J_{{ }^{11_{B}}}+J_{\Lambda}\right|,
$$

where $J_{H}$ is the angular momentum of the composed hypernucleus. Table 5.2.1 shows some of the possible combinations that can be arranged by condition (5.2.1).

Table 5.2.1 Some of the possible ${ }_{\Lambda}^{12} \mathrm{~B}$ states from coupling by Equation (5.2.1).

\begin{tabular}{ccc}
\hline${ }^{11} \mathrm{~B}, J^{p}($ shell $)$ & $\Lambda, J^{p}($ shell $)$ & ${ }_{\Lambda}^{12} \mathrm{~B}, J^{p}$ \\
\hline $3 / 2^{-}(p$, g.s. $)$ & $1 / 2^{+}(s)$ & $\begin{array}{l}1^{-} \\
2^{-}\end{array}$ \\
\hline $1 / 2^{-}(p$, ex. 1$)$ & $1 / 2^{+}(s)$ & $0^{-}$ \\
& $1 / 2^{+}(s)$ & $2^{-}$ \\
\hline $5 / 2^{-}(p$, ex. $)$ & $1 / 2^{+}(s)$ & $3^{-}$ \\
\hline $3 / 2^{-}(p$, ex.3) & $3 / 2^{-}(p)$ & $2^{-}$ \\
\hline $3 / 2^{-}(p$, g.s. $)$ & $1 / 2^{-}(p)$ & $0^{+}$ \\
\hline $3 / 2^{-}(p$, g.s. $)$ & & $3^{+}$ \\
\hline
\end{tabular}


It is important to note that Table 5.2.1 is somewhat naive, especially for ${ }^{11} \mathrm{~B}$ coupling to a $\Lambda$ in the $p$-shell. The coupling between the closely spaced states, for example $\Lambda p\left(1 / 2^{-}\right)$ and $\Lambda p\left(3 / 2^{-}\right)$, has to be taken into account. The strengths of the produced states also have to be considered. That might eliminate some of the states and add other states. The hypernuclear states are populated in proportion to the strengths of the proton removal from the states of the target's core nucleus. In the process of ${ }^{12} \mathrm{C}$ converting to ${ }^{11} \mathrm{~B}$, the proton removal strength is the highest for the ${ }^{11} \mathrm{~B}\left(3 / 2^{-}\right)$ground state and is decreasing for the core excited ${ }^{11} \mathrm{~B}\left(1 / 2^{-}\right)$and ${ }^{11} \mathrm{~B}\left(3 / 2^{-}\right)$states. In contrast with these core excited states, the $\Lambda$ coupling with ${ }^{11} \mathrm{~B}\left(5 / 2^{-}\right)$state can be neglected. While considering the strength of the coupling states, one also has to include the cross-section information of the elementary $p\left(e, e^{\prime} K^{+}\right) \Lambda$ reaction. In the forward angle reaction the spin=1 virtual photon results in predominantly spin-flip proton to $\Lambda$ conversion. The non-flip transition exists as well, however, it has a lower probability. Because of that, the states populated with the $\Lambda$ spin-flip transition are the strongest.

The theoretical prediction of the excitation energy, $E_{x}=B_{\Lambda}-B_{\Lambda(g . s .)}$, is given in Table 5.2.2 [77]. Also shown are the theoretical values of the cross section for the kinematics of the previously conducted experiment [78] in Hall A at Jefferson Lab. They will be used as guidance for the strength of the peaks while fitting the data. The crosssection predictions used the Distorted Wave Impulse Approximation (DWIA) framework with the Saclay-Lyon (SLA) model for elementary $p\left(e, e^{\prime} K^{+}\right) \Lambda$ process $[78,79]$. As we can see from the Fig. 5.1.8, there are four significant peaks in the ${ }_{\Lambda}^{12} \mathrm{~B}$ spectrum. Comparing this to the theoretical predictions from Table 5.2.2, the first peak, \#1, 
corresponds to a doublet state $\left(1^{-}, 2^{-}\right)$that is created due to the $\Lambda\left(1 / 2^{+}\right)$in the $s$-shell proton hole coupling to the ${ }^{11} \mathrm{~B}\left(3 / 2^{-}\right) p$-shell ground state. The next two peaks, $\# 2$ and $\# 3$, have doublet configurations as well and belong to the $\Lambda\left(1 / 2^{+}\right)$in the $s$-shell coupling to the nuclear core in excited $1 / 2^{-}$or $3 / 2^{-} p$ states. Peak \#4, contains four closely located hypernuclear states originated from coupling the $\Lambda$ in $1 / 2^{-}$or $3 / 2^{-} p$-shell to the ${ }^{11} \mathrm{~B}\left(3 / 2^{-}\right)$ $p$-shell ground state. All mentioned hypernuclear states are in the bound region of the binding energy spectrum. The last two states predicted in Table 5.2.2. lie in the quasi-free region that is above the $\Lambda$ separation threshold. They result from coupling a $\Lambda$ in the $p$ shell to the first excited $1 / 2^{-}$state of ${ }^{11} \mathrm{~B}$.

Table 5.2.2 Theoretical predictions of excitation energy $E_{x}$ of ${ }_{\Lambda}^{12} \mathrm{~B}$ hypernuclear states $[77,80]$.

\begin{tabular}{ccccc}
\hline${ }^{11} \mathrm{~B}, J^{p}($ shell $)$ & $\Lambda, J^{p}($ shell $)$ & ${ }_{\Lambda}^{12} \mathrm{~B}, J^{p}$ & $E_{x}(\mathrm{MeV})$ & Cross section $\left(\mathrm{nb} / \mathrm{sr}^{2} / \mathrm{GeV}\right)$ \\
\hline \multirow{2}{*}{$3 / 2^{-}(p$, g.s. $)$} & $1 / 2^{+}(s)$ & $1^{-}$ & 0.0 & 1.02 \\
& & $2^{-}$ & 0.14 & 3.66 \\
\hline \multirow{2}{*}{$1 / 2^{-}(p$, ex. 1$)$} & $1 / 2^{+}(s)$ & $0^{-}$ & - & - \\
& & $1^{-}$ & 2.67 & 1.54 \\
\hline \multirow{2}{*}{$3 / 2^{-}(p$, ex.3) } & $1 / 2^{+}(s)$ & $2^{-}$ & 5.74 & 0.58 \\
& & $1^{-}$ & 5.85 & 0.18 \\
\hline \multirow{2}{*}{$3 / 2^{-}(p$, g.s. $)$} & $1 / 2^{-}(p) \otimes 3 / 2^{-}(p)$ & $1^{+}$ & 10.48 & 0.24 \\
& $1 / 2^{-}(p)$ & $2^{+}$ & 10.52 & 0.12 \\
& $3 / 2^{-}(p)$ & $3^{+}$ & 11.05 & 1.43 \\
\multirow{2}{*}{$1 / 2^{-}(p$, ex.1) } & $3 / 2^{-}(p)$ & $2^{+}$ & 12.95 & 2.19 \\
\hline
\end{tabular}




\section{Spectroscopy}

To extract the $\Lambda$ binding and excitation energies, we apply the following fit:

$$
f(x)=G_{\mathrm{I} . \mathrm{a}}+R V_{\mathrm{I} . \mathrm{b}}+G_{\mathrm{II}}+G_{\mathrm{III}}+G_{\mathrm{IV}}+G_{\mathrm{V} . \mathrm{a}}+G_{\mathrm{V} . \mathrm{b}}+G_{\mathrm{V} . \mathrm{c}}+G_{\mathrm{VI}}+Q F+B k g
$$

Here, $G$ represents a Gaussian, $R V$ means Right Voigt defined by Equation (4.2.9), $Q F$ is a quasi-free distribution, and Bkg is a background. Figure 5.2.2 shows the results of the fit. Peak \#I contains $G_{\text {I.a }}$ and $R V_{\text {I.b }}$ functions. Peaks \#II, \#III and \#IV use $G_{\text {II, }} G_{\text {III, }}$ and $G_{\mathrm{IV}}$, respectively. Peak \#V is composed of the three Gaussians $G_{\mathrm{V} . \mathrm{a}}, G_{\mathrm{V} . \mathrm{b}}$, and $G_{\mathrm{V} . c}$. In the selected energy range $(-18.0-6.0 \mathrm{MeV})$, the $Q F$ distribution was fitted by a square root function and the background was approximated to be linear.

The peak \#I.b was chosen to be fit by a Voigt function due to its long tail on the right side. At this time, this tail is not well understood. Radiative effects associated with such physical processes as multiple scattering and bremsstrahlung in the target, hard scattering from Møller ep elastic and inelastic processes should lead to a tail to the right of each peak. However, we expect it to be much smaller in magnitude. None of the existing models predicts a state in this region. Thus, it could also be attributed to a statistical fluctuation or could be the result of a short period of data taking with either an offset in the beam energy or a spectrometer setting.

It is important to note that we observe four statistically significant peaks: \#I, \#II, $\# \mathrm{IV}$, and $\# \mathrm{~V}$. In order to obtain the best fit, peak \#III has been intentionally added to the fit function using the theoretically predicted position and strength. For the same reason peaks \#I.a, \#V.a, \#V.b, \#VI were used as well. Their relative positions and strengths have 
been adjusted according to Table 5.2.2. As an experimental result, we only chose the mean values of the peaks that statistically contribute the most. These are \#I.b, \#II, \#IV and \#V.c. The comparison of experimental results for binding energies with theoretical predictions $[77,80]$ of excitation energies is presented in Table 5.2.3.

There were two types of uncertainties that contributed to the total systematic uncertainties: kinematics and optics. Kinematics uncertainties resulted from fitting uncertainties of the $\Lambda$ and $\Sigma$ peaks in the $\mathrm{CH}_{2}$ target spectrum, while the optics uncertainties correspond to uncertainties in the reconstruction-matrix-minimization procedure. Within the collaboration [56] the uncertainties associated with kinematics were reported to be about $\pm 100 \mathrm{keV}$, while the optics-related uncertainties were \pm 50 $\mathrm{keV}$ [81]. The systematic uncertainties shown in the Table 5.2.3 were estimated as the quadrature of the kinematics and optics uncertainties.

In the fitting procedure the widths of the peaks were kept fixed within a limiting interval of $0 \leq \sigma_{F W H M} \leq 0.42(\mathrm{MeV})$. That allowed higher precision in finding the mean values of the peaks. 

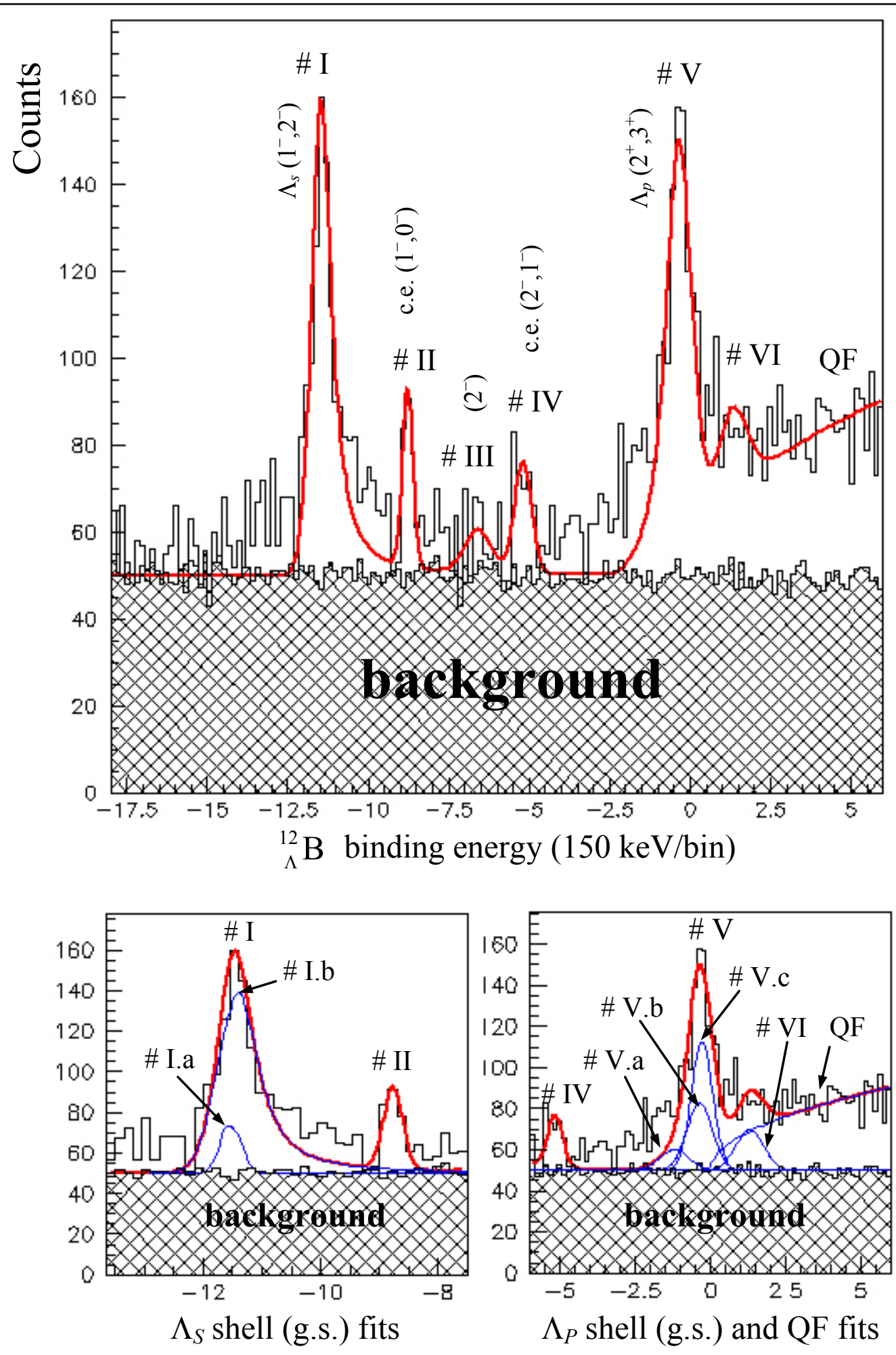

Figure 5.2.2 Fit results for ${ }_{\Lambda}^{12} \mathrm{~B}$ binding energy $\left(-B_{\Lambda}\right)$ spectrum obtained by the likelihood PID method. 
Table 5.2.3 Comparison of theoretical $[77 ; 80]$ and experimental excitation energies for ${ }_{\Lambda}^{12} \mathrm{~B}$ spectrum.

\begin{tabular}{|c|c|c|c|c|c|c|c|c|}
\hline \multirow{2}{*}{${ }^{11} \mathrm{~B}, J^{p}$ (shell) } & \multirow{2}{*}{$\Lambda, J^{p}$ (shell) } & \multirow{2}{*}{\multicolumn{2}{|c|}{ Peak \# }} & \multirow{2}{*}{${ }_{\Lambda}^{12} \mathrm{~B}, J^{p}$} & \multirow{2}{*}{$\begin{array}{l}\text { Theor. } \\
\text { strength }\end{array}$} & \multicolumn{2}{|c|}{ Excitation $E_{x}(\mathrm{MeV})$} & \multirow{2}{*}{$\begin{array}{c}\text { Binding energy } \\
-B_{\Lambda}(\mathrm{MeV})\end{array}$} \\
\hline & & & & & & Theor & Exp. & \\
\hline \multirow[b]{2}{*}{$3 / 2^{-}(p$, g.s. $)$} & \multirow[b]{2}{*}{$1 / 2^{+}(s)$} & \multirow[b]{2}{*}{ \# I } & $\mathrm{a}$ & $1^{-}$ & 1.02 & 0.0 & 0.0 & $-11.530 \pm 0.008(\mathrm{st})$ \\
\hline & & & $\mathrm{b}$ & $2^{-}$ & 3.66 & 0.142 & $\begin{array}{c}0.14 \pm 0.031(\mathrm{st}) \\
\pm 0.05(\mathrm{sys})\end{array}$ & $-11.390 \pm 0.030(\mathrm{st})$ \\
\hline \multirow[b]{2}{*}{$1 / 2^{-}(p$, ex. 1$)$} & \multirow[b]{2}{*}{$1 / 2^{+}(s)$} & \multirow{2}{*}{\multicolumn{2}{|c|}{ \# II }} & $0^{-}$ & - & 2.666 & & \\
\hline & & & & $1^{-}$ & 1.54 & 2.670 & $\begin{array}{c}2.76 \pm 0.04(\mathrm{st}) \\
\pm 0.05(\mathrm{sys})\end{array}$ & $-8.766 \pm 0.039(\mathrm{st})$ \\
\hline $5 / 2^{-}(p$, ex. 2$)$ & $1 / 2^{+}(s)$ & \# III & & $2^{-}$ & - & & & $-6.596 \pm 0.221(\mathrm{st})$ \\
\hline \multirow[t]{2}{*}{$3 / 2^{-}(p$, ex. 3$)$} & \multirow[t]{2}{*}{$1 / 2^{+}(s)$} & \multirow{2}{*}{\multicolumn{2}{|c|}{ \# IV }} & $2^{-}$ & 0.58 & 5.743 & $\begin{array}{c}6.34 \pm 0.07(\mathrm{st}) \\
\pm 0.05(\mathrm{sys})\end{array}$ & $-5.186 \pm 0.070(\mathrm{st})$ \\
\hline & & & & $1^{-}$ & 0.18 & 5.850 & & \\
\hline \multirow{4}{*}{$3 / 2^{-}(p$, g.s. $)$} & $3 / 2^{-}(p)$ & & $\bar{a}$ & $2^{+}$ & 0.24 & 10.48 & & $-1.200 \pm 0.028(\mathrm{st})$ \\
\hline & $1 / 2^{-}(p) \otimes 3 / 2^{-}(p)$ & & - & $1^{+}$ & 0.12 & 10.52 & & \\
\hline & $1 / 2^{-}(p)$ & $\# \mathrm{~V}$ & $\mathrm{~b}$ & $2^{+}$ & 1.43 & 10.98 & & $-0.360 \pm 0.013(\mathrm{st})$ \\
\hline & $3 / 2^{-}(p)$ & & $\mathrm{c}$ & $3^{+}$ & 2.19 & 11.05 & $\begin{array}{c}11.25 \pm 0.061(\mathrm{st}) \\
\pm 0.05(\mathrm{sys})\end{array}$ & $-0.280 \pm 0.060(\mathrm{st})$ \\
\hline \multirow{2}{*}{$1 / 2^{-}(p$, ex. 1$)$} & $3 / 2^{-}(p)$ & \multirow{2}{*}{ \# VI } & & $2^{+}$ & 0.91 & 12.95 & & $1.300 \pm 0.077$ (st) \\
\hline & $1 / 2^{-}(p) \otimes 3 / 2^{-}(p)$ & & - & $1^{+}$ & 0.27 & 13.05 & & \\
\hline
\end{tabular}

To estimate the resolution of the peaks, we performed the following analysis. The resolution of the first peak, $\# 1$, is dominated by the width of peak \#I.b, which is described by a Voigt function, (4.2.9). Inside of the fitting routine, the mean value of the peak has been fixed, while the width of the Lorentzian part has been relaxed. Since by definition the Lorentzian function brings along wide tails, it fits relatively well the right side of the ground state peak. That allowed obtaining the width of the Gaussian part, which was $441.58 \pm 54.64 \mathrm{keV}$. Now the resolution of the core excited states, \#2 and \#3, fitted by Gaussians were $387.02 \pm 90.53 \mathrm{keV}$ and $536.99 \pm 131.88 \mathrm{keV}$, respectively. For the last peak \#4 we obtain an energy width of $418.09 \pm 115.29 \mathrm{keV}$, which was taken to be equal to the width of the $3^{+}$state (peak \#V.c). The FWHM resolutions mentioned above are summarized in Table 5.2.4. The peak assignment is given in Table 5.2.3 
Table 5.2.4 Experimental energy resolution of ${ }_{\Lambda}^{12} \mathrm{~B}$ states.

\begin{tabular}{ccc}
\hline Peak & ${ }_{\Lambda}^{12} \mathrm{~B}$ state, $J^{p}$ & Energy width (FWHM), keV \\
\hline$\#$ I.b & $2^{-}$ & $442 \pm 55(\mathrm{st})$ \\
\hline$\# \mathrm{II}$ & $1^{-}$ & $387 \pm 91(\mathrm{st})$ \\
\hline$\# \mathrm{IV}$ & $2^{-}$ & $537 \pm 132(\mathrm{st})$ \\
\hline$\#$ V.c & $3^{+}$ & $418 \pm 115(\mathrm{st})$ \\
\hline
\end{tabular}

\section{Comparison with previous results}

The first reaction spectroscopy of ${ }_{\Lambda}^{12} \mathrm{~B}$ was performed in AGS experiment E907 at BNL in the mid 90's [82,83]. It utilized the charge and strangeness exchange reaction mechanism that had not been previously used, see Fig. 1.5.2 (top-right). This hypernuclear production mechanism corresponds to the $\left(K^{-}+p \rightarrow \Lambda+\pi^{0}\right)$ reaction, described earlier in Equation (1.5.2). A negative kaon beam was produced by the Alternating Gradient Synchrotron (AGS) at BNL and then brought to rest on a thin ${ }^{12} \mathrm{C}$ target resulting in the formation of a neutral $\pi^{0}$ and a recoiling ${ }_{\Lambda}^{12} \mathrm{~B}$ hypernucleus. The experiment utilized the Neutral Meson Spectrometer and active target chambers to detect the total energy of the $\pi^{0}$ with $\approx 0.73 \mathrm{MeV}$ resolution. From the $\pi^{0}$ energy spectrum the ${ }_{\Lambda}^{12} \mathrm{~B}$ hypernuclear states were deduced with $\mathrm{a} \pm 1 \mathrm{MeV}$ uncertainty in the binding energy, $B_{\Lambda}$ (g.s.) $=11.23 \pm 1.0 \mathrm{MeV}$. As we discussed in the first chapter, the mesonic hypernuclear production mechanism does not allow obtaining a high resolution spectrum because of the quality of the secondary mesonic beams.

In our experiment, as expected, an energy resolution in of a few hundred $\mathrm{keV}$ was obtained. The values presented in Table 5.2.4 set an energy resolution record in reaction 
spectroscopy. Until now, the best energy resolution that has been available in reaction spectroscopy was reported to be around $670 \mathrm{keV}$ by the recent experiment E94-107 conducted in Hall A at Jefferson Lab [80] (Fig. 5.2.3). The strength and location of the peaks is consistent with our spectroscopy. It is also similar to the spectrum obtained in E89-009 (HNSS) experiment (Fig. 1.8.2).

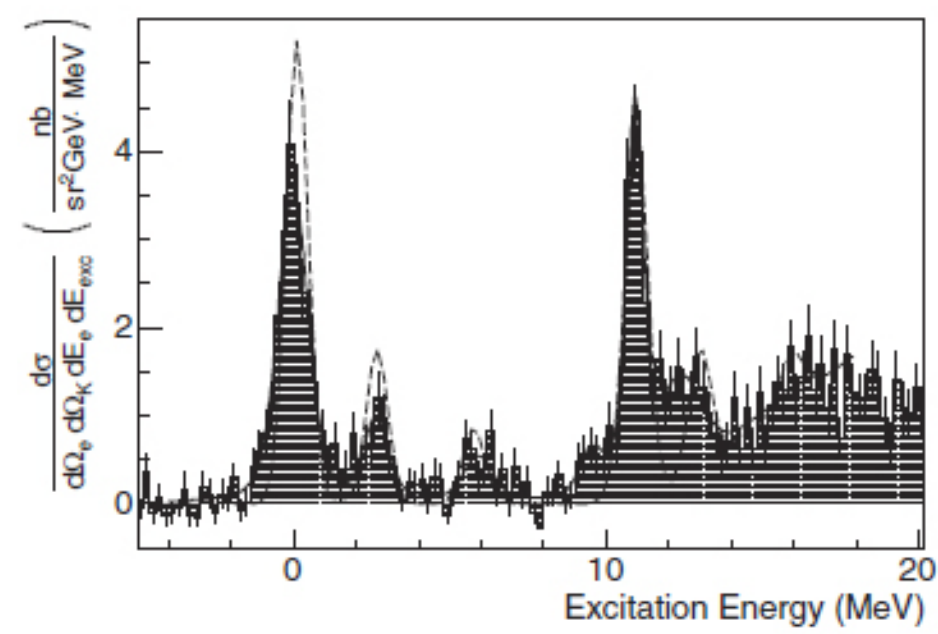

Figure 5.2.3 The ${ }_{\Lambda}^{12} \mathrm{~B}$ excitation energy spectrum obtained in E94-107 experiment in Hall A at JLab [80].

Although the sub-MeV resolution in our experiment sets a record in hypernuclear spectroscopy, we do not have sufficient statistics of energy resolution to recognize the fine structure of the states. The intrinsic width of the states is on the order of $100 \mathrm{keV}$ and the relative separation of the coupled states is predicted to be around $150 \mathrm{keV}$ (see Table 5.2.3). It is worth mentioning, however, that the HKS experiment did not have the ambition of resolving such closely positioned states and primarily aimed to measure the hypernuclear states with a resolution between $350-450 \mathrm{keV}$. As we can see from Table 5.2.4, the expected resolution has been successfully achieved. 
A comparison of the ${ }_{\Lambda}^{12} \mathrm{~B}$ spectroscopy results from the current study with results obtained in E89-009 (Hall C) and E94-107 (Hall A) is presented in Table 5.2.5. The uncertainties shown in the table are combined from statistical and systematic uncertainties. The binding energy measured by the HNSS experiment has been reported as $B_{\Lambda}=11.52 \pm 0.35 \mathrm{MeV}$ [84]. That is in agreement with the binding energy extracted in our study, $B_{\Lambda}=11.390 \pm 0.11 \mathrm{MeV}$ and is also in good agreement with the previously accepted value of $B_{\Lambda}=11.37 \pm 0.06 \mathrm{MeV}$, which comes from emulsion data for the $\pi^{-}+$ $3 \alpha$ decay. A comparison of the experimental excitation energies with theoretical predictions is plotted in Fig. 5.2.4. As we observe, the energy values are consistent within the error bars, except of the peak \#3. Good agreement with the theoretical predictions suggests the validity of the theoretical framework [77] used in calculations, however the core-excited $\left(2^{-}, 1^{-}\right)$doublet state that correspond to peak \#3 produces some level of disagreement.

Table 5.2.5 Comparison of the ${ }_{\Lambda}^{12} \mathrm{~B}$ spectroscopy obtained by E89-099 in Hall C, E94-107 in Hall A and E01-011 (current study) experiments.

\begin{tabular}{|c|c|c|c|c|c|c|c|}
\hline Peaks & \multicolumn{2}{|c|}{$\# 1$ g.s., $J^{p}=\left(1^{-}, 2^{-}\right)$} & \multicolumn{2}{|c|}{$\# 2$ c.e. $. J^{p}=\left(0^{-}, 1^{-}\right)$} & $\# 3$ c.e., $J^{p}=\left(2^{-}, 1^{-}\right)$ & \multicolumn{2}{|c|}{$\# 4$ g.S., $J^{p}=\left(2^{+}, 3^{+}\right)$} \\
\hline Struc & ure ${ }^{11} \mathrm{~B}(3 / 2$ & ;g.s. $) \otimes s_{1 / 2 \Lambda}$ & ${ }^{11} \mathrm{~B}\left(3 / 2^{-} ; 2.12\right)$ & $\otimes s_{1 / 2 \Lambda}$ & $\left./ 2^{-} ; 5.02\right) \otimes s_{1 / 2 \Lambda}$ & ${ }^{11} \mathrm{~B}\left(3 / 2^{-} ; 2.12\right.$ & $2) \otimes p_{1 / 2 \Lambda} \mid \otimes p_{3 / 2 \Lambda}$ \\
\hline \multirow[b]{2}{*}{ है } & \multirow{2}{*}{$\begin{array}{l}\text { Theoretical } \\
E_{x}, \mathrm{MeV}\end{array}$} & \multicolumn{2}{|c|}{ E89-009 (HNSS), Hall C } & \multicolumn{2}{|c|}{ E94-107, Hall A } & \multicolumn{2}{|c|}{ E01-011, Hall C } \\
\hline & & $E_{x}, \mathrm{MeV}$ & FWHM, MeV & $E_{x}, \mathrm{MeV}$ & FWHM, MeV & $E_{x}, \mathrm{MeV}$ & FWHM, MeV \\
\hline$\# 1$ & 0.0 & 0.0 & 0.92 & $0.0 \pm 0.03$ & 1.1 & $0.0 \pm 0.07$ & 0.4 \\
\hline$\# 2$ & 2.670 & $2.5 \pm 0.36$ & 0.92 & $2.65 \pm 0.10$ & 0.95 & $2.62 \pm 0.08$ & 0.39 \\
\hline$\# 3$ & 5.743 & $5.4 \pm 0.42$ & 0.92 & $5.92 \pm 0.13$ & 1.13 & $6.20 \pm 0.09$ & $0.54 \pm 0.13$ \\
\hline$\# 4$ & 11.05 & $11.0 \pm 0.32$ & 0.92 & $10.93 \pm 0.03$ & $0.67 \pm 0.15$ & $11.11 \pm 0.09$ & $0.42 \pm 0.12$ \\
\hline
\end{tabular}




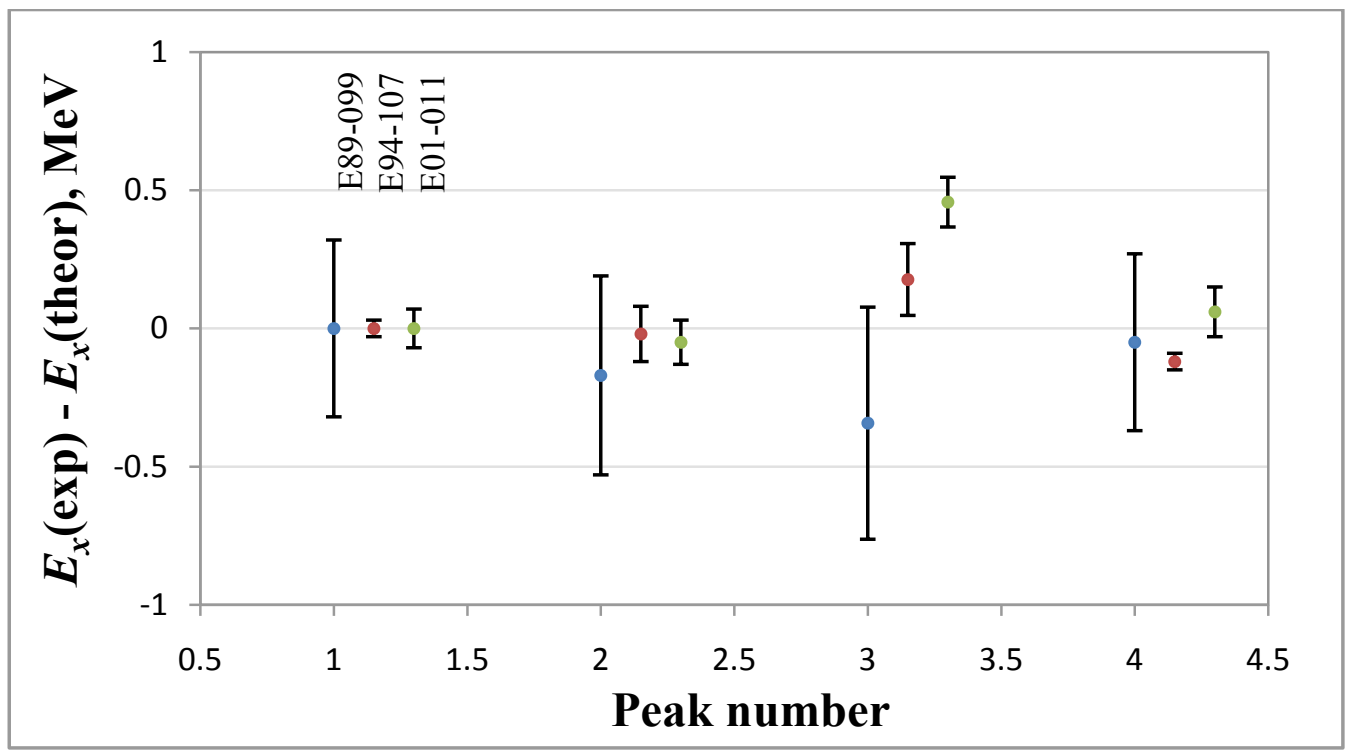

Figure 5.2.4 Visual comparison of the experimental excitation energy from its theoretically predicted value. The horizontal axis represents the number of the peak (according to assignment in Table 5.2.3). The peak numbers were shifted with respect to HNSS by 0.15 and 0.3 for E94-107 and E01-011, respectively.

\section{Comparison with the mirror hypernuclei}

To compare the current ${ }_{\Lambda}^{12} \mathrm{~B}$ spectrum with its mirror hypernuclei we look at the ${ }_{\Lambda}^{12} \mathrm{C}$ spectrum obtained by the meson-production reaction. Figure 5.2.5 displays the ${ }_{\Lambda}^{12} \mathrm{C}$ hypernuclear spectrum produced at KEK [38] and DAФNE [85] accelerator facilities. In the E369 experiment at KEK a secondary $\pi^{-}$beam was used, while FINUDA at DAФNE utilized slow negative kaons from $\varphi(1020)$ decay, stopping them in thin nuclear targets. Both spectra have very similar peaks positioned at approximately the same distances. In its ground state the ${ }_{\Lambda}^{12} \mathrm{C}$ hypernucleus has a $p$-shell neutron-hole configuration, almost identical to the ${ }_{\Lambda}^{12} \mathrm{~B} p$-shell proton-hole structure. The two most prominent peaks $\# 1$ and 
$\# 5$ (KEK, left), and \#1 and \#6 (FINUDA, right) correspond to coupling of the ${ }^{11} \mathrm{C} 3 / 2^{-}$ ground state with a $\Lambda$ in the $1 / 2^{+} s$ orbital and mixture of $3 / 2^{-}$and $1 / 2^{-} \Lambda p$ states, respectively. The core-excited states located in between the two large peaks are present on the spectra as well. Both data were fit with Gaussians - six for the left spectrum and seven for the right. The results of the fits are shown in Table 5.2.6.
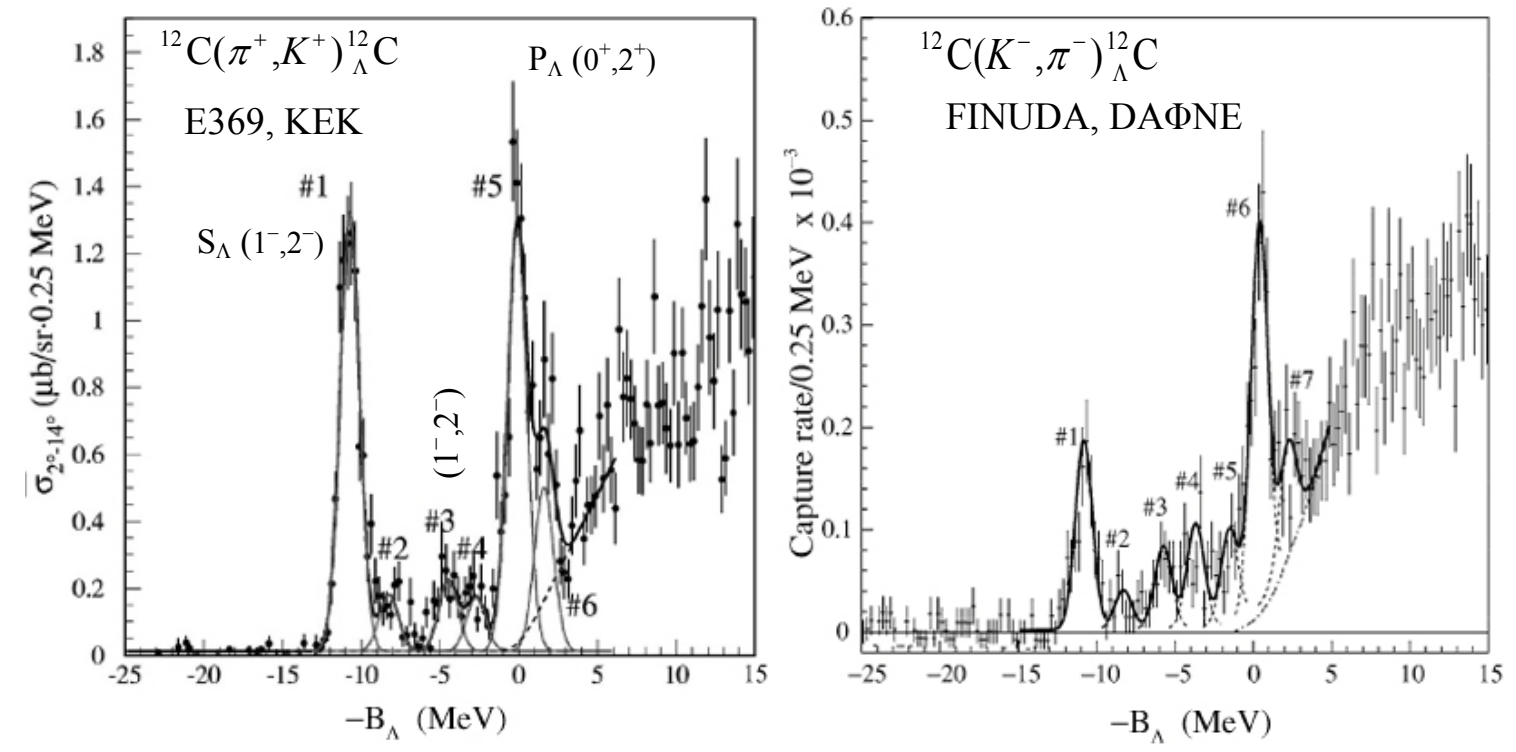

Figure 5.2.5 The ${ }_{\Lambda}^{12} \mathrm{C}$ spectroscopy obtained in E369 experiment at KEK via $\left(\pi^{+}, K^{\dagger}\right)$ reaction (left) [38] and in FINUDA experiment at DAФNE via $\left(K^{\prime}, \pi^{-}\right)$reaction (right) [85].

Table 5.2.6 Fitting results for ${ }_{\Lambda}^{12} \mathrm{C}$ spectra obtained in FINUDA at DAФNE [85] and E369 at KEK [38].

\begin{tabular}{|c|c|c|c|c|c|c|c|}
\hline \multirow{2}{*}{ 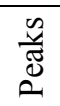 } & \multicolumn{3}{|c|}{ FINUDA at DA $\Phi$ NE } & \multicolumn{3}{|c|}{ E369 at KEK } & Theor., [86] \\
\hline & & $B_{\Lambda}, \mathrm{MeV}$ & $E_{x}, \mathrm{MeV}$ & & $B_{\Lambda}, \mathrm{MeV}$ & $E_{x}, \mathrm{MeV}$ & $E_{x}, \mathrm{MeV}$ \\
\hline$\# 1$ & g.s. $\otimes s_{\Lambda}$ & $10.94 \pm 0.06$ & 0 & g.s. $\otimes s_{\Lambda}$ & 10.76 & 0 & 0 \\
\hline$\# 2$ & ${ }^{11} \mathrm{C}\left(1 / 2^{-}\right) \otimes s_{\Lambda}$ & $8.4 \pm 0.2$ & $2.54 \pm 0.2$ & ${ }^{11} \mathrm{C}\left(1 / 2^{-}\right) \otimes s_{\Lambda}$ & $8.25 \pm 0.17$ & $2.51 \pm 0.17$ & 1.75 \\
\hline$\# 3$ & ${ }^{11} \mathrm{C}\left(3 / 2^{-}\right) \otimes s_{\Lambda}$ & $5.9 \pm 0.1$ & $5.04 \pm 0.1$ & ${ }^{11} \mathrm{C}\left(3 / 2^{-}\right) \otimes s_{\Lambda}$ & $4.46 \pm 0.11$ & $6.30 \pm 0.11$ & 4.90 \\
\hline$\# 4$ & & $3.8 \pm 0.1$ & $7.14 \pm 0.1$ & & $2.70 \pm 0.19$ & $8.06 \pm 0.19$ & \\
\hline$\# 5$ & & $1.6 \pm 0.2$ & $9.34 \pm 0.2$ & g.s. $\otimes p_{\Lambda}$ & $0.10 \pm 0.04$ & $10.66 \pm 0.04$ & \\
\hline$\# 6$ & g.s. $\otimes p_{\Lambda}$ & $-0.27 \pm 0.06$ & $11.21 \pm 0.06$ & & $-1.61 \pm 0.09$ & $12.37 \pm 0.09$ & 10.60 \\
\hline$\# 7$ & & $-2.1 \pm 0.2$ & $13.04 \pm 0.2$ & & & & \\
\hline
\end{tabular}


As stated in the table's references, the peaks $\# 2$ and \#3 in both data are composed of the ${ }^{11} \mathrm{C}\left(1 / 2^{-} ; 2.00\right) \otimes s_{\Lambda}$ and ${ }^{11} \mathrm{C}\left(3 / 2^{-} ; 4.8\right) \otimes s_{\Lambda}$ core-excited states, respectively. In the paper from the FINUDA experiment, the peaks $\# 4$ and $\# 5$ are referred to as being not well understood. According to the level scheme of the ${ }^{11} \mathrm{C}$ core nucleus, the $3 / 2^{+}$state with an excitation energy of $7.5 \mathrm{MeV}$ could be one of the positive parity excited states to explain peak \#4 in both experiments. Since this explanation is not confirmed in the literature, we will concentrate on the first two core-excited states. Table 5.2.7 contains the excitation energies of the first $1 / 2^{-}$and second $3 / 2^{-}$excited states for boron and carbon nuclei and hypernuclei.

Table 5.2.7 Excitation energies of the first two excited states of carbon and boron.

\begin{tabular}{ccc|cc}
\hline $\begin{array}{c}{ }^{11} \mathrm{C} \text { core, } \\
\left(\text { state } ; E_{\mathrm{x}}, \mathrm{MeV}\right.\end{array}$ & ${ }^{12} \mathrm{C}$ hypernucleus, (state; $\left.E_{\mathrm{x}}, \mathrm{MeV}\right)$ & $\begin{array}{c}{ }^{11} \mathrm{~B} \text { core, } \\
\left(\text { state; } E_{\mathrm{x}}, \mathrm{MeV}\right)\end{array}$ & $\begin{array}{c}{ }_{\Lambda}^{12} \mathrm{~B} \text { hypernucleus, } \\
\left.\text { (state; } E_{\mathrm{x}}, \mathrm{MeV}\right) \\
\text { HKS }\end{array}$ \\
\hline$\left(1 / 2^{-}\right) ; 2.0$ & FINUDA & KEK & & $\left(0^{-}, 1^{-}\right) ; 2.54 \pm 0.2$ \\
\hline$\left(3 / 2^{-}\right) ; 4.8$ & $\left(1^{-}, 2^{-}\right) ; 5.04 \pm 0.1$ & $\left(0^{-}, 1^{-}\right) ; 2.51 \pm 0.17$ & $\left(1 / 2^{-}\right) ; 2.12$ & $\left(0^{-}, 1^{-}\right) ; 2.62 \pm 0.07$ \\
\hline
\end{tabular}

As we see from the table, the energy needed to excite the core nucleus increases when a $\Lambda$ hyperon is embedded inside the core, indicating that the core itself becomes more strongly bound. That implies that the strong $\Lambda \mathrm{N}$ interaction provides a binding effect that causes the nucleons to become bound stronger in the nucleus than they initially were without presence of the $\Lambda$. The changes in the excitation energy are summarized in Table 5.2.8 and plotted in Fig. 5.2.6. An approximate $0.5 \mathrm{MeV}$ change in energy for the first $1 / 2^{-}$excited state is observed for both FINUDA and KEK experiments in ${ }_{\Lambda}^{12} \mathrm{C}$ data and HKS experiment in ${ }_{\Lambda}^{12} \mathrm{~B}$ data. In the case of the second $3 / 2^{-}$excited state, the shift in 
excitation energy is different in FINUDA and KEK experiments. The result from the HKS experiment on a boron target is about $1.2 \mathrm{MeV}$, which is close to KEK's value of 1.5 MeV. The large difference in the $3 / 2^{-}$configuration between FINUDA and KEK is obvious. It is the result of a different approach to fitting the peaks. In the FINUDA analysis the fit was applied for four core-excited states between $\Lambda s$ and $\Lambda p$ peaks, while in KEK only three core-excited states were analyzed. That created a certain ambiguity in getting the mean value of the $3 / 2^{-}$excited state. In the HKS data we observe only two core-excited states with significant strength. If we assume symmetry between the $\Lambda p$ and $\Lambda n$ interactions (charge symmetry), then the HKS and KEK (or FINUDA) points have to be in perfect agreement.

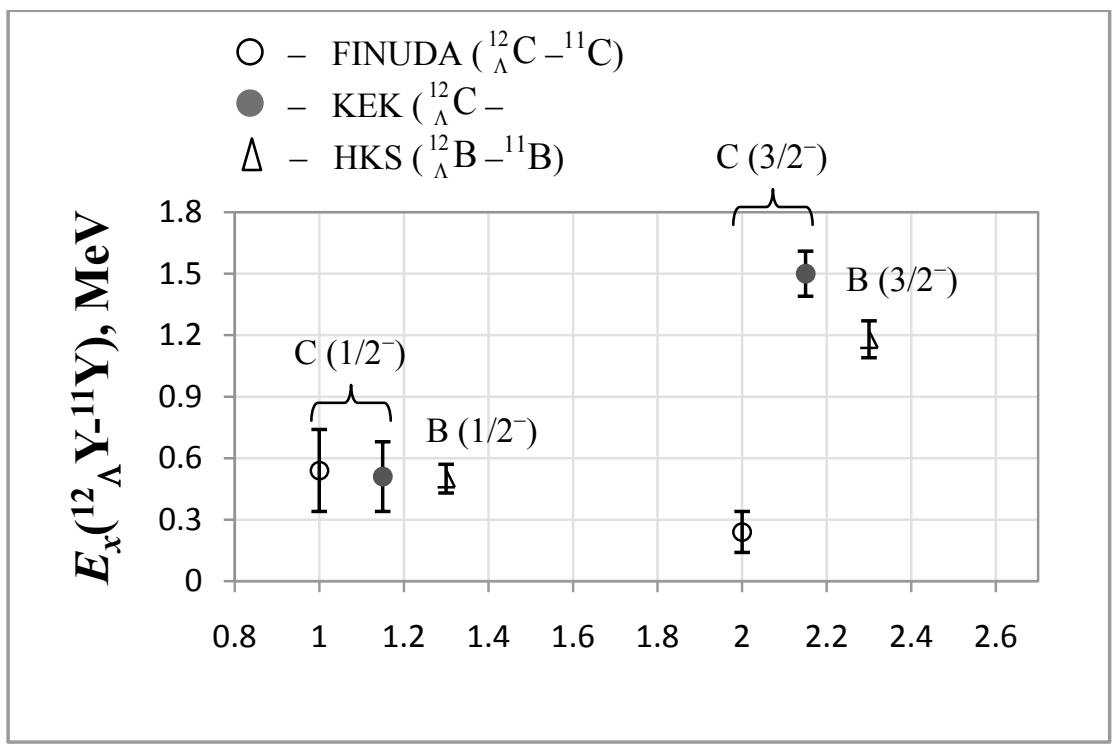

Figure 5.2.6 The change in excitation energy of the $1 / 2^{-}$and $3 / 2^{-}$excited states when embedding a $\Lambda$ into the two mirror boron and carbon core nuclei. 
Table 5.2.8 The change in excitation energy of $1 / 2^{-}$and $3 / 2^{-}$excited states.

\begin{tabular}{c|cc|c}
\hline \multirow{2}{*}{ state $\left(E_{x}\right)$} & $E_{x}\left({ }_{\Lambda}^{12} \mathrm{C}(\right.$ ex.s - g.s $)-{ }^{11} \mathrm{C}($ ex.s. - g.s. $\left.)\right), \mathrm{MeV}$ & $E_{x}\left({ }_{\Lambda}^{12} \mathrm{~B}(\right.$ ex.s. - g.s $)-{ }^{11} \mathrm{~B}($ ex.s. -g.s. $\left.)\right), \mathrm{MeV}$ \\
\cline { 2 - 4 } & FINUDA & KEK & HKS \\
\hline $1 / 2^{-}(2.00)$ & $0.54 \pm 0.2$ & $0.51 \pm 0.17$ & $0.50 \pm 0.07$ \\
\hline $3 / 2^{-}(4.80)$ & $0.24 \pm 0.2$ & $1.50 \pm 0.11$ & $1.18 \pm 0.09$ \\
\hline
\end{tabular}

\subsection{Spectroscopy of ${ }_{\Lambda}^{7} \mathrm{He}$ hypernuclei}

\section{$A=7$ isospin-triplet}

The ${ }_{\Lambda}^{7} \mathrm{He}$ hypernucleus belongs to the $I=1$ isospin-triplet that also includes two other $A=7$ hypernuclei: ${ }_{\Lambda}^{7} \mathrm{Li}$ and ${ }_{\Lambda}^{7} \mathrm{Be}$. The theoretical calculations of the excitation or binding energies of the hypernuclear states involve the solution of a many-body problem. The straightforward approach to the calculation of the seven-body nuclear system is extremely complicated and the cluster approach is used instead. The cluster model reduces the number of interacting bodies by combining some of the nucleons into stable clusters. One of the relatively recent cluster models for the $A=7$ iso-triplet, developed in the mid 90's, includes ${ }_{\Lambda}^{5} \mathrm{He}+\mathrm{N}+\mathrm{N}$ three-body calculations [87]. In such a model the $A=6$ nuclei $\left({ }^{6} \mathrm{He},{ }^{6} \mathrm{Li},{ }^{6} \mathrm{Be}\right)$ are investigated first and followed by the injection of the $\Lambda$ particle. Since the core $A=6$ nuclei utilize the $\alpha+\mathrm{N}+\mathrm{N}$ model, the same three-body formalism carries over to the $A=7$ hypernuclear system. Lately, a four-body cluster model for the $A=7$ hypernuclei has been developed [88]. It uses a $\alpha+\mathrm{N}+\mathrm{N}+\Lambda$ cluster configuration to describe these hypernuclei. In such a representation, the ${ }_{\Lambda}^{7} \mathrm{He}$ becomes $\alpha+n+n+\Lambda$, while 
${ }_{\Lambda}^{7} \mathrm{Li}$ and ${ }_{\Lambda}^{7} \mathrm{Be}$ are composed of $\alpha+n+p+\Lambda$ and $\alpha+p+p+\Lambda$, respectively. Unlike the three-body model, which uses only the even-state $\Lambda \mathrm{N}$ interactions, the four-body model employs both even and odd-state $\Lambda \mathrm{N}$ interactions together with symmetric and antisymmetric spin-orbit couplings in the $\Lambda \mathrm{N}$ interaction potential. The $\Lambda \mathrm{N}-\Sigma \mathrm{N}$ coupling that represents a three-body interaction effect is also taken into consideration. The calculations employed the Nijmegen model NSC97f, where the contribution from the $\Sigma \mathrm{N}$ channel was renormalized into the $\Lambda \mathrm{N}$ interaction [88].

One of the interesting features of the $A=7$ iso-triplet that recently has been actively discussed by theoreticians is the possibility of observing charge symmetry breaking (CSB). Historically, the most reliable evidence of CSB comes from the $A=4$, $I=1 / 2$ hypernuclear multiplet: ${ }_{\Lambda}^{4} \mathrm{He}$ and ${ }_{\Lambda}^{4} \mathrm{H}$. The $\Lambda$ particle embedded inside of the ${ }^{3} \mathrm{He}$ nuclear core couples with the neutron, while within the ${ }^{3} \mathrm{H}$ nucleus it couples to a proton. Although the $\Lambda$ interacts with all nucleons, the fraction of interactions with $n$ and $p$ differs. The CSB effect, defined as $\mathrm{CSB}=B_{\Lambda}\left({ }_{\Lambda}^{4} \mathrm{He}\right)-B_{\Lambda}\left({ }_{\Lambda}^{4} \mathrm{H}\right)$, is not a zero value as would be expected. According to theoretical calculations it is $-0.05 \mathrm{MeV}$ for the $0^{+}$ground and $0.07 \mathrm{MeV}$ for the first $1^{+}$excited states [89]. Experimentally it was found to be $0.35 \pm 0.06$ $\mathrm{MeV}$ and $0.24 \pm 0.06 \mathrm{MeV}$, respectively [88]. The results of the four-body theoretical calculations that will be used in comparison to our ${ }_{\Lambda}^{7} \mathrm{He}$ experimental data will be with and without the CSB effect.

The spectroscopy for the $A=7$ triplet was attempted in the early 1970 's via $\pi^{-}$ mesonic decay of hypernuclei produced by stopped $K^{-}$in a nuclear emulsion [90]. The 
binding energy values averaged over all possible pion-decay modes were found for ${ }_{\Lambda}^{7} \mathrm{Li}$ and ${ }_{\Lambda}^{7}$ Be hypernuclei. Data for ${ }_{\Lambda}^{7} \mathrm{He}$ hypernuclei were available, however, low statistics together with background issues led to inconclusive results. The ${ }_{\Lambda}^{7} \mathrm{Li}$ hypernuclear spectrum was further improved by $\left(\pi^{+}, K^{+}\right)$reaction spectroscopy at KEK [91]. Our experiment provides the first high resolution spectrum of the missing ${ }_{\Lambda}^{7} \mathrm{He}$ hypernucleus, by that completing the hypernuclear spectroscopy of the $A=7$ iso-triplet.

The shell structure of the ${ }_{1}^{7} \mathrm{He}$ hypernucleus is created by the coupling of an $s$ shell $\Lambda$ to the $0^{+}$ground state and $2^{+}$excited state of ${ }^{6} \mathrm{He}$. The $\Lambda$ coupling to the ground state produces a $1 / 2^{+}$state, and $3 / 2^{+}$and $5 / 2^{+}$states when coupled to the first excited state. The theoretically predicted four-body cluster-model binding energies for each of the states are shown in Table 5.3.1 [88]. The table also contains the peak strengths in terms of the cross sections that have been estimated with the help of the elementary hypernuclear production process [92].

Table 5.3.1 Hypernuclear states of ${ }_{\Lambda}^{7} \mathrm{He}$ and their theoretically predicted binging energies [88] and cross sections [92].

\begin{tabular}{ccccccc}
\hline $\begin{array}{c}{ }^{6} \mathrm{He}, J^{p} \\
\text { (shell) }\end{array}$ & $\begin{array}{c}\Lambda, J^{p} \\
(\text { shell })\end{array}$ & ${ }_{\Lambda}^{7} \mathrm{He}, J^{p}$ & $\begin{array}{c}\text { Theor. binding } \\
\text { energy, }-B_{\Lambda}(\mathrm{MeV})\end{array}$ & \multicolumn{3}{c}{ Sheor. cross sections (nb/sr), } \\
\hline $0^{+}(p$, g.s.) & $1 / 2^{+}(s)$ & $1 / 2^{+}$ & -5.36 & 13.2 & 16.2 & KMAID \\
\hline \multirow{2}{*}{$2^{+}(p$, ex.1) } & \multirow{2}{*}{$1 / 2^{+}(s)$} & $3 / 2^{+}$ & -3.70 & 3.0 & 3.7 & 9.7 \\
\hline
\end{tabular}




\section{Spectroscopy}

The spectroscopy of the ${ }_{\Lambda}^{7} \mathrm{He}$ hypernuclei from this experiment is shown in Fig. 5.3.1. The statistics of the spectrum is relatively high, however, a significant part of it belongs to the accidental background shown by the blue hatched area. The ground state $\Lambda_{S}\left(1 / 2^{+}\right)$ is clearly seen on the spectrum between -5 and $-6 \mathrm{MeV}$. There also is some indication of the possible core-excited states on the right side of the peak, around $-4 \mathrm{MeV}$. Both bound and unbound regions contain somewhat large fluctuations equally distributed along the spectrum. The presence of such large statistical fluctuations makes firm statements about a state at $-4 \mathrm{MeV}$ questionable.

The fitting of the spectrum was performed in two steps. In the first step the ground state peak was fit with a Gaussian function and the rest of the data with a linear background and a square root in the quasi-free region. The fitting result from the first step is shown in Fig. 5.3.1. by the red line. The binding energy extracted from the fit is $B_{\Lambda}=$ $5.73 \pm 0.04 \mathrm{MeV}$ and the FWHM energy width is $\Delta B_{\Lambda}=466 \pm 80 \mathrm{keV}$. In the second step we will include in the fit the core-excited peak with the same energy resolution represented by a Gaussian. The result of this fit is shown in Fig. 5.3.2. 


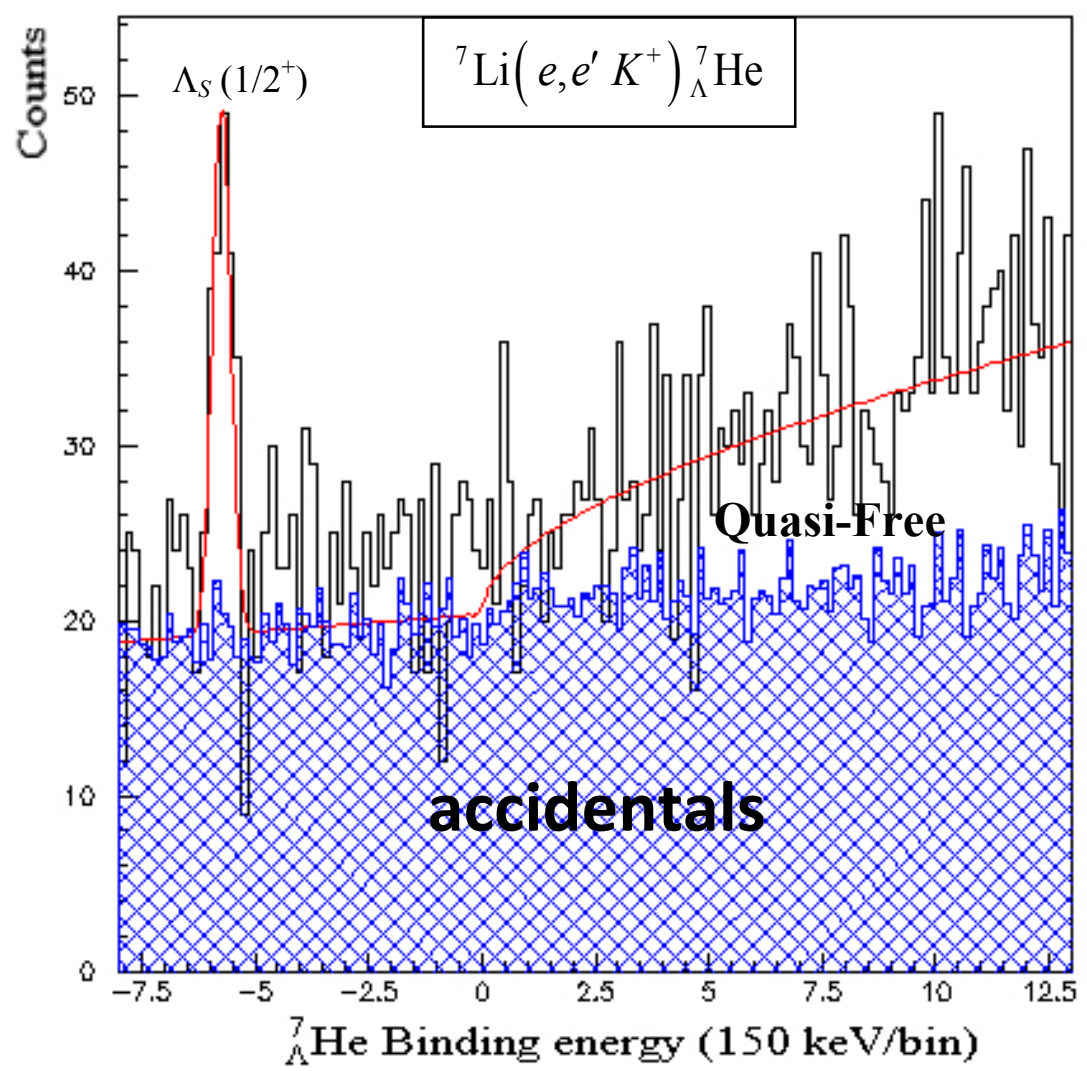

Figure 5.3.1 Binding energy spectrum $\left(-B_{\Lambda}\right)$ of ${ }_{\Lambda}^{7} \mathrm{He}$ hypernucleus (without core-excited peak).

The ratio of the strength of the ground state peak to the core-excited doublet is $4.12 \pm 2.23$. From Table 5.3.1 the ratio of the predicted cross sections of ground to coreexcited states ranges between 3.87 and 6.47. Even though the experimental ratio is within the predicted range, it is not sufficiently significant to provide a firm statement on the presence of the core-excited states. The second peak included in the fit does not change the mean value of the ground state and the FWHM energy resolution essentially is the same, $\Delta B_{\Lambda}=464 \pm 71 \mathrm{keV}$. The comparison of the results with theoretically predicted values is summarized in Table 5.3.2. 


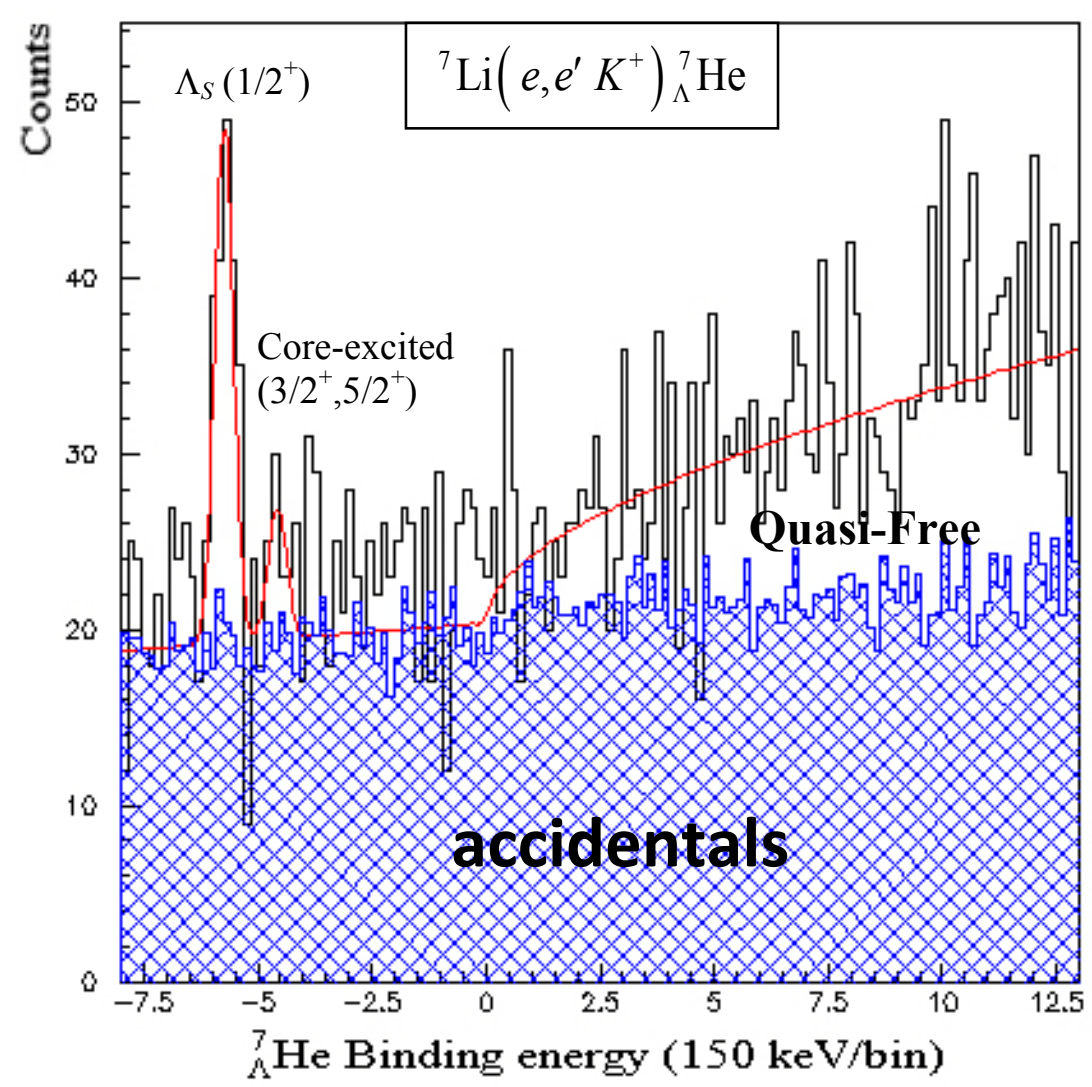

Figure 5.3.2 Binding energy spectrum $\left(-B_{\Lambda}\right)$ of ${ }_{\Lambda}^{7} \mathrm{He}$ hypernucleus (with core-excited peak).

Table 5.3.2 Comparison of experimental results to theoretical predictions for ${ }_{\Lambda}^{7} \mathrm{He}$ hypernuclei.

\begin{tabular}{cccccc}
\hline $\begin{array}{c}{ }^{6} \mathrm{He}, J^{p} \\
(\text { shell })\end{array}$ & $\begin{array}{c}\Lambda, J^{p} \\
(\text { shell })\end{array}$ & ${ }_{\Lambda}^{7} \mathrm{He}, J^{p}$ & $\begin{array}{c}\text { Theor. binding } \\
\text { energy, }-B_{\Lambda}(\mathrm{MeV})\end{array}$ & $\begin{array}{c}\text { Exp. binding } \\
\text { energy, }-B_{\Lambda}(\mathrm{MeV})\end{array}$ & $\begin{array}{c}\text { Energy width } \Delta B_{\Lambda} \\
(\mathrm{keV})\end{array}$ \\
\hline $0^{+}(p$, g.s. $)$ & $1 / 2^{+}(s)$ & $1 / 2^{+}$ & -5.36 & $\begin{array}{c}-5.730 \pm 0.041(\mathrm{st}) \\
\pm 0.11(\mathrm{sys})\end{array}$ & $464.15 \pm 71.43(\mathrm{st})$ \\
\hline $2^{+}(p$, ex.1) & $1 / 2^{+}(s)$ & $\begin{array}{c}3 / 2^{+} \\
5 / 2^{+}\end{array}$ & $\begin{array}{c}-3.70 \\
-3.62\end{array}$ & $\begin{array}{c}-4.575 \pm 0.113(\mathrm{st}) \\
\pm 0.11(\mathrm{sys})\end{array}$ & 466.64 (fixed) \\
\hline
\end{tabular}

As we see, the experimental value for binding energy exceeds the prediction. That might advocate for the presence of the charge symmetry breaking. However, it was shown by the authors of the theoretical predictions in Table 5.3.2 that addition of the CSB effect into their calculations reduces the binding of the ground state to $-5.16 \mathrm{MeV}$ 
[88]. The experimental result suggests that the hypernuclear $1 / 2^{+}$ground state is bound deeper than expected.

The prediction of the core-excited doublet state in the bound region comes from the specifics of the nuclear structure of the ${ }^{6} \mathrm{He}$ core and the strong interaction effect that $\Lambda$ particle brings when embedded inside. According to a cluster model, ${ }^{6} \mathrm{He}$ is built from a strongly bound ${ }^{4} \mathrm{He}(\alpha)$ core positioned in the center and two neutrons orbiting on the surface of the nucleus. This is a so called "halo" configuration, where core cluster and nucleons are separated by relatively large distances. As a result of this configuration, both neutrons in ${ }^{6} \mathrm{He}$ are weakly bound to the $\alpha$ core. The level diagram of the ${ }^{6} \mathrm{He}$ and the ${ }_{\Lambda}^{7} \mathrm{He}$ hypernucleus calculated according to the four-body cluster model is shown in Fig. 5.3.3 (left). While adding the $\Lambda$ particle inside of the ${ }^{6} \mathrm{He}$ nucleus the energy levels change their position relative to the breakup threshold. On the right side of the Fig. 5.3.3, the density distribution of $\alpha, \Lambda$, and the two weakly bound valence neutrons is plotted. 


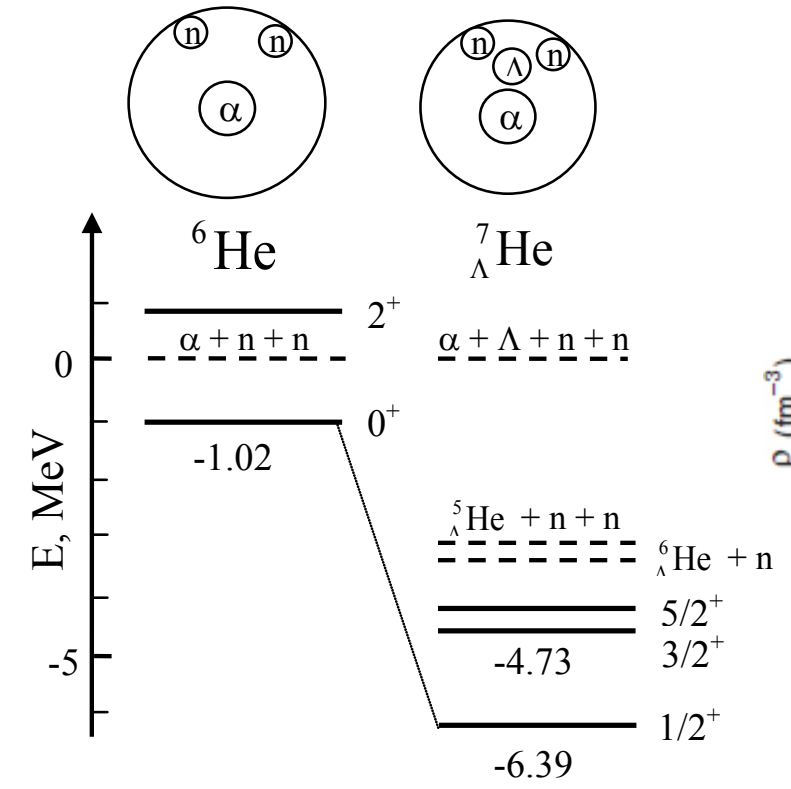

Levels diagram and structure for ${ }^{6} \mathrm{He}$ nucleus and ${ }_{\Lambda}^{7} \mathrm{He}$ hypernucleus

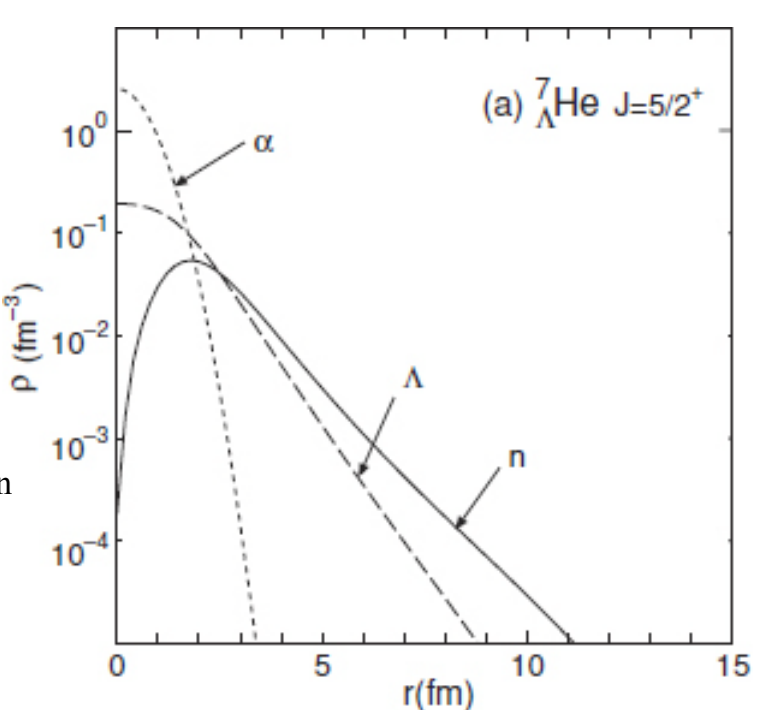

Density distribution of $\alpha, \Lambda$ and valence neutrons

Figure 5.3.3 Level diagrams for ${ }^{6} \mathrm{He}$ and ${ }_{\Lambda}^{7} \mathrm{He}$ calculated according to four-body cluster model [88] (left). The density distribution of the $\alpha, \Lambda$ and two neutrons inside of the ${ }_{\Lambda}^{7} \mathrm{He}$ hypernuclei [88] (right).

According to three-body ${ }^{6} \mathrm{He}$ cluster model the ground state level $0^{+}$is bound by $1.02 \mathrm{MeV}$ with respect to the $\alpha+n+n$ break up threshold. The first low-lying excited $2^{+}$ state is located in the unbound region $0.82 \mathrm{MeV}$ above threshold. When the $1 / 2^{+} \Lambda$ is embedded inside of the nucleus, it couples with the ${ }^{6} \mathrm{He}$ ground state resulting in a deeper bound, $B_{\Lambda} \approx 5.36 \mathrm{MeV}, 1 / 2^{+}$hypernuclear ground state. Coupling with the first excited state leads to the creation of a $\left(5 / 2^{+}, 3 / 2^{+}\right)$doublet that shifts into the bound region. That shift is caused by the interaction of the $\Lambda$ particle inside of the ${ }^{6} \mathrm{He}$ with the cluster components. According to the plot on the right in Fig. 5.3.3 the density distribution of the $\Lambda$ is strongly overlapped with the $\alpha$ core. As expected the valence neutrons spend most of 
their time in halo orbits. Despite its strong binding to the $\alpha$, the $\Lambda$ has a long tail that crosses the neutron distribution. The long $\Lambda$ tail means that it spends a significant amount of time in the space between the clusters. It is most probable that the appearance of the hypernuclear core-excited state doublet in the bound region is mostly because of the interaction of the $\Lambda$ with the valence neutrons. It cannot be because of the $\Lambda$ and $\alpha$ core interaction. The reason is that the first excited state is separated from the ground state by $\approx 1.8 \mathrm{MeV}$ in ${ }^{6} \mathrm{He}$ and by $\approx 20 \mathrm{MeV}$ in ${ }^{4} \mathrm{He}$. It suggests that the $2^{+}$excited state in ${ }^{6} \mathrm{He}$ is composed purely by rearrangement of the valence neutrons from the ground state configuration. Therefore the interaction of the embedded $\Lambda$ with the halo neutrons causes the excited state to move inside of the bound region, which in turn leads to a decrease in the size of the nucleus. This effect is the so called "glue effect" and is discussed, for example in [88].

\section{Comparison to ${ }_{A}^{7} \mathrm{Li}$ and ${ }_{\Lambda}^{7} \mathrm{Be} A=7$ iso-triplet members}

As we mentioned before, the pion decay emulsion data provided us with the measurement of ground states for both ${ }_{\Lambda}^{7} \mathrm{Li}$ and ${ }_{\Lambda}^{7} \mathrm{Be}$. The spectroscopy of ${ }_{\Lambda}^{7} \mathrm{Li}$ was later measured at KEK with a $\pi^{+}$beams and the energy levels were investigated by $\gamma$ ray spectroscopy at KEK and BNL. The results of emulsion experiments with $\pi^{-}$beams for the $A=7$ hypernuclei triplet are shown in Fig. 5.3.4. As we indicated on the figure, all of the members of $A=7$ triplet have a halo structure. Since in these hypernuclei, the embedded $\Lambda$ has a strong interaction with the valence nucleons, it is a very interesting case for 
studying the CSB effect. To understand this effect one has to look at the $\Lambda \mathrm{N}$ interaction in the halo area, but not in the $\alpha$ cluster. The effect of the $\Lambda$ on the core-excited states carries important information. Currently the high resolution spectroscopy is available only for ${ }_{1}^{7} \mathrm{Li}$ from KEK [91]. It is shown in Fig. 5.3.5. Table 5.3.3 lists the results of the fits.

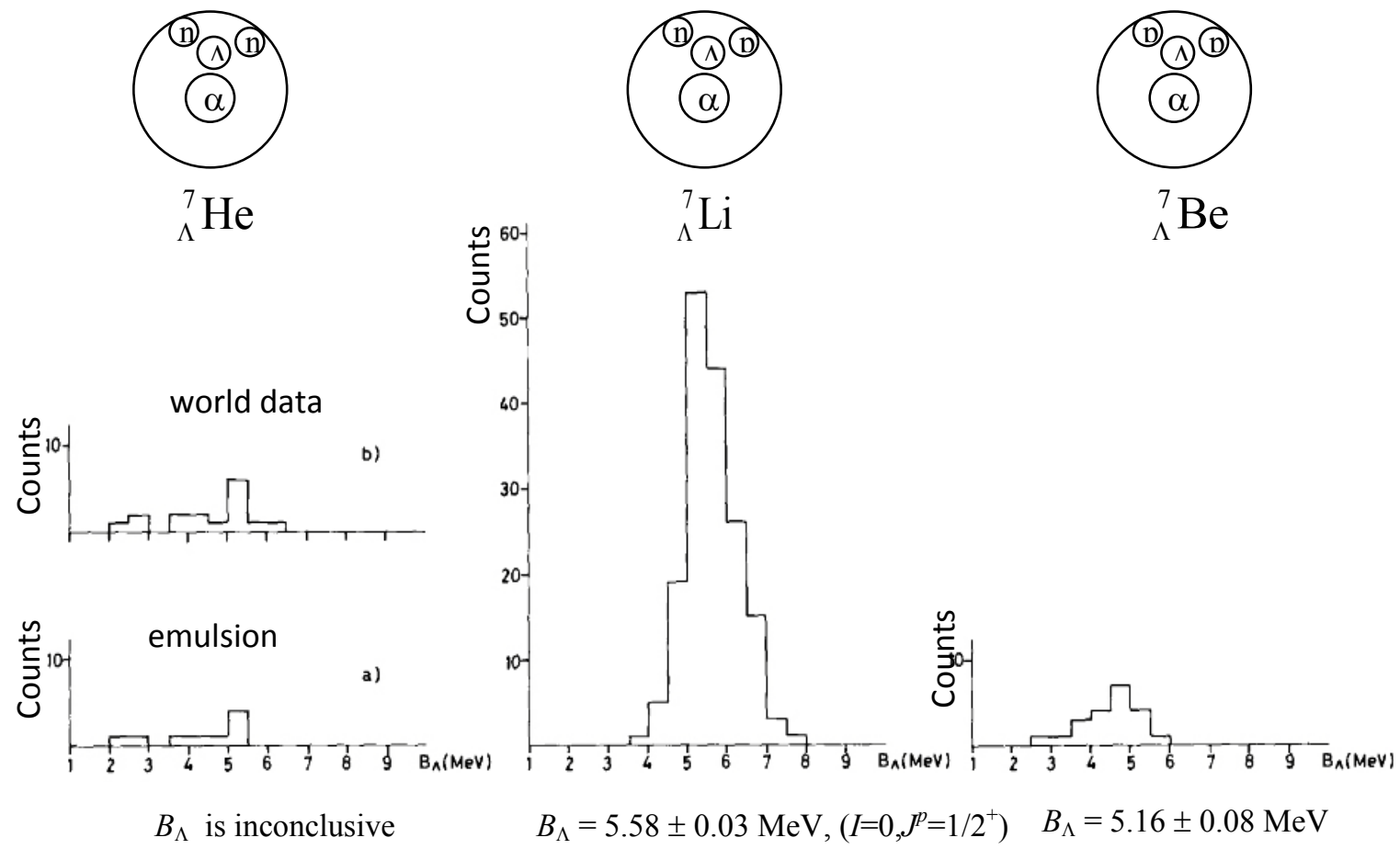

Figure 5.3.4 Results for $A=7$ iso-triplet from emulsion $\pi^{-}$decay experiments [90]. 


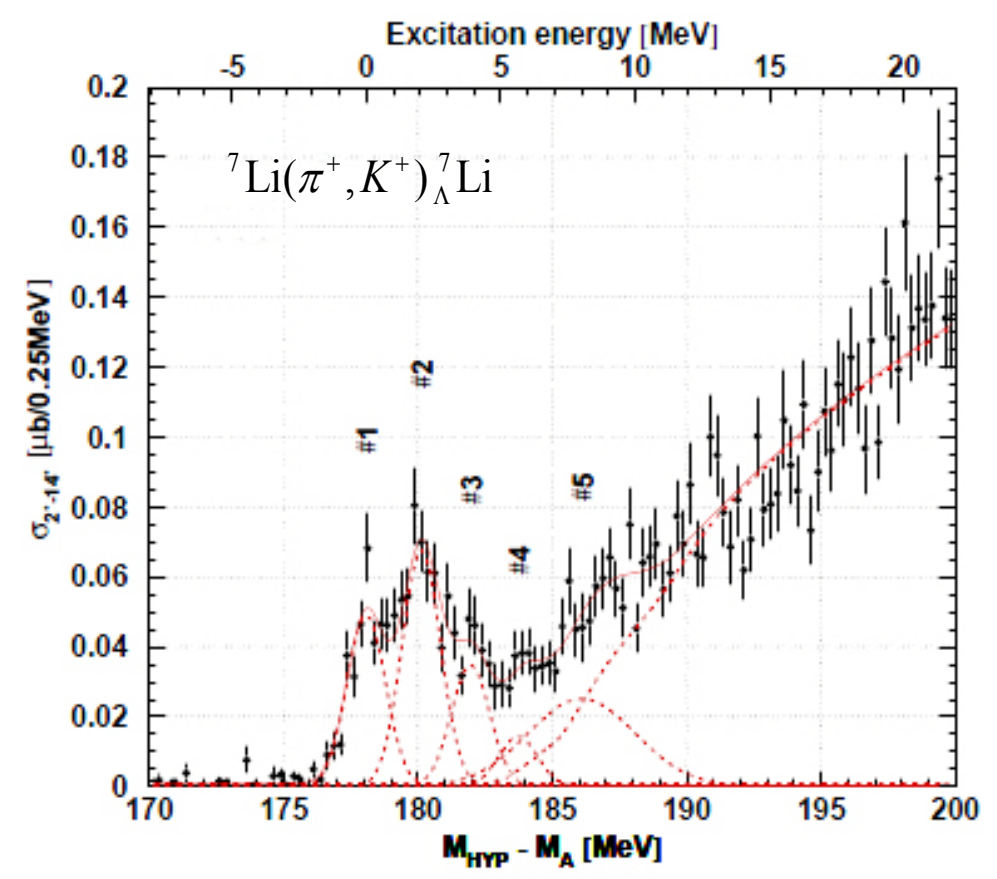

Figure 5.3.5 Hypernuclear spectrum of ${ }_{\Lambda}^{7} \mathrm{Li}$ measured by SKS at KEK in E336 experiment [91].

Table 5.3.3 Fitting results for ${ }_{\Lambda}^{7} \mathrm{Li}$ spectrum measured at KEK by E336 experiment [91].

\begin{tabular}{cccc}
\hline Peak \# & $J^{p}(I)$ & $B_{\Lambda}$ and $E_{x}, \mathrm{MeV}$ & FWHM, MeV \\
\hline$\# 1$ & $1 / 2^{+}(I=0)$ & $B_{\Lambda}=5.22 \pm 0.08$ & 1.81 (fixed) \\
& $3 / 2^{+}(I=0)$ & & 1.81 (fixed) \\
\hline \multirow{2}{*}{$\# 2$} & $5 / 2^{+}(I=0)$ & $E_{x}=2.05$ (fixed) & 1.81 (fixed) \\
\hline$\# 3$ & $7 / 2^{+}(I=0)$ & & 1.81 (fixed) \\
\hline \multirow{2}{*}{$\# 4$} & $1 / 2^{+}(I=1)$ & $E_{x}=3.88$ (fixed) & $3.81 \pm 0.81$ \\
\hline$\# 5$ & $5 / 2^{+}(I=1)$ & $E_{x}=5.61 \pm 0.24$ & \\
\hline
\end{tabular}

The energy for peaks \#2 and \#3 was determined in $\gamma$-ray spectroscopy and was held fixed when fitting the spectrum [93]. The FWHM energy resolution of the first four peaks was fixed to $1.81 \mathrm{MeV}$. This number was determined from the ${ }_{\Lambda}^{12} \mathrm{C}$ spectrum obtained during the experiment and included corrections for difference in the energy loss 
straggling in the ${ }_{\Lambda}^{7} \mathrm{Li}$ and ${ }_{\Lambda}^{12} \mathrm{C}$ targets. To compare ${ }_{\Lambda}^{7} \mathrm{He}$ and ${ }_{\Lambda}^{7} \mathrm{Li}$ we have to look at the same isospin $I=1$ states. That constrains us to consideration of only peaks \#3 and \#4 in the ${ }_{\Lambda}^{7} \mathrm{Li}$ spectrum, where we have a $1 / 2^{+}$singlet from ${ }^{6} \mathrm{Li}\left(0^{+} ; 3.56\right)$ and $s_{1 / 2 \Lambda}$ coupling and $\left(3 / 2^{+}, 5 / 2^{+}\right)$doublet created by ${ }^{6} \operatorname{Li}\left(2^{+} ; 4.31\right)$ and $s_{1 / 2 \Lambda}$ coupling. According to the results from Table 5.3.3, the separation between these states is $1.73 \pm 0.24 \mathrm{MeV}$. From our experimental ${ }_{1}^{7} \mathrm{He}$ data we find $1.16 \pm 0.11 \mathrm{MeV}$ separation between core-excited and ground states. The separations for the two cases are not consistent, however, they are on the same order. As one can see from the ${ }_{1}^{7} \mathrm{Li}$ spectrum, Fig. 5.3.5, the fitting results for peaks \#3 and \#4 are very tentative. Until the high resolution spectroscopy of ${ }_{A}^{7} \mathrm{Li}$ hypernucleus is obtained with FWHM resolution compatible to our results $(\approx 0.47 \mathrm{MeV})$, it is difficult to perform a reliable quantitative comparison.

In order to investigate the effect of a $\Lambda$ interaction with the nucleons of the core we look at the excitation energies of the first excited state with $I=1$ isospin in nuclear and hypernuclear systems of $\mathrm{He}$ and $\mathrm{Li}$, Table 5.3.4.

Table 5.3.4 Excitation energies of the $I=1$ ground and first excited states of lithium and helium.

\begin{tabular}{cc|cc}
\hline $\begin{array}{c}{ }^{6} \mathrm{Li} \text { core, } \\
(\text { state }) ; E_{\mathrm{x}}, \mathrm{MeV}\end{array}$ & $\begin{array}{c}{ }_{\Lambda}^{7} \mathrm{Li} \text { hypernucleus, } \\
\text { (state) } ; E_{\mathrm{x}}, \mathrm{MeV} \\
\mathrm{KEK}\end{array}$ & $\begin{array}{c}{ }^{6} \mathrm{He} \text { core, } \\
\text { (state) } ; E_{\mathrm{x}}, \mathrm{MeV}\end{array}$ & $\begin{array}{c}{ }_{\Lambda}^{7} \mathrm{He} \text { hypernucleus, } \\
\text { (state) } ; E_{\mathrm{x}}, \mathrm{MeV} \\
\text { HKS }\end{array}$ \\
\hline$\left(0^{+}\right) ; 3.56$ & $\left(1 / 2^{+}\right) ; 3.88$ & $\left(0^{+}\right) ; 0.00$ & $\left(1 / 2^{+}\right) ; 0.00$ \\
\hline$\left(2^{+}\right) ; 5.37$ & $\left(3 / 2^{+}, 5 / 2^{+}\right) ; 5.61 \pm 0.24$ & $\left(2^{+}\right) ; 1.80$ & $\left(3 / 2^{+}, 5 / 2^{+}\right) ; 1.16 \pm 0.14$ \\
\hline
\end{tabular}

We want to compare how the separation of the states $\left(2^{+}\right)-\left(0^{+}\right)$and $\left(3 / 2^{+}, 5 / 2^{+}\right)-\left(1 / 2^{+}\right)$ changes when the $\Lambda$ particle is introduced inside of the core. The results of the comparison are presented in Table 5.3.5 and the plot of the observed changes in 
excitation energy is shown in Fig. 5.3.6. In both cases a negative change is observed. It contradicts our results for the ${ }_{\Lambda}^{12} \mathrm{~B}$ hypernucleus, where a positive change in energy was found. According to our expectations, the $\Lambda$ 's binding effect has to be revealed in the helium hypernucleus as well, however, one has to notice the poor statistics in the coreexcited state region in both helium and lithium spectra. The fit of the core-excited states in ${ }_{\Lambda}^{7} \mathrm{Li}$ spectrum is very tentative, as well as the fit in ${ }_{\Lambda}^{7} \mathrm{He}$ spectrum obtained in our experiment. To clearly identify the binding effect that a $\Lambda$ introduces into these nuclei we have to obtain a high statistics and high resolution spectrum comparable with the ${ }_{\Lambda}^{12} \mathrm{~B}$ hypernuclear spectrum measured in this experiment.

Table 5.3.5 The change in excitation energy of the first excited and ground states of lithium and helium.

\begin{tabular}{|c|c}
\hline$E_{x}\left({ }_{\Lambda}^{7} \operatorname{Li}\left(\left(3 / 2^{+}, 5 / 2^{+}\right)-\left(1 / 2^{+}\right)\right)-{ }^{6} \operatorname{Li}\left(\left(2^{+}\right)-\left(0^{+}\right)\right)\right)$ & $E_{x}\left({ }_{\Lambda}^{7} \mathrm{He}\left(\left(3 / 2^{+}, 5 / 2^{+}\right)-\left(1 / 2^{+}\right)\right)-{ }^{6} \mathrm{He}\left(\left(2^{+}\right)-\left(0^{+}\right)\right)\right)$ \\
\hline $\mathrm{KEK}$ & HKS \\
\hline$-0.08 \pm 0.24 \mathrm{MeV}$ & $-0.65 \pm 0.14 \mathrm{MeV}$ \\
\hline
\end{tabular}

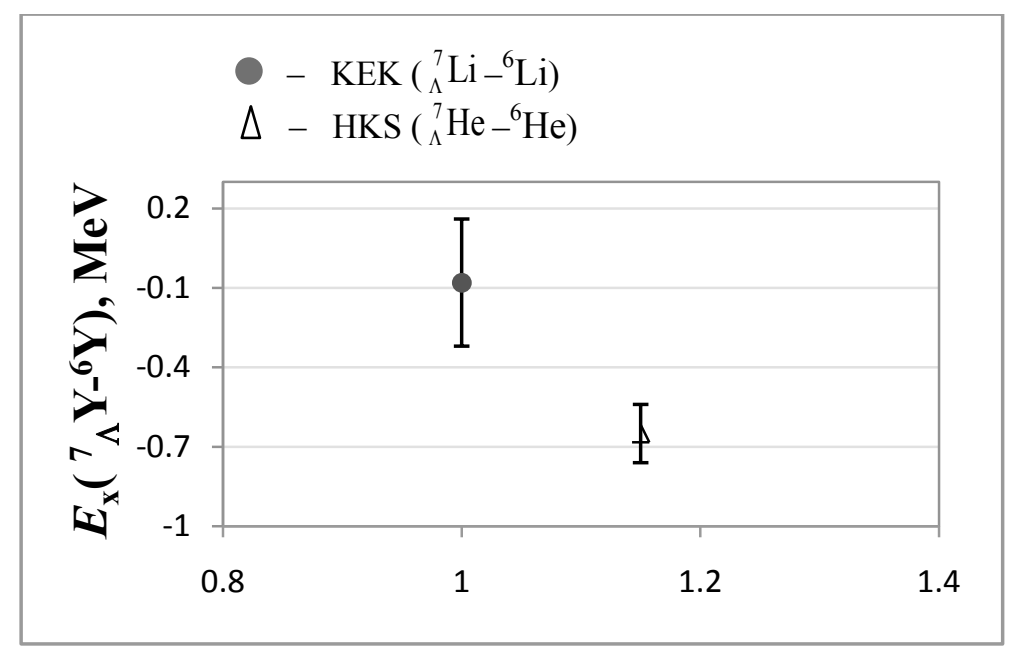

Figure 5.3.6 The change in excitation energy of the first excited and ground states of lithium and helium based on experiments E336 (KEK) and E01-011 (HKS). 


\subsection{Spectroscopy of ${ }_{\Lambda}^{28} \mathrm{Al}$ hypernuclei}

\section{Population of the states}

The hypernuclear states of ${ }_{\Lambda}^{28} \mathrm{Al}$, which are produced in the reaction, are obtained by the coupling of a $\Lambda$ with the ${ }^{27} \mathrm{Al}$ nuclear core. The ground state of ${ }^{27} \mathrm{Al}$ is characterized by a proton hole configuration in the $d_{5 / 2}$ shell, thus total spin $5 / 2$ and positive parity. The ${ }^{27} \mathrm{Al}$ ground state couples with the $\Lambda$ in $s, p$, and $d$ shells. This coupling results in three doublets $\left(2^{+}, 3^{+}\right),\left(4^{-}, 3^{-}\right)$, and $\left(5^{+}, 4^{+}\right)$that correspond to ${ }^{27} \mathrm{Al}\left(5 / 2^{+}, d\right) \otimes \Lambda\left(1 / 2^{+}, s\right),{ }^{27} \mathrm{Al}$ $\left(5 / 2^{+}, d\right) \otimes \Lambda\left(3 / 2^{-}, p\right.$ and $\left.1 / 2^{-}, p\right)$ and ${ }^{27} \mathrm{Al}\left(5 / 2^{+}, d\right) \otimes \Lambda\left(3 / 2^{+}, d\right.$ and $\left.5 / 2^{+}, d\right)$, respectively. In a theoretical calculation of the excitation energy spectrum, the DWIA formalism was used in conjunction with Saclay-Lyon-A [94] and [95], Kaon-MAID [96], AdelseckSaghai [97] and Williams-Ji-Cotanch [98] isobaric models for the elementary production process and various nuclear and hypernuclear wave functions [99]. The proton-hole widths were employed tentatively and ranged from 0 to $10 \mathrm{MeV}$. For the $\Lambda$ bound states the width was chosen to coincide with the predicted energy resolution, $\Gamma_{\Lambda}(J)=0.3 \mathrm{MeV}$ and in the unbound region was taken to be more than $1 \mathrm{MeV}$, rapidly increasing further into the quasi-free region.

The theoretical predictions are summarized in Table 5.4.1. The predicted binding energy spectrum is shown in Fig. 5.4.1. The three ground state doublets are present with relatively high strength together with lower strength peaks in between. These relatively small peaks represent the core-excited states, the detailed analysis of which will be given further in the text. The relative strengths of the states in each angular momentum 
multiplet ( $J$-multiplet) are shown in Fig. 5.4.2. The dominating factor in the separation between the individual peaks in the $J$-multiplets is the spin-orbit splitting, which is very small. That is because the particle-hole interactions with the high-spin states are generally quite small. For a $\Lambda$ hyperon in the $p$ orbital, the ${ }^{27} \mathrm{Al}\left(5 / 2^{+}, d\right) \otimes \Lambda\left(3 / 2^{-}, p\right)=4^{-}$and ${ }^{27} \mathrm{Al}$ $\left(5 / 2^{+}, d\right) \otimes \Lambda\left(1 / 2^{-}, p\right)=3^{-}$states are dominantly populated, which provides a good opportunity to observe the spin-orbit $(l s)$ splitting. When planning the experiment, this splitting had been assumed to be larger than shown in Fig. 5.4.1. A simulated ${ }_{\Lambda}^{28} \mathrm{Al}$ binding energy spectrum based on older predictions with $l_{s}$ strength of $V_{s o}=2 \mathrm{MeV}$ and FWHM energy resolution of $300 \mathrm{keV}$ is shown in Fig. 5.4.3. Results will be compared to both predictions.

Table 5.4.1 Theoretical calculations of ${ }_{\Lambda}^{28} \mathrm{Al}$ energy spectrum with cross sections [99].

\begin{tabular}{cccccc}
\hline $\begin{array}{c}{ }^{27} \mathrm{Al}, J^{p} \\
(\text { shell })\end{array}$ & $\Lambda, J^{p}$ (shell) & ${ }_{\Lambda}^{28} \mathrm{Al}, J^{p}$ & $\begin{array}{c}\text { Theor. excitation } \\
\text { energy, } E_{x}(\mathrm{MeV})\end{array}$ & $\begin{array}{c}\text { Theor. cross sections (nb/sr), } \theta_{K}=3^{\circ} \\
\text { SLA }\end{array}$ & KMAID \\
\hline \multirow{2}{*}{$5 / 2^{+}(d$, g.s. $)$} & $1 / 2^{+}(s)$ & $2^{+}$ & 0.0 & 19.8 & 14.3 \\
& $1 / 2^{+}(s)$ & $3^{+}$ & & 39.4 & 28.1 \\
\hline \multirow{2}{*}{$5 / 2^{+}(d$, g.s. $)$} & $3 / 2^{-}(p)$ & $4^{-}$ & 9.42 & 3.0 & 3.7 \\
& $1 / 2^{-}(p)$ & $3^{-}$ & 9.67 & 2.3 & 2.7 \\
\hline \multirow{2}{*}{$5 / 2^{+}(d$, g.s. $)$} & $3 / 2^{+}(d)$ & $4^{+}$ & 17.6 & 3.0 & 3.7 \\
& $5 / 2^{+}(d)$ & $5^{+}$ & 19.9 & 2.3 & 2.7 \\
\hline
\end{tabular}




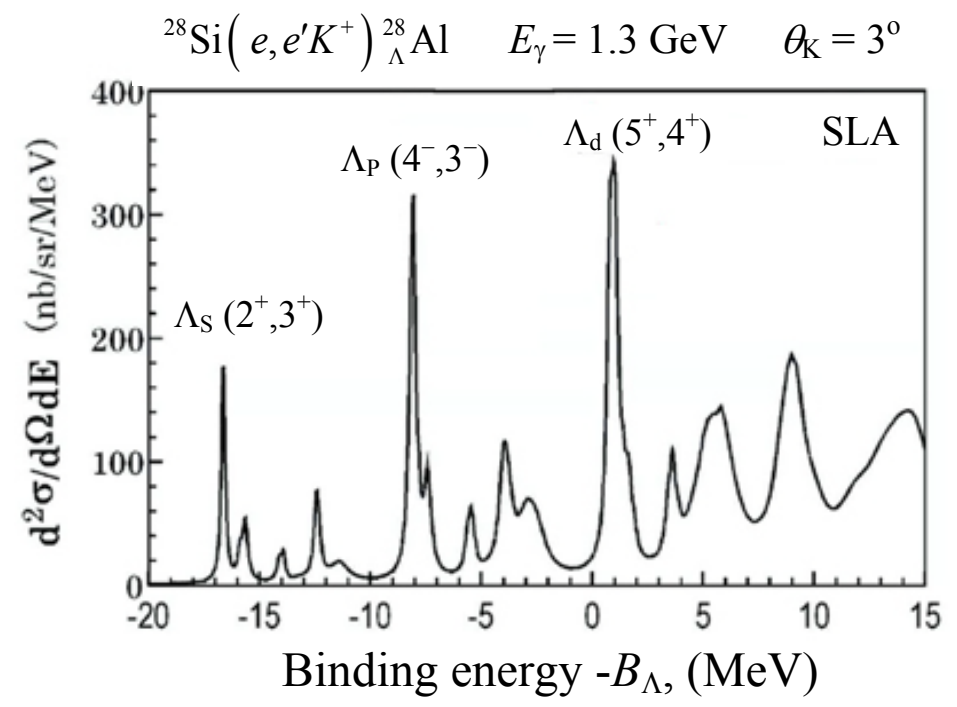

Figure 5.4.1 Binding energy $\left(-B_{\Lambda}\right)$ prediction for ${ }_{\Lambda}^{28} \mathrm{Al}$ hypernuclei [99].

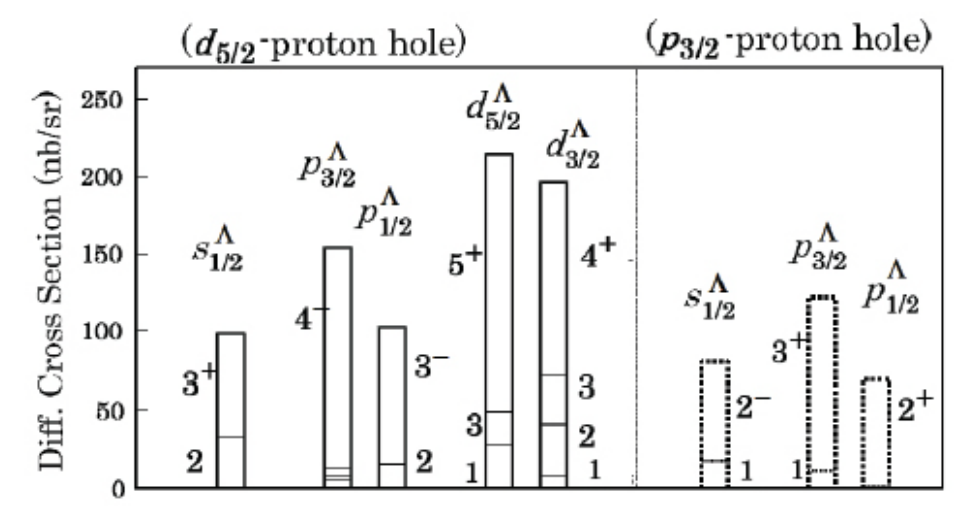

Figure 5.4.2 Divided contributions to the particle hole $J$-multiplet state for ${ }^{28} \mathrm{Si}\left(e, e^{\prime} K^{+}\right){ }_{\Lambda}^{28} \mathrm{Al}$ reaction at $E_{\gamma}=1.3 \mathrm{GeV}$ and $\theta_{K^{+}}^{L A B}=3^{\circ}$ with each pillar corresponding to differential cross section (SLA) [99]. 


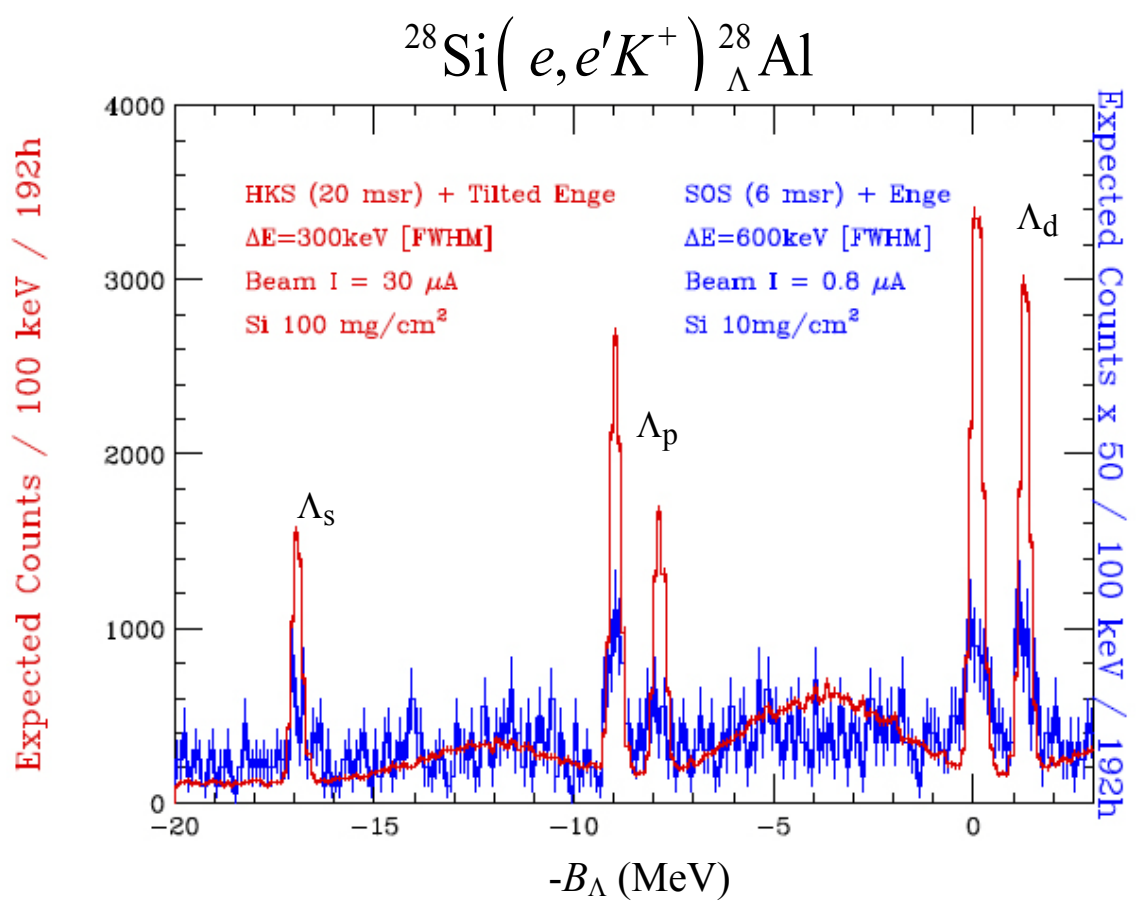

Figure 5.4.3 Simulated binding energy $\left(-B_{\Lambda}\right)$ spectrum of ${ }_{\Lambda}^{28} \mathrm{Al}$ hypernuclei to be observed in E01-011 experiment.

\section{Spectroscopy}

The binding energy spectrum for ${ }_{\Lambda}^{28} \mathrm{Al}$ hypernuclei obtained in the E01-011 experiment is presented in Fig. 5.4.4. In the spectrum we clearly observe three peaks that are positioned at approximately $-18 \mathrm{MeV},-7 \mathrm{MeV}$, and $1.5 \mathrm{MeV}$ for $\Lambda_{s}, \Lambda_{p}$, and $\Lambda_{d}$, respectively. The expected spin-orbit splitting is not seen. The width of the $\Lambda_{S 1 / 2}$ state is close to $400 \mathrm{keV}$. The $p$ and $d$ shell distributions are slightly wider $\approx 600 \mathrm{keV}$, which suggests some spinorbit splitting in these doublets. According to the theoretical predictions, we can resolve the states' doublet structure only with an energy resolution less than $300 \mathrm{keV}$ (FWHM). As it was anticipated we observe an elevated structure surrounding the area of the ground state peaks. However, the relative strength of this structure is quite high in comparison to the major doublets. That might compromise the existence of the $d$-shell doublet structure, 
which we assume to observe in the unbound region at $\approx 1.5 \mathrm{MeV}$. In addition to linear background and square-root quasi-free distribution, we apply three Gaussian functions to fit the spectrum. The results of the fit together with comparison to theoretical calculations are presented in Table 5.4.2.

Table 5.4.2 Comparison of experimental results for ${ }_{\Lambda}^{28} \mathrm{Al}$ with theoretical predictions [99].

\begin{tabular}{cccccc}
\hline $\begin{array}{c}{ }^{27} \mathrm{Al}, J^{p} \\
(\text { shell })\end{array}$ & $\begin{array}{c}\Lambda, J^{p} \\
(\text { shell })\end{array}$ & ${ }^{28} \mathrm{Al}, J^{p}$ & $\begin{array}{c}\text { Exp. binding } \\
\text { energy, }-B_{\Lambda}(\mathrm{MeV})\end{array}$ & $\begin{array}{c}\text { Exp. excitation } \\
\text { energy, } E_{\mathrm{x}}(\mathrm{MeV})\end{array}$ & $\begin{array}{c}\text { Theor. excitation } \\
\text { energy, } E_{\mathrm{x}}(\mathrm{MeV})\end{array}$ \\
\hline $5 / 2^{+}(d$, g.s. $)$ & $\begin{array}{c}1 / 2^{+}(s) \\
1 / 2^{+}(s)\end{array}$ & $2^{+}$ & $\begin{array}{c}-17.864 \pm 0.027(\mathrm{st}) \\
\pm 0.13(\mathrm{sys})\end{array}$ & 0.0 & 0.0 \\
\hline $5 / 2^{+}(d$, g.s. $)$ & $\begin{array}{c}3 / 2^{-}(p) \\
1 / 2^{-}(p)\end{array}$ & $4^{-}$ & $\begin{array}{c}-6.858 \pm 0.036(\mathrm{st}) \\
\pm 0.11(\mathrm{sys})\end{array}$ & $11.006 \pm 0.131$ & 9.42 \\
\hline $5 / 2^{+}(d$, g.s. $)$ & $3 / 2^{+}(d)$ & $4^{+}$ & $\begin{array}{c}1.336 \pm 0.054(\mathrm{st}) \\
\pm 0.10(\mathrm{sys})\end{array}$ & $19.20 \pm 0.145$ & 17.6 \\
& $5 / 2^{+}(d)$ & $5^{+}$ & & & \\
\hline
\end{tabular}

By setting the first ground state doublet with $\Lambda_{s}$ configuration to zero we obtain the excitation energies for the rest of the ground state doublets. As we can see, the experimental values exceed the predicted ones by at least $1.5 \mathrm{MeV}$.

As we mentioned earlier, several low strength peaks predicted in the ${ }_{\Lambda}^{28} \mathrm{Al}$ energy spectrum in between the prominent ground state structures correspond to core-excited state configurations. The level diagram for the nuclear host ${ }^{27} \mathrm{Al}$ is shown in Fig. 5.4.5. 


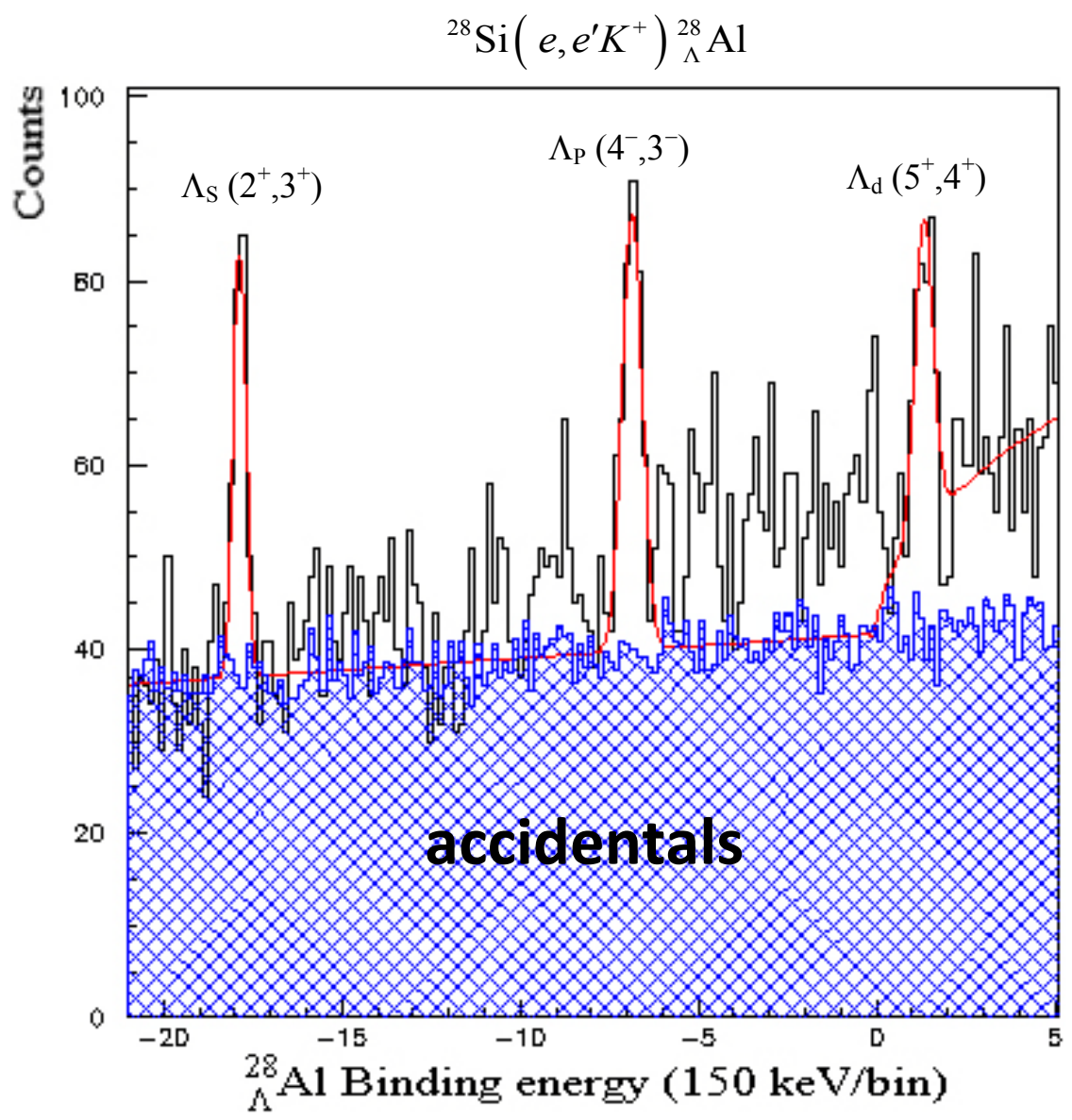

Figure 5.4.4 Experimental binding energy spectrum $\left(-B_{\Lambda}\right)$ of ${ }_{\Lambda}^{28} \mathrm{Al}$ hypernucleus.

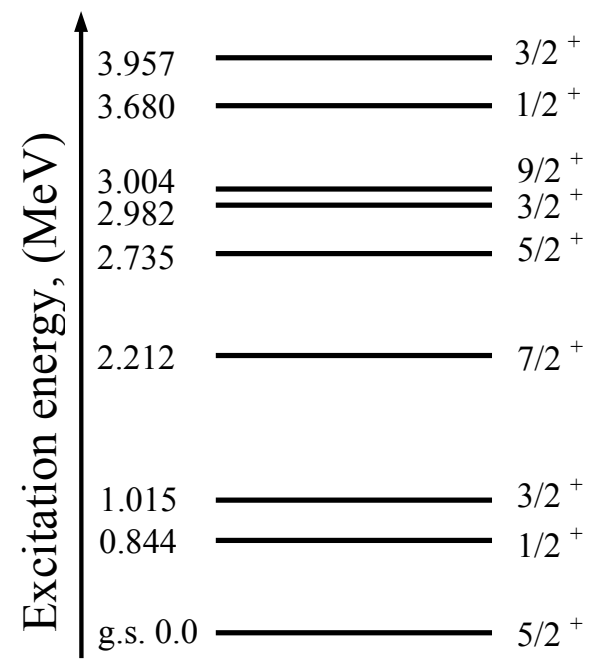

Figure 5.4.5 Level scheme for ${ }^{27} \mathrm{Al}$ nucleus. 


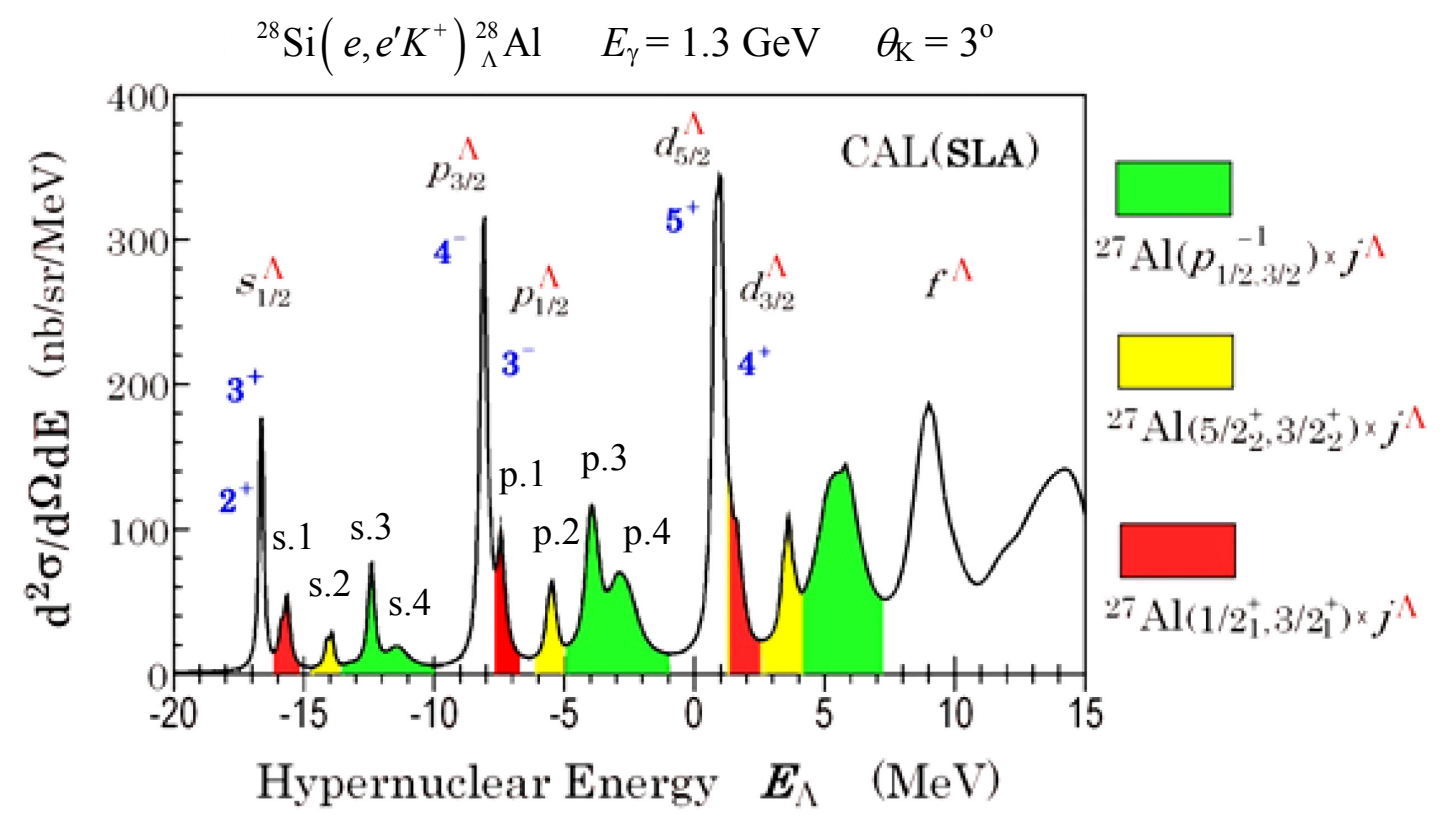

Major peak series : $\left[{ }^{2} \overline{\mathrm{Al}}\left(5 / 2_{1}^{+}\right) \times j^{\wedge}\right]_{J}$ with $j^{\wedge}=s, p, d, \ldots$

Figure 5.4.6 Predicted hypernuclear spectrum of ${ }_{\Lambda}^{28} \mathrm{Al}$ with marked core-excited states configurations [56].

By comparing the ${ }^{27} \mathrm{Al}$ level scheme with the predicted binding energy spectrum of the ${ }_{\Lambda}^{28} \mathrm{Al}$ hypernucleus one can assume the configuration of the core-excited states shown in Fig. 5.4.6. The $\Lambda$ particle in the $s, p$, and $d$ shells couples first to $\left(1 / 2^{+}, 3 / 2^{+}\right)$ doublet and further to $\left(5 / 2^{+}, 3 / 2^{+}\right)$and $\left(1 / 2^{+}, 3 / 2^{+}\right)$excited states.

In the spectrum presented in Fig. 5.4.4 we definitely observe the high strength structure between $p$ and $d \Lambda$ states configurations. Similar lower strength distributions are present between $s$ and $p \Lambda$ states. To analyze the core-excited structure in the unbound region that already includes $\Lambda_{d}$ ground state peak we have to understand very well the shape of the quasi-free distribution. That requires a stand-alone simulation and extended study. At the present time we will only concentrate on an analysis of the core-excited 
states in the bound region, between the major $d, p$, and $s$ peaks. The fit presented in Fig. 5.4.7 includes four Gaussians between $\Lambda_{s}$ and $\Lambda_{p}$ and another four between $\Lambda_{p}$ and $\Lambda_{d}$. The states are marked "s.1", "s.2", “s.3", "s.4" and "p.1", “p.2", "p.3", "p.4” on both figures, 5.4.6 and 5.4.7. The results of the fit are summarized in Table 5.4.3.

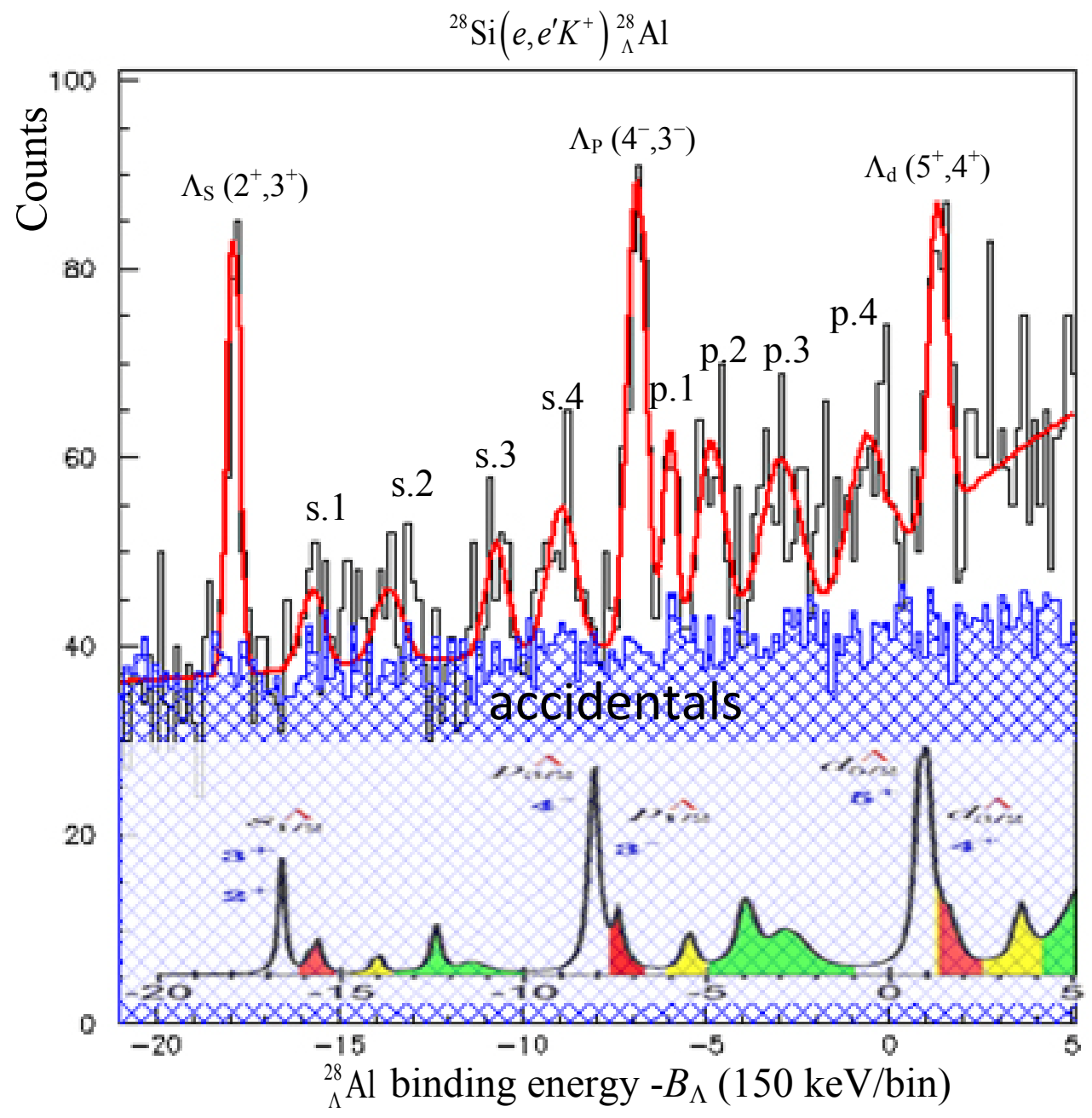

Figure 5.4.7 Binding energy spectrum $\left(-B_{\Lambda}\right)$ of ${ }_{\Lambda}^{28} \mathrm{Al}$ hypernucleus with core-excited states. 
Table 5.4.3 Fitting results for ${ }_{\Lambda}^{28} \mathrm{Al}$ spectrum.

\begin{tabular}{lccccc}
\hline Structure & shells & Exp., $-B_{\Lambda}(\mathrm{MeV})$ & Exp., $E_{\mathrm{x}}(\mathrm{MeV})$ & Peak strength FWHM, MeV \\
\hline${ }^{27} \mathrm{Al}\left(5 / 2^{+} \mathrm{g} . \mathrm{s}.\right) \otimes \Lambda\left(1 / 2^{+} \mathrm{s}\right)$ & $\left(2^{+}, 3^{+}\right)$ & $-17.864 \pm 0.027$ & $0 \pm 0.027$ & $33.62 \pm 8.75$ & $0.392 \pm 0.073$ \\
\hline${ }^{27} \mathrm{Al}\left(1 / 2^{+}{ }_{0.8}, 3 / 2^{+}{ }_{1.0}\right) \otimes \Lambda\left(1 / 2^{+} \mathrm{s}\right)$ & $\mathrm{s} .1$ & $-15.728 \pm 0.258$ & $2.136 \pm 0.259$ & $11.05 \pm 5.24$ & 0.75 (at limit) \\
\hline${ }^{27} \mathrm{Al}\left(5 / 2^{+}{ }_{2.7}, 3 / 2^{+}{ }_{2.9}\right) \otimes \Lambda\left(1 / 2^{+} \mathrm{s}\right)$ & $\mathrm{s} .2$ & $-13.651 \pm 0.325$ & $4.213 \pm 0.325$ & $40.77 \pm 31.06$ & 0.92 (at limit) \\
\hline${ }^{27} \mathrm{Al}\left(1 / 2^{+}{ }_{3.6}, 3 / 2^{+}{ }_{3.9}\right) \otimes \Lambda\left(1 / 2^{+} \mathrm{s}\right)$ & $\mathrm{s} .3$ & $-10.709 \pm 0.125$ & $7.155 \pm 0.128$ & $15.37 \pm 12.24$ & $0.712 \pm 0.523$ \\
& $\mathrm{~s} .4$ & $-8.924 \pm 0.134$ & $8.940 \pm 0.137$ & $25.13 \pm 5.43$ & 0.92 (at limit) \\
${ }^{27} \mathrm{Al}\left(5 / 2^{+} \mathrm{g} . \mathrm{s}.\right) \otimes \Lambda\left(3 / 2^{-} \mathrm{p}, 1 / 2^{-} \mathrm{p}\right)$ & $\left(4^{-}, 3^{-}\right)$ & $-6.865 \pm 0.035$ & $10.999 \pm 0.044$ & $52.61 \pm 10.23$ & $0.598 \pm 0.088$ \\
${ }^{27} \mathrm{Al}\left(1 / 2^{+}{ }_{0.8}, 3 / 2^{+}{ }_{1.0}\right) \otimes \Lambda\left(3 / 2^{+} \mathrm{p}, 1 / 2^{+} \mathrm{p}\right)$ & $\mathrm{p} .1$ & $-5.964 \pm 0.058$ & $11.900 \pm 0.064$ & $15.86 \pm 6.26$ & $0.396 \pm 0.107$ \\
${ }^{27} \mathrm{Al}\left(5 / 2^{+}{ }_{2.7}, 3 / 2^{+}{ }_{2.9}\right) \otimes \Lambda\left(3 / 2^{+} \mathrm{p}, 1 / 2^{+} \mathrm{p}\right)$ & $\mathrm{p} .2$ & $-4.838 \pm 0.088$ & $13.026 \pm 0.092$ & $32.33 \pm 10.23$ & $0.864 \pm 0.198$ \\
${ }^{27} \mathrm{Al}\left(1 / 2^{+}{ }_{3.6}, 3 / 2^{+}{ }_{3.9}\right) \otimes \Lambda\left(3 / 2^{+} \mathrm{p}, 1 / 2^{+} \mathrm{p}\right)$ & $\mathrm{p} .3$ & $-2.935 \pm 0.156$ & $14.929 \pm 0.158$ & $44.51 \pm 6.84$ & 1.33 (at limit) \\
& $\mathrm{p} .4$ & $-0.571 \pm 0.131$ & $17.293 \pm 0.134$ & $48.77 \pm 6.98$ & 1.33 (at limit) \\
${ }^{27} \mathrm{Al}\left(5 / 2^{+}\right.$g.s. $) \otimes \Lambda\left(5 / 2^{+} \mathrm{p}, 3 / 2^{+} \mathrm{p}\right)$ & $\left(5^{+}, 4^{+}\right)$ & $1.342 \pm 0.053$ & $19.209 \pm 0.059$ & $35.10 \pm 8.57$ & $0.584 \pm 0.088$ \\
\hline
\end{tabular}

The parameters of the Gaussian functions for all three ground-state peaks have been allowed to vary freely, while the parameters for the $s$ and $p$ core-excited structures have been partially bound. The initial mean values for core-excited Gaussians were chosen to coincide with the theoretical prediction, and during the fit a $\pm 1.5 \mathrm{MeV}$ variation was permitted. The peak widths have also been limited to approximately $2 \sigma$ of that of the ground state.

The fit results are compared at the bottom of Fig. 5.4.7 with the theoretically predicted spectrum (Fig. 5.4.6), proportionally scaled to the horizontal axis. We can see significant discrepancies in the positions of the major peaks, which are in the order of 1 to $2 \mathrm{MeV}$. The core-excited structure (s.\#) between $s$ and $p \Lambda$ configurations is almost random. Two peaks, s.3 and s.4, have significant strength with relatively high uncertainties (Table 5.4.3). The theoretical calculations, however, have no predictions in the vicinity of these peaks. According to the "Peak strength" column in Table 5.4.3, the most reliable peak in s.\# series is s.4. It's reasonably small uncertainty combined with its 
significant strength suggests that this peak represents a true state. Because of the broad width of the peak it makes sense to assume the presence of the doublet configuration.

According to calculations, one can observe the core-excited states within $E_{x}=3$ $\mathrm{MeV}$ from the $\Lambda_{s}$ ground state. As we can see, the relatively low statistics structure that suggests the presence of peaks s. 1 and s. 2 is in poor agreement with such a statement. Beyond the $\Lambda_{p}$ ground state, the core-excited pattern, which tentatively has been selected to contain four peaks, does not agree with the theory as well. The drastic disagreement with the predictions can be explained by the calculation not accounting for the high-lying excited orbits, which are present in the $A=27$ nuclear core in a large amount. Nine states shown in the Fig. 5.4.5 fill the region of excitation from the ground state up to $4 \mathrm{MeV}$. Beyond this energy, up to $\approx 11 \mathrm{MeV}$, there are another 231 states, which in turn individually can couple to $\Lambda\left(1 / 2^{+}\right)$state. Provided that the $\Lambda$ coupling with the nine lowlying states in $s$ configuration already is quite complicated, it is clear what one can expect when the higher lying states are included into calculations. The mixing of all $231+9=240$ states in the energy range from 0 to $11 \mathrm{MeV}$ to $\Lambda\left(3 / 2^{+}, 1 / 2^{+}\right) p$ state doublet can result in at least twice as many final states than in the case of the $\Lambda\left(1 / 2^{+}\right) s$ configuration. We are not aware of any theoretical studies taking this into account.

As we stated above, the visual comparison of experimental results with theoretical predictions shows disagreement in the ground state peaks' locations. One of the possible explanations that can partially reinstate the credibility of the theory is another approach to the peak assignments. We can assume that the s. 4 is not a core-excited state, but the $4^{-} \Lambda_{p}$ configuration, which in turn suggests the next tall peak is the $3^{-} \Lambda_{p}$ state. From Table 
5.4.3, the distance between peaks s. 4 and $\Lambda_{\mathrm{p}}$ is about $1.8 \mathrm{MeV}$. Although it might quite conveniently explain the $2 \mathrm{MeV}$ predicted splitting in the $\Lambda_{p}$ state doublet (Figures 5.4.2 and 5.4.3), the ratio of the peak strengths in this experiment does not match the calculations. Assuming the new statement of peak identifications is correct, the experimental strength ratio $\Lambda_{p}\left(4^{-}\right.$,g.s. $) / \Lambda_{p}\left(3^{-}\right.$,g.s. $)<1$ contradicts the expected ratio, which is estimated to be more than 1 . Such controversy does not allow us to provide a firm statement on the validity of our interpretation of the states. Therefore we will leave it open for further theoretical analysis.

\section{Comparison to the mirror hypernuclei}

A similar hypernuclear structure should exist in ${ }_{\Lambda}^{28} \mathrm{Si}$, the mirror hypernucleus to ${ }_{\Lambda}^{28} \mathrm{Al}$. The production of the ${ }_{\Lambda}^{28} \mathrm{Si}$ hypernucleus was performed for the first time at the Alternating Gradient Synchrotron (AGS) at BNL in the 80's [100]. It utilized the $\left(\pi^{+}, K^{+}\right)$ meso-production reaction. The spectrum, with $2 \mathrm{MeV}$ (FWHM) energy resolution, is shown in Fig. 5.4.8. Clearly visible are the three prominent peaks with configurations $0 d_{5 / 2}^{-1} \otimes s_{1 / 2 \Lambda}\left(2_{g . s .}^{+}\right), \quad 0 d_{5 / 2}^{-1} \otimes p_{3 / 2 \Lambda, 1 / 2 \Lambda}\left(3^{-}\right)$, and $0 d_{5 / 2}^{-1} \otimes d_{\Lambda}\left(4^{+}\right.$resonance). The resolution of the spectrum is insufficient to see any fine state structure between the peaks. The results of the fits are presented in Table 5.4.4. Figure 5.4.9 displays the ${ }_{\Lambda}^{28} \mathrm{Si}$ spectrum obtained by $\left(\pi^{+}, K^{+}\right)$in the E369 experiment at KEK. Peaks \#1, \#3, and \#5 are distributed along the missing mass scale in a very similar way as in the BNL experiment. The positions of the peaks are also compatible with the ${ }_{\Lambda}^{28} \mathrm{Al}$ spectrum. In the same way as for ${ }_{\Lambda}^{28} \mathrm{Al}$, the 
${ }_{\Lambda}^{28} \mathrm{Si}$ is composed of the particle-hole ${ }^{27} \mathrm{Si}$ configuration coupling with the $\Lambda$. The only difference is that now we have a neutron-hole $J$-multiplet instead of proton-hole configuration. The fit results for the ${ }_{\Lambda}^{28} \mathrm{Si}$ spectrum are shown in Table 5.4.5 [8]. The suggestion of the core-excited configurations is also present in peaks \#2 and \#4. The resolution of these peaks, however, is on the order of few MeV. Any fine core-excited states configuration that might be in the spectrum is completely washed out by the low resolution.

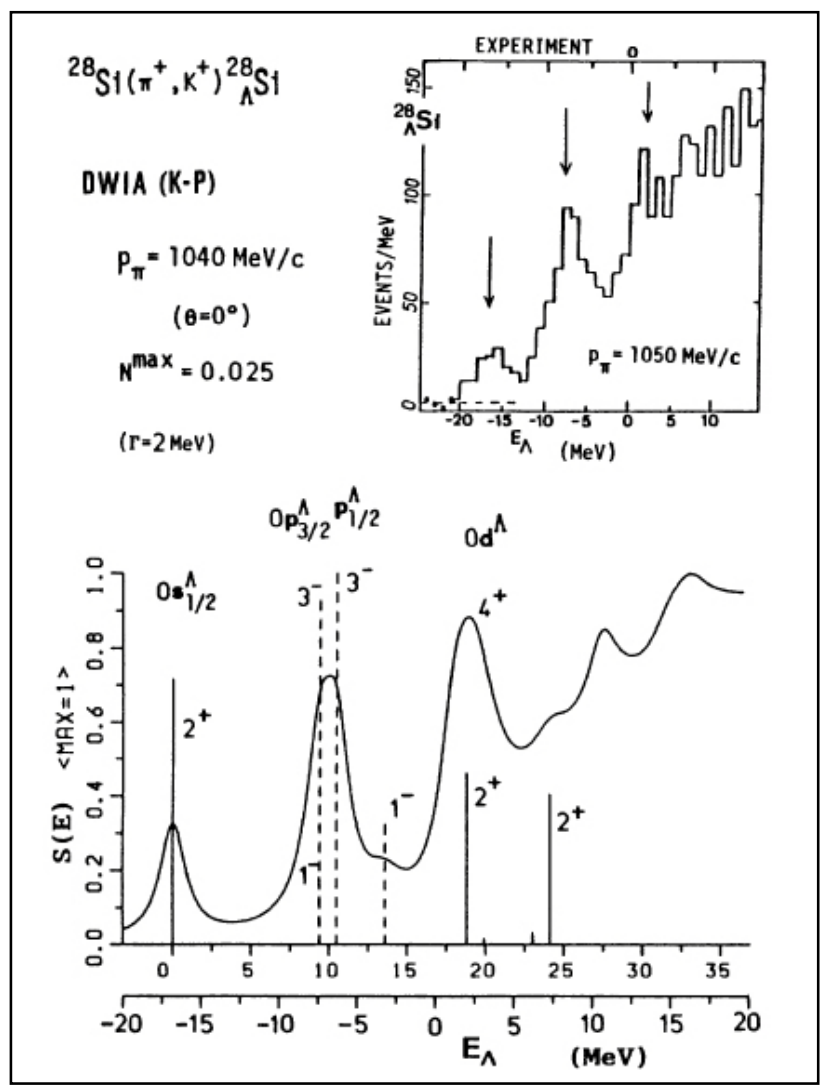

Figure 5.4.8 Experimental (top) and theoretical (bottom) excitation energy spectra of ${ }_{\Lambda}^{28} \mathrm{Si}$ measured with the AGS at BNL by $\left(\pi^{+}, K^{+}\right)$reaction [100]. 
Table 5.4.4 Fitting results for ${ }_{\Lambda}^{28} \mathrm{Si}$ spectrum obtained in AGS at BNL [100].

\begin{tabular}{cccc}
\hline Peaks & Calculated $E_{x}, \mathrm{MeV}$ & Experimental $E_{x}, \mathrm{MeV}$ & Width FWHM, (MeV) \\
\hline $0 d_{5 / 2}^{-1} \otimes s_{1 / 2 \Lambda}\left(2_{\text {g.s. }}^{+}\right)$ & -16.8 & -16 & $2 \mathrm{MeV}$ \\
\hline $0 d_{5 / 2}^{-1} \otimes p_{3 / 2 \Lambda, 1 / 2 \Lambda}\left(3^{-}\right)$ & $-7.4 ;-6.3$ & -7 & \\
\hline $0 d_{5 / 2}^{-1} \otimes d_{\Lambda}\left(4^{+}\right.$resonance $)$ & 2 & 2 & \\
\hline
\end{tabular}

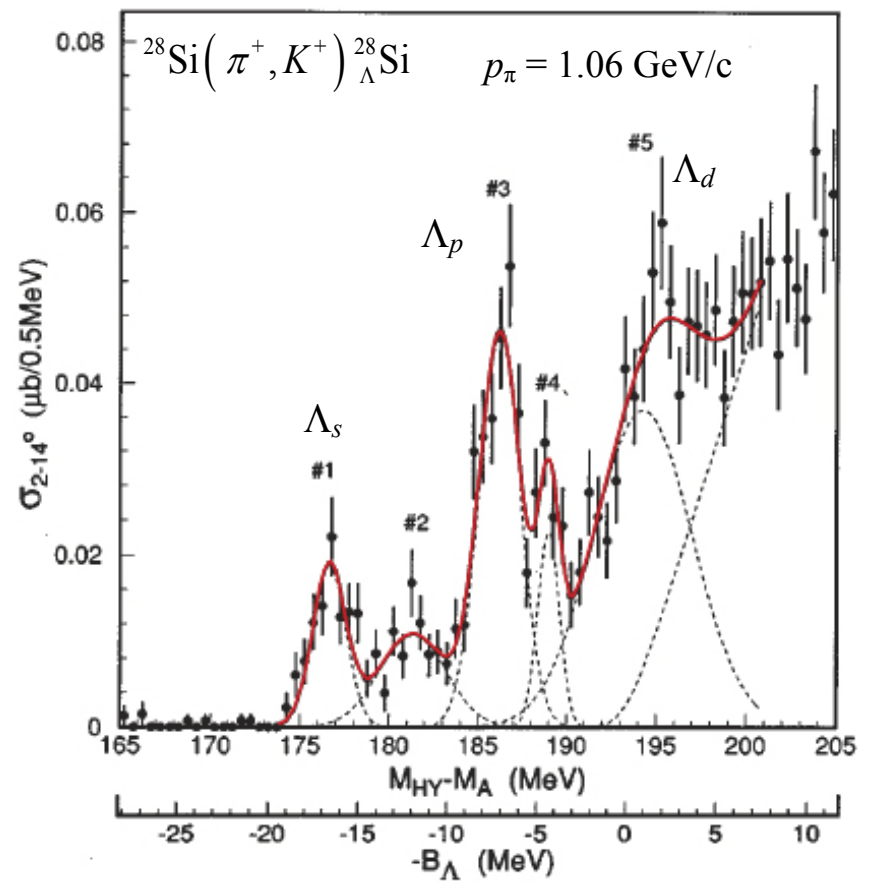

Figure 5.4.9 Excitation energy spectrum of ${ }_{\Lambda}^{28} \mathrm{Si}$ measured with the SKS spectrometer at $\operatorname{KEK}(\mathrm{E} 369)$ by $\left(\pi^{+}, K^{+}\right)$reaction [8].

Table 5.4.5 Fitting results for ${ }_{\Lambda}^{28} \mathrm{Si}$ spectrum obtained in E369 at KEK [8].

\begin{tabular}{ccccc}
\hline Peaks & $M_{\mathrm{HY}}-M_{\mathrm{A}},(\mathrm{MeV})$ & Uncertainties, $(\mathrm{MeV})$ & $B_{\Lambda},(\mathrm{MeV})$ & Width FWHM, $(\mathrm{MeV})$ \\
\hline$\# 1$ & 176.6 & \pm 0.2 & 16.6 & 2.2 (fixed) \\
\hline$\# 2$ & 181.3 & \pm 0.4 & 11.9 & $4.4 \pm 1.0$ \\
\hline$\# 3$ & 186.2 & \pm 0.2 & 7.0 & $2.7 \pm 0.3$ \\
\hline$\# 4$ & 189.0 & \pm 0.2 & 4.3 & $1.4 \pm 0.4$ \\
\hline$\# 5$ & 194.3 & \pm 0.8 & -1.0 & $6.5 \pm 1.1$ \\
\hline
\end{tabular}


To summarize, in our measurements of ${ }_{\Lambda}^{28} \mathrm{Al}$ we observe the ground state to be deeper bound by roughly $1 \mathrm{MeV}$ than predicted by theory and also observed for the mirror hypernucleus ${ }_{\Lambda}^{28} \mathrm{Si}$. The separation between $\Lambda s$ and $p$ shell orbits is about $1.4 \mathrm{MeV}$ bigger in ${ }_{\Lambda}^{28} \mathrm{Al}$ than in ${ }_{\Lambda}^{28} \mathrm{Si}$. Remarkably the $p$ and $d$ shell separation differs between both spectra by $0.2 \mathrm{MeV}$, which is within experimental uncertainties. The ambiguity of our ${ }_{\Lambda}^{28} \mathrm{Al}$ data in the core-excited region together with the poor resolution of the previously measured ${ }_{\Lambda}^{28} \mathrm{Si}$ does not allow for more detailed comparisons.

\subsection{Discussion}

This chapter presented the analysis of high resolution spectra of the ${ }_{\Lambda}^{12} \mathrm{~B}$, exotic neutronrich ${ }_{\Lambda}^{7} \mathrm{He}$, and medium-mass number ${ }_{\Lambda}^{28} \mathrm{Al}$ hypernuclei. Each of these hypernuclei presents interesting physics results. The uniqueness of the ${ }_{\Lambda}^{12} \mathrm{~B}$ hypernuclear spectrum is the population of the spin-flip unnatural parity states with high strength, primarily made possible because of the intense electron beam at CEBAF. Such states have never been observed with meso-production reactions. The first time the binding energy of the ${ }_{\Lambda}^{12} \mathrm{~B}$ ground state, $B_{\Lambda}=11.37 \pm 0.06 \mathrm{MeV}$, was measured in an emulsion experiment [90].

The relatively recent (late 90's) experiments at KEK delivered the light- to medium-mass hypernuclear spectra with the best resolution of $1.5 \mathrm{MeV}$. The core-excited structure was observed, for example in the spectrum for ${ }_{\Lambda}^{12} \mathrm{C}$ (Fig. 5.2.5). However, because of poor resolution it was difficult to precisely determine their positions. The high 
resolution spectroscopy of the present experiment delivered the best available FWHM energy resolution, not only for high statistic ground states, but also for rare core-excited states. By achieving $\approx 0.4 \mathrm{MeV}$ energy resolution in the current experiment we set the record in hypernuclear reaction spectroscopy. Although it is not enough to resolve the spin-orbit splitting in doublet state configurations, it provides the best measurements for binding energies. The results from our $\left(e, e^{\prime} K^{+}\right)$experiments combined with the recent $\gamma$ ray spectroscopy experiments at KEK, where the relative positions of the states can be measured with high precision, serve as a sanity probe for the theoretical models. In this dissertation I did a detailed analysis of the core-excited states of the ${ }_{\Lambda}^{12} \mathrm{~B}$ hypernucleus and presented a comparison with the mirror ${ }_{\Lambda}^{12} \mathrm{C}$ hypernucleus. As a result of the applied fit in our ${ }_{\Lambda}^{12} \mathrm{~B}$ spectrum, the excitation and binding energies of the first and second coreexcited states have been found. The numbers were consistent with the mirror hypernuclear system. The binding properties of a $\Lambda$ embedded into the nuclear core have been observed.

The hypernuclear spectrum of ${ }_{\Lambda}^{7} \mathrm{He}$ has been expected for a long time in reaction spectroscopy of light neutron-rich hypernuclei. Inconclusive results in pion decay emulsion experiments [90] motivated experimentalists to obtain this spectrum to complete the $A=7$ isospin $I=1$ triplet. By studying the iso-triplet one can learn things about three body forces in $\Lambda \mathrm{N}-\Sigma \mathrm{N}$ coupling and investigate charge symmetry breaking effect, which might be highly pronounced because of the halo configuration of the $A=7$ hypernuclear system. The statistically significant ${ }_{\Lambda}^{7} \mathrm{He}$ spectrum obtained in this 
experiment allowed reliable measurement of the binding energy for the first time in the history of hypernuclear physics. The spectrum fit resulted in $B_{\Lambda}=5.73 \pm 0.12 \mathrm{MeV}$ with an excellent energy resolution of $0.47 \mathrm{MeV}$ (FWHM). With such resolution it is possible to observe the core-excited state configurations $\left(3 / 2^{+}, 5 / 2^{+}\right)$that were predicted to be bound by $1.7 \mathrm{MeV}$ less than the ground state $\left(1 / 2^{+}\right)$. As a result of our analysis we were able to extract the energy of the core-excited doublet, however, the correctness of it might be fairly questioned due to a low statistical significance. The present energy resolution in the spectrum does not allow resolving the spin-orbit splitting, which might manifest itself in a core-excited doublet, due to exactly the same reason as for ${ }_{\Lambda}^{12} \mathrm{~B}$ hypernuclei. $\mathrm{A}$ resolution of at least $100 \mathrm{keV}$ is required for identification of individual members of closely positioned $J$-multiplets. The comparison of the results obtained in our experiment on ${ }_{\Lambda}^{7} \mathrm{He}$ and measured at KEK for the ${ }_{\Lambda}^{7} \mathrm{Li}$ spectra revealed interesting properties of $\Lambda \mathrm{N}$ interactions.

The ${ }_{\Lambda}^{28} \mathrm{Al}$ represents the medium-mass-number $A=28$ hypernucleus, where the structure of the states likely become complicated in comparison to light hypernuclear systems. Until now the spectrum for ${ }_{\Lambda}^{28} \mathrm{Al}$ has not been available. The meson beams at KEK and BNL resulted in obtaining good quality spectra for the mirror hypernuclei, ${ }_{\Lambda}^{28} \mathrm{Si}$.

The spectrum of ${ }_{\Lambda}^{28} \mathrm{Si}$ was expected to have very similar distribution of the major shell structure due to a particle-hole configuration and mirror proton to neutron ratio. Although the three major peaks have been clearly identified, the previous $2 \mathrm{MeV}$ resolution did not allow for resolving any fine structure of the possible core-excited states. The ${ }_{\Lambda}^{28} \mathrm{Al}$ 
spectrum obtained in the present study clearly identified the three major peaks with excellent energy resolution of $0.4 \mathrm{MeV}$ in the ground state and $0.6 \mathrm{MeV}$ for $\Lambda_{p}$ and $\Lambda_{d}$ doublets. The extra $0.2 \mathrm{MeV}$ in comparison to ground state $\Lambda_{s}$ indirectly confirms the presence of closely positioned peaks for $\Lambda_{p}$ and $\Lambda_{d}$ hypernuclear states. Of course such a statement is valid only if we assumed the same energy resolution $\approx 0.4 \mathrm{MeV}$ for each of the peaks in the doublets. The visible strength between $\Lambda_{s}$ and $\Lambda_{p}$ together with the strongly pronounced structure between $\Lambda_{p}$ and $\Lambda_{d}$ were interpreted as core-excited states. A tentative fit of the core-excited configurations for both regions showed the possible presence of eight peaks. Although this number is quite uncertain due to low statistical significance of those peaks, it is worth noting that there are more than $240 \cdot 2=480$ possible core-excited couplings along the bound region. The excitation energy predictions for core-excited configurations come from proton pick-up reactions, for example ${ }^{28} \mathrm{Si}\left(d,{ }^{3} \mathrm{He}\right){ }^{27} \mathrm{Al}$, where the spectroscopic factors are extracted from ${ }^{27} \mathrm{Al}$ spectrum and accounted for in ${ }_{\Lambda}^{28} \mathrm{Al}$ hypernuclei. Similarly the proton pick-up reactions ${ }^{12} \mathrm{C}(p, 2 p){ }^{11} \mathrm{~B}$, ${ }^{12} \mathrm{C}\left(d,{ }^{3} \mathrm{He}\right){ }^{11} \mathrm{~B}$, and ${ }^{12} \mathrm{C}\left(e, e^{\prime} p\right){ }^{11} \mathrm{~B}$ have been used for predicting the core-excited states of ${ }_{\Lambda}^{12} \mathrm{~B}$ hypernuclei.

At this time, the spectrum of ${ }_{\Lambda}^{28} \mathrm{Al}$ hypernuclei brings to hypernuclear spectroscopy the first high resolution observation of $\Lambda_{d}$ shell structure. Previously achieved spectra had several MeV FWHM resolution that resulted in great difficulty of identifying the true location of the state. Despite the relatively low statistical significance of the $d$-shell $\Lambda$ structure the precision of the excitation energy measurement is high. The 
comparison with the theoretical predictions in Chapter 5.4 revealed the strong inconsistency and allowed us to provide several interpretations. 


\section{CONCLUSIONS}

The present study provided a rigorous data analysis of the E01-011 (HKS) experiment conducted at the Thomas Jefferson National Accelerator Facility, Newport News, VA in Fall 2005. The obtained energy resolution (FWHM) of all three spectra represents the best ever achieved result in hypernuclear reaction spectroscopy. The high resolution of the hypernuclear spectra was the primary scope of our experiment. As shown it was successfully realized. Secondly, the spectrum of neutron rich ${ }_{\Lambda}^{7} \mathrm{He}$ hypernuclei has been measured for the first time. The halo configuration of this hypernuclear system gives access to interesting few-body physics and completes the missing link in the experimental data for the $A=7, I=1$ iso-spin triplet. Further, the hypernuclear spectroscopy of medium mass number can provide valuable information on $d$-shell structure. In this experiment,

the $\Lambda_{d}$ state has been clearly identified in the ${ }_{\Lambda}^{28} \mathrm{Al}$ spectrum. The $d$-shell structure was measured with sub-MeV resolution for the first time.

For all three targets we were able to extract the core-excited configurations. Although the likelihood PID method suffered some inefficiency in the missing mass spectra, we were able to obtain an excellent identification of the particles throughout the process of data analysis. Our tabulated experimentally extracted values for core-excited states will serve as a primary source for theoreticians to test the reliability of their models.

In conclusion, the second generation of the $\left(e, e^{\prime} K^{+}\right)$hypernuclear spectroscopy experiments proved itself to be a powerful tool for studying the properties of light and 
medium hypernuclear systems. It is worth mentioning that recently the new third generation experiment, E05-115, has been performed at Jefferson Lab. The ENGE spectrometer has been exchanged with a new high resolution electron spectrometer (HES) and new detectors were added to the HKS side to increase the PID power. The experiment increased the energy of the incident beam, which required the usage of a new splitter magnet to compensate for kinematics changes. The goal of the experiment was to further increase production yields and to measure heavier hypernuclear systems. The analysis of E05-115 experiment is currently underway. 


\section{REFERENCES}

1. D.E. Lanskoi, Physics of the hypernuclei, UNC DO, Moscow, (2002).

2. B. Povh, K. Rith, Particles and Nuclei, An Introduction to the Physical Concepts, (2nd ed.), Springer, Berlin, Gernamy, (1999).

3. C. Amsler et al. (Particle Data Group), PL B667, 1, (2008).

4. O. Hashimoto and H. Tamura, Prog. Part. Nucl. Phys. 57, 564, (2006).

5. D. H. Davis and J. Pniewski, Contemp. Phys. 27, 91 (1986); D. H. Davis, Nucl. Phys. A 754, 3c, (2005).

6. H. Bando, T. Motoba, and J. Zofka, Int. J. Mod. Phys. A 5, 4021, (1990).

7. R. H. Chrien, Table of observed $\Lambda$ hypernuclei (National Data Center, 2000).

8. T. Hasegawa, et al., Phys.Rev.C53, 1210, (1996).

9. D. J. Millener, C. B. Dover, A. Gal, Phys. Rev. C 38, 2700, (1988).

10. C. B. Dover, Proceedings of the International Symposium on Medium Energy Physics, Beijing, June, (1987).

11. M. Danysz and J. Pniewski, Philos. Mag. 44, 348, (1953).

12. T. Miyoshi et al., Phys. Rev. Lett. 90, 232502-1, (2003).

13. Nozawa S. and Lee T.-S. H., Nucl. Phys. A 513, 511, (1990).

14. Amalti E., Fubini S., and Furlan G., Pion-electroproduction. Springer Tracts in Modern Physics, Vol. 83, Springer, Berlin, (1979).

15. J. Adam et al., Czech. J. Phys. 42,1167, (1992).

16. T.W. Donnely, Electron and Photon Interactions at Intermediate Energies, Proceedings of the 1984 Workshop Held at Bad Honnef, Germany October 29-31, (1984). Lecture Notes in Physics, vol. 234, p.309 (1984).

17. R. A. Adelseck, C. Bennhold, and L. E. Wright, Phys. Rev. C32, 1681, (1985).

18. E.H. Auerbach et al., Hypernuclear Spectroscopy in the p-shell, Ann. of Phys. 148 381, (1983). 
19. S. Cohen and D Kurath, Nucl. Phys. 73, 1, (1965); Nucl. Phys. A101, 1, (1967).

20. M.M. Nagels et al., Phys. Rev. D 15, 2547, (1977); Phys. Rev. D 20, 1633, (1979).

21. P.M. Maessen et al., Phys. Rev. C 40, 2226, (1989).

22. T.A. Rijken et al., Phys. Rec. C 59, 21, (1999).

23. B. Holzenkamp et al., Nucl. Phys. A 500, 458, (1989).

24. K. Holinde, Nucl. Phys. A 547, 245c, (1992).

25. Y. Yamamoto et al., Prog. Theor. Phys. Suppl. 118, 361, (1994).

26. D.J. Millener et al. Phys. Rev. C 31, 499, (1985).

27. R.H. Dalitz and A. Gal. Ann. Phys. 116, 167, (1978).

28. B. Povh, Annual Review of Nuclear and Particle Science, V. 28, p. 1-32, (1978).

29. B. A. Khrylin, Hypernuclei and $\Lambda$ N Interaction, SOV PHYS USPEKHI, 14 (5), 616, (1972).

30. R.C. Herndon, Y.C. Tang, Phys. Rev. 165,1091 (1967); Phys. Rev. 165,1093 (1968).

31. A. Gal, Adv. Nucl. Phys. 8, 1, (1975).

32. A.R. Bodmer, S. Ali, Nucl. Phys. 56, 657, (1964).

33. R.C. Herndon, Y.C. Tang, Phys. Rev. 149, 753, (1966).

34. A.R. Bodmer, J.M. Murphy, Nucl. Phys. 73, 664 (1965); Nucl. Phys. 64, 593 (1965).

35. D.J. Millener, Hypernuclear Gamma-Ray Spectroscopy and the Structure of $p$-shell Nuclei and Hypernuclei, Lect. Notes Phys. 724, 31, (2007).

36. P. H. Pile et al., Phys. Rev. Lett. 66, 2585, (1991).

37. T. Motoba, Nucl. Phys. A 639, 135c, (1998).

38. H. Hotchi et al., Phys. Rev. C64, 044302, (2001).

39. O. Hashimoto, J. Reinhold, L. Tang, S. Nakamura, E01-011 (HKS) experiment proposal, JLab PAC19, (2001).

40. C.W. Leeman, D.R. Douglas, G.A. Kraft, Annu. Rev. Nucl. Part. Sci. 51, 413-50, (2001). 
41. C. Yan, P. Adderley, D. Barker et al., Superharp - A wire scanner with absolute position readout for beam energy measurement at CEBAF, Nucl. Inst. Meth. A365, 261, (1995).

42. C. Yan, R. Carlini, D. Neuffer, Beam energy measurement using the Hall C beamline, Prepared for 1993 IEEE Particle Accelerator Conference (PAC 93), Washington, DC, 17-20 May (1993).

43. L. H. Harwood, et al., CEBAF-PR-89-006, (1989).

44. P.Chevtsov et al., Proceedings of the 2003 Particle Accelerator Conference, Portland, OR, (2003).

45. P.Chevtsov et al., Proceedings of ICALEPCS2003, Gyeongju, Korea.

46. P. Chevtsov et al., Nucl. Instr. Meth. A557, 324, (2006).

47. C. Yan, P. Adderley et al., Nucl. Instr. Meth. A365, 46, (1995).

48. R. Wojcik and C. Yan, Nucl. Inst. Meth. A484, 690, (2002).

49. M.Q. Tran et al., Phys. Lett. B 445, 20, (1998).

50. T. Motoba et al., Prog. Theor. Phys. Suppl. 117, 123, (1994).

51. C.E. Hyde-Wight et al., Electron scattering at 0 degree - A photon tagging technique.

52. C.E. Hyde-Wright, W. Bertozzi, J.M. Finn, In Proc. 1985 CEBAF Summer Workshop, p.1, Newport News, VA, (1985).

53. Yung-Su Tsai, Rev. Mod. Phys. 46-815, (1974).

54. Y. Okayasu, Ph.D. thesis, JLAB-PHY-08-889, September (2009) (http://www1.jlab.org/U1/Publications/documents/Yuichi-dthesis_letter.pdf).

55. L. Tang, HKS Target Ladder Exchange, (Hall C How To, collaboration document).

56. Cortesy of E01-011 (HKS) internal documents., (2001)-(2005).

57. H. A. Enge, Nucl. Instr. Meth. 28, 119, (1964).

58. J. E. Spencer and H. A. Enge, Nucl. Instr. Meth. 49, 181, (1967).

59. Y. Okayasu, Design of the HKS water Cherenkov counter, (E01-011 How-To manual, unpublished), May 10, (2005). 
60. J. Reinhold et al., HKS Technical Note, (HKS collaboration internal document).

61. F.J. Barbosa et al., The Jefferson Lab High Resolution TDC Module, 0-7803-76366/03, IEEE, (2003).

62. O. Hashimoto, J. Reinhold, L. Tang, S. Nakamura, The present status of JLab E01011 experimentl, JLab, (2003).

63. CODA-CEBAF, On-line Data Aquisition User's Manual, JLab, (1995).

64. V. Gyurjyan et al. JLab data acquisition run control system, Proc. CHEP04, 29 Sep., Interlaken, Switzerland, (2004).

65. P. A. Cherenkov, Comptes Rendus Acad. Sciences USSR, 2, 451, (1934).

66. R. Fernow, Introduction to Experimental Particle Physics, Cambridge University Press, New York, (1986).

67. T. Ypsilantis and J. Seguinot, Nucl. Instrum. Methods A343, 30, (1994).

68. M. Carl, M.S. thesis, FIU, (2003).

69. I. van der Werfa, F. Palmisanoa, et al., Chemical Analyses of Silicon Aerogel Samples, 0804.3455, arxiv.org.

70. V. Popov, Nucl. Instr. Meth. in Physics Research Section A, Volume 505, Issue 1-2, p. $316,(2003)$.

71. M. Benot et al., Nucl. Instr. Meth. 154, 253, (1978).

72. M. Carl et al. Nucl. Instr. Meth. A527, 301, (2004).

73. S. Wood, The CEBAF Test Package, Newport News, VA, 1995, Internal document.

74. S. Penner, Rev. Sci. Instr. 32, 150, (1961).

75. L. Yuan, L. Tang, Data analysis report, internal colaboration document (not published).

76. L. Yuan, L. Tang, Optical calibration for Jefferson Lab HKS spectrometer, arxiv:nucl-ex/0511011v1, Nov. 4, (2005).

77. D.J. Millener, private communications.

78. M. Sotona, S. Frullani, Prog. Theor. Phys. Suppl. 117, 151, (1994). 
79. T. Mizutani et al., Phys. Rev. C 58, 75, (1998).

80. M. Iodice et al., High Resolution Spectroscopy of 12B-Lambda by Electroproduction, Phys. Rev. Lett. 99, 052501, (2007).

81. L. Yuan, private communications.

82. M. Youn, Hypernuclear spectroscopy for 12B-Lambda through (K-stop,pi0) reaction using NMS, proceedings of APCTP Workshop SNP99, World Scientific, p.75 (2000).

83. H.G. Juengst et al., Energy Calibration of the CsI Crystals of the Neutral Meson Spectrometer (NMS) using Monoenergetic $\mathrm{p}^{\circ}$ from the Decay of Stopped K, Proc. Conf. on Calorimetry in High Energy Physics (CALOR 99).

84. L. Yuan et al. Phys. Rev. C, 73, 044607, (2006).

85. M. Agnello et al., Phys. Lett. B 622, 35-44, (2005).

86. K. Itonaga et al., Phys. Rev. C, Vol. 49, 2, (1994).

87. E. Hiyama et al., Phys. Rev. C 53, 5, 2075, (1996).

88. E. Hiyama, Phys. Rev. C 80, 054321, (2009).

89. E. Hiyama et al., Phys. Rev. C 65, 011301(R), (2002).

90. M. Juric et al., Nucl. Phys. B52, 1, (1973).

91. O. Hashimoto et al. Nucl. Phys. A 639, 93c, (1998).

92. M. Sotona, private communications.

93. H. Tamura et al. Phys. Lett. B84, 5963, (2000).

94. J.C. David, C. Fayard, G.-H. Lamot, B. Saghai, Phys. Rev. C 53, (1996).

95. T. Mizutani et al., Phys. Rev. C 58, 75, (1998).

96. T. Mart and C. Bennhold, Phys. Rev. C 61, 012201(R), (1999).

97. R. A. Adelseck and B. Saghai, Phys. Rev. C 42, 108, (1990).

98. W.A. Williams et al. Phys. Rev. C, 46, 1617, (1992).

99. P. Bydzovsky et al., Photo- and electro-production of medium mass Lambdahypernuclei (arXiv:0706.3836v1 [nucl-th]), 26 Jun. (2007). 
100. T. Motoba et al., Phys. Rev. C 38, \#3, (1988).

101. L. Tang, Target ladder howto manuals, internal colaboration document, (2005). 


\section{APPENDIX A}

This appendix section includes the data description for ${ }^{12} \mathrm{C},{ }^{7} \mathrm{Li}$, and ${ }^{28} \mathrm{Si}$ targets presented in Tables 1A, 2A, and 3A, respectively. The likelihood PID routine used primarily information from the first table.

Table 1.A Data summary for ${ }^{12} \mathrm{C}$ target $\left({ }_{\Lambda}^{12} \mathrm{~B}\right.$ hypernucleus).

\begin{tabular}{|c|c|c|c|c|c|c|}
\hline Part \# & Runs range & $\begin{array}{l}\# \text { of } \\
\text { runs }\end{array}$ & $\mathrm{I},(\mu \mathrm{A})$ & Trigger conditions & $\begin{array}{c}\text { True } \\
\text { cointime }\end{array}$ & Beta fractions $\left(\beta_{\mathrm{p}}+\beta_{K}+\beta_{\pi}=1\right)$ \\
\hline 1 & $56165-56235$ & 35 & 17 & $\begin{array}{c}\text { 1X\&2X\&WC\& } \overline{A C} \\
\text { ungrouped }\end{array}$ & 2.82 & $\beta_{\mathrm{p}}=0.408 ; \beta_{K}=0.092 ; \beta_{\pi}=0.501$ \\
\hline 2 & $56265-56278$ & 10 & $\begin{array}{c}10,17 \\
30\end{array}$ & same as above & 1.73 & $\beta_{\mathrm{p}}=0.432 ; \beta_{K}=0.062 ; \beta_{\pi}=0.507$ \\
\hline 3 & $56355-56379$ & 18 & $\begin{array}{l}30,20 \\
18\end{array}$ & $\begin{array}{c}1 \mathrm{X} \& 2 \mathrm{X} \& \mathrm{WC} \& \overline{\mathrm{AC}} \\
\text { grouped }(6 \mathrm{w} / \text { overlap) }\end{array}$ & 1.75 & $\beta_{\mathrm{p}}=0.512 ; \beta_{K}=0.069 ; \beta_{\pi}=0.420$ \\
\hline 4 & $56382-56405$ & 19 & 18,30 & same as above & 3.05 & $\beta_{\mathrm{p}}=0.451 ; \beta_{K}=0.072 ; \beta_{\pi}=0.477$ \\
\hline 5 & $56406-56431$ & 12 & 30 & same as above & 3.04 & $\beta_{\mathrm{p}}=0.416 ; \beta_{K}=0.071 ; \beta_{\pi}=0.512$ \\
\hline 6 & $56467-56473$ & 5 & $\approx 20$ & same as above & -0.02 & $\beta_{\mathrm{p}}=0.392 ; \beta_{K}=0.125 ; \beta_{\pi}=0.483$ \\
\hline 7 & $56528-56569$ & 32 & 28 & same as above & 38.01 & $\beta_{\mathrm{p}}=0.403 ; \beta_{K}=0.071 ; \beta_{\pi}=0.526$ \\
\hline 8 & $56575-56628$ & 41 & 28 & same as above & 37.89 & $\beta_{\mathrm{p}}=0.371 ; \beta_{K}=0.072 ; \beta_{\pi}=0.557$ \\
\hline 9 & $56665-57200$ & 366 & $\begin{array}{l}28,24 \\
18,12\end{array}$ & same as above & 37.89 & $\beta_{\mathrm{p}}=0.341 ; \beta_{K}=0.062 ; \beta_{\pi}=0.597$ \\
\hline 10 & $57547-57645$ & 80 & 24 & same as above & 37.27 & $\beta_{\mathrm{p}}=0.352 ; \beta_{K}=0.048 ; \beta_{\pi}=0.600$ \\
\hline 11 & $57683-57727$ & 38 & $\begin{array}{c}24,18 \\
12\end{array}$ & same as above & 37.23 & $\beta_{\mathrm{p}}=0.800 ; \beta_{K}=0.042 ; \beta_{\pi}=0.158$ \\
\hline 12 & $57729-57734$ & 5 & 24 & same as above & 36.92 & $\beta_{\mathrm{p}}=0.861 ; \beta_{K}=0.029 ; \beta_{\pi}=0.110$ \\
\hline 13 & $57736-57780$ & 31 & 20,24 & same as above & 36.67 & $\beta_{\mathrm{p}}=0.811 ; \beta_{K}=0.031 ; \beta_{\pi}=0.158$ \\
\hline 14 & $57781-57830$ & 37 & 24 & same as above & 36.97 & $\beta_{\mathrm{p}}=0.821 ; \beta_{K}=0.042 ; \beta_{\pi}=0.137$ \\
\hline 15 & $58401-58408$ & 8 & 24 & same as above & 36.82 & $\beta_{\mathrm{p}}=0.816 ; \beta_{K}=0.040 ; \beta_{\pi}=0.144$ \\
\hline 16 & $58410-58669$ & 122 & 24,22 & same as above & 37.01 & $\beta_{\mathrm{p}}=0.521 ; \beta_{K}=0.060 ; \beta_{\pi}=0.419$ \\
\hline 17 & $58670-59510$ & 83 & 24,26 & same as above & 37.12 & $\beta_{\mathrm{p}}=0.678 ; \beta_{K}=0.062 ; \beta_{\pi}=0.261$ \\
\hline 18 & $59872-59882$ & 7 & 13 & same as above & 26.75 & $\beta_{\mathrm{p}}=0.538 ; \beta_{K}=0.072 ; \beta_{\pi}=0.390$ \\
\hline
\end{tabular}




$1959954-60561 \quad 38 \quad 26,13 \quad$ same as above $\quad-2.67 \quad \beta_{\mathrm{p}}=0.563 ; \beta_{K}=0.056 ; \beta_{\pi}=0.381$

Table 2.A Data summary for ${ }^{7} \mathrm{Li}$ target $\left({ }_{\Lambda}^{7} \mathrm{He}\right.$ hypernucleus).

\begin{tabular}{cccccc}
\hline Part \# & Runs range & $\begin{array}{c}\# \text { of } \\
\text { runs }\end{array}$ & $\mathrm{I},(\mu \mathrm{A})$ & Trigger conditions & $\begin{array}{c}\text { True } \\
\text { cointime }\end{array}$ \\
\hline 1 & $60236-60461$ & 106 & $26,30,27$ & 1X\&2X\&WC\& $\overline{\mathrm{AC}}$ grouped & -2.62 \\
\hline 2 & $60462-61067$ & 105 & 27,30 & same as above & -2.62 \\
\hline
\end{tabular}

Table 3.A Data summary for ${ }^{28} \mathrm{Si}$ target $\left({ }_{\Lambda}^{28} \mathrm{Al}\right.$ hypernucleus $)$.

\begin{tabular}{cccccc}
\hline Part \# & Runs range & $\begin{array}{c}\# \text { of } \\
\text { runs }\end{array}$ & $\mathrm{I},(\mu \mathrm{A})$ & Trigger conditions & $\begin{array}{c}\text { True } \\
\text { cointime }\end{array}$ \\
\hline 1 & $57248-58244$ & 325 & $12,13,14,20$ & 1X\&2X\&WC\& $\overline{\mathrm{AC}}$ grouped & 39.43 \\
\hline 2 & $58245-58394$ & 112 & 12,13 & same as above & 39.45 \\
\hline 3 & $58720-59098$ & 297 & 13,15 & same as above & 39.73 \\
\hline 4 & $59100-59638$ & 312 & 13 & same as above & 39.74 \\
\hline 5 & $59640-60756$ & 318 & $7,10,13,18$ & same as above & -0.049 \\
\hline
\end{tabular}

The likelihood routine is embedded into physics.f file that is a part of the standard Hall $\mathrm{C}$ analyzer. The routine employs six parameter files that contain parameters for pdf functions and a set of flags. The beta fractions described in detail in the Thesis text are written in 19 files. These 19 files originally were created to parameterize the ${ }^{12} \mathrm{C}$ data. Since the data on ${ }^{12} \mathrm{C}$ covers almost all of the data production time, the beta fraction parameter files are valid for using together with other targets. The beta fraction parameter files are called "bfrac.param.num", where "num" corresponds to the number of the first run in the data series. The table below (Table 4.A) shows this numbering together with the six likelihood parameter files that are used to describe all targets. 
Table 4.A List of parameter files used in likelihood PID routine.

\begin{tabular}{|c|c|c|}
\hline bfrac.param.--> & $56165 \quad 56265 \quad 56355$ & $\begin{array}{llll}56382 & 56406 & 56467 & 56528 \\
\end{array}$ \\
\hline & hprob.param.55959 & hprob.param.56382 \\
\hline bfrac.param.-> & $57547 \quad 57683$ & $\begin{array}{llll}57729 & 57736 & 57781 & 58401\end{array}$ \\
\hline & hprob.param.56648 & hprob.param.56648 \\
\hline bfrac.param.-> & $\begin{array}{lll}58410 & 58670 & 59872\end{array}$ & rest of data \\
\hline
\end{tabular}

The flags inside of parameter files are used by the likelihood routine to select both the pdf functions types and the way the final likelihood values are formed. The information about the flags is summarized in Table 5.A.

Table 5.A List of the flags used in likelihood PID routine.

\begin{tabular}{|c|c|c|}
\hline \multicolumn{2}{|c|}{ Beta method switch } & Switch used = "1" \\
\hline “0” & \multicolumn{2}{|l|}{$\operatorname{Gaussian}(p, K, \pi)$} \\
\hline "1" & \multicolumn{2}{|c|}{ Left Voigt $(p)$, Central Voigt $(K)$, Right Voigt $(\pi)$} \\
\hline "2" & \multicolumn{2}{|c|}{ Left Voigt $(p)$, Gaussian $(K)$, Right Voigt $(\pi)$} \\
\hline “3” & \multicolumn{2}{|c|}{4 momentum dependent Left Voigts $(p)$, Central Voigt $(K)$, Right Voigt $(\pi)$} \\
\hline \multicolumn{2}{|c|}{ Aerogel Cherenkov method switch } & Switch used = "0" \\
\hline "0" & \multicolumn{2}{|c|}{ Left Voigt $(p, K)$, Right Voigt $(\pi)$} \\
\hline "1" & \multicolumn{2}{|c|}{ Map read-out from file (pdf map) } \\
\hline "2" & \multicolumn{2}{|c|}{ Gaussian $(p, K)$, Map read-out from file $(\pi)$} \\
\hline \multicolumn{2}{|c|}{ Water Cherenkov method switch } & Switch used = “3” \\
\hline “0” & \multicolumn{2}{|l|}{ Layered WC pdfs } \\
\hline "1" & \multicolumn{2}{|l|}{ Segmented WC pdfs } \\
\hline "2" & \multicolumn{2}{|c|}{ Right Voigt $(p)$, Poisson $(K, \pi)$} \\
\hline "3" & \multicolumn{2}{|c|}{ six momentum dependent Right Voigts $(p, \pi)$, Poisson $(K)$} \\
\hline \multicolumn{2}{|c|}{ Likelihood method switch } & Switch used = "1" \\
\hline "0" & \multicolumn{2}{|l|}{ Without beta fractions } \\
\hline$" 1 "$ & \multicolumn{2}{|l|}{ With beta fractions } \\
\hline " $2 "$ & \multicolumn{2}{|l|}{ With inverted beta fractions } \\
\hline
\end{tabular}


The coin ntuples used in the analysis contain 109 variables. These ntuples included the events, which were counted as coincidences between the two arms. The parameterization for the likelihood routine was done using specifically created for that purpose ntuples, which only contained the events counted in HKS arm. These ntuples used the external flags to switch on and off different variables. The flags used for this purpose are listed in the Table 6.A. The values of the flags were varied depending on the parameterization task needed.

Table 6.A Summary of flags used for creating ntuple for likelihood parameterization.

\begin{tabular}{lcl}
\hline Switch Variable & $\begin{array}{c}\text { Switch position } \\
\text { ("0"- OFF, “1" - ON) }\end{array}$ & Purpose \\
\hline ac1 & 0 & AC layer 1 ADC\&TDC \\
\hline ac2 & 0 & AC layer 2 ADC\&TDC \\
\hline AC3 & 0 & AC layer 3 ADC\&TDC \\
\hline unb & 0 & Unbiased aerogel ADC \\
\hline aseg & 1 & Segmented aerogel ADC \\
\hline wseg & 0 & Segmented water ADC \\
\hline w1 & 0 & WC layer 1 ADC\&TDC \\
\hline w2 & 0 & WC layer 2 ADC\&TDC \\
\hline x1 & 0 & HKS X1 hodo ADC\&TDC \\
\hline x2 & 0 & HKS X2 hodo ADC\&TDC \\
\hline atul & 0 & Aerogel TUL TDC vars // det\#3 \\
\hline wtul & 0 & WC TUL TDC vars // det\#4 \\
\hline xtul1 & 0 & X1 TUL TDC vars // det\#1 \\
\hline xtul2 & 0 & X2 TUL TDC vars // det\#2 \\
\hline group & 0 & Grouping flags // Trig. group vars \\
\hline phys & 1 & Physics Variables \\
\hline pdf & 0 & pdf functions \\
\hline lh & 1 & likelihoods \\
\hline lhcon & 0 & conditional likelihoods \\
\hline Physevn & 1 & $1:$ HKS events 2: COIN events \\
\hline
\end{tabular}




\section{APPENDIX B}

This appendix describes cut conditions used for particle identification. The PID has been performed by two methods: standard (hard cuts) and likelihood. The conditions used in standard hard cuts approach are shown in Table 1.B and the cuts applied in likelihood routine are presented in Table 2.B.

Table 1.B Summary of the hard cuts used in standard approach to PID.

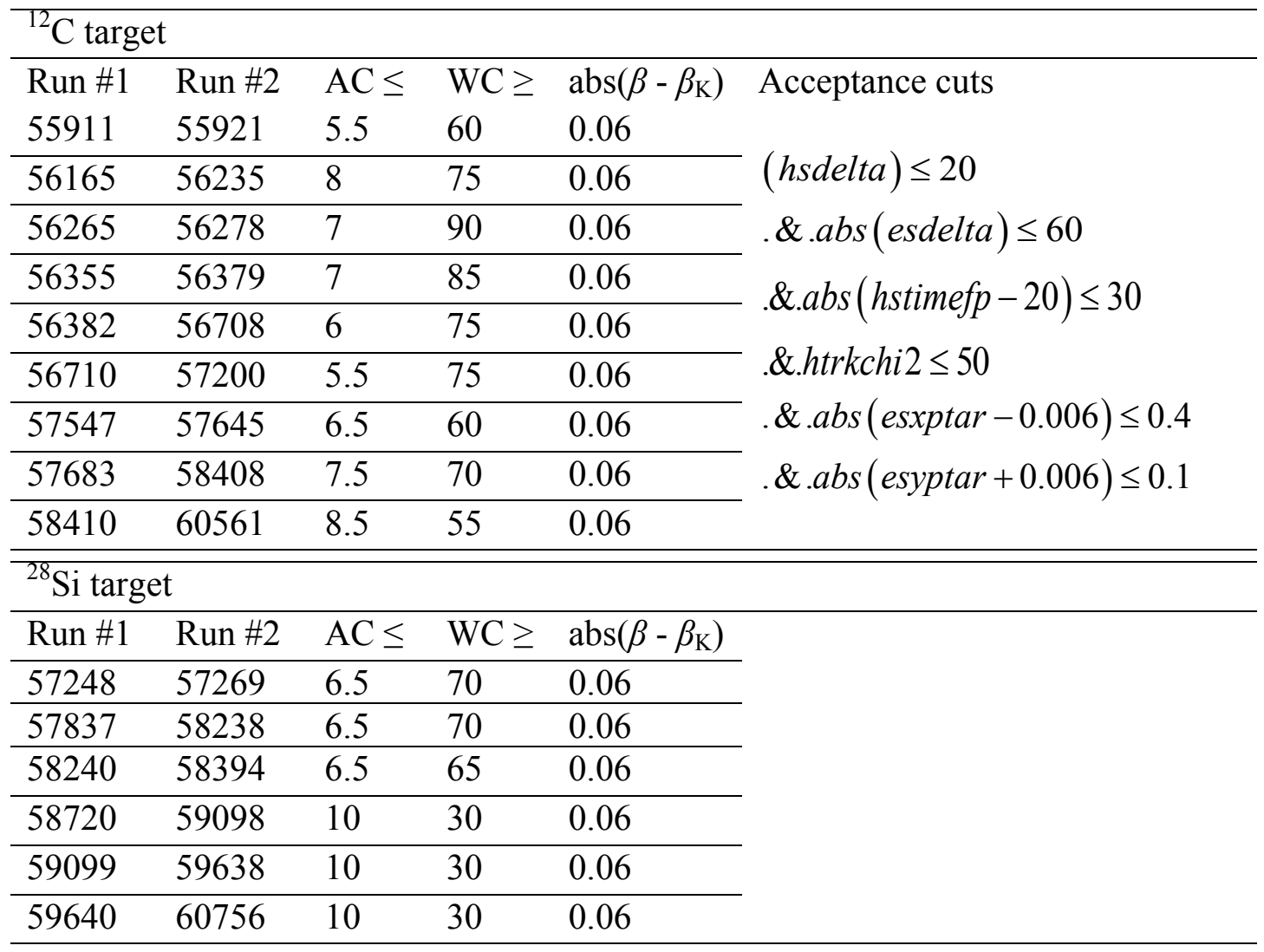


Table 2.B Cuts used in likelihood PID analysis.

\begin{tabular}{ll}
\hline Likelihood cuts & Acceptance cuts \\
\hline & $($ hsdelta $) \leq 20 . \& . a b s($ esdelta $) \leq 60$ \\
& .\&.abs $($ hstimefp -20$) \leq 30 . \&$.htrkchi $\leq 50$ \\
$\left(L_{K}>L_{p}\right)$.and. $\left(L_{K}>L_{\pi}\right)$ & $. \& . a b s($ esxptar -0.006$) \leq 0.4$ \\
& .\&.abs $($ esyptar +0.006$) \leq 0.1$ \\
\hline
\end{tabular}


VITA

\section{PAVLO BATURIN}

1999

$1999-2001$

$2001-2003$

2003

2003 - present
Born, Poltava, UKRAINE

M.S., Physics (diploma with award)

Sumy State University

Department of Physics and Technology

Sumy, UKRAINE

Research Scientist / Engineer

Institute of Applied Physics

Department of Theoretical Physics

Sumy, UKRAINE

Research / Teaching Assistant

Case Western Reserve University

Physics Department

Cleveland, OH, USA

M.S., Physics

Case Western Reserve University

Physics Department

Cleveland, OH, USA

Research / Teaching Assistant

Florida International University

Physics Department

Miami, FL, USA

\section{PUBLICATIONS AND PRESENTATIONS}

- V.V. Kulish, A.G. Kayluk, P. Baturin, The single particle model of maser on cyclotron resonance, Ukrainian Physical Journal, V.43, \#4, P. 398-403, 1998.

- R.I. Kholodov, P. Baturin, Polarization effects in synchrotron radiation in ultraquantum approximation, Ukrainian Journal of Physics, V.46, \#5-6, P. 621-626, 2001.

- P. Baturin, The Challenges of QCD and the Opportunities of the $12 \mathrm{GeV}$ Upgrade, Users Workshop \& Annual Meeting, JLab, Newport News, VA, June 20-22, 2005.

- Magnetic Field Test (Results), HKS Collaboration meeting, Feb, 2005, JLab, Newport News, VA / (presentation)

- Particle Identification with the Likelihood Method, E01-011 (HKS) Collaboration Meeting at FIU Dec. 09, 2006, Miami, FL / (presentation)

- Likelihood PID Update, E01-011 (HKS) Collaboration Meeting, Mar 15, 2007, JLab, Newport News, VA / (presentation) 
- L. Tang, L. Yuan et al., E01-011 Coll., The HKS experiment on lambda-hypernuclear spectroscopy via electroproduction at JLab, Nucl. Phys. A790, 679-682, 2007.

- M. Iodice et al., JLab Hall A Coll., High Resolution Spectroscopy of B-12 (Lambda) by Electroproduction, Phys. Rev. Lett., 99, 052501, 5 pp, 2007.

- O. Hashimoto et al., E01-011 Coll., Hypernuclear spectroscopy program at JLab Hall$C$, Nucl. Phys. A804: 125-138, 2008.

- Y. Song et al., E02-017 Coll., Kaon, pion and proton associated fission of Bi nuclei with electromagnetic probes, Preprint (PRC-Rapid Communication 9/20/2008).

- P. Baturin, Novel Likelihood PID Method for Hypernuclear Spectroscopy, Southeastern Section of APS (SES08), Oct. 31 2008, Raleigh, NC / (presentation)

- F. Cusanno et al., Hall A Coll., High Resolution Spectroscopy of N-16 Lambda by electroproduction, Phys. Rev. Lett., 103(20), 2009.

- Likelihood PID (Test on C12 target), HKS-HES Collaboration Meeting, May 08, 2009, JLab, Newport News, VA / (presentation)

- D. Kawama et al., The Third Generation (e, $\left.\mathrm{e}^{\prime} K^{+}\right) \Lambda$ Hypernuclear Spectroscopy at JLab, Nuclear Physics Review, 26 (suppl): 88-93, July 2009.

- P. Baturin, Application of Likelihood PID Method in Hypernuclear Experiment E01011, Joint DNP \& JPS, Oct 15, 2009, Hawaii's Big Island, HI / (presentation).

- P. Baturin, Spectroscopy of Lambda Hypernuclei in the (e, $\left.\mathrm{e}^{\prime} K^{+}\right)$Reaction at Jefferson Lab, XIII International Conf. on Hadron Spectroscopy, Tallahassee, FL, Nov. 30, 2009 / (presentation).

- P. Baturin, Spectroscopy of Lambda Hypernuclei in the (e, $\left.\mathrm{e}^{\prime} K^{+}\right)$Reaction at Jefferson $L a b$, Proc. of XIII Int. Conf. on Hadron Spectroscopy, submitted to AIP, 01/2010.

- O. Hashimoto et al., Hypernuclear Spectroscopy at JLab Hall C, Nuclear Physics A, 835, pp. 121-128, 2010

- P. Baturin, High resolution Lambda hypernuclei spectroscopy with $\left(\mathrm{e}, \mathrm{e}^{\prime} K^{+}\right)$reaction, High Energy Nuclear Physics and QCD Workshop, FIU, Miami, FL, 2/3/10-2/6/10 / (presentation).

- P. Baturin, High resolution Lambda spectroscopy in Jefferson Laboratory via (e, $\left.\mathrm{e}^{\prime} K^{+}\right)$ reaction, April APS meeting, Washington D.C., Feb. 16, 2010 / (poster).

- P. Baturin, High resolution $\Lambda$ hypernuclear spectroscopy at JLab Hall C, Annual User Group Meeting, Newport News, VA, June 7, 2010 / (poster).

- High Resolution Hypernuclear Spectroscopy at Jefferson Laboratory, Carnegie Mellon University, July 7, 2010, Pittsburgh, PA / (invited talk).

- P. Baturin, Spectroscopy of Electroproduced Light to Medium Mass Lambda Hypernuclei, Gordon Research Conference on Photonuclear Reactions, 08/01/1008/06/10, Tilton, $\mathrm{NH} /$ (poster). 